

Extension and Enhancement Methods for Setting Data Quality Objectives

Final Report

D. Goodman

March 2000

Work Performed Under Contract No. DE-FC07-94ID13317

For

U.S. Department of Energy Assistant Secretary for Environmental Management Washington, DC

By

Montana State University

Bozeman, MT 


\section{DISCLAIMER}

This report was prepared as an account of work sponsored by an agency of the United States Government. Neither the United States Government nor any agency thereof, nor any of their employees, make any warranty, express or implied, or assumes any legal liability or responsibility for the accuracy, completeness, or usefulness of any information, apparatus, product, or process disclosed, or represents that its use would not infringe privately owned rights. Reference herein to any specific commercial product, process, or service by trade name, trademark, manufacturer, or otherwise does not necessarily constitute or imply its endorsement, recommendation, or favoring by the United States Government or any agency thereof. The views and opinions of authors expressed herein do not necessarily state or reflect those of the United States Government or any agency thereof. 


\section{DISCLAIMER}

\section{Portions of this document may be illegible in electronic image products. Images are produced from the best available original document.}




\title{
EXTENSION AND ENHANCEMENT METHODS FOR SETTING DATA QUALITY OBJECTIVES
}

\author{
FINAL REPORT
}

D. Goodman

March 2000

Work Performed Under Contract No. DE-FC07-94D13317

Prepared for the U.S. Department of Energy

Assistant Secretary for Environmental Management

Washington, DC

\author{
Prepared by \\ Montana State University \\ Bozeman, MT
}


FINAL REPORT OF THE

\title{
MSU COOPERATIVE AGREEMENT DE-FC07-94ID13317 \\ "EXTENSION AND ENHANCEMNENT METHODS FOR \\ SETTING DATA QUALITY OBJECTIVES"
}

\author{
by \\ Dr. Daniel Goodman
}

March 3, 2000 


\section{FINAL REPORT OF THE \\ MSU COOPERATIVE AGREEMENT DE-FC07-94ID13317 \\ "EXTENSION AND ENHANCEMNENT METHODS FOR SETTING DATA QUALITY OBJECTIVES"}

\section{INTRODUCTION}

The objective of this project was to develop statistical tools for the application of decision theory and operations research methods, including cost-benefit analysis, to the DQO process. Our efforts combined

(1) methods developed to produce validated peer reviewed statistical methodology specifically designed for carrying out technical optimization in the $\mathrm{DQO}$ process,

(2) algorithm development to make the statistical tools accessible to a wider audience,

(3) algorithm development to make environmental data accessible for decision making purposes, and

(4) direct involvement in pilot projects to demonstrate the implementation of these methods for environmental decision processes at real sites.

We have made substantial contributions in all four areas during the course of the project.

\section{ACCOMPLISHMENTS}

\section{Methods Development}

Our focus in methods development has been on two topics, spatial statistics (geostatistics) and statistics for merging data sets (meta-analysis). Both are extremely important to the process of site remediation.

\section{a. Spatial statistics}

Our primary concern in spatial statistics was with the objectivity and reproducibility of maps of contamination, made by the use of a technique called "kriging", for interpolating between and extrapolating from sample data from restricted locations.

We convened a special team to carry out an objective comparison of commercially available kriging software. We documented that the available software, when left to their respective "default" settings, deliver quite different answers for the estimates of contamination (an interpolated map), when given real data from a contaminated site. 
This raises the potential for inconsistencies if different software is used; and it motivates the question: "which estimate is the right one to use?"

We diagnosed the differences between estimates delivered by different kriging software as owing primarily to different search rules for kriging points, and only secondarily to differences in variogram estimation. Since the reason for search rules has more to do with computational convenience than statistical theory, these differences are unnecessary, and we found effective ways to bypass it.

The available packages were generally deficient in quantification of uncertainty for quantities of actual interest, such as inventories over a volume. Calculations of uncertainty are very demanding of computer speed and memory, especially in 3applications. For these reasons we developed our own programs, with emphasis on computational efficiency, for uncertainty quantification.

Major interim reports on our methods development work for kriging included: the Report on Effects of Variance Heterogeneity in Kriging; the report on a Standardizable Approach to Kriging; the report on Implementation Algorithms for Composite Likelihood Estimation of the Variogram for Spatial Interpolation; the report on Detrending Approaches in Kriging; and the report on Frequency Domain Conditional Simulation.

In this final report, we document, in Appendix 1, a comprehensive package of kriging methodology that we applied successfully in a pilot study at the Hanford site.

b. Objective Bayesian methodology for risk assessment and for merging data sets

We developed a mathematical approach to Bayesian statistical inference that removes the need for a subjective prior distribution and that removès the need for a subjective definition of probability. Results were reported in: the paper on Objective Bayesian Analysis presented at the symposium on Statistical Inference at the ESA annual meeting in Baltimore; a chapter on Site Cleanup, Cost and Risk Control published in the Wiley Encyclopedia of Environmental Analysis and Remediation; a new manuscript on Risk Analysis for Population Extinction Risk; and a paper on Extrapolation in Risk Assessment presented at the EPA NHEERL Annual Risk Symposium.

These methods have been applied successfully in estimating mortality rates for endangered species populations.

\section{Statistical Algorithm Development}

We completed implementation of a web version of the sampling design program, SAMPLAN. This in now up on our web page: www.esg.montana.edu. This version has all the features of the PC-based version, with of course better spreadsheets and scrolling. 
We completed a report with a mathematical proof of a new recursion algorithm for obtaining a common probability distribution that combines information from several studies. This will simplify the estimation of Bayesian prior distributions for metaanalyses.

We have adapted use of the visualization package, VIS-5d, developed with NASA funding for meteorological applications, to display concentration and uncertainty 3-D "maps" for computer estimates of contamination plumes. This allows detailed zoom, slice-and-dice, rotation, contouring and probing, which greatly facilitates communication about these complicated 3-D objects. This visualization technology was applied successfully in our pilot study at the Hanford site, as documented in Appendix 2.

\section{Data Access Algorithm Development}

We developed a map-interface system, with national coverage, for linking geo-referenced information with respect to location, aggregating by aerial distance, political boundaries, watershed boundaries, or ecoregions. This system is available to the public on the web (www.esg.montana.edu), where it receives heavy usage.

We have successfully applied this system as an interface for an environmental data base of the US Fish and Wildlife Service. The map-interface.system is documented here in Appendix 3.

\section{Pilot Studies}

We provided technical support in the DQO for vadose zone characterization to support TWRS decisions for retrieval of contents of the single shell tanks at Hanford. We used some of our spatial statistics techniques for analysis of sampling data from dry wells at SX-Tank Farm; and we used our adaptation of the VIS 5-d visualization package to display the results for discussions during the DQO process. This has been especially effective for exploration of scenarios in the decisions about location and orientation (slant drilling is now under consideration) of new test wells. This work culminated in estimation of the inventory of contamination under the SX-Farm, which is reported in Appendix 2. 


\section{BUDGET}

The following table shows the money spent for this year of the project:

\begin{tabular}{|l|r|}
\hline & Total this Year \\
\hline SALARIES & $73,459.06$ \\
\hline BENEFITS & $13,586.58$ \\
\hline REP \& MNT & 202.35 \\
\hline TRAVEL & $2,419.77$ \\
\hline COMMUN & 749.63 \\
\hline CONT SERV & 840.56 \\
\hline SUPPLIES & $3,131.69$ \\
\hline SUB CONT & 46.07 \\
\hline RENT & 0.00 \\
\hline Minor Equipment & $3,799.00$ \\
\hline EQUIPMENT & $15,832.18$ \\
\hline AWARDS & $7,618.10$ \\
\hline DIRECT COSTS & $121,684.99$ \\
\hline IDCs (39\%) & $38,311.54$ \\
\hline TOTAL & $159,996.53$ \\
\hline
\end{tabular}

\section{Attachments}

Appendix 1 - Documentation of Geostatistical Approach for the Analysis of SXFarm Vadose Zone Gamma Data

Appendix 2-Estimation of SX-Farm Vadose Zone Cs-137 Inventories from Geostatistical Analysis of Drywell and Soil Core Data

Appendix 3-Graphical Locator 
Appendix 1 


\title{
DOCUMENTATION OF GEOSTATISTICAL APPROACH FOR THE ANALYSIS OF SX-FARM VADOSE ZONE GAMMA DATA *
}

\author{
Daniel Goodman \\ Environmental Statistics Group \\ Biology Department \\ Montana State University \\ Bozeman, MT 59717
}

September 27, 1999

\section{Contents}

1 INTRODUCTION ................... 2

2 STANDARDIZATION IN GEOSTATISTICAL PRACTICE $\ldots \ldots \ldots$

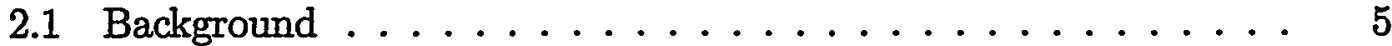

2.2 EPA Comparison Study $\ldots \ldots \ldots \ldots \ldots \ldots \ldots \ldots$

2.3 MSU Comparison Study . . . . . . . . . . . . . . . 7

2.4 Kriging Choices and Prediction Uncertainty $\ldots \ldots \ldots \ldots \ldots .9 .9$

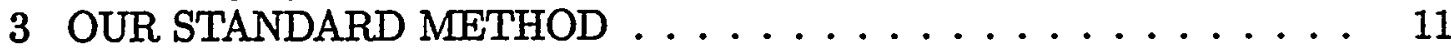

3.1 The Second-Order Stationary Gaussian Process . . . . . . . 11

3.2 The Exponential Variogram Model . . . . . . . . . . . 11



4 APPLICATION TO THE SX-FARM DATA SET $\ldots \ldots \ldots \ldots .14$

5 MATHEMATICAL REPRESENTATION . . . . . . . . . . . 19

5.1 Notation . . . . . . . . . . . . . . 19

* This is a report to Mactec Inc., under agreement 3751.119.376 with Montana State University. Portions of the work reported here were supported by DOE cooperative agreement DE-FC07-94ID13317 to Montana State University, Daniel Goodman, Principal Investigator. 
5.2 Model of the Underlying Process $\ldots \ldots \ldots \ldots$

5.3 Spatial Structure of the Underlying Process . . . . . . . . . 19

5.4 Relating Observations to the Underlying Process . . . . . . . . 20

5.5 Spatial Structure of the Observations . . . . . . . . . . . 20

5.6 Joint Spatial Structure of Observations and the Underlying Process 20

5.7 Joint Distribution of Observations and the Underlying Process . . 20

5.8 Conditional Distribution of the Process, Given the Observations . 21

5.9 Likelihood Function . . . . . . . . . . . . . . . . 21

5.10 Computational Demands and Computational Strategy . . . . . . 21

6 RESULTS AND FUTURE DIRECTIONS . . . . . . . . . . . . 22

6.1 Visual Inspection and Interpretation of Results . . . . . . . . 23

6.2 Calibrating Plume Estimates for Data Sets Without Laterals . . . 25

6.3 Correlating Plume Inventory Estimates and Leak Inventory . . . . 25

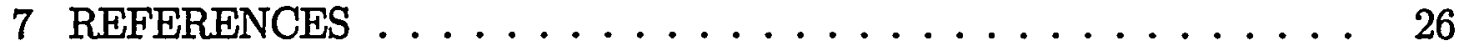

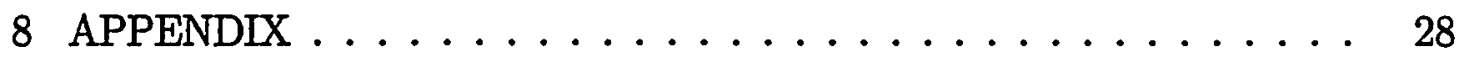

\section{INTRODUCTION}

The SX tank farm comprises 15 tanks, some of which have been involved in significant leak events. For many years, the tank farm was monitored by gross gamma logging in 99 vertical drywells, most of which were located near the perimeters of the tanks. More recently, most of these vertical drywells were re-measured by spectral gamma logging. In addition to the vertical drywells, gross gamma logging data was available from lateral drywells under eight of the tanks. (There were lateral drywells under two more of the tanks, but the data from these were not available.) Further, a modest number of soil samples were analyzed from soil cores taken near two of the tanks, in positions closer to the tank wall than the perimeter drywells. Figure 1 shows the locations of all wells with data.

The objective in conducting geostatistics on the gamma data from the SX tank farm was to compute statistically defensible reconstructions of the contamination plumes, with quantified uncertainty (confidence) bounds. These reconstructions could later serve for calculation of plume inventories, that might be compared against calculations of leak inventories, in order to better understand the pattern, extent, and possible dynamics of the vadose zone contamination. The spatial pattern of the computed uncertainty would also be helpful in decisions about where additional future measurements and sampling might prove most informative. 


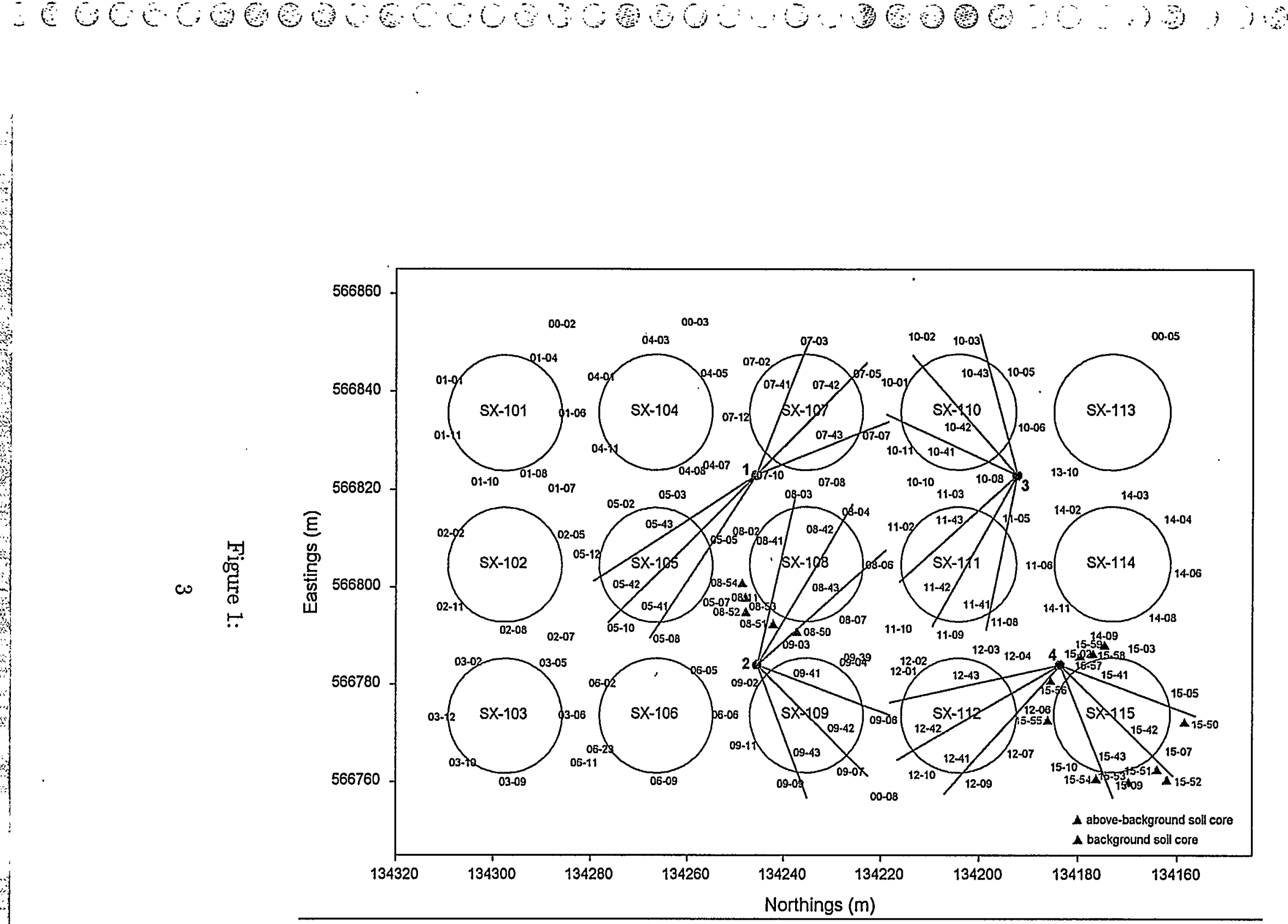


Additionally, the availability of lateral data in SX-farm presents an opportunity for calibrating the ability of perimeter vertical drywell monitoring to detect and quantify leak plumes. Being located directly under the tanks, the laterals are better positioned to intercept leaks, especially when they are too small to have spread much horizontally. But many of the other Hanford tank farms don't have lateral well data. So it would be useful to examine, with the SX-farm data, how the plume inventories differ if these are calculated with and without the lateral data, and then use this as a conversion factor for correcting estimates from areas where only vertical drywell data are available.

The data set spans a very large soil volume. The volume for which predictions would be made is $175(\mathrm{~N}-\mathrm{S}) \times 120(\mathrm{E}-\mathrm{W}) \times 50$ meters (depth), for a total of slightly over one million cubic meters. Data were available for 22,615 distinct locations in this volume. For reasons that will be explained later, the data were aggregated into averages within each cubic meter that contained data, resulting in 3,958 values that could be used for interpolation or prediction. This seems like a lot of data, yet at the resolution of 1 cubic meter, we see that only $0.4 \%$ of the cells in the total volume actually had data in them, and the remaining $99.6 \%$ of the cells would have their value supplied by geostatistical estimation.

Furthermore, the cells that contain data are not randomly or uniformly dispersed through the volume. The data are confined to horizontal or vertical strings of cells, with the consequence that a large fraction of the cells in the total volume can be more than 5 meters from the nearest data location. Figure 2 shows a histogram of numbers of cells a given distance from the nearest data location.

The combination of large prediction volume, large numbers of data locations, and large fraction of the prediction volume being distant from data locations, strains the predictive ability of geostatistical theory, and strains the computational machinery for implementing the geostatistics. Accordingly, there was a premium on using credible geostatistical methods that were, to the extent possible, simple, transparent, and robust, both with respect to the calculation of point estimates and with respect to the quantification of uncertainty. The importance of quantification of uncertainty in this application meant that a more complex and sophisticated methodology with a potential for slightly improving the point estimates would not be desirable if it clouded the calculation of the uncertainty (confidence) bounds.

In this report we document the geostatistical approach used in the SX farm analysis, and explain the rationale for the methodological choices that were made. 


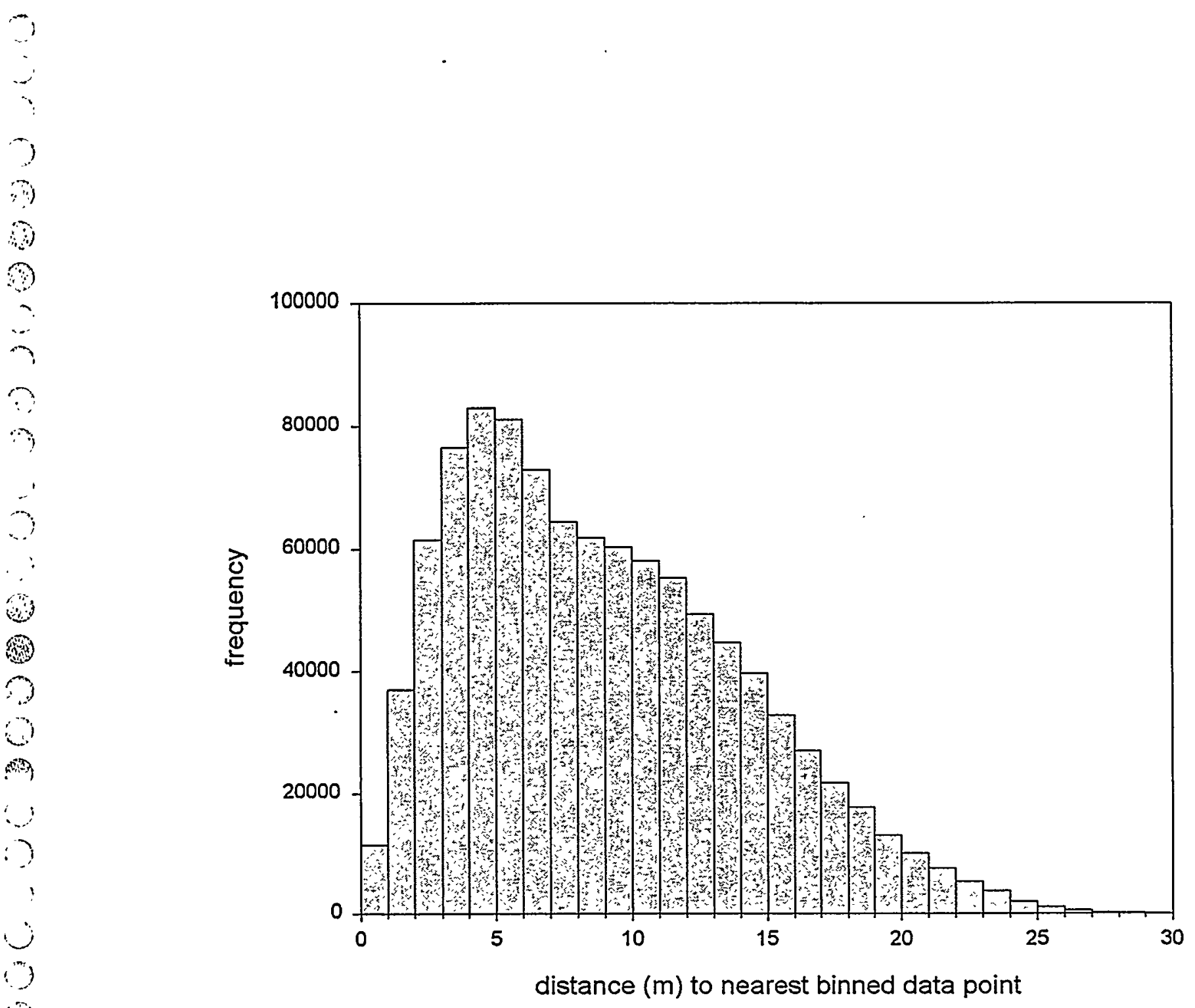

Figure 2:

\section{STANDARDIZATION IN GEOSTATISTICAL PRAC- TICE}

\subsection{Background}

Geostatistics is a relatively new statistical discipline. Mathematically, it is an extension of time series analysis. Parameter estimation for time series analysis suffers a severe degrees-of-freedom problem: the number of time lags at which the process exhibits an influence is generally large relative to the number of data points. This same problem exists in geostatistics where the number of separation distances at which influence can occur is generally large relative to the number of data points. And in geostatistics the problem can be more severe because of 



\subsection{MSU Comparison Study}

In a study to follow through on Englund's question about consistency of results between individual experts, the Environmental Statistics Group at Montana State University, in a cooperative agreement with DOE-Idaho Operations Office, undertook a comparison of popular kriging software in 1998.

The MSU cooperative agreement with DOE-Idaho has spanned several years, and has had geostatistics as one of its consistent themes. This geostatistics project has involved several outside authorities on kriging, including Subhash Lele (then from Johns Hopkins University, now at University of Alberta), John Kern (Western Ecosystems Technology Inc.) and Steve Cherry (then from Montana Technical College, now at Montana State University Math. Dept.), in addition to the Environmental Statistics Group participants, Bert Harting, Chris Wright, and the Principal Investigator. This effort has generated 5 publications related to kriging, in the peer-reviewed literature, and a 6'th submitted (Curriero \& Lele, 1999; Das \& Lele, 1999; Heagerty \& Lele, 1998; Lele, 1995; Lele, 1998; and Lele \& Das, 2000).

In the 1998 software comparison study, the packages examined closely were GEOEAS, GEOPACK, SURFER, S-PLUS and GS+. Our diagnosis revealed some substantial differences among some of the packages (Table 1). Of course, user choices could also cause major difference in results. Three of the programs, SURFER, S-PLUS, and GS+, were sufficiently "automated" that, given a data set, they can produce a kriging prediction using automatically supplied defaults for all the settings. To get a clearer picture of the behavior of these three programs in the hands of a "naive" user, we ran them, in this fully automated way, on a common data set (from a real site at Oak Ridge). The results were remarkably different between the packages. A summary of this study is provided in the Appendix, including example figures of different prediction maps, from the same data, using some of the respective software packages.

An equally disturbing feature revealed in this study was that some aspects of the kriging results generated by the software packages were physically quite unreasonable-most notably the prediction of moderately high contamination at a considerable distance from the known contamination, and this moderately high prediction was located beyond points where data showed low contamination. The mathematical reasons for this un-physical behavior are known: at sufficient distance from data locations, the kriging predictor converges on the data mean when using ordinary kriging (which is the type of kriging the packages carry out). So if the sampling concentrated on the main hot spots, and basically terminated when 
Table 1: Summary of the major features of the geostatistical software packages examined for the kriging comparison project.

\begin{tabular}{|c|c|c|c|c|c|}
\hline & S-PLUS & GEOEAS & GEOPACK & SURFER & GSt \\
\hline \multicolumn{6}{|l|}{ GENERAL } \\
\hline distribution & commercial & public domain & public domain & commercial & commercial \\
\hline OS/interface & Windows (command) & DOS & DOS & Windows & Windows \& DOS \\
\hline data I/O & free-form allowed & Geoeas format & Fortran-style & Excel-style & Excel-style \\
\hline \multicolumn{6}{|l|}{ VARIOGRAM } \\
\hline available models & Sph, gaus, exp, lin & Sph, gaus, exp, lin & Sph, gaus, exp, lin, pow & $\begin{array}{l}\text { Sph, gaus, exp, lin, } \\
\text { hole eff, quad* }\end{array}$ & $\begin{array}{l}\text { Sph, gaus, exp, lin, } \\
\text { linear to sill }\end{array}$ \\
\hline auto model fit? & yes & no & yes & Yes (linear model) ${ }^{*}$ & yes \\
\hline default no lags & 20 & $20(1-24)$ & $\mathrm{NLAG}=12(2-99)$ & ? (no control) & 10 \\
\hline $\begin{array}{l}\text { default max dist for } \\
\text { computation }\end{array}$ & $1 / 2$ maximum range & $\begin{array}{l}\text { entire data range * } \\
\text { (max Euclidean dist) }\end{array}$ & SQRT $(d x * d x+d y * d y) / 2$ & $?$ (no control) & $80 \%$ max distance \\
\hline default lag width & $\max$ dist/20? & max interpair dist $/ 20$ & $\begin{array}{l}\mathrm{BLAG}= \\
\mathrm{SQRT}(\mathrm{dx} * \mathrm{dx}+\mathrm{dy} * \mathrm{dy}) / 24\end{array}$ & ? (no control) & $\left(.80^{*} \max\right.$ dist $) / 10$ \\
\hline \multicolumn{6}{|l|}{ KRIGING } \\
\hline \multicolumn{6}{|l|}{ Krige Types } \\
\hline ordinary/simple & both & both & ordinary only & ordinary only & $?$ \\
\hline point/block & point only & Both (default = point) & both & point only & Both \\
\hline grid vs nongrid est & both & grid only & both & grid or interpolate & Both \\
\hline \multicolumn{6}{|l|}{ Search defaults } \\
\hline search radius & $?$ (no control) & $1 / 4 \max$ distance & RMAX = range & default=all pts & Max interpoint dist \\
\hline \# sectors & ? (no control) & 1 & NA & 1 & 1 \\
\hline max no neighbors & $?$ (no control) & $16(1-24)$ & 10 & All pts (if $<200)$ & 16 \\
\hline min no neighbors & $?$ (no control) & $8(1-16)$ & 1 (can't reset) & 1 & ? (can't set) \\
\hline \multicolumn{6}{|l|}{ Grid Parameters } \\
\hline default grid size & $30 \times 30$ & $10 \times 10(\max =100 \times 100)$ & $10 \times 10(\max =?)$ & $50 \times 49$ & Variable \\
\hline default grid width & NA & entire data range & entire data range & Longest axis & Entire data range \\
\hline
\end{tabular}

* Surfer provides no variogram modeling facility. It provides default parameters for a linear variogram that are used for kriging or the user can specify other models \& parameters 
the boundary of the hot spot was identified with a zone of samples showing low concentration, the prediction field can show a strange-looking bias: where the prediction field extends too far beyond the locations sampled, it will have the appearance of well-defined hotspots, bounded by zones of low contamination, but located on a background of medium contamination.

Experts have several ways to suppress this unreasonable behavior: detrending is the most common. But detrending opens up a whole new set of choices to the user, potentially even more complex than the choices in the kriging operation itself. The premise of detrending is that some mechanism other than the kriging model is responsible for the large scale pattern. The approach is to model that mechanism and large scale pattern by virtually any method, and then apply kriging to the residual. So the option of detrending does not solve the problem of satisfactory standardization, unless there were consensus on what non-kriging model should be used for the large scale pattern. Of course, the absence of consensus about the mechanistic model in most applications is what made kriging seem so attractive in the first place.

\subsection{Kriging Choices and Prediction Uncertainty}

Within the kriging paradigm, even for "entry level" kriging, choices need to be made about the variogram model, and more choices need to be made about how the variogram parameters will be estimated from data. The popular kriging software offers choices among linear, spherical, exponential, and Gaussian variogram models. These software packages estimate the parameter values for the chosen variogram model by "fitting" the variogram to the pattern of covariance decay with distance in the data. Generally there are too few pairs of data points for any particular separation distance, so in order to stabilize the observed scatter plot of covariance against distance, the covariances for pairs of points are assigned to separation distance intervals (called "bins"), the values within each bin are averaged, and then the variogram is "fit" to the bins. This is an ad hoc procedure, the number of bins, the bin intervals, restrictions on the minimum number of pairs per bin, etc. are all choices, but these do affect the result.

The central kriging model is well developed as a methodology for making regression-like predictions of values at unobserved locations from data at observed locations assuming, the variogram is known. The standard "kriging prediction error" calculations are based on the same assumption-that the true variogram is known. But of course, the true variogram is not known: it has to be esti- 
mated somehow, and uncertainties associated with that estimation add to the actual uncertainty about the kriging predictions. This fact is known among the mathematical experts in the field. The comprehensive standard text of geostatitical computation, by Deutsch and Journel (1998) frankly states (p 15): "Kriging variances are usually not measures of local estimation accuracy." But standard methodology and standard software does not account for this. The problem has motivated some mathematical research into Bayesian methods to fold the error resulting from variogram estimation into the reported prediction error (Handcock and Stein, 1993; Cui, et al. 1995), but this work is still in the stage of theoretical methods development, and it is computationally very costly for large data sets.

A further limitation of the standard "prediction error" calculated by the popular software packages is that it is an error that applies to a point in space. One can draw a map (e.g., contouring) of the magnitude of the "point-prediction-error" but this is insufficient for understanding errors in quantities, such as block averages, gradients, or boundary locations, that may be important for decision-making or regulatory purposes. The reason that the map of point-prediction-error cannot serve adequately in this role, is that the errors at different locations are not independent of one another under the kriging model. The same spatial pattern of correlation that is the basis for the kriging prediction also applies to the kriging prediction error. For example, an overestimate at a particular location is very likely to be associated with overestimation in the entire neighborhood of that location. For this reason, the local point-prediction-errors within a block are not likely to be correctly represented by simply summing over a block in an attempt to estimate the error bounds on the inventory for the block. The pattern of spatial correlation in the error needs to be taken into account for calculating the error in any quantity that involves more than one "point" in space.

Generally, the only family of techniques adequate to deal with error estimation for quantities of actual interest is "conditional simulation" (Journel, 1974). Such methods have gained acceptance as the leading edge approach for dealing with prediction error in groundwater and soil contamination geostatistical estimates (Gotway, 1994). These methods are also the natural framework for using decision theory in a geostatistical analysis of a contaminated site (Freeze, et al., 1990; Freeze et al., 1992).

Unfortunately, conditional simulations are computationally extremely expensive, and they require custom programming to represent the details of the decision quantity for which the error is to be calculated. A further awkwardness is that the underlying model that drives the simulation is different from the most popular 


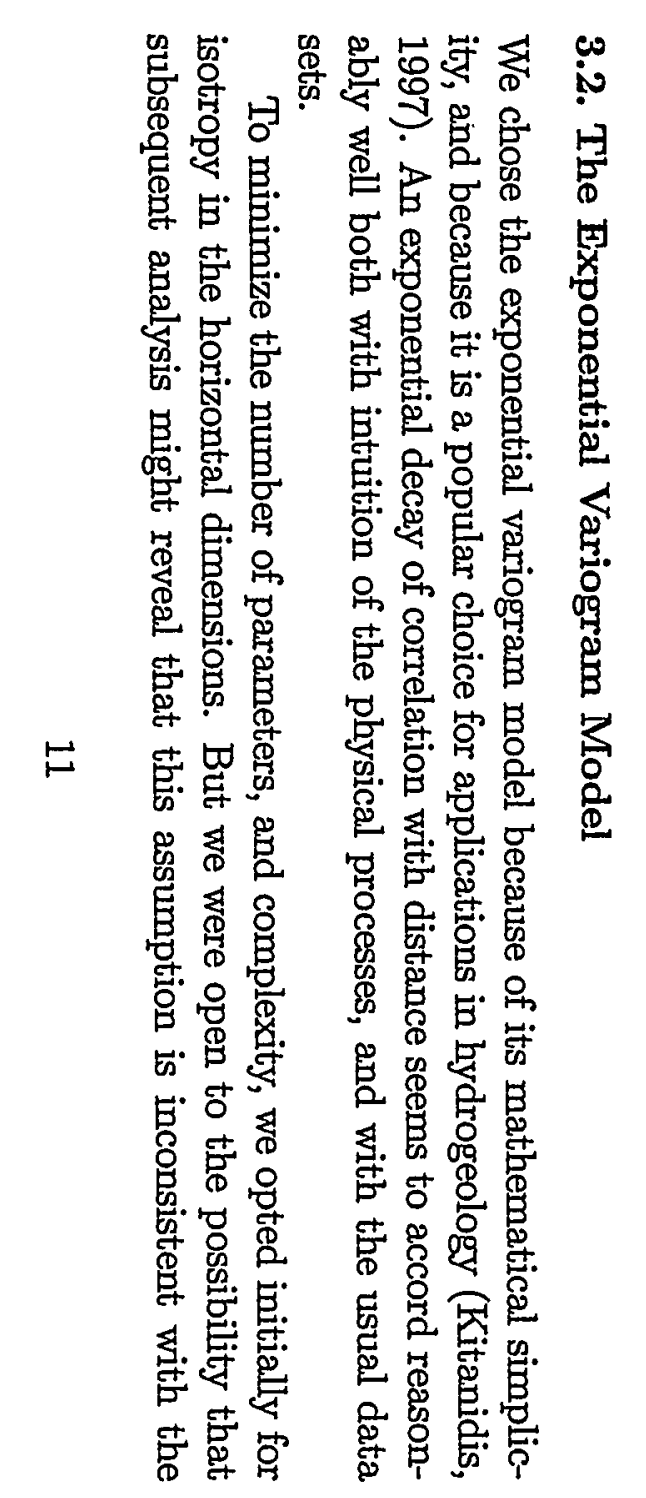

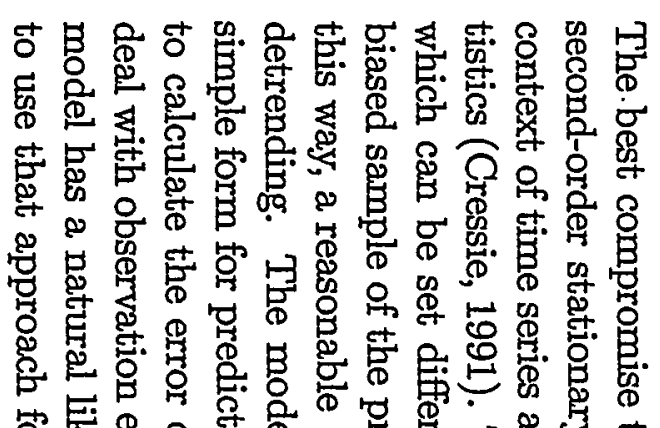

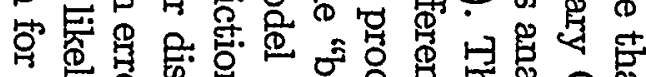

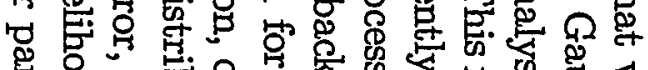
恶

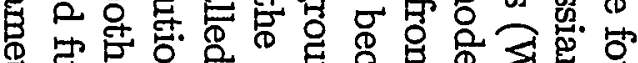
品

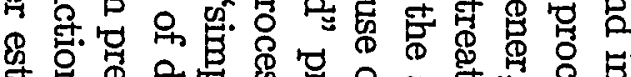

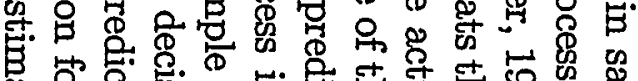

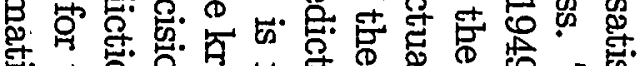

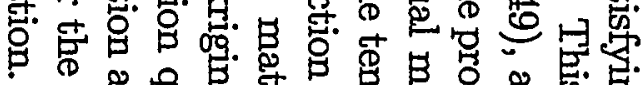

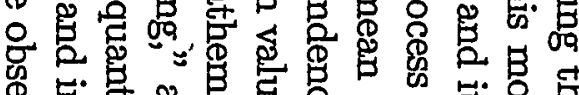

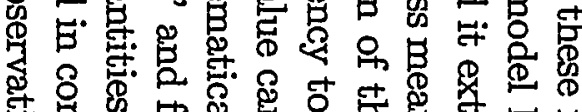

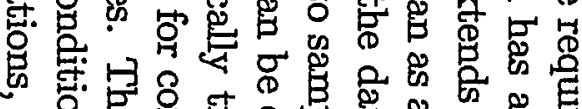

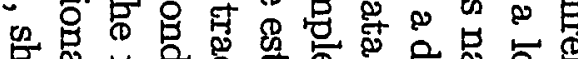

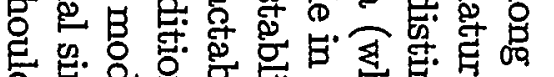

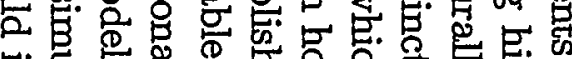
푸의





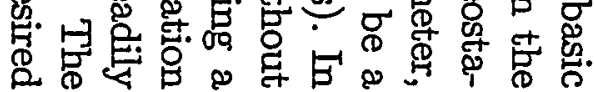

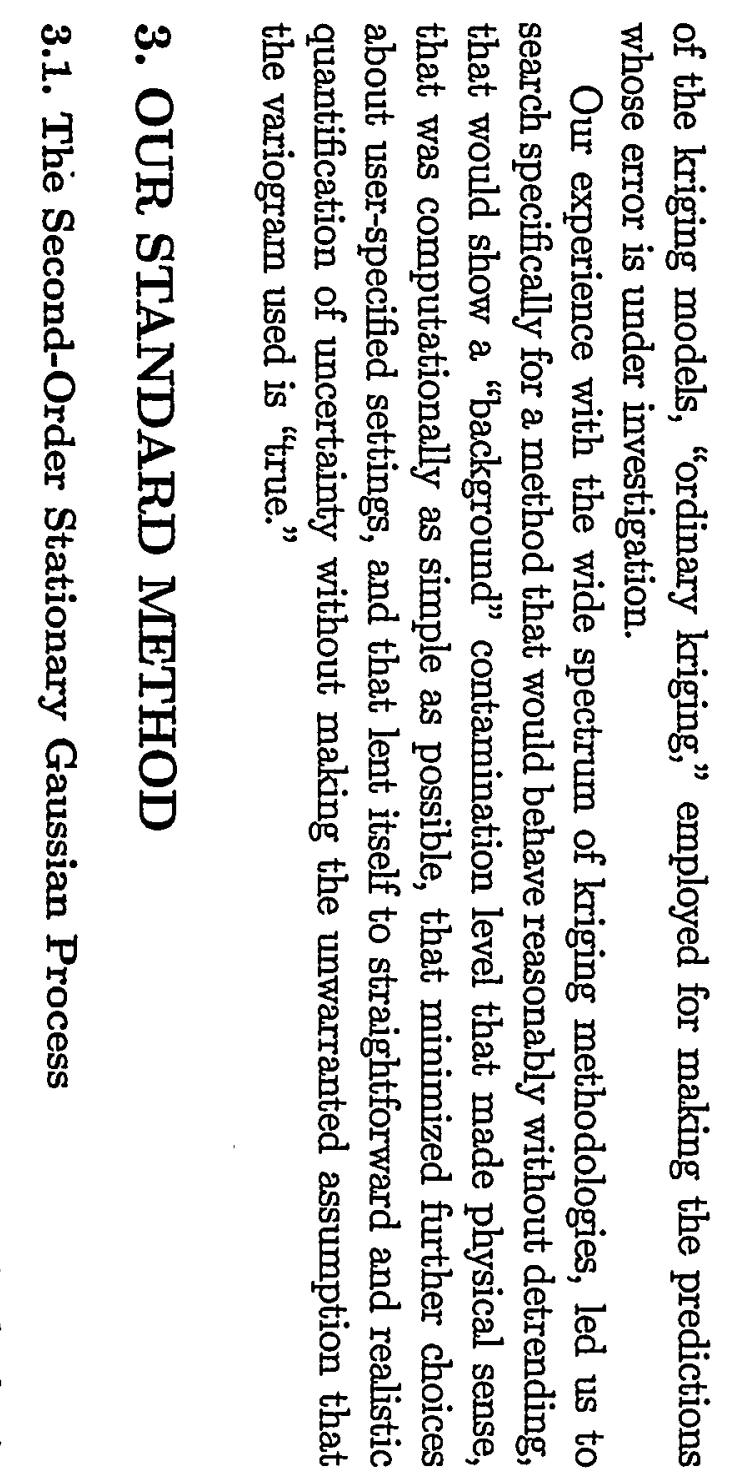


data. (In the actual event, it did not, and we stayed with horizontal isotropy.) Because the movement of contamination in the vertical dimension in the vadose zone clearly is governed by physical mechanisms that are quite different from those controlling horizontal dispersion, we set a separate parameter for correlation with distance in the vertical dimension.

\subsection{Parameter Estimation}

This model structure left us with only 5 parameters to determine:

1. the process mean,

2. the process variance,

3. the rate of correlation decay with distance in the horizontal,

4. the rate of correlation decay with distance in the vertical,

5. the measurement error variance.

The process mean was set to duplicate the observed "background" in the tank farm. The gamma logging data showed a distinctly bi-modal distribution, with a normal mode at $25 \mathrm{cps}$, and a broad, definitely non-normal, mode centered between $e^{14}$ and $e^{15}$. Because of the scale, the two modes can not be shown clearly in the same histogram: Figure 3 shows the lower mode, and Figure 4 shows the upper mode. We took the distinct mode at $25 \mathrm{cps}$ to be the local background, and used this value for the process mean. The shape of the larger mode indicated that some transformation of the data was required, and we chose log transformation, which resulted in a much more normal-looking distribution than the original histogram.

The variance of the log-transformed means of the averaged counts in each 1-meter-cubed cell with data was taken as the process variance. This split the difference between two extreme assumptions about the process: one extreme would have doubled the range because the large mode described a distribution that was almost entirely above the nominal process mean value, the other would have shrunk the process variance to be consistent with a large sea of background.

In the exploratory analyses which have been done so far, we tentatively set the measurement error variance to zero. When we have the opportunity to study the data more closely, we can estimate measurement error variance from the replication variance of multiple re-measurements. This analysis of replication variance 


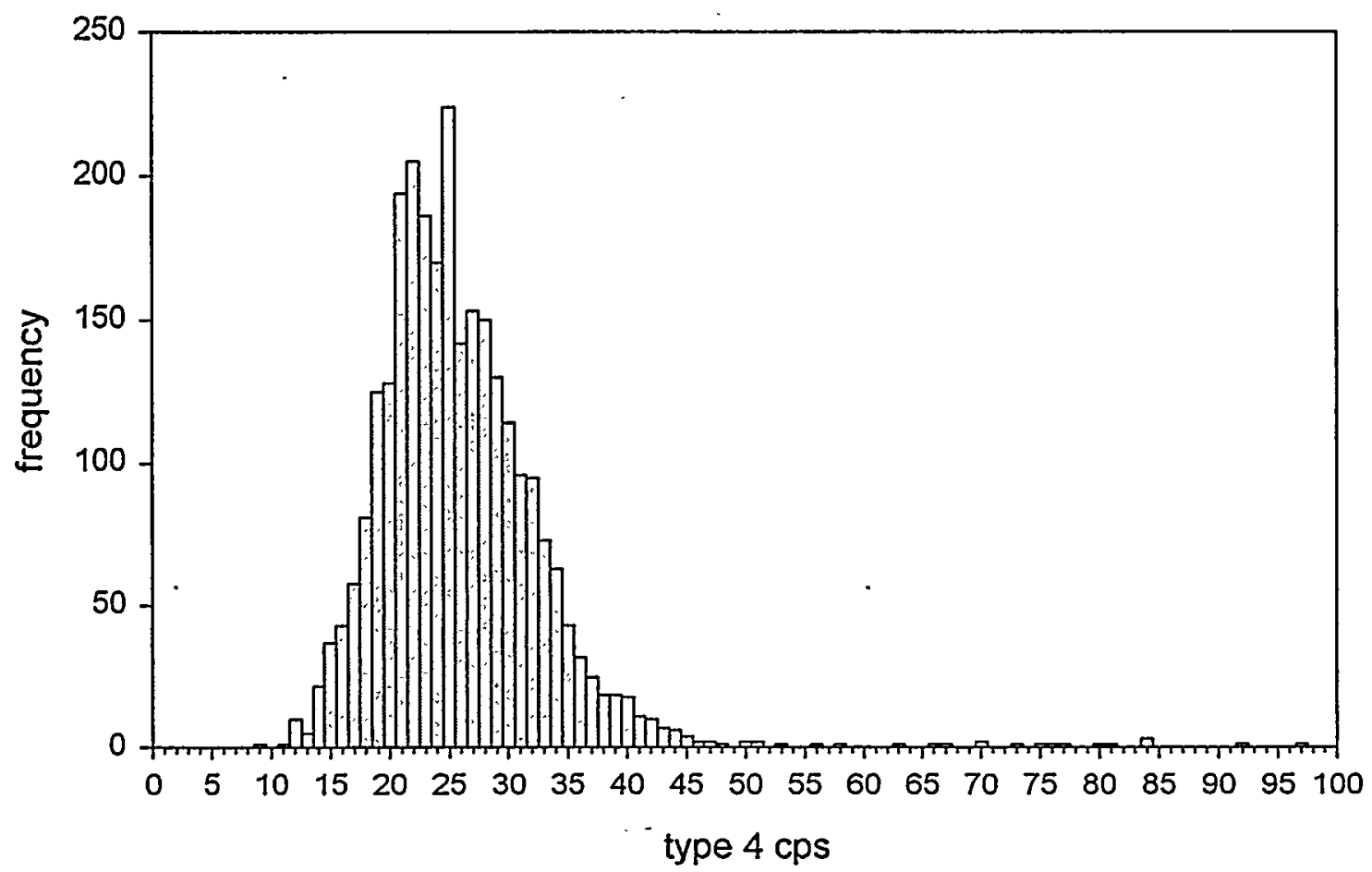

Figure 3:

is too complicated to have been done during our initial exploratory investigation, because the measurement error must be estimated separately for each of 6 or 7 different instruments that have been used in the SX-farm monitoring.

This left only the range of influence parameters (horizontal and vertical) remaining to be estimated. These describe the rate at which correlation decays with increasing distance (in each direction). One conventional way (familiar to physicists) to report this parameter is as the $e$-folding distance: the separation distance at which the correlation between local contamination levels (true values, without measurement error) is $1 / e$ (roughly $37 \%$ ).

Because our interest is in predictions and accurate estimation of prediction error, we chose to bypass all the usual variogram estimation procedures of "binning" and "fitting" which focus on fitting the variogram rather than fitting the prediction surface. Instead we employed jack-knife cross-validation, in the kriging model itself, adjusting the two free range-of-influence parameters to minimize the jack-knife residual. Because this operation was carried out within a model that 




Figure 4:

already embodied all the other assumptions we had made, and parameters we had set, the jack-knife parameter estimation had the further advantage of optimizing the two free parameters in context, so that any mis-specifications could be compensated to the extent possible. Similarly, the jack-knife prediction error would be representative of the actual prediction errors in this application.

\section{APPLICATION TO THE SX-FARM DATA SET}

Over the period of time since October 1998, when this project began (initially as a methods development case study, under the DOE-Idaho cooperative agreement), the data set gradually "grew" as more kinds of data (such as the laterals, and the 
soil core samples) were retrieved from archives, and as we iterated with experts at Hanford to obtain consensus on the intricate issues of interconversion between measurements with several different instruments, and as the focus of interest expanded from the main plume under tanks 108 and 109, to include other plumes, such as those under 107 and 115 . The latter two plumes were poorly characterized with the data that were initially available, but became more accessible to analysis with the inclusion of data from the laterals.

The data set that we consider complete and consistently cross-calibrated was finalized only this past July. That data set has not yet been documented, and has not yet been analyzed according to the parameter estimation scheme described here.

The parameter estimates that we do have were obtained earlier, using the methodology described above, but using only about half the data that are now in hand. The parameter estimates from that effort gave an $e$-folding distance of about 5 meters. The residual response surface from the jack-knife cross validation was steep in the direction of smaller values of these parameters, but had a shallow slope in the direction of larger parameter values. This indicates that, at least for this subset of the data, the lower bound of the $e$-folding distance was securely established, but there was uncertainty about the upper bound that was reasonably consistent with the data. The $e$-folding distance basically defines the distance over which contamination values exhibit strong influence (strong correlation), and similarly this is the distance over which data values are combined with high weights (more or less like averaging or smoothing) in the kriging prediction.

An example surface of jack-knife residual sum of squares cross-validation error, from data on the six-tank area around 108 at a time when we had data from only one set of laterals, is given in Figure 5.

A later analysis of data just from the laterals is shown in Figure 6. An analysis to test for influence of kriging search radius on the jack-knife cross-validation parameter estimation with the lateral data is shown in Figure 7. This last analysis showed that search distances greater than $1.5 \mathrm{x}$ the $e$-folding distance were unnecessary. The search radius is the distance within which data values are used to make a prediction (interpolation), and the smaller the distance the quicker the calculation.

One more feature of the jack-knife cross-validation methodology is worth noting. The procedure can jack-knife out data blocks of any defined size, creating the option for fine tuning the parameter estimation to optimize the predictive power over a selected range of distance, and to fine tune the estimate of prediction error 


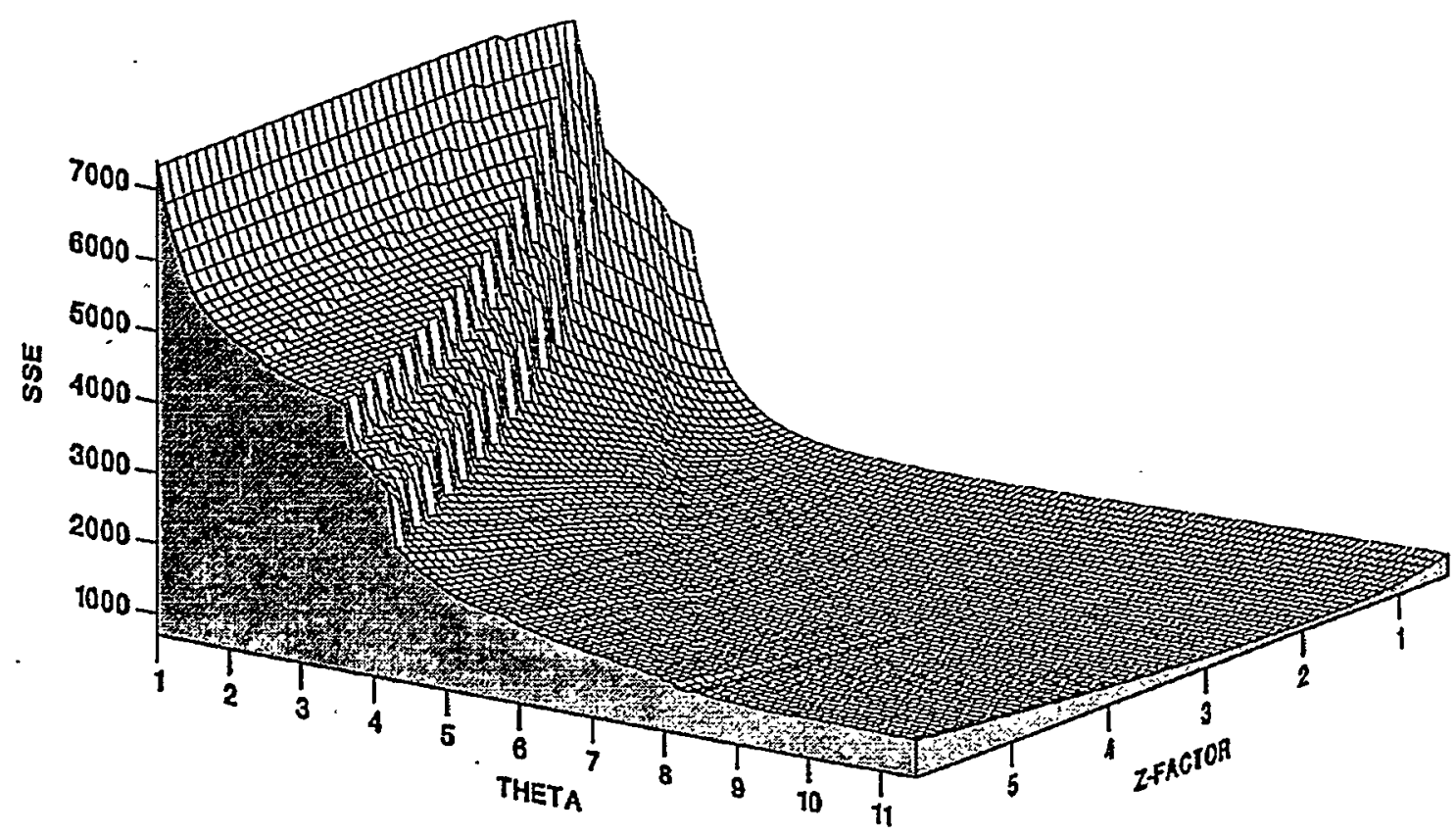

Figure 5: Jack-knife cross-validation residual sum of squares plotted against the horizontal e-folding distance(theta) and the multiplier that rescales the vertical distance (z-factor) for the same e-folding distance.

in that range. This is very useful in the present application, since from the known geometry of the data locations, we know what are the most important distances over which we will need to interpolate. 




Figure 6: Jack-knife cross-validation residual sum of squares plotted against the horizontal e-folding distance(theta). 


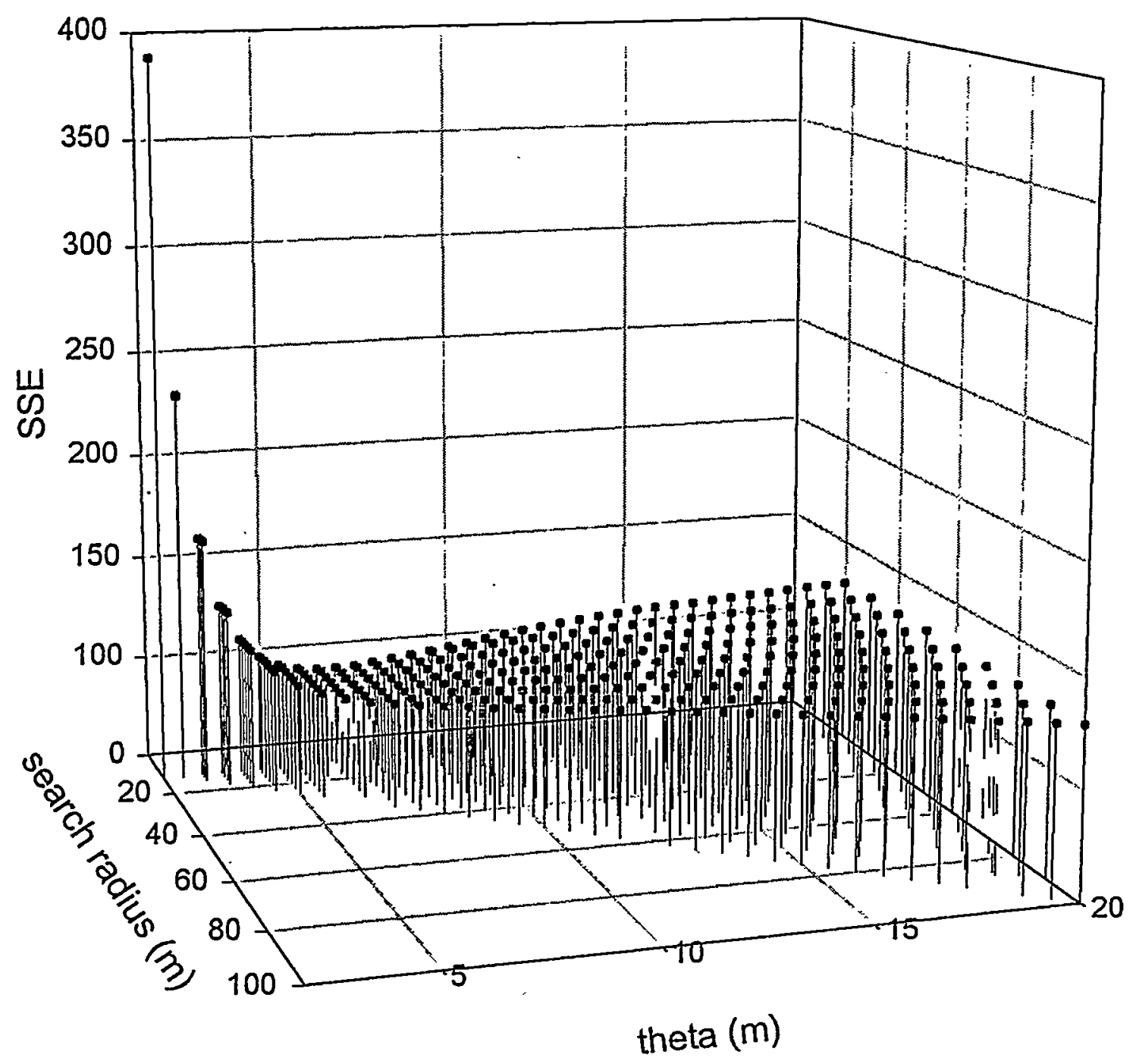

Figure 7. Jack-knife cross-validation residual sum of squares plotted against the horizontal e-folding distance(theta) and the search radius. 


\section{MATHEMATICAL REPRESENTATION}

\subsection{Notation}

$k \quad$ number of dimensions

$n \quad$ number of locations with observations

$m \quad$ number of locations where value of process is to be predicted

$\underline{y} \quad \mathrm{n}$-element vector of observed values, indexed by location, where $y_{i}$ is observed value at location $i$, and where $i$ is a $k$-element vector of coordinates in a cartesian space

$\underline{x}$ n-element vector of unobserved values, of the underlying process, indexed by location, where $x_{i}$ is value of the process at location $i$, and where $i$ is a $k$-element vector of coordinates in a cartesian space

$\sigma_{x}^{2} \quad$ variance of underlying process generating values of $x$ $\sigma_{\epsilon}^{2} \quad$ variance of observation error

$\mu_{x} \quad$ mean of underlying process generating values of $x$

$C_{w z} \quad$ covariance matrix of random vectors $w$ and $z$, where $c_{w z}(i, j)$, element $(i, j)$ of this matrix, is the covariance between element $i$ of vector $w$ and element $j$ of vector $z$

$\underline{\alpha} \quad k$-element vector of scale factors to rescale distance from origin along each dimension for purposes of computing a distance measure which weights each dimension separately

$d_{\underline{\alpha}}(i, j) \quad \alpha$-scaled distance between locations $i$ and $j$ computed as a euclidean distance after rescaling the distance from the origin along coordinate $h$ by $\alpha_{h}$

\subsection{Model of the Underlying Process}

$$
\begin{aligned}
& \underline{x} \sim N\left(\underline{\mu_{x}}, C_{x x}\right) \\
& x_{i} \sim N\left(\mu_{x}, \sigma_{x}^{2}\right)
\end{aligned}
$$

5.3. Spatial Structure of the Underlying Process

$$
\begin{gathered}
c_{x x}(i, j)=f\left(d_{\underline{\alpha}}(i, j)\right) \\
f\left(d_{\underline{\alpha}}(i, j)\right)=\sigma_{x}^{2} e^{-d_{\underline{\alpha}}(i, j)}
\end{gathered}
$$




$$
\begin{gathered}
c_{x x}(i, i)=\sigma_{x}^{2} \\
d_{\underline{\alpha}}(i, j)=\left(\sum_{h=1}^{h}\left(\frac{i_{h}}{\alpha_{h}}\right)^{2}\right)^{\frac{1}{2}}
\end{gathered}
$$

5.4. Relating Observations to the Underlying Process

$$
\begin{gathered}
y_{i}=x_{i}+\epsilon_{i} \\
\epsilon_{i} \sim N\left(0, \sigma_{\epsilon}^{2}\right)
\end{gathered}
$$

5.5. Spatial Structure of the Observations

$$
\begin{aligned}
& c_{y y}(i, j)=c_{x x}(i, j) \\
& c_{y y}(i, i)=\sigma_{x}^{2}+\sigma_{\epsilon}^{2}
\end{aligned}
$$

5.6. Joint Spatial Structure of Observations and the Underlying Process

$$
\begin{gathered}
c_{x y}(i, j)=c_{x x}(i, j) \\
c_{x y}(i, i)=\sigma_{x}^{2}
\end{gathered}
$$

5.7. Joint Distribution of Observations and the Underlying Process

$$
\begin{gathered}
\underline{v}=\left(\frac{\underline{y}}{\underline{x}}\right) \\
C_{v v}=\left(\begin{array}{ll}
C_{y y} & C_{y x} \\
C_{x y} & C_{x x}
\end{array}\right)
\end{gathered}
$$


5.8. Conditional Distribution of the Process, Given the Observations

$$
\begin{gathered}
(\underline{x} \mid \underline{y}) \sim N(\underbrace{}_{(\underline{x} \mid \underline{y})}, C_{(\underline{x} \mid \underline{y})(\underline{x} \mid \underline{y})}) \\
\underline{\mu_{(\underline{x} \mid \underline{y})}}=\underline{\mu_{x}}+C_{x y} C_{y y}^{-1}\left(\underline{y}-\underline{\mu_{x}}\right) \\
\left.C_{(\underline{x} \mid \underline{y})(\underline{x} \mid \underline{y})}\right)=C_{x x}-C_{x y} C_{y y}^{-1} C_{y x}
\end{gathered}
$$

\subsection{Likelihood Function}

$$
\ln \left(L\left(\mu_{x}, \sigma_{x}, \underline{\alpha}\right)\right)=-\frac{n}{2} \ln (2 \pi)-\frac{1}{2} \ln \left(\left|C_{x x}\right|\right)-\frac{1}{2}\left(\underline{x}-\underline{\mu_{x}}\right)^{T} C_{x x}^{-1}\left(\underline{x}-\underline{\mu_{x}}\right)
$$

\subsection{Computational Demands and Computational Strategy}

For a data set with observations at $n$ locations, the kriging equation requires inversion of an $n \times n$ matrix. A point kriging prediction requires, at each new location to be interpolated, two applications of an $n \times n$ matrix in multiplication to an $n$-element vectors. So, overall, a simple point kriging prediction, without error analysis, for a field of $m$ points to be predicted, exacts a computational cost proportional to $m \times n^{2}$.

Embedding the point kriging in a.jack-knife cross-validation for parameter estimation, further multiplies the computational cost by the number of combinations of parameter values explored.

Conditional simulation of a field of $m$ points; conditioned on a data set of $n$ points, requires inversion of an $(n+m) \times(n+m)$ matrix. Each realization of the field requires generation of $(n+m)$ normal variates and application of an $(n+m) \times(n+m)$ matrix in multiplication to an $(n+m)$-element vector. Generally, several thousand realizations will be required for satisfactory characterization of the distribution of a decision quantity. If $\ell$ is the number of realizations, conditional simulation exacts a computational cost proportional to $\ell \times(m+n)^{2}$.

Two bottlenecks, therefore, can limit the feasibility of the application. One is the size of the matrices that must be inverted; the second is the brute number of multiplication operations. The Cholesky decomposition offers efficiency and stability for the inversion of these positive-definite real symmetric matrices (Ripley, 1981; Ng \& Peyton, 1993). The brute number of multiplications can be controlled by a strategy of limiting the number of prediction points $m$ to the minimum needed to really answer the question (rather than just densely filling the field to 
get an attractive "picture"), and limiting the number of predictor points used for each prediction to a neighborhood of a small multiple of the $e$-folding distance around the location to be predicted.

\section{RESULTS AND FUTURE DIRECTIONS}

The gamma monitoring data set for SX farm is unusually rich. For the 15 tanks in this farm, we have data from 99 vertical far-perimeter drywells positioned the way most monitoring drywells are in the 200 area, plus 24 lateral drywells, and 16 soil cores from the tank close-perimeter (closer to the tank than the vertical drywells). For most of the vertical far-perimeter drywells, we have recent spectral gamma data. For a sampling of the spectral gamma readings, we have counts attributed specifically to the Cs-137 peak.

We have gone to some effort to cross calibrate among readings from different gross gamma instruments (that differ in their sensitivity and shielding), and spectral gamma, spectral gamma Cs-137, and Cs-137 measurements from soil core samples. With this cross calibration, and decay correction for the different dates of the measurements, we can merge gross gamma logging data, and soil core sample Cs-137 measurements from earlier periods, to complete the data for locations that do not have recent spectral gamma measurements (or where the spectral gamma saturated).

Most of the other tank farms do not have similar data richness. The gamma monitoring data for most farms is confined to vertical drywells. Our preliminary results have already shown that the lateral data in SX farm make a considerable difference to the reconstructed plume and the estimate of plume inventory. Figure 8 shows a 6-tank subset of the farm, viewed from the East, with tank 108 in the center front. The lateral wells show as dark green horizontal lines under some of the tanks. The $e^{8} \mathrm{cps}$ (in probe type-4 units) iso-surface of the reconstruction of the plume from tanks 108 and 109 shows as the irregular shape. The reconstruction based just on the vertical dry-well data appears as several disconnected opaque gray blobs; the transparent purple extension, that connects all the blobs into a single larger plume is the reconstruction that includes the lateral data with the vertical dry-well data. Figure 9 shows the same area from above, with the tanks transparent. We see the importance of the lateral data to the reconstruction of a coherent plume geometry. 


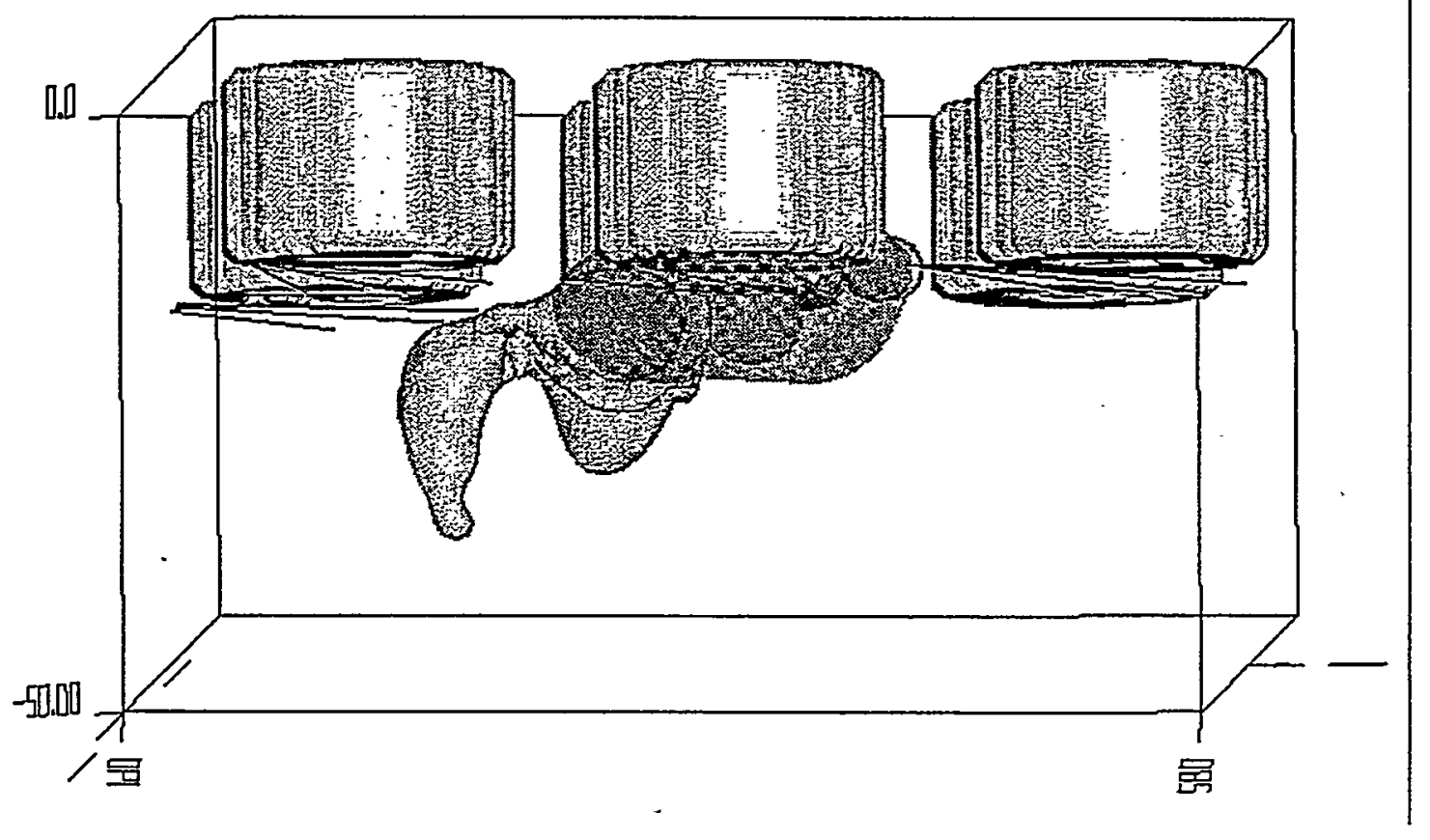

Figure 8.

\subsection{Visual Inspection and Interpretation of Results}

Geostatistical analysis, such as 3-D kriging, can be applied to the merged data set, to obtain interpolation estimates of the geometry, and concentration contours, of the gamma contamination plume under the SX farm tanks. Because the features of interest are 3-dimensional, and the shapes are complicated, it can be difficult to obtain an adequate understanding from static 2-dimensional pictures. The 3 -d visualization software, VIS5D, developed for NASA, provides a convenient means for visual inspection and interpretation. This software allows rotation of the image, under joystick-like mouse control, which gives the user an excellent 3-D sense of the image. Combining the rotation with slice, zoom, and multiple layer representation allows us to show the estimated plume configuration in relation to the tanks, geographic strata, and other structure, and to show the magnitude of the uncertainty in this interpolation at each location. 


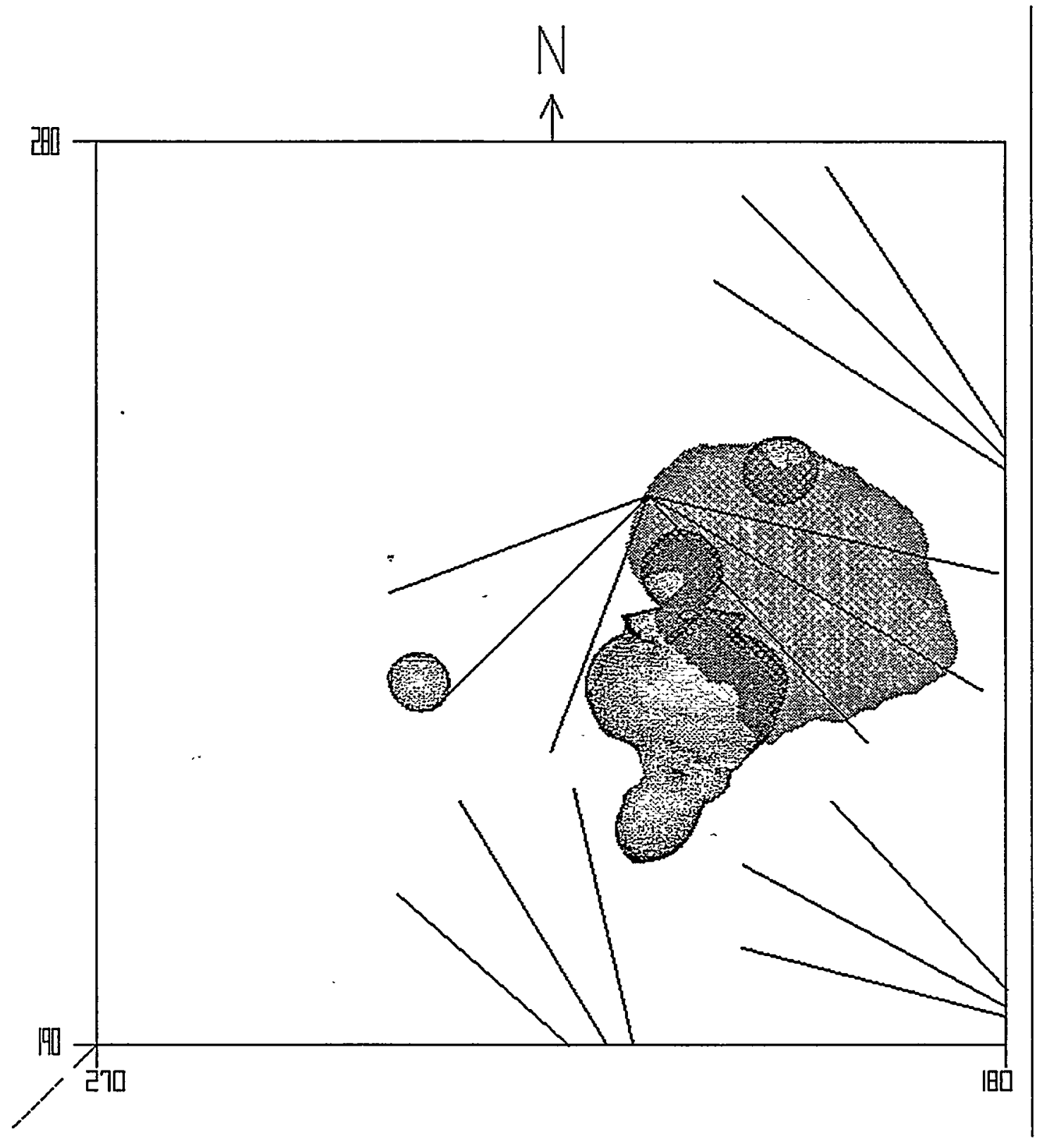

Figure 9. 


\subsection{Calibrating Plume Estimates for Data Sets Without Laterals}

The composite Cs-137/gamma data from SX farm show definite contamination plumes under 4 of the tanks: 107, 108, 109, and 115. For all 4 of these tanks, we have vertical drywell and lateral drywell data. For two of these tanks (108 and 115) we also have tank close-perimeter soil core sample data. This presents an opportunity to test the power and sensitivity of vertical drywell logging alone as a means to detect and quantify contamination plumes. In such an exercise, the geostatistcal plume reconstruction from the merged data set, including laterals and close-perimeter soil cores, would be used as "ground truth," to investigate how accurately the plume can be reconstructed from vertical drywell data alone.

We have already noted, qualitatively, that the large plume under tanks 108 and 109 shows up clearly in the vertical drywell data (though it looks different when the lateral data are added). The smaller plumes under tanks 107 and 115 are not clearly delineated by the vertical drywell data, and need lateral and/or close-perimeter soil core data, closer to the actual plume, in order for the plume to be obvious.

The point of a plume reconstruction calibration analysis would be to refine and quantify these observations, (1) to determine how large a plume inventory has to be in order for it to be detected with confidence using vertical drywell data alone, and (2) to calibrate the difference in plume inventory estimates using all the data versus vertical drywell data alone, to establish the reliability of a corrected estimate based on vertical drywell data alone.

These calibrations would be very important for interpreting the geostatistics from other tank farms which have only vertical drywell data.

\subsection{Correlating Plume Inventory Estimates and Leak Inventory}

The ability to reconstruct plume Cs- 137 inventory by geostatistical analysis of gamma logging data presents an opportunity to compare that inventory estimate with an independent estimate based on leak volume and tank contents composition at the time of the leak. There are several sources of such leak inventory estimates.

This comparison should set some bounds on the reliability of the plume inventory estimates, particularly as a function of data density under the tank in question. This analysis would prove valuable for making decisions about additional future sampling for purposes of improving the confidence in estimates of plume inventory under specific tanks where the present uncertainty poses a problem. 


\section{REFERENCES}

Christakos, G. 1992. Random Field Models in Earth Sciences. Academic Press, San Diego.

Cressie, N. 1991. Statistics for Spatial Data. John Wiley \& Sons, N.Y.

Cui, H., A. Stein, and D.E. Myers. 1995. Extension of spatial information, Bayesian kriging, and updating of prior variogram parameters. Envirometrics 6:373-384.

Curriero, F. and Lele, S. 1999. A composite likelihood approach to semivariogram estimation. J. Agr. Biol. Env. Statist. 4: 9-28.

Das, A., and S.R. Lele. 1999. Robust inference for spatial data under model misspecification. (Submitted to Math. Geol.)

Deutsch, C.V., and A.G. Journel. 1998. GSLIB, Geostatistical Software Library and User's Guide. 2nd ed. Oxford Univ. Press. New York.

Englund, E.J. 1990. A variance of geostatisticians. Math. Geol. 22:417-455.

Freeze, R.A., J. Massmann, L. Smith, T. Sperling, and B. James. 1990. Hydrogeologic decision analysis: 1. A framework. Ground Water 28:738-766.

Freeze, R.A., J. Massmann, T. Sperling, and L. Smith. 1992. Hydrogeologic decision analysis: 4 . The concept of data worth and its use in the development of site investigation strategies. Ground Water 30:574-588.

Gotway, C.A. 1994. The use of conditional simulation in nuclear-waste-site performance assessment. Technometrics 36:129-141 (with discussion 141-161.)

Handcock, M.S., and M.L. Stein. 1993. A Bayesian analysis of kriging. Technometrics 35:403-410.

Heagerty, P.J., and S.R. Lele. 1998. A composite likelihood approach to binary data in space. JASA 93:1099-1111.

Journel, A.G. 1974. Geostatistics for conditional simulation of ore bodies. Economic Geology 69:673-687.

Journel, A. 1986. Models and tools for the earth sciences. Math. Geol. 18:119-140.

Kitanidis, P.K. 1997. Introduction to Geostatistics. Cambridge University Press. Cambridge.

Lele, S. 1995. Inner product matrices, kriging, and non-parametric estimation of variogram. Math. Geol 27:673-692.

Lele, S. 1998. Estimating functions for semivariogram estimation. In, I.V. Basawa, V.P. Godambe, and R.L. Taylor (eds.) Selected Proceedings of the Symposium on Estimating Functions. IMS Lecture Series Monographs, 381396. 
Lele, S.R., and A. Das. 2000. Elicited data and incorporation of expert opinion for statistical inference in spatial studies. Math. Geol. (In press).

Ng, E.G., and B.W. Peyton. 1993. Block sparse Cholesky algorithms on advanced uniprocessor computers. SIAM-Journal of Scientific Computing 1034-1056. Ripley, B.D. 1981. Spatial Statistics. John Wiley \& Sons, N.Y.

Wiener, N. 1949. Extrapolation, Interpolation, and Smoothing of Stationary Time Series. Technology Press, MIT, and John Wiley \& Sons, N.Y. 


\title{
APPENDIX \\ THE 1998 KRIGING SOFTWARE \\ COMPARISON STUDY*
}

\author{
I. Evaluation of Geostatistical Software Packages \\ II. Kriging Software Packages
}
A. GEOEAS
B. GEOPACK
C.SURFER
D.S-PLUS

III. Comparing Kriging Results Using S-Plus and Fortran Simulations

IV. How the Software Comparison Maps were Created

V. Summary of Software Evaluation Results

* New releases of some of the software have appeared since that time. 


\section{EVALUATION OF GEOSTATISTICAL SOFTWARE PACKAGES}

This report provides a preliminary overview of the software packages reviewed for the kriging comparison project. Four packages have been reviewed to date. These include two public domain packages written for DOS (GEOEAS and GEOPACK) and three commercial packages for Windows (S-PLUS, SURFER and GS+). Other packages that will be reviewed for this project include UNCERT (written for the UNIX platform) and a collection of MATLAB kriging routines developed by a collaborator of this project.

Another set of software components in wide use by geostatisticians is GSLIB. This is not a software package per se, but rather a library of Fortran routines which perform a broad assortment of geostatistical operations, including kriging. The algorithms developed for GSLIB routines have been incorporated into a number of other applications, including the S-Plus spatial statistics module.

Section I of this report provides an overview of the five packages examined thus far. Fundamental features of the software are discussed, with particular attention to the variogram estimation and kriging modules. The protocol developed for the software evaluation project requires that the procedures built into the packages be emulated in external simulation routines. In general, we attempted to duplicate the default behaviors of the respective packages as closely as possible. However, in some cases (for example, if the program defaults were impossible or totally arbitrary values), it was necessary to modify the default behaviors of the respective packages. Table 1 at the end of this report provides a summary of the primary features of each package.

Section II and III of the report discusses the methodology employed for comparing the kriging packages. This presentation is necessarily preliminary as the evaluation protocols are still under revision. 


\title{
Kriging Software Packages
}

\section{A. GEOEAS:}

Developer:

Evan Englund

U.S. Environmental Protection Agency

Environmental Monitoring Systems Laboratory

Las Vegas, Nevada 89193-3478

\author{
Allen Sparks \\ Computer Sciences Corporation \\ 4220 S. Maryland Parkway \\ La Plaza B, Suite 408 \\ Las Vegas, Nevada 89119
}

Distributed by:

Center for Subsurface Modeling Support (CsMoS)

http://www.epa.gov/ada/models.html

(also available elsewhere on the web)

Distribution terms: public domain freeware

General Notes:

GEOEAS is perhaps the most widely distributed geostatistical package and is a standard tool for geostatisticians. The main tools are the following: basic univariate statistics (scatter plots/linear regression), spatial correlation analysis of the data with the variogram, model specification of the variogram, cross validation of kriging parameters, 2D estimation using kriging, 2D graphic and contouring tools. A user's manual can be downloaded in postscript and Adobe Acrobat format. Variogram computation may be limited to a subset of the input data by specifying a polygon outside of which no kriging is performed. This software is in the public domain and is available for free download from a number of websites.

Limitations of the Program (partially extracted from AI Geostats homepage):

- no real GIS features (no spatial queries, no GIS functions like buffering, distance analysis)

- graphical outputs not presentation quality

- tools are only dedicated to basic geostatistics

- no automatic variogram modeling 
- kriging predictions at gridded locations only

\section{User Interface}

GEOEAS is a DOS-based program, which is partitioned into a number of separate interactive software tools for performing different functions. The basic tools (or modules) necessary for kriging are the PREVAR, VARIO, and KRIGE tools. The data entry menus are typical DOS style in which the user navigates using enter and/or arrow keys. Some menus require that the user proceed sequentially through all possible options before being allowed to exit the window. This is a minor aggravation when only one or two options need to be reset. Overall, the interface is reasonably intuitive and the average user would learn to navigate through the menus with minimal effort.

\section{Using the Program:}

The input data must adhere to GEOEAS file format, but this format is straightforward and allows the sample data matrix to be in "free format" (space delimited is acceptable). Two of the GEOEAS modules allow the user to create or modify a data file (module TRANS) and to compute basic univariate statistics for the data set (module STATI).

$P R E V A R$ is a "preprocessor" program which generates pair comparison files (.pcf files) required by the program VARIO. PREVAR sets the number of lags and lag boundaries and stores the results. The user can specify $\min / \mathrm{max}$ limits on the interpoint distances used to build the pair comparison file. The default is to use all possible pairs as long as the maximum number of allowable pairs (16,384 pairs) is not exceeded.

The VARIO tool computes and displays the results from the previously stored $p c f$ file produced by program $P R E V A R$. The user may view a variogram plot and manually fit the variogram to the displayed data. Options include:

- Tolerances may be set so that only a subset (the limits of which are defined by the user) of the lag results stored in the pair comparison file are used in the VARIO tool

- Variogram plots, boxplots, scatter plots, and histograms may be viewed.

- The results (average distance, variance, variogram and other statistics) for individual lags may be examined and the lag spacing may be redefined if desired

- Intermediate results may be saved in a Geo-EAS data file for analysis.

The first window allows the user to specify the name of the desired $p c f$ file and to set the range (minimum and maximum) of the $Z$-values to be used in the variogram computation. This window also provides some summary statistics for the paircomparison file. The subsequent window (OPTIONS) allows the user to specify the kriging direction (the default is omnidirectional) and the lag spacing. The default 
number of lags is 20 covering the entire range of the data (so that lag increment $=\max$ interpoint distance / 20). The OPTIONS window displays the number of lags and the lag breakpoints for all lags. The user can respecify the lag spacing, the breakpoints for individual lags, and the maximum interpoint distance.

After specifying all options and selecting EXECUTE from the VARIO menu, the Variogram RESULTS window appears, displaying the number of pairs in each lag, the average distance within the lag and the estimated gamma for each lag. From here, the user may select various options to view the data specific to each lag or to view a plot of the binned empirical variogram. Selecting the Model option allows the user to specify a variogram model. Available models are spherical, gaussian, exponential, and linear. No automatic model fitting is provided. The user must manually assign a sill, range and nugget based on inspection of the variogram plot. Note that in GeoEas the "sill" refers to the "partial sill" or the "true" sill minus the nugget. The resulting line may then be plotted over the binned empirical variogram. Once a satisfactory model fit is achieved, the user can save those values and exit the VARIO module.

The KRIGE module is used to predict a 2D grid of values for 1 or more variables.

- Program parameters may be saved in parameter files for later use.

- A grid of up to $100 \times 100$ points may be kriged.

- Up to 10 variables may be kriged for each point or block in the grid.

- Debug options provide views of the search area and the kriging system.

- The kriging area may be restricted by using a polygon boundary file.

- The estimates (and kriging stddevs) are stored in a grid (.GRD) file.

- A contour map may be produced from the grid file using CONREC.

The kriging options window allows the user to specify the kriging type, either simple or ordinary. Simple kriging assumes that the true mean of the field is known. Since this is rarely the case, ordinary kriging is normally used. Other kriging options are point vs. block kriging, grid size and grid origin (default is $10 \times 10$ with origin equal to the minimum $\mathrm{X}$-value by the minimum $\mathrm{Y}$-value). The grid is roughly partitioned equally over the total data range. 
Example of GEOEAS gridding defaults:

\begin{tabular}{|lcc|}
\hline \multicolumn{2}{l}{ Grid Parameters: } & \\
& $\mathrm{X}$ & $\mathrm{Y}$ \\
Variable: & Easting & Northing \\
Origin: & 569.211 & 559.295 \\
Spacing: & 19.035 & 18.734 \\
Number: & 10 & 10 \\
& & \\
\hline
\end{tabular}

The search parameters set in the KRIGE OPTIONS window are important for the simulations. The kriging calculation can be performed using all points within the specified search radius or, alternatively, the search can be partitioned into 4 or 8 regions ("sectors"). If the latter option is chosen, the search quadrants or octants are defined by $90^{\circ}$ or $45^{\circ}$ arcs, respectively, centered around the sample point. Separate searches are performed for each sector and the kriging is then conducted by combining the points from each sector search. This approach assures that some neighbors are selected from each sector rather than, for example, having all neighbors drawn from one sector around the grid node. The default number of sectors is 1 , with a minimum number of points $=8$ (lowest acceptable value $=1)$ and maximum $=16$ (maximum acceptable value $=24$ ). The default search radius is $1 / 4$ the maximum easting or northing distance (whichever is greater).

From the OPTIONS window, the user can select the Variables/Models option to specify a variogram model (presumably the model defined in the VARIO module) to be used for kriging. The user then returns to the KRIGE OPTIONS window to perform the kriging. The results are displayed in a plot and stored in a grd file.

General comments on program:

GEOEAS is a powerful well-designed geostatistical software package. It includes all of the essential spatial routines and has an impressive set of options for customizing the procedures. However, the interface is primitive by today's standards and is likely to be frustrating to a user accustomed to a multi-window interface and mouse support. 


\title{
B. GEOPACK:
}

\author{
Developers: $\quad$ S.R. Yates \\ USDA/ARS- U.S. Salinity Laboratory \\ Riverside, California \\ M.V. Yates \\ University of California \\ Riverside, California \\ Distributed by: \\ International Ground Water Modeling Center \\ Colorado School of Mines \\ Golden, Colorado 80401-1887 \\ http://www.mines.edu/igwmc/ \\ Center for Subsurface Modeling Support (CsMoS) \\ http://www.epa.gov/ada/models.html/ \\ U.S Salinity Laboratory \\ http://www.ussl.ars.usda.gov/
}

Distribution terms: public domain freeware

General Notes:

GEOPACK consists of a package of programs for conducting analyses of the spatial variability of one or more random functions. GEOPACK includes several statistical analysis options. Basic statistics which can be calculated include the mean, median, variance, standard deviation, skew, kurtosis, maximum value, and minimum value. Programs are also included for linear regression, polynomial regression, and the Kolomogorov-Smirnov test for distribution, and for calculating various percentiles of a selected data set. Variogram programs allow determination of a sample semivariogram, the cross-semi-variogram, or a semi-variogram for combined random functions for a two-dimensional spatially-dependent random function. GEOPACK also can calculate ordinary kriging and co-kriging estimators in two dimensions and their associated estimation variance.

Various graphics capabilities are included in GEOPACK such as linear or logarithmic line plots of the input data, contour plots, and block diagrams. The GEOPACK graphics programs produce intermediate quality output and are intended for quick and easy 
graphic illustrations. GEOPACK allows the incorporation of other geostatistical programs, such as GEO-EAS, so unique features of these programs can be accessed from within GEOPACK.

\section{Limitations of the Package:}

- The package suffers from assorted problems apparently associated with DOS memory limits; certain configuration changes must be made to the system files (Autoexec.bat and Config.sys) in order for all features of the program to be operational. The necessary changes are described in the software documentation.

- Interface is awkward: user can not step backward through the menus

- Some default behaviors are counter-intuitive (e.g., grid origin automatically set to $0.0,0.0$ regardless of data set coordinates)

- The input file requires strict fixed record/fixed field formatting. Free-form formatting (space or tab delimited) is not allowed

Strong features of the package:

- Ability to predict both grids and arbitrarily located points

- Ability to fit a variogram model automatically and then modify the model by eye if desired

User Interface:

As with GEOEAS, the DOS-based interface of this program will seem awkward to users accustomed to more recent Windows software: GEOPACK also suffers from a number of peculiar glitches that may plague the unsuspecting user. Some procedures required a trial-and-error approach to eventually coerce successful execution. The graphics are acceptable for screen display, but would not be suitable for publication or presentation.

\section{Using the Program:}

The program includes 5 basic modules:

- Data Set Utilities Menu

- Statistics Menu

- Variogram Menu

- Ordinary [Co]Kriging Menu

- Disjunctive [Co]Kriging Menu

The user must first select a data set in the data set utilities menu. The data format is highly specific and failure to properly specify the data fields results in a variety of errors. When the program reads in the data file, the user may be prompted for a Cutoff value: this is the upper cutoff value for the $\mathrm{Z}$-values (no minimum value is provided). 
Data points exceeding the cutoff value are discarded. If the program fails to display a screen indicating the number of available data points successfully read, an error has occurred in reading the data. The user should examine the data file for possible errors in the format specification.

After specifying a data set, the Variogram Calculation (Sample) menu allows the user to specify the various parameters and options for the variogram computation. (Note that the user must complete this sample variogram and edit the variogram file before using the Automatic Model Fit tool). The variogram parameters are assigned sequentially through a series of new screen windows, with the defaults displayed in the lower portion of each window. (Alternatively, the user can select Set Program Parameters to display a window where most of the required variogram parameters can be set).

The default number of lags (NLAG) is 12. The default lag increment (BLAG) is defined in the help files as follows:

$B L A G$ is the basic distance separating the lagged intervals. Generally, $B L A G$ should be in the range:

$$
B L A G \sim 1 / 2 \text { (Maximum Field Length)/(Number of Lags) }
$$

where $N L A G$ is the number of lags. Ideally, the length $B L A G * N L A G$ should be no more than 50 to $70 \%$ of the maximum length of the region of interest.

The GEOPACK source code sets the defaults for BLAG as:

$$
\text { BLAG }=\left(\operatorname{SQRT}\left(\mathrm{dx}^{2}+\mathrm{dy}^{2}\right)\right) /(2 * \mathrm{NLAG})
$$

where $\mathrm{dx}$ and dy are equal to the (maximum value - the minimum value) along that axis.

Once all variogram parameters have been specified, the Variogram Calculation window is displayed with the average distance and variogram estimate for each lag.

After generating a variogram file, the user can proceed to the Automatic Model Fit procedure. Available models include spherical, gaussian, linear, exponential, and power models. A non-linear least squares analysis is performed and the estimated parameter values for the sill, range and nugget are displayed. A plot of the variogram is also displayed with the fitted model. It should be noted that if an exponential model is 
selected, the variogram model is parameterized differently than for the other models.

Exponential semivariogram model used by Geopack, Geoeas, and S-Plus:

$$
\Gamma=s\left(1-e^{\frac{-d}{r}}\right)
$$

Exponential semivariogram model often presented in the geostatistical literature:

$$
\Gamma=s\left(1-e^{\frac{-3 d}{r}}\right)
$$

It is evident from the above equations that parameterizing the exponential model according to the first formula (as in Geopack, Geoeas and S-Plus) results in a "range" parameter that is $1 / 3$ of the value in the second formula. Also, using the same parameterization, the "range" does not correspond to the distance at which the variogram plateaus at the sill. The user should be attentive to this fact when specifying the variogram parameters.

After fitting a model automatically, the user may adjust the parameters in the Manual Model Fit module. Once a satisfactory model is achieved (via either the automatic or manual options), the model parameters must be saved in a file by using the Edit Variogram Model File option before kriging can be performed.

Within the Ordinary [Co]kriging module, the user can select among various options for the graphical output, select point or block kriging, choose the number of nearest neighbors (default $=10$ ), select the maximum search radius (default $=0.0$ ), specify anisotropy (and angle) and specify the grid size and origin.

Two of the GEOPACK kriging defaults are potentially problematic or confusing. The default search radius.(RMAX) is 0.0 . Although this default appears to suggest that the searching distance $=0$ units (so that no neighbors will be found), Geopack actually sets RMAX = the range of the semivariogram if the default is used. The help files provide the following information relating to RMAX:

The maximum allowed radius, RMAX, specifies the search area for the data that will be used in selecting the data for the estimation. Only the data points located within a radial distance of $R M A X$ will be used. 
If a large $R M A X$ is selected, then, in general, the maximum nearest neighbors (i.e., NZ, this is specified elsewhere) will be used in the estimation process. If a small RMAX is selected then it is possible that no points or one point will be used.

$R M A X$ should be some value between $(1 / 4$ to $1 / 2$ range $<R M A X<$ range) where range is the range of the variogram.

For the simulations, a variety of search radii (including the range and $1 / 2$ the range) were used to reproduce the default behaviors of the software packages under consideration.

Another difficulty with the Geopack defaults is that the default origin for the kriging grid is $(0.0 \times 0.0)$. This may place the grid origin at some point far removed from the data coordinates. The user must manually identify an appropriate point for the grid origin (for example, the minimum $\mathrm{X}$ by minimum $\mathrm{Y}$ value) to position the grid as desired. Although the user may specify a grid of up to 100 by 100 points, attempting to krige on a grid larger than 50 by 50 apparently resulted in memory overloads and incomplete results.

After specifying the desired grid, the kriging results are displayed. These results may also be written to a data file if the user previously selected this option.7 


\section{Surfer}

Developers: $\quad$ Golden Software

$80914^{\text {th }}$ Street

Golden, Colorado 80401-1866

800/ $972-1021$

Distributed by: $\quad$ same

Distribution terms: $\quad$ free demo including most features;

Full version $\$ 499.00$

\section{General Notes:}

Surfer is a grid based contouring package that transforms $\mathrm{X}, \mathrm{Y}, \mathrm{Z}$ data into a grid file which may then be used to produce contour and surface maps. A wide assortment of mapping features is available and the package can produce sophisticated, colorenhanced maps. Although the input data can be randomly dispersed over an area, Surfer's gridding routine must interpolate the data onto a grid before it can be displayed as a map. Map styles include contour, surface, and shaded relief maps with 3D representation of the Z-axis. The input data can be "posted" so that it is superimposed onto the maps. Map colors, text and other features are highly customizable.

\section{Strong features of the package:}

The promotional literature for Surfer lists the following features:

- Interpolate from an unlimited number of $\mathrm{XYZ}$ data points

- Produce grids of virtually any size

- Choose from Inverse Distance, Kriging, Minimum Curvature, Polynomial Regression, Triangulation, Shepard's Method, Radial Basis Functions for the gridding operation

- Specify isotropic or anisotropic weighting

- Full control over the grid line geometry including grid limits, grid spacing and number of grid lines

- Choose from different search methods including simple, quadrant, octant and all points

- Specify search ellipses at any orientation and scaling

- Spline or matrix smoothing to smooth the grid file

- Grid math to perform mathematic operations between grid files 


\section{Limitations of the package:}

While Surfer is feature-rich compared to the other packages discussed herein, preliminary examination revealed several shortcomings:

- Kriging on random point locations is not allowed; all kriging is done on a grid system. However, Surfer provides a work-around to this limitation through the Residuals command (discussed further below)

- No variogram plots are produced so that the user must refer to other software to examine the variogram fit and, if desired, adjust the default values for the sill, range and nugget

- The maps generated by Surfer will generally be larger than the area encompassed by the input data. The only way to generate a map specific to the data input region is to specify grid options that incorporate only that area. This may be difficult to do if the input data and/or the region of interest are not oriented parallel to the major axes.

- Surfer does not have the capability to produce contour plots or 3D surface plots directly from an XYZ data input file. The mapping data requires that the input data be a Surfer-generated binary file.

- The kriging routine does not report standard errors for the estimates

\section{User Interface:}

This package provides a state-of-the-art user interface including multiple windows, pop-up menus, full mouse control, an Excel-style worksheet for creating or editing data files and many other common Windows features. This is a decided advantage over the DOS programs reviewed above. Right-clicking on specific features of the maps invokes pop-up menus for customizing features specific to that map item.

\section{Using the Program:}

The input data may be a delimited ascii text file (extension .dat), a Lotus file (.wkx) or an Excel worksheet file (.xls). Optionally, the data may be imported into Surfer's worksheet and manipulated as desired. An assortment of intrinsic functions facilitates data handling.

Once the XYZ data file has been created, the user can select the Data option from the Grid menu to specify a grid that defines the kriging locations. This invokes the "Scattered Data Interpolation" window, in which data fields are specified, the grid geometry is defined, and the desired interpolation method is selected. A number of interpolation methods are available in addition to the kriging routine. Available variogram models include linear (the default) exponential, gaussian, hole effect, quadratic, rational quadratic, and spherical. Oddly, the default values for the sill, range and nugget do not change if the user selects a variogram model other than the default 
linear model. If the number of sample points is less than 200 , the default search algorithm uses all points for kriging the grid. Alternatively, the user may override this default and specify the search radius, minimum number of neighbors and maximum number of neighbors for kriging.

The search for kriging neighbors may be subdivided into quadrants or octants ( 4 or 8 sectors) around the grid node. If multiple sectors are used, separate searches are conducted within each sector and the kriging is then conducted by combining the points from each sector search. This approach assures that some neighbors are selected from each sector rather than, for example, having all neighbors drawn from the "northeast" sector around the grid node. After all options are set, Surfer generates a .grd file containing the kriged estimates for all grid nodes. The default file type is binary, but the kriging results may also be saved in a standard $\mathrm{XYZ}$ ascii format. Surfer does not report, and there is apparently no way to retrieve, the standard errors for the kriging estimates:

As previously noted, the kriging procedures in Surfer can only be executed on a grid system. In order to produce kriging estimates for arbitrary locations the user must take advantage of the Residuals command. This command prompts the user to specify the name of an XYZ data file with the locations of the arbitrary points. Surfer will then use a linear interpolation method to estimate the value of $Z$ at each point, based on the kriging predictions at the surrounding grid nodes. Rather than report the interpolated value directly, Surfer outputs the residuals between the observed value (given in the $\mathrm{XYZ}$ data file) and the interpolated value. If the XYZ data file contains all 0's in the Zposition, then the reported values are equal to the negative of the interpolated values. This provides a roundabout way to obtain kriging estimates at any arbitrary location that does not fall on a grid node.

The mapping options require a binary .grd file for input. The default settings for the contour, surface and shaded relief plots are acceptable, but the maps may be enhanced using the many available graphics options. As noted above, one potentially confusing aspect of the resultant maps is that, depending on the grid geometry specified by the user, the map may extend well outside the input data region. Extrapolating beyond the data area by kriging can produce extremely eccentric surfaces (that can be very misleading). If this was not the intended effect, the user may need to respecify different settings for the grid. This may require rotating the $X-Y$ axes, a facility not included in Surfer.

One of the most impressive features of Surfer (and one not available in either of the DOS packages discussed above) is the ability to write macro scripts to automate tasks. Surfer includes a scripting language, GS Script, which is very similar to BASIC. A 
number of standard math, string manipulation, and flow control functions are available for writing Surfer programs. A large set of macro commands specific to Surfer are also included to enable the automation of tasks normally executed from the menus. The macro structure is highly intuitive, and we were quickly able to write a simple (12 line) program to iteratively select a series of data files, generate kriged estimates for a grid and store the results in an ascii file. Surfer also supports OLE Automation, so that Surfer macro commands may be linked with other OLE 2.0 compatible programs such as Visual Basic. This capability enables Surfer to be incorporated into a more complex programming context requiring multiple languages and/or applications.

The kriging algorithms incorporated into Surfer are not fully described in either the hard cover or on-line documentation. (The manual simply refers the reader to the geostatistical literature for a more thorough explanation of kriging). It was not apparent to the investigators how the default values for the sill and range were generated. The investigators have contacted the developer of the kriging routines in Surfer to acquire additional information required to correctly simulate Surfer's default behaviors. However, it appears that the kriging routines in Surfer, like S-Plus, were very similar to the algorithms in GSLIB. Resetting S-Plus' variogram parameters to accord with the Surfer defaults (and using Surfers grid nodes as the kriging points in S-Plus) caused the two programs to report precisely the same kriging results. 


\section{S-Plus}

Distributed by: MathSoft

Data Analysis Products Division

1700 Westlake Avenue North

Suite 500

Seattle, Washington 98109

(206) 283-8802

(800) 569-0123

website: http://www.mathsoft.com

Distribution terms: commercial product

General Notes:

S-Plus is a command driven object-oriented programming language with a number of built in functions that allow for a variety of statistical analyses. The package is more flexible than the others discussed in this report, but the flexibility comes with a price tag. Users need to become proficient in the S-Plus language and they need more than a passing familiarity with the underlying statistical theory because there is a greater opportunity for getting into trouble. The package is also the most expensive of the 4 reviewed here. The basic package for a Windows version of S-Plus is over $\$ 1000.00$ and the SpatialStats module is another $\$ 600.00$ or so. Academic discounts are available. A user who is primarily interested in doing only spatial analysis will pay for a number of other capabilities that may be of only limited usefulness or interest.

User Interface:

S-Plus is available for a number of different platforms. The version discussed herein is the Windows PC version. However, all of the different platform versions have a similar interface. Although some pull-down menus are provided, the program is primarily command-driven. The user must be familiar with S-Plus data handling and know how to invoke the program's intrinsic functions.

Using the Program:

There are 3 basic functions that are used for geostatistical analysis. These are:

- variogram

- krige

- predict.krige

Each of these is summarized below. 
The S-Plus function variogram computes an empirical (or sample) variogram for twodimensional data. The function assumes that the data is stored in an S-Plus object called a data frame. A data frame is essentially an array whose columns contain information on variables of interest and whose rows represent different observations. The simplest spatial data set would be in the form of a data frame with, say, columns 1 and 2 containing spatial coordinates and column 3 containing the value of the variable of interest. For illustrative purposes we will assume that we have the Bliss Farms dioxin data in a data frame called bliss.dat with easting and northing coordinates in columns 1 and 2 and the logged dioxin values in column 3 . Assume that these columns have names FE, FN, and CONC respectively. One could compute an omindirectional variogram by issuing the command:

$$
\text { bliss.var_variogram(CONC } \operatorname{loc}(\mathrm{FE}, \mathrm{FN}) \text {,data=bliss.dat) }
$$

The resulting variogram would be stored in the S-Plus object bliss.var.

There are a number of default options that can be modified as needed. The 3 most important are maxdist, nlag, and minpairs. Maxdist is the maximum distance to include in the variogram computations. Pairs of data separated by more than maxdist are not included. The default value for maxdist is the maximum Euclidean distance across the spatial field divided by 2 . The default number of lags (nlag) equals 20 . The default value for the minimum number of pairs (minpairs) equals 6 . If we wanted to compute a variogram with maxdist $=150$, nlag $=12$, and minpairs $=2$ then the call to the function variogram would be:

bliss.var_variogram(CONC $\operatorname{loc}(\mathrm{FE}, \mathrm{FN})$,data=bliss.dat,maxdist=150,nlag=12, minpairs $=2$ )

The manner in which S-Plus chooses lag width and pairs to include in a given lag are identical to that used in GEOPACK, although the GEOPACK default values for maxdist, nlag, and minpairs differ.

Once the empirical variogram has been returned the output can be used to fit a valid variogram function. S-Plus has two methods of fitting such a function - by eye and by nonlinear least squares. The intrinsic function model.variogam allows one to interactively fit a variogram by eye to the empirical variogram, but the documentation suggests that the fitting be done automatically. The S-Plus function that performs the best for this purpose is called nlmin. This is one of those areas where a familiarity with the subject area is critical. The function nlmin is not easy to use unless one understands the mechanics of nonlinear least squares fitting. Proper use requires specification of an objective function that is to be minimized. Further constraints may need to be imposed 
on the objective function to insure that parameter estimates are valid. There are 4 variograms provided by S-Plus, the spherical, the exponential, the gaussian, and the power models. A fifth model, the linear variogram, is just one version of the power model. The details of using $n$ lmin are not presented here.

Once valid estimates of the chosen variogram model have been obtained the S-Plus function krige can be used to krige the field. This function requires a formula specifying which type of kriging is to be done, and the specification of the covariance structure given by the variogram model and parameter estimates. Assuming that a spherical model has been fit to the empirical variogram with parameter estimates of range $=60$, sill $=3$, and nugget $=0$, kriging results can be generated by the following call:

bliss.krige_krige(CONC $\sim \mathrm{loc}(\mathrm{FE}, \mathrm{FN})$,data=bliss.dat,covfun=spher.cov, ränge $=60$, sill $=3$, nugget $=0$ )

Unlike the other packages S-Plus has no facility for user control of the search radius or the maximum/minimum number of nearest neighbors to include in generating the kriging equations. Further, S-Plus documentation contains no information on just how the program automates the parameter assignment. We suspect that S-Plus is simply using all neighbors in the field. We will attempt to verify this with the S-Plus technical support.

Predictions can now be generated using the S-Plus function:

$$
\text { bliss.predict <- predict.krige(bliss.krige) }
$$

The S-Plus object bliss.predict contains predictions and prediction standard errors over a default 30 by 30 grid. S-Plus also allows for predictions at any specified location. Thus, if there were a set of 100 spatial locations at which one wanted predictions stored in an S-Plus object called new.dat then predictions at those locations can be generated by the following call:

$$
\text { bliss.predict <- predict(bliss.krige,newdata=new.dat) }
$$

The S-Plus object bliss.predict now contains predictions and standard errors for the 100 specified spatial locations in the object new.dat.

Summary of S-Plus Capabilities:

S-Plus has the most power and flexibility of the packages discussed here. S-Plus has graphical capabilities that rival those of Surfer but comparable graphs would not be 
easy to produce. S-Plus also has significant capabilities for exploratory data analysis, capabilities far superior to those of the other packages. The only real down side to SPlus is the difficulty of using it. It is not a user-friendly package, but it was not really designed to be. A recent release of S-Plus (Version 4, released September 1997) goes a long way toward rectifying the problems inherent in the S-Plus' traditional commanddriven interface. The new version conforms to most of the standards for Windows applications, with pull-down menus and full mouse control for accessing common procedures. It also includes an Excel-style spreadsheet for entering and/or editing data. At the time of report preparation (October 1997), the S-Plus' spatial statistics module had not been recompiled for Version 4 but it is expected to be released in early 1998. 


\title{
E. GS+ Windows (3.03.40 Beta Evaluation Version)
}

Distributed by:

\author{
Gamma Design Software \\ PO Box 201 \\ Plainwell, Michigan 49080
}

(616) 685-9011

email: support@GammaDesign.com

web address: $\underline{\text { http://www.gammadesign.com }}$

Distribution terms: $\quad$ commercial product (free trial version available)

General Notes:

GS+ is a full-featured geostatistical analysis program for Windows. It analyzes spatial data for autocorrelation, performs kriging, and produces high-quality 2D and 3D maps. The autocorrelation options include semivariance, Moran's I analysis, and fractal analysis. Both block and point kriging may be performed, with point kriging available for either gridded or arbitrary coordinate locations.

Although the beta version used for this evaluation included several minor glitches, it was apparent that GS+ has distinct advantages over any of the other packages we examined. It combines many of the best features available in other software into a single, intuitive interface and includes a number of additional options not available in the other packages. For example, it is the only Windows program that performs both variogram modeling and kriging using menu and mouse-driven options. The variogram modeling is especially powerful in that each time a semivariance analysis is performed, the program fits five separate models (spherical, exponential, gaussian, linear, and linear to sill) to the data and displays diagnostic statistics to evaluate the fit of each model. The "best" isotropic model is automatically plotted and the user is allowed to adjust the model parameters or to select a different model if desired.

\section{Limitations of the Package:}

We encountered only three features missing from this program which would be useful for refining the kriging analysis:

- There are no options to set the search type to perform a "sector" type search (using quadrants or octants) as was available in Surfer, GeoEas, and GeoPack

- There is no way to set the minimum number of neighbors used for kriging and it is not clear what default number is used or how the program handles cases with insufficient neighbors 
- The program does not incorporate any sort of scripting language to enable automation of more complex or repetitive tasks

- The worksheet (data entry) window is adequate for most simple tasks, but does not allow complicated calculations using intrinsic functions as does the Surfer worksheet

Strong Features of the Package:

- High quality graphical output (2D, 3D and "transect" maps with rotation, customization and posting of data points)

- Best variogram modeling.routine of any package, including isotropic or anisotropic models, automatic fitting of five model types, and easy facility to adjust model parameters

- Three types of autocorrelation analysis: Semivariance, Moran's I and fractal analysis

- Performs both block and punctual kriging

User Interface:

The user interface of GS+ is relatively intuitive, although options are not always as obvious as those of the other Windows program, Surfer. There are basically five windows which correspond to different aspects of the spatial analysis: Data Worksheet, Data Summary, Semivariance Analysis, Kriging Analysis, and Mapping. In typical Windows style, the options for each analysis are selected by a combination of mouse clicks, radio buttons, and pop-up windows. Help topics are available for most procedures.

\section{Using the Program:}

Analysis begins by opening a file into the worksheet window. The user can import data files in several formats (GS+, GeoEas, Surfer XYZ or Custom). Text files can be delimited with space, tab or commas. After importing or entering new data, the columns containing the $\mathrm{X}, \mathrm{Y}$ and $\mathrm{Z}$ data are specified and the Recalculate options is used to preprocess the data for semivariance analysis.

Although three types of autocorrelation analysis are available, only the semivariance analysis window is discussed below (the other two autocorrelation procedures have similar interfaces). The Semivariance window allows the user to specify the lag distance (maximum distance for which lags are computed; equivalent to variable Rmax in other programs) and the lag interval. Alternatively, the user can designate nonuniform lags. The default for Rmax appears to be $80 \%$ of the maximum interpoint distance, divided into 10 equal lags. A total of ten separate variogram models are automatically calculated: five isotropic models and five anisotropic models (spherical, 
exponential, gaussian, linear, and linear to sill). For the anisotropic models, four plots are displayed corresponding to $22.5^{\circ}$ offsets.

Each of the isotropic models is accompanied by diagnostic statistics to help the user compare the relative fit to the data. These diagnostic statistics are: partial sill / true sill, $\mathrm{R}^{2}$, and RSS. (The significance of the first of these diagnostics is not clear). One of the five isotropic models is identified by the program as the "best" model and is the one that will be used for kriging unless the user overrides this selection. The criteria for identifying this model appears to be whichever of the spherical, exponential or linear models has the greatest $R^{2}$. The user may optionally edit the variogram model parameters and preserve those settings for the subsequent kriging analysis.

After obtaining a satisfactory variogram model, the data are ready for kriging. The kriging window allows the user to verify or set the kriging options. These options include the kriging locations (grid specification or arbitrary coordinates read from a file), and the search specifications (default $=$ maximum of 16 neighbors with no option to set the minimum number of neighbors). The default search radius is equal to the maximum interpoint distance.

There are also several potential trouble spots in the kriging window that the unwary user should be attentive to. First, the interpolation method (block or point kriging) must agree with the suffix of the output file: *.blk for block kriging and *.pun for point kriging. Changing either of these settings will toggle the other. Second, the user should verify that the Variogram Model Type displayed in this window is correct: occasionally the program seems to switch between isotropic and anisotropic models unexpectedly.

The GS+ kriging options include two facilities not available in other programs we examined. The Cross-Validation model enables a jack-knifed analysis to predict the Zvalue at each of the input coordinates. This feature is useful to evaluate alternative models for kriging. Another useful feature is the ability to restrict kriging to irregular shaped areas by defining polygons within which kriging is either performed or excluded. Any number of vertices may be used to define these areas. This feature would help to avoid the eccentric surfaces we sometimes found with other programs when the data coordinates were oriented obliquely to the main axis and the kriging grid (built using $\mathrm{min} / \mathrm{max}$ of the $\mathrm{X}-\mathrm{Y}$ coordinates) extended far outside the region of the data.

After kriging, a map is automatically produced to display the kriging results. This map uses the kriging output file (either a *.blk or *.pun format) as input. Among the display options are posting of the input data, map type (contour, surface, wireframe), 
smoothing and number of contours. The resultant map may be rotated and the scales, titles, and axes may be edited. The maps are of acceptable quality for either printing or export (as wmf, emf, or bmp formats), although we encountered some difficulty when copying GS+ maps to the clipboard for display in another application.

\section{General Comments on the Program:}

Of all the geostatistical packages we evaluated, GS+ offered the most impressive suite of features. The inclusions of both interactive variogram modeling and kriging in a single package is the major advantage of GS+ over its other Windows competitor, Surfer. Surfer's mapping capabilities remain the best of the group, but the maps displayed by GS+ are more than adequate for most applications. The developers of Surfer intend to include a variogram modeling feature in the next release, but without this functionality the user is required to resort to some other program (e.g., VarioWin) to model variograms prior to kriging. 


\section{COMPARING KRIGING RESULTS USING S-PLUS AND FORTRAN SIMULATIONS}

This portion of the report provides a preliminary overview of the methodology used to simulate the behavior of the kriging software packages discussed above and to compare the relative performance associated with the different kriging protocols. The results from these simulations are presented in a separate report: Overview of Kriging Simulation Results (Harting and Cherry: 10/27/97).

The strategy used for comparing kriging results required that the default settings for the kriging routines be simulated in an external programming language. This strategy was necessary because GEOEAS and GEOPACK in particular do not lend themselves to simulation studies. The intent of this approach is to emulate as closely as possible the results that might be produced by the average practitioner, perhaps only moderately familiar with kriging theory. While each program is represented by one set of defaults, additional simulations were run using various combinations of default settings not directly associated with any particular package. This approach was helpful in determining the sensitivity of the kriging results to each of the input parameters.

Default behaviors/settings of the kriging software packages were determined by software documentation, program execution, or (in the case of GEOEAS and GEOPACK) directly from the Fortran source code. As previously noted, in some cases no defaults were provided and the user was required to specify a value. In these cases, a reasonable range of values was substituted for the defaults.

The data set used for the simulations was the Bliss Farm data set previously described by Blacker and Goodman (Environmental Science and Technology). The data set consists of dioxin concentration measurements at 400 locations.

Simulations were carried out by repeatedly subsampling ( 500 times) 50 locations from the 400 available in the data set and predicting logged dioxin concentrations at the 350 remaining locations. An empirical variogram was computed for each of the 500 sample data sets. We wrote S-Plus functions to emulate empirical variogram computation in GEOEAS and GEOPACK and we fit spherical variograms to the empirical variograms using a nonlinear least squares fitting routine available in S-Plus. Kriging results were determined using Fortran code we wrote (for GEOEAS or GEOPACK) or in S-Plus (for S-Plus). Code for simulating Surfer's kriging routines was deferred until additional information could be obtained from the software's developers. We carried out a series of simulations using different default settings corresponding to those available in the various packages. 
Various summary statistics were cumulated for each default setting and each location. The summary statistics of interest were the following:

1) mean squared residual by default type and by each of the 400 locations in the data set

2) percent of the estimates for which the $90 \%$ confidence interval correctly captured the actual measurement ("coverage")

3) width of the $90 \%$ confidence interval (constructed from the standard error of the kriging estimate)

For a second round of simulations, the logged dioxin concentrations were detrended using either a linear or quadratic model. The kriging was then conducted on the residuals from the detrending. A final estimate was derived by adding the krige estimate to the trend estimate for each location. 
Table 1: Summary of the major features of the geostatistical software packages examined for the kriging comparison project.

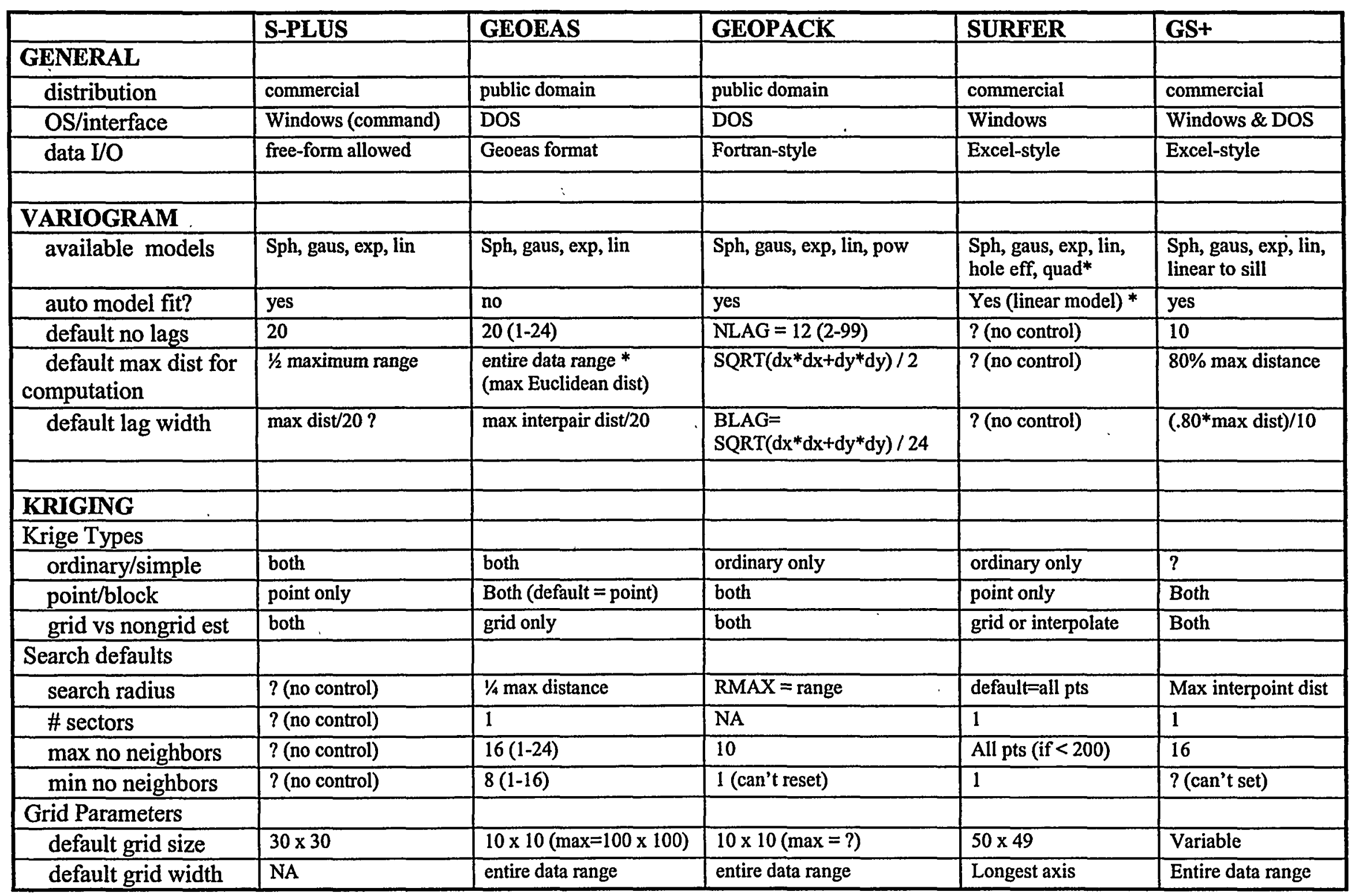

* Surfer provides no variogram modeling facility. It provides default parameters for a linear variogram that are used for kriging or the user can specify other models \& parameters 


\section{HOW THE SOFTWARE COMPARISON MAPS WERE CREATED}

We have a series of maps that show contrast in the kriging results from Surfer, Geopack and GS+. These three programs were chosen because they are the only ones which fully automate the kriging process by not only calculating the empirical semivariogram, but also providing routines for automatically fitting a model to this semivariogram.

In Surfer and GS+, the type of model is specified by the program (always a linear model in Surfer; best-fitting model in GS+), while in Geopack the user must specify which type of model to fit to the data.

\section{Iteration \#1: First Efforts at Comparing Software with Wag-1 Data}

Our earlier version of this comparison was biased in several respects. We intervened in Geopack to adjust the model to our liking. Our justification in so doing was that the empirical semivariogram showed a continuous decline in the outer lags and a variogram model fit to these lags would not be appropriate for defining the true correlation structure of the data. We therefore trimmed the maximum distance for variogram computation from the default (equivalent to $1 / 2$ the data range) down to 10 lags of width 0.05 (in revised coordinate space). We then fit an exponential model to this truncated empirical semivariogram: partial range $=.027841$ (true range $=.083523$ ), sill $=5.3865$ and nugget $=0.0$. (In the original coordinate space the partial and true ranges are 27.841 and 83.523 , respectively).

If we had also left the kriging search radius at its default (equal to the true range of the variogram), we would have had a preponderance of kriging failures. Instead we set the search radius approximately equal to the maximum interpoint distance (3.0: revised coordinates). After kriging on a 50 by 50 grid we produced the plot originally used to depict the differences in Surfer vs. Geopack.

\section{Iteration \#2: Revisiting the Software Comparison Issue}

Recognizing that the earlier version was not a valid indication of how the different software packages handled the Wag- 1 data, we completed a second series of runs. The actual defaults from each program are given in the table below.

Table 1: Comparison of kriging software defaults using the revised coordinate system

\begin{tabular}{|l|l|l|l|}
\hline & Surfer & GS + & Geopack \\
\hline $\begin{array}{l}\text { Number of } \\
\text { lags }\end{array}$ & NA & 9 & 12 \\
\hline Lag Width & NA & 0.2411 & 0.14 \\
\hline Total dist & NA & $2.411(80 \% \max$ interpt & $1.68(\sim 1 / 2$ field \\
\hline
\end{tabular}




\begin{tabular}{|l|l|l|l|}
\hline & & dist) & width) \\
\hline Type of model & linear & spherical & exponential * \\
\hline True Range & "length" $=1.66$ & 0.146 & $\begin{array}{l}.014305^{*} 3= \\
0.042915\end{array}$ \\
\hline Sill & "scale" $=4.31$ & 4.030 & 3.0068 \\
\hline Nugget & 0.0 & 0.390 & 1.4357 \\
\hline Search Type & quadrant & simple & simple \\
\hline $\begin{array}{l}\text { Number } \\
\text { neighbors }\end{array}$ & $6 /$ sector (min 5 total) & $?-16$ & $1-10$ \\
\hline Search radius & $1.66(1 / 2$ field width) & 3.013 (max interpt dist) & 0.043 (true range) \\
\hline
\end{tabular}

* Geopack is not able to fit a spherical model to these data

Table 2: Comparison of kriging software defaults using the original coordinate system

\begin{tabular}{|l|l|l|l|}
\hline & Surfer & GS & Geopack \\
\hline Number of lags & NA & 9 & 12 \\
\hline Lag Width & NA & 241 & 138.40 \\
\hline Total dist & NA & $\begin{array}{l}2411(80 \% \text { max } \\
\text { interpt dist })\end{array}$ & $\begin{array}{l}1660(\sim 1 / 2 \text { field } \\
\text { width })\end{array}$ \\
\hline Type of model & linear & spherical & exponential * \\
\hline True Range & "length" $=1660$ & 146 & 42.915 \\
\hline Sill & "scale" $=4.31$ & 4.030 & 3.0068 \\
\hline Nugget & 0.0 & 0.390 & 1.4357 \\
\hline Search Type & quadrant & simple & simple \\
\hline Number neighbors & $\begin{array}{l}\text { 6/sector (min 5 } \\
\text { total) }\end{array}$ & $?-16$ & $1-10$ \\
\hline Search radius & $\begin{array}{l}1660(1 / 2 \text { field } \\
\text { width) }\end{array}$ & $\begin{array}{l}3013 \text { (max interpt } \\
\text { dist) }\end{array}$ & 42.9 (true range) \\
\hline
\end{tabular}

* Geopack is not able to auto-fit any model to these data using the original coordinate system. However, the model fit using the revised coordinate system can be translated to this coordinate system.

\section{Producing Maps in Surfer}

The variogram calculations given in the above tables were performed directly in the software packages. However, neither GeoPack nor GS+ is able to produce presentation-quality maps for displaying the results. (GS+ actually has this capability, but it refuses to display the output when kriging on a 100 by 100 grid). Geopack also crashes when the grid density is greater than 50 by 50 . Therefore, the defaults for each package were entered into Surfer's kriging routine to generate the map files. Results from these kriging runs were cross-validated by comparing results to those reported directly by Geopack and GS+. 
The most peculiar result from these runs related to Geopack. The map produced from the Geopack defaults indicated a scattering of successful kriging results near the core of the input data. Since Geopack substitutes the mean for the kriging estimate when too few kriging neighbors are available at a given location, most of the field defaulted to the mean of the input data.

Originally, we assumed that Geopack's aberrant behavior arose from fitting a weak or invalid variogram to the data. This was logical since we had earlier (iteration \#1, above) managed to produce results in the general range of the Surfer and GS+ results using a truncated variogram distance. However, progressively reducing the variogram distance and fitting new models did little to rectify the problem. This is because the variogram range for every model (from the default $1 / 2$ of the field width down to $\sim 1 / 3$ of the default distance) remained short ( $<4 \%$ of the field width). Since Geopack's default search radius is equivalent to the variogram range, most of the kriging attempts are failures.

The key to improving Geopack's poor performance is to uncouple the search radius from the variogram range. The following plots illustrate the point. Each of the 4 plots on the first page used a different search radius. The plot on the separate page used all of Geopack's defaults, including the default search radius. The pattern is obvious: increasing the search radius leads to fewer kriging failures and produces a kriging map more similar to that of GS+ and Surfer. 



\section{SURFER DEFAULTS}

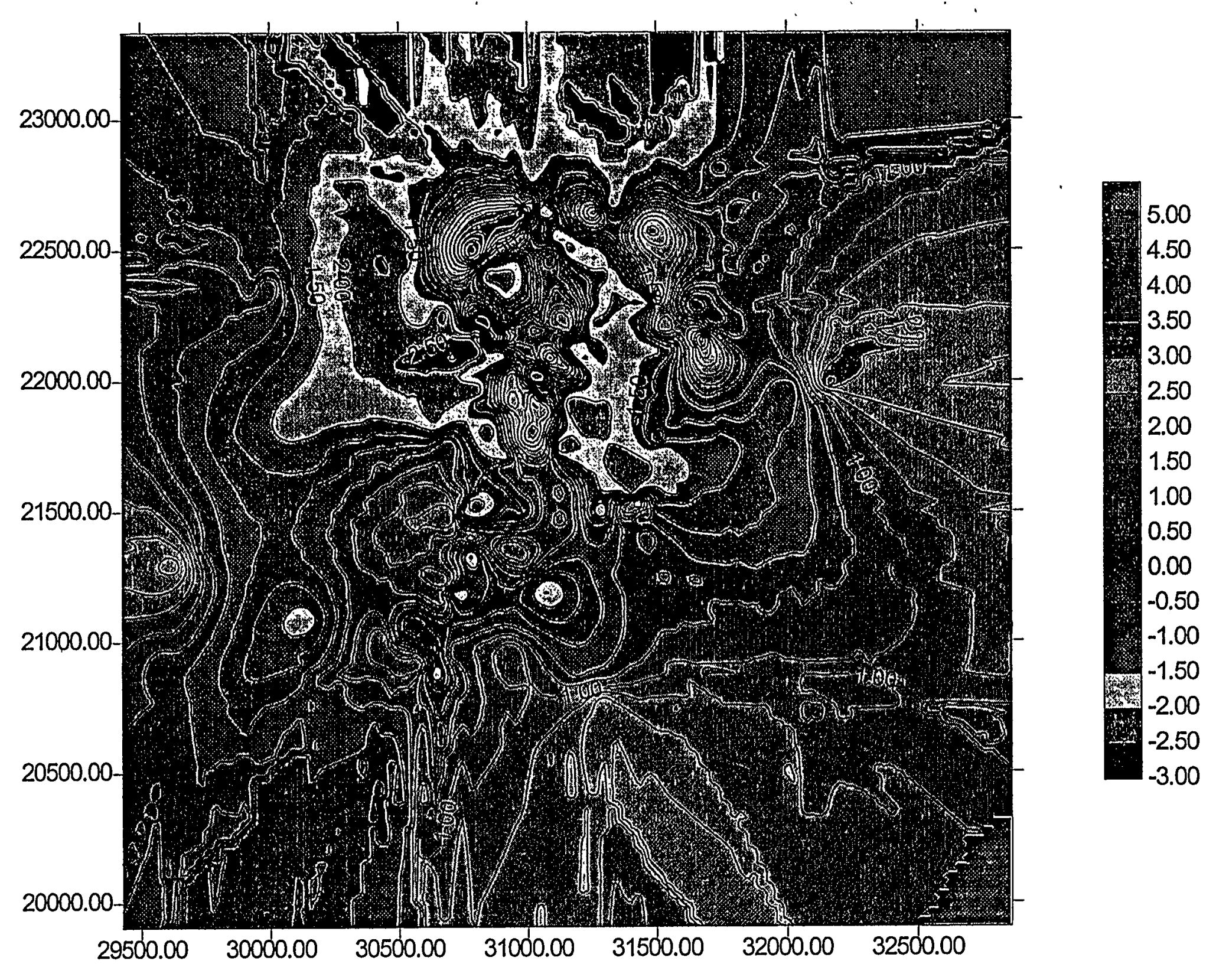




\section{禺

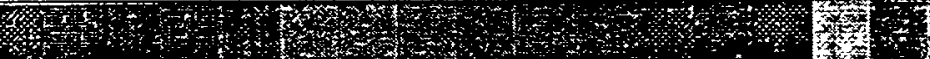

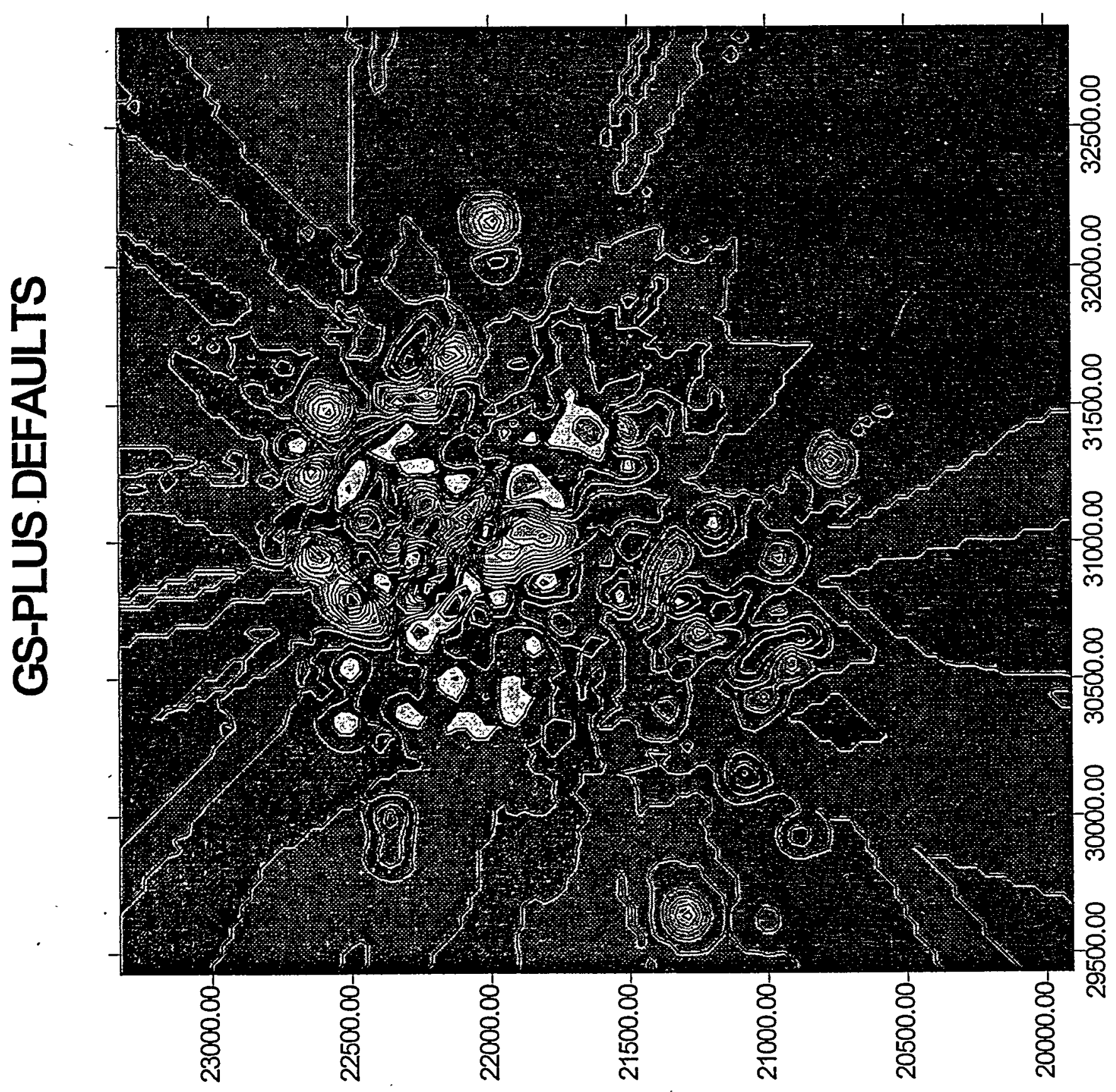


EFFECT OF SEARCH RADIUS ON GEOPACK RESULTS
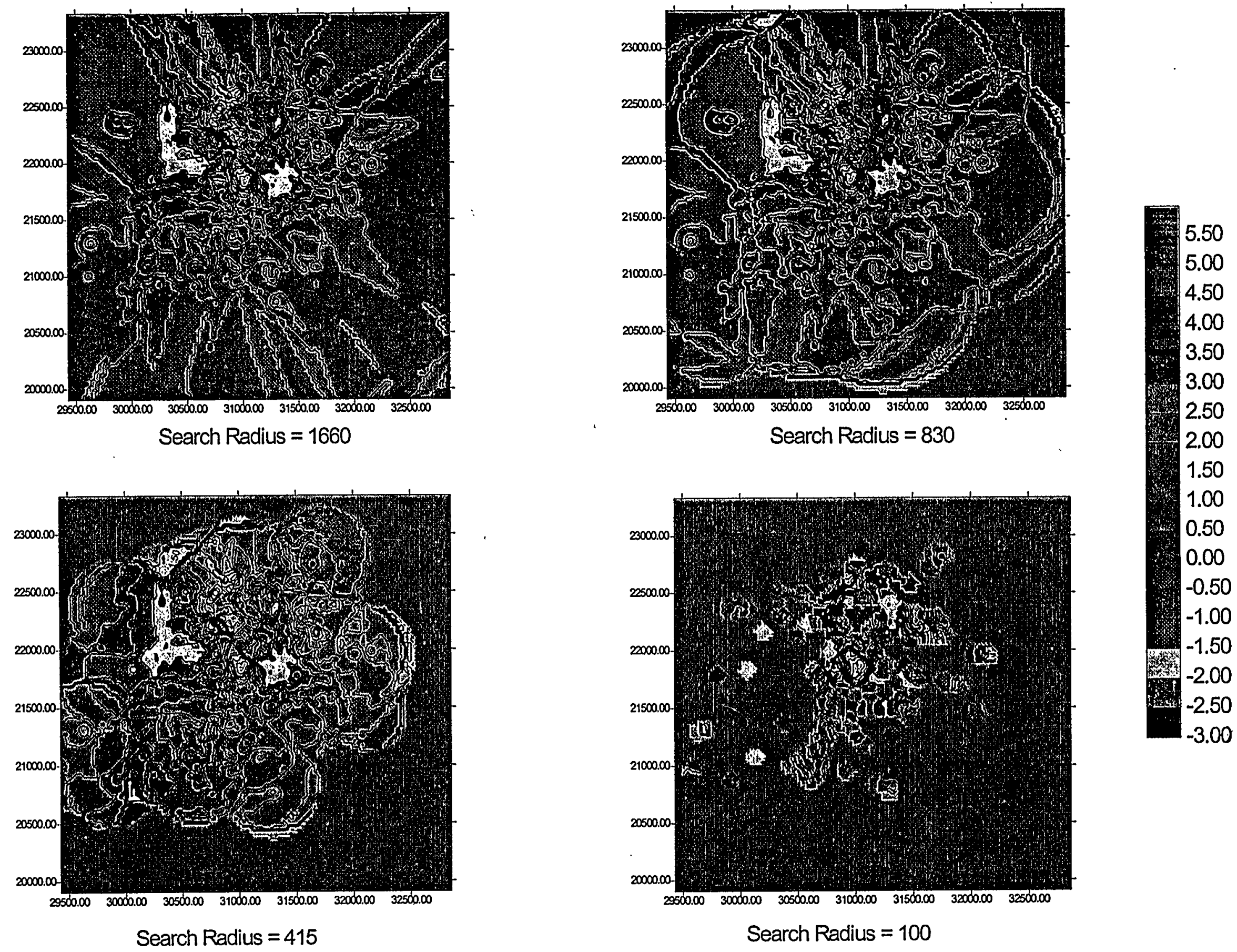


\section{SUMMARY OF SOFTWARE EVALUATION RESULTS}

This memo is intended to summarize the salient points gleaned thus far from the kriging software evaluation project. At this juncture it is worthwhile to take stock of the progress in order to better direct future efforts. This report supplements three reports/memos previously submittted:

- Evaluation of Geostatistical Software Packages

- Overview of Kriging Simulation Results

- How the Software Comparison Maps were Created

\section{A. Simulation Results}

The default settings/parameters we manipulated were:

Detrending

- Linear detrending

- Quadratic detrending

Semivariance Calculation

- Maximum distance used for computing lags

- Number of lags/lag width

Variogram Modeling

- Variogram model (spherical, exponential, gaussian)

\section{Kriging}

- Maximum neighbors

- Minimum neighbors

- Search radius

Following preliminary analysis we added another switch for failure mode. This refers to the manner in which unsuccessful kriging attempts (insufficient neighbors) were handled. The options were: substitute mean of the data for the kriging estimate or skip that point.

Although we anticipated that at least some of the default combinations would render substantially poorer results than others, most of the summary statistics were in accordance with expected values (Results of Kriging Simulations: September 1997). The simulations indicated very little difference in the performance (in terms of the 
summary statistics we selected) among the various defaults used for variogram modeling or kriging. However, in the course of the work we have encountered several anomalies that may merit further investigation. These are described below.

\section{B. Additional Observations from the Simulations or Other Explorations}

For the simulations, we manipulated the default parameters more-or-less independently. Yet the interaction among certain parameters may produce unusual results. In particular, setting the required number of neighbors high (say, 8-10) and the search radius very low leads to a situation where very few locations can be successfully kriged. What to do with the failed kriging attempts is therefore of interest. Some programs (e.g., GeoEas) report NaN and maps produced by the program indicate voids in the kriging estimate grid at those locations. One program (GeoPack) used the mean and standard deviation of the input data as surrogates for the kriging estimate and standard error, respectively. We all agreed that this was flawed logic. It also led to very misleading results in the simulations, since the wide confidence intervals associated with the standard deviations created the erroneous impression of stellar performance!

The search radius/neighbor issue may be largely an artifact of earlier constraints on computer processing power. In the days of $8086 \mathrm{CPU}$ 's and 640K memory limits, there was good reason to restrict computations to those necessary to render sufficient precision without overkill. Now that memory limits are less of a problem, most newer kriging software alleviates the insufficient neighbor problem by automatically setting the search radius very high (up to the maximum interpoint distance) so that enough neighbors are always found. This is the default for S-Plus (the user cannot override this feature) and all of the data points can potentially be used in generating the kriging estimate. Likewise in Surfer, if there are less than 200 data points, all of the data points are used for kriging (i.e., there is essentially no search radius). If there are more than 200 data points, then the search radius is set to $1 / 2$ of the field width and the search type is a quadrant search with 24 total points ( 6 per quadrant). GS+ sets the search radius equal to the maximum interpoint distance and allows up to 64 points for kriging. However, the computations are very slow when kriging on a dense grid (say, 50 by 50 ) if the number of neighbors is set this high. I anticipate that the soon-to-be-released 32bit version of GS+ will rectify the speed issue.

The two DOS programs either behave poorly or crash if the number of neighbors is set too high. The defaults for GeoEas are 16 neighbors drawn from a search radius of $1 / 4$ the maximum interpoint distance. The defaults for GeoPack are 10 neighbors drawn from a search radius equal to the range of the variogram. As we discovered, Geopack's options are the source of some strange behavior if the variogram model (either auto-fit 
or specified by the user) happens to have a very short radius (see report titled How the Software Comparison Maps were Created for an example using the Wag-1 data set).

We noticed a peculiar surface in Surfer's kriging rendition for the Wag-1 data. A region in the southeast corner of the contour plot indicated a very "hot" region. This area was well removed from the data and the intervening area (region between the hot zone and the data) was a lower concentration. Initially, we felt that this anomaly was due to some mathematical overload. However, Golden Software's technical support reported that this anomaly is due to Surfer's manner of handling kriging failures. When too few neighbors are found, it reports a very large number $(1.70141 \mathrm{E}+038)$. For the Wag-1 data, Surfer's default search radius is 1660 -feet ( $1 / 2$ the field width). We extended the kriging grid for these data to an area of 3430-feet by 3430 -feet for which the southeast corner lies approximately 1815 -feet from the nearest data points. This is the source of the kriging failures and the mysterious hot zone on the contour plots.

In summary, we have identified several different ways of handling and reporting kriging failures: $\mathrm{NaN}$ (GeoEas), mean of the input data (GeoPack), arbitrarily large numbers (Surfer), and arbitrarily small numbers (-99.00 in GS-Plus). Setting the estimate to șome impossible number seems like a reasonable option, as long as the user is aware that this has occurred. Displaying some sort of warning message would be advantageous.

We have also encountered a number of potentially confusing aspects of the input and output among programs. These pertain primarily to the manner in which the different programs parameterize the variogram models. Differences in parameterization are probably of greater concern to us than they would be to the average practitioner, since we customarily jump back and forth among the different software packages. However, a casual user could be easily tripped up if, for example, they modeled the variogram in one package and proceeded to enter the variogram parameters in another package which employed a different parameterization. Examples are given below.

The "sill" may refer to two different points on the Z-axis: the "true" sill (the level at which the variogram asymptotes) or the "partial sill" (the difference between the true sill and the nugget). The partial sill, denoted by $C_{s}$, is the value that is parameterized in the most common version of the variogram models. The true sill is then equivalent to the sum of the nugget and the partial sill $\left(\mathrm{C}_{\mathrm{o}}+\mathrm{C}_{\mathrm{s}}\right)$. GS+ "thinks" in terms of the true sill while the other packages generally deal with partial sills. Surfer refers to the partial sill as the "scale."

The other potentially confusing parameter is the range $\left(A_{0}\right)$ for the exponential model. GeoEas, GeoPack, and S-Plus use the traditional geostatistical parameterization 
whereby the "range" equals $1 / 3$ of the distance at which the variogram asymptotes. The asymptotic length is sometimes referred to as the practical range. In GS-Plus and Surfer, the variogram model uses the practical range for all models, including the exponential. Surfer refers to this distance as the "length" while GS-Plus has a block for "Range: $A_{0}$ or $3 A_{0} . "$ 


\title{
ESTIMATION OF SX-FARM VADOSE ZONE Cs-137 INVENTORIES FROM GEOSTATISTICAL ANALYSIS OF DRYWELL AND SOIL CORE DATA
}

\author{
Daniel Goodman \\ Environmental Statistics Group \\ Biology Department \\ Montana State University \\ Bozeman, MT 59717 \\ January 28,2000
}




\title{
ESTIMATION OF SX-FARM VADOSE ZONE Cs-137 INVENTORIES FROM GEOSTATISTICAL ANALYSIS OF DRYWELL AND SOIL CORE DATA *
}

\author{
Daniel Goodman \\ Environmental Statistics Group \\ Biology Department \\ Montana State University \\ Bozeman, MT 59717
}

January 28,2000

\section{INTRODUCTION}

The SX tank farm comprises 15 tanks, some of which have been involved in significant leak events. For many years, the tank farm was monitored by gross gamma logging in 99 vertical drywells, most of which were located near the perimeters of the tanks. More recently, most of these vertical drywells were re-measured by spectral gamma logging. Early in the history of the monitoring, gross gamma logging was carried out in lateral drywells under ten of the tanks (though the lateral logging data available to us were only from eight of these tanks). Additionally, samples were analyzed from soil cores taken near two of the tanks, mostly from positions closer to the tank wall than the perimeter drywells. Figure 1 shows the locations of all wells with data.

* This is a report to MACTEC Inc., under agreement 3751.119.376 with Montana State University. Portions of the work reported here were supported by DOE cooperative agreement DE-FC07-94ID13317 to Montana State University, Daniel Goodman, Principal Investigator. 


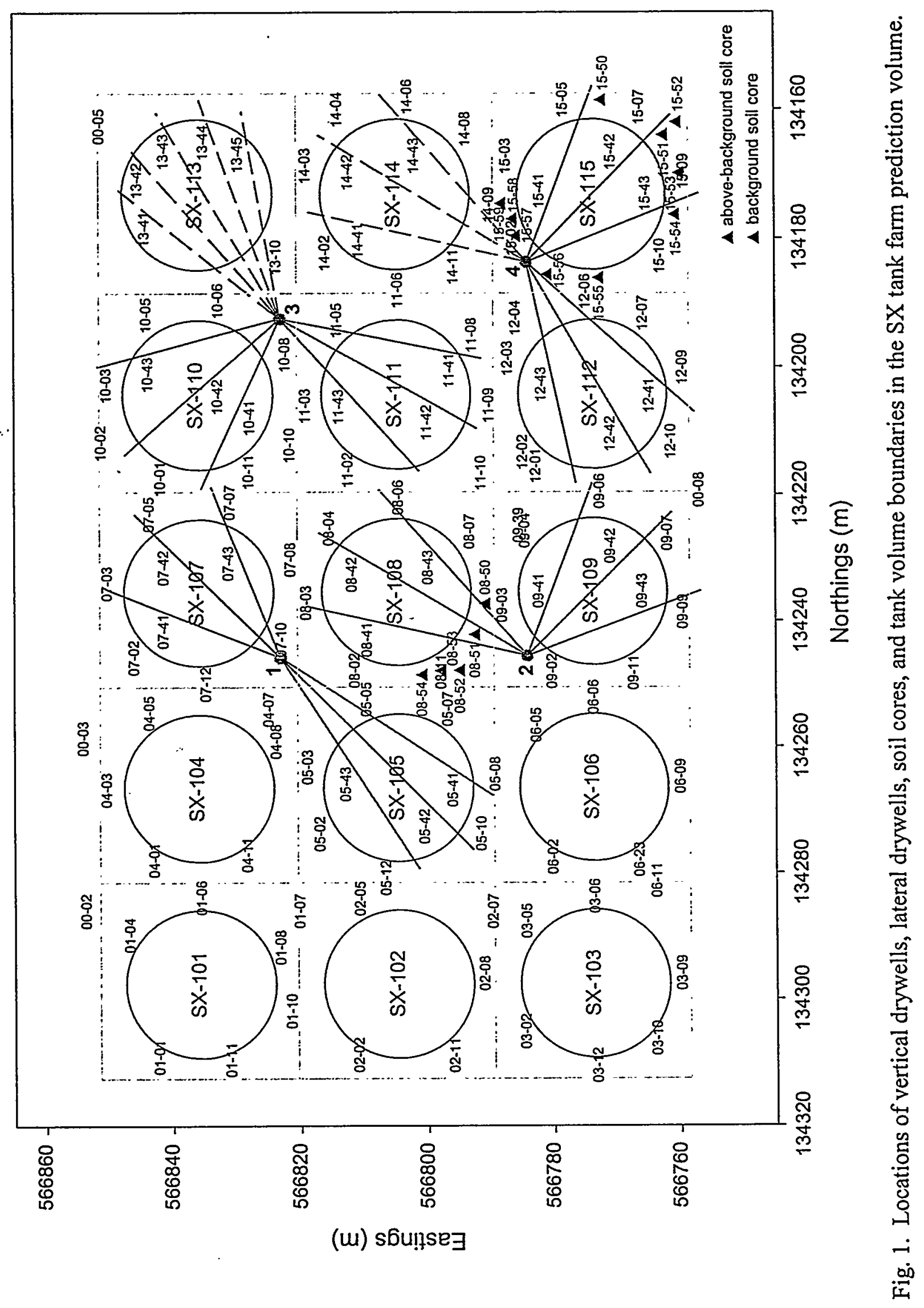


The objective of the present analysis is to estimate Cs-137 inventories in the soil volume under the tanks, from the measurement data from the drywells and soil cores. More specifically, the inventories are to be estimated separately for distinct volumes of soil associated with each tank. Further, the soil volume included in the inventory will be defined by exceedance of specified concentration thresholds.

The geostatistical method used is to be the second-order-stationary Gaussian process, as developed for this type of application in our previous report (Goodman, 1999). This method has the advantage of being comparatively simple and "purely statistical," so it intrudes a minimal number of assumptions into the analysis. In particular, it does not require specification of the physical process responsible for the spatial pattern. We have previously determined that this method exhibits very good stability for predicting point estimates of contamination, for the estimates "track the data." Further, when the "background" contamination level is specified for one of the parameters of the model, this precludes the creation of "phantom hot areas" which can be a disconcerting feature of other geostatistical techniques. For this reason, the inventory estimates will be realistic, but conservative, in the sense of defaulting to predictions of low contamination in locations that are too far from data.

The behavior of this geostatistical method for quantifying the uncertainty (e.g., confidence limits) on inventory estimates in this type of application has not be explored previously, so a secondary objective in the work reported here is to determine the properties, reasonableness, and sensitivity to assumptions of the mathematically computed uncertainty measures.

The data set spans a very large soil volume. The volume within which predictions could be made is 175 (N-S) $\times 120$ (E-W) $\times 50$ meters (depth), for a total of slightly over one million cubic meters. Data were available for about 19,610 distinct locations in this volume. For reasons that will be explained later, the data were aggregated into averages within each cubic meter that contained data, resulting in 3,962 cells that contained data which could be used for interpolation or prediction. This may seem like a lot of data, yet at the resolution of 1 cubic meter, we see that only $0.4 \%$ of the cells in the total volume actually had data in them, and the remaining $99.6 \%$ of the cells needed to have their value estimated by geostatistical interpolation.

Furthermore, the cells that contain data are not randomly or uniformly dispersed through the volume. The data are confined to horizontal or vertical strings of cells, with the consequence that a large fraction of the cells in the total volume can be more than 5 meters from the nearest data location. 
The combination of large prediction volume, large numbers of data locations, and large fraction of the prediction volume being distant from data locations, strains the predictive ability of geostatistical theory, and strains the computational machinery for implementing the geostatistics. Accordingly, there was a premium on using credible geostatistical methods that were, to the extent possible, simple, transparent, and robust, both with respect to the calculation of point estimates and with respect to the quantification of uncertainty. Our general approach for geostatistical analysis, but not including inventory estimation, is documented in the September 1999 report to MACTEC (Goodman, 1999).

The data available spanned a long history and involved measurements with a substantial variety of instruments for which specific cross-calibration information was not available to us. Some of these instruments had very different sensitivities, so that some were incapable of much resolution at low concentrations, while others would saturate at high concentrations. Of the values used to compute cell averages for the $\mathrm{m}^{3}$ cells that had data, 7,721 were obtained as spectral gamma measurements reported as $\mathrm{pCi} / \mathrm{g}$ Cs-137, 11,591 were obtained as spectral gamma total gamma reported as counts, 177 were obtained as measured Cs- 137 from soil samples reported in $\mathrm{pCi} / \mathrm{g}$, and 3,583,were either gross gamma reported in counts with various historical instruments or values that we set to "background" because a soil sample reported "not above trace" but was not quantified further. Thus, assembly of a comprehensive merged data set for the geostatistical analysis required a complicated sequence of decisions about data selection and data conversion.

In the present report package we:

1. document the assembly of the data set used for inventory estimation (Appendix 1),

2. deliver a copy of the data set (electronic file, on disk),

3. document the specific approach used for inventory estimation (Appendix 2), and

4. present the results of the inventory calculations (Appendix 3).

The method used, as documented in Appendix 2, involved minimal assumptions, but not all these assumptions were necessarily consistent with all the features of the data. The assumptions allowed the estimates to track the actual data very well, so the inventory estimates were robust and reasonable. If anything, the inventory estimates are perhaps underestimates, because of the absence of data 
directly under the tanks at depths deeper than the laterals (some of the vertical drywells show that substantial contamination can be found at much greater depth in some locations). The uncertainty quantification, using these methods and assumptions, was not as robust, because of sensitivity to some of the assumptions that were not entirely consistent with the data.

Refinement of the inventory estimate to better correct for the effect of the "data holes" under the laterals, and development of more stable quantification of uncertainty, could both be accomplished by a more complicated modeling approach, that takes into account more of the physical properties of the plume geometry, as described in Appendix 2. This would entail more assumptions than the model used in the present report, but those assumptions could be tailored to be more consistent with the actual process and the data.

The logical next step to pursue in the vadose zone geostatistical analysis is to extend the methodology specifically to cope with the inventory estimation in the other tank farms that do not have laterals. The absence of lateral data creates larger "data holes" under the tanks, which might create a significant bias in inventory estimates if it were not corrected. The SX-Farm data set presents an opportunity to develop a calibration of effects of presence or absence of lateral data, and this calibration could then be extrapolated to other tank farms where there are no lateral data.

\section{REFERENCES}

Goodman, D. 1999. Documentation of Geostatistical Approach for the Analysis of SX-Farm Vadose Zone Gamma Data. September 27, 1999, report to MACTEC Inc., under agreement 3751.119.376 with Montana State University. 


\section{Table of Contents}

I. Estimation Of SX-Farm Vadose Zone Cs-137 Inventories From Geostatistical Analysis Of Drywell And Soil Core Data

II. Appendix 1-Assembly Of The Composite Data Sets

III. Appendix 2-Geostatistical Approach Used In Estimation Of SX-Farm Vadose Zone Cs-137 Inventories

IV. Appendix 3-Results Of The SX Inventory Calculation 


\section{List of Figures - Appendix 1}

Page

1. Locations of vertical drywells, lateral drywells, soil cores, and tank volume

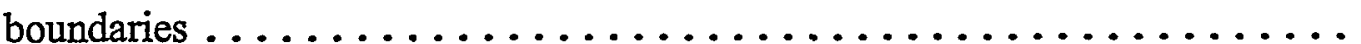

2. Histogram of 1995 gamma-logging values less than $50 \mathrm{cps} \ldots \ldots \ldots \ldots$

3. Historical gamma logging values vs. spectral gamma total counts . . . . . . . 1-5

4. Drywell 09-39 gamma logging with spectral gamma and LVMS detectors . . . . 1-6

5. Historical gamma logging values vs. Cs-137 concentrations . . . . . . . . . . 1-10

6. Histograms of all values in the composite SX data set, broken down by probe



7. Histogram of all values less than $100 \mathrm{cps} \ldots \ldots \ldots \ldots \ldots \ldots \ldots \ldots \ldots$

8. Histograms of vertical drywell gamma counts $\ldots \ldots \ldots \ldots \ldots \ldots \ldots$

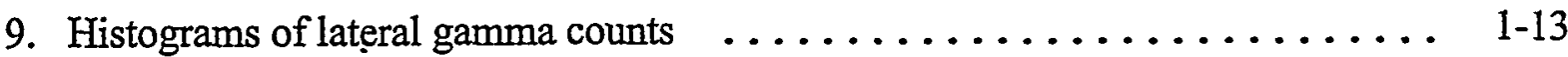

10. Distributions of maximum counts per well $\ldots \ldots \ldots \ldots \ldots \ldots \ldots \ldots$. . . . . . . . .

11. Histogram of soil core values and distribution of maximum values $\ldots \ldots \ldots$ 1-15

12. Histograms of vertical, lateral, and all gamma logging values and binning $\ldots \ldots$ 1-16

13. Spectral gamma total counts . . . . . . . . . . . . . . . . . . 1-17

14. Spectral gamma $\mathrm{Cs}-137 \mathrm{pCi}^{-1}$ vs. spectral gamma total counts $\ldots \ldots \ldots$. . . 1-18

15. Spectral gamma Cs-137 $\mathrm{pCi} \mathrm{g}^{-1}$ vs. spectral gamma total counts in type 4 units and SX-108 soil core $\mathrm{Cs}-137 \mathrm{pCig}^{-1}$ vs. kriging-predicted type $4 \mathrm{cps}$. . . . . . . 1-19

16. $\mathrm{SX}-115$ soil core $\mathrm{Cs}-137 \mathrm{pCi}^{-1}$ vs. kriging-predicted type $4 \mathrm{cps} \ldots \ldots$. . . . 1-20

17. Histograms of all values in the composite Cs-137 data set, broken down by

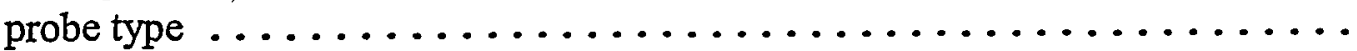

18. Histograms of vertical drywell values in the composite Cs-137 data set, broken down by probe type .........................

19. Histograms of lateral drywell values in the composite Cs-137 data set, broken down by probe type . . . . . . . . . . . . . . . . . . . . . . 1-23

20. Histograms of the binned composite Cs-137 data set, bin sample variance and

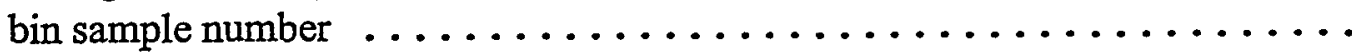

21. Drywell 09-39 $\mathrm{Cs}-137 \mathrm{pCi}^{-1}$ from gamma logging and soil cores $\ldots . . . \ldots \quad$ 1-25

\section{List of Tables - Appendix 1}

1. Conversion of gamma counts from four different detectors . . . . . . . . . 1-6

2. Patches applied to vertical drywells in the SX spectral gamma data set . . . . . 1-7

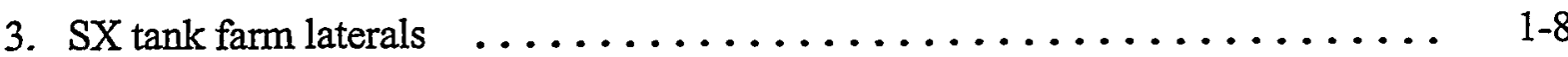

4. Soil core labels in Fig. 1 and Raymond and Shdo (1966) . . . . . . . . . . . 1-9

5. Conversion of gross gamma counts . . . . . . . . . . . . . . . . . 1-21 
List of Figures - Appendix $2 \quad$ Page

1. Cell sample variance vs. cell mean $\ldots \ldots \ldots \ldots \ldots \ldots \ldots \ldots \ldots \ldots .2-4$

2. Histogram of 1994 gamma-logging values less than $50 \mathrm{cps} \ldots \ldots \ldots \ldots . \ldots 2-6$

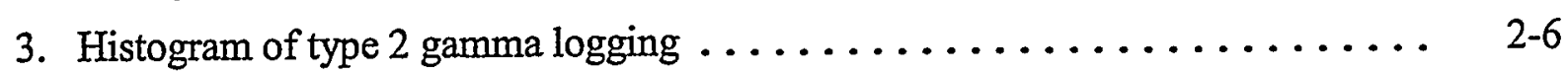

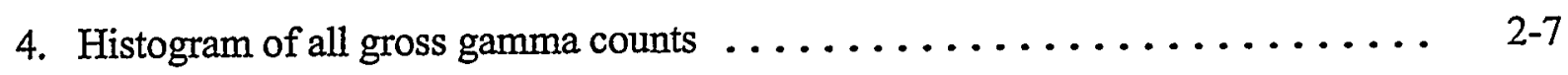

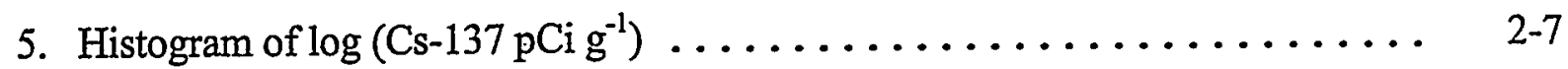

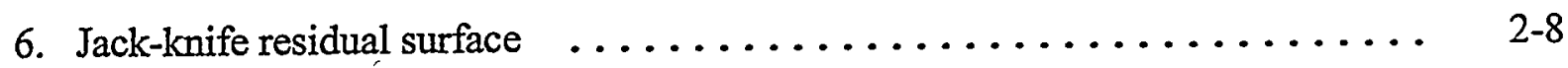

7. Spectral gamma Cs-137 $\mathrm{pCi} \mathrm{g}^{-1}$ vs. spectral gamma total counts $\ldots \ldots \ldots .2-21$

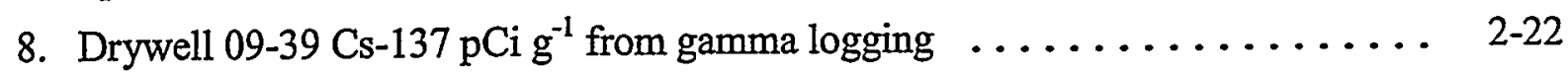

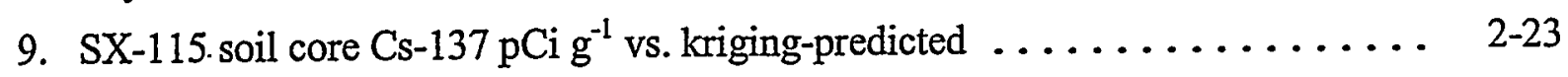

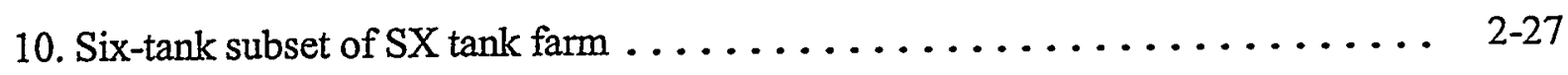




\section{List of Figures - Appendix 3}

1. Sx Kriging point predictions exceeding $5 \times 10^{1} \mathrm{pCi} / \mathrm{g} \ldots \ldots \ldots \ldots \ldots \ldots$

2. SX Kriging point predictions exceeding $5 \times 10^{2} \mathrm{pCi} / \mathrm{g}$

3. SX Kriging point predictions exceeding $5 \times 10^{3} \mathrm{pCi} / \mathrm{g}$.

4. SX Kriging point predictions exceeding $5 \times 10^{4} \mathrm{pCi} / \mathrm{g}$

5. SX Kriging point predictions exceeding $5 \times 10^{5} \mathrm{pCi} / \mathrm{g}$

6. SX Kriging point predictions exceeding $5 \times 10^{6} \mathrm{pCi} / \mathrm{g}$

7. SX Kriging point predictions exceeding $5 \times 10^{7} \mathrm{pCi} / \mathrm{g}$.

\section{List of Tables - Appendix 3}

1. SX tank farm inventory of soil volumes

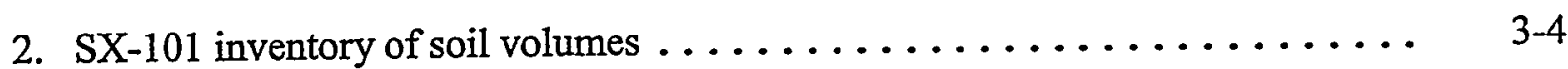

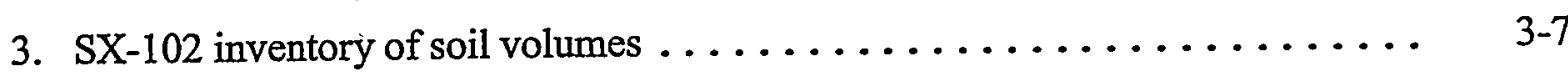

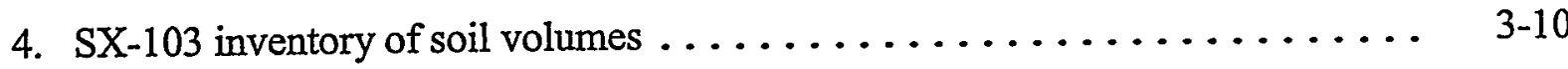

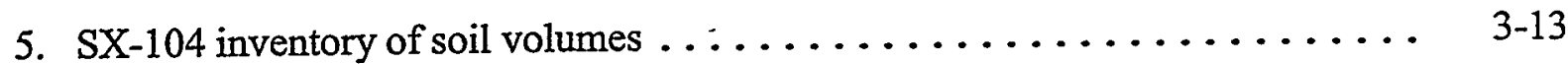

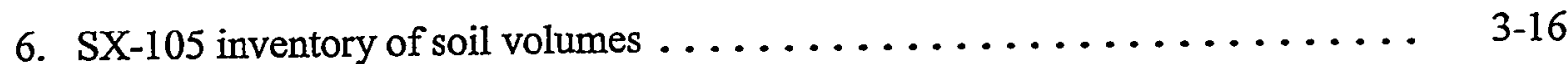

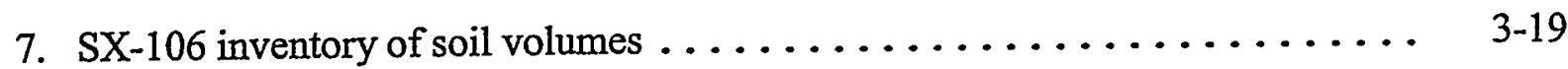

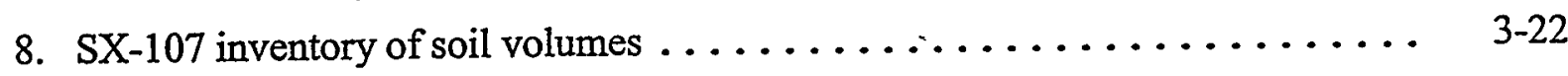

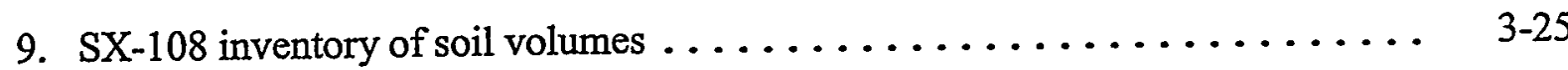

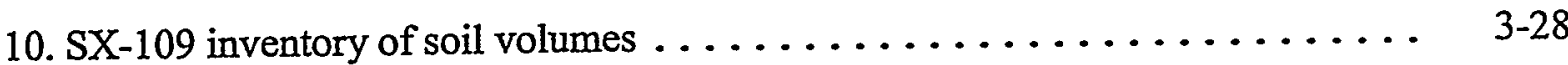

11. SX-110 inventory of soil volumes $\ldots \ldots \ldots \ldots \ldots \ldots \ldots \ldots \ldots \ldots .3-31$

12. SX-111 inventory of soil volumes $\ldots \ldots \ldots \ldots \ldots \ldots \ldots \ldots \ldots \ldots . \quad 3-34$

13. $\mathrm{SX}-112$ inventory of soil volumes $\ldots \ldots \ldots \ldots \ldots \ldots \ldots \ldots \ldots \ldots .3-37$

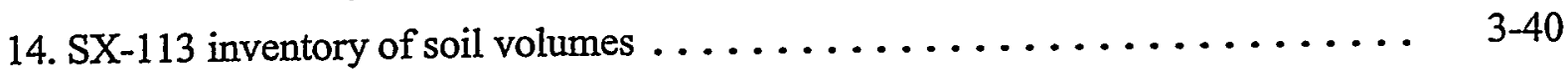

15. $\mathrm{SX}-114$ inventory of soil volumes $\ldots \ldots \ldots \ldots \ldots \ldots \ldots \ldots \ldots \ldots . \ldots \ldots \ldots . \ldots \ldots$

16. SX-115 inventory of soil volumes $\ldots \ldots \ldots \ldots \ldots \ldots \ldots \ldots \ldots \ldots .3-46$ 


\section{APPENDIX 1. ASSEMBLY OF THE COMPOSITE DATA SETS}

Two data sets were compiled over the course of the SX tank farm inventory project. Both data sets are composites of spectral gamma and historical gamma logging values from 99 vertical drywells in combination with historical gamma logging at 24 lateral wells and a small number of core samples. The first data set, the "composite gamma counts data set", is composed of gamma activities in counts per second (cps) while the second data set, the "composite Cs-137 data set", contains Cs-137 concentrations in $\mathrm{pCi}^{-1}$ of soil. The composite gamma counts data set was initially used in exploratory parameter estimation and later used in a slightly modified form to derive a conversion from gross gamma logging in cps to Cs-137 concentrations. Importantly, the composite Cs-137 data set was the data set actually used to geostatistically calculate the SX tank farm inventory.

\section{The soil volume of interest}

All values in the two composite data sets were collected within a $175 \mathrm{~m}$ (north-south) by $120 \mathrm{~m}$ (east-west) by $50 \mathrm{~m}$ soil volume encompassing the fifteen SX storage tanks (Fig. 1). The southern boundary of the volume is located at northing $134145 \mathrm{~m}$ with the western boundary at easting $566745 \mathrm{~m}$. The top of the volume was set at the elevation of the top of the highest drywell in the SX-farm (well 01-07 at $203.61 \mathrm{~m}$ ). The soil volume was further subdivided into 15 equally sized subvolumes ( $31 \mathrm{~m}$ by $31 \mathrm{~m}$ by $50.5 \mathrm{~m}$ deep) approximately centered around each tank in the SX farm (Fig. 1). Cs-137 inventories assigned to each of the fifteen tanks were compiled within these subvolumes.

\section{Drywell and lateral locations}

In reconciling discrepancies between drywell $\mathrm{x}-\mathrm{y}$ coordinates provided by Mactec-Meier (Steve Anderson, pers. comm., 10/5/98) and the geologic strata data set obtained from Dave Meyers (pers. comm., 11/24/98), we used drywell coordinates contained in the Bechtel Hanford, Inc. GIS (Dave Meyers, pers. comm., 2/6/99). However, the Bechtel database did not contain a complete record of drywell elevations at the SX farm. Since the complete set of drywell elevations provided by Mactec-Meier closely resembled Bechtel elevations where comparisons could be made, the Mactec-Meier elevations were combined with the Bechtel $x-y$ coordinates. Two recently bored drywells, 09-39 and 12-01, were not included in the Bechtel database, thus requiring use of Mactec-Meier $\mathrm{x}-\mathrm{y}$ coordinates. Lateral locations were assigned using caisson coordinates in tandem with well lengths, orientations, and depths summarized in Hanford drawing number H-2-31881, "241-SX tank farm leak detection system plan." Assignment of $\mathrm{x}$ $y-z$ coordinates to lateral data was complicated by the manner in which that data was reported, i.e. in distances from recording points to the surface via individual laterals and the caisson from which they originated. Using Hanford drawing number H-2-31882, "241 A \& 241 SX tank farms leak detection system: sections \& details", we calculated a total distance of $62.11 \mathrm{ft}$. from the surface to where laterals exit their respective caissons. Any gamma counts recorded at distances smaller than this value were treated as internal to a caisson and ignored. Vertical and lateral drywell locations are plotted in Fig. 1. Vertical drywell labels are identical to designations commonly used at Hanford minus the leading " 41 " typically designating the SX 


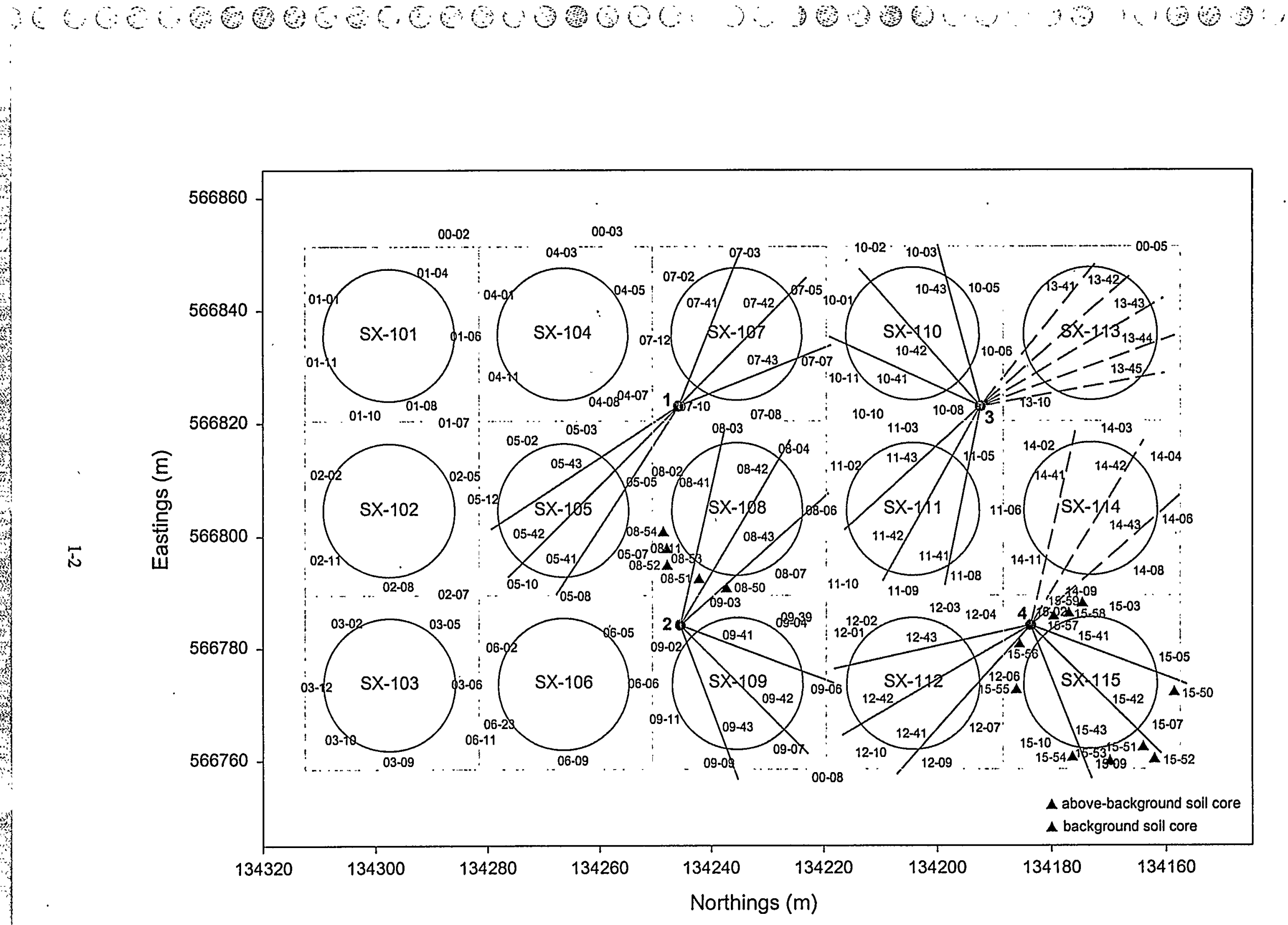

Fig. 1. Locations of vertical drywells, lateral drywells, soil cores, and tank volume boundaries in the SX tank farm prediction volume. 
tank farm. At Hanford, lateral drywells are commonly identified by their tank number followed by a lateral number, 1-3 (WHC, 1992a; WHC, 1992b). In our naming scheme, laterals are identified by their tank number followed by an integer, 41 through 43 , corresponding to laterals 1-3. One exception to this rule is SX-113 with five laterals labeled 13-41 through 13-45. Where gamma logging data was available, laterals are plotted with solid lines in Fig. 1. Data from eight laterals associated with SX-113 and SX-114 was unavailable and thus these laterals are drawn with dashed lines.

\section{The composite gamma counts data set}

\section{Gamma logging probes and their cross-calibration}

Gamma logging values included in the composite gamma counts data set were acquired with six different instruments. Typically, gamma logging at vertical drywells in the SX tank farm was conducted with the type 4 (NaI-scintillation) probe. At higher levels of radiation, the type 14 (shielded NaI-scintillation), type 1 (green Geiger-Muller), or type 2 (red Geiger-Muller) probes were used (Isaccson, 1982). Laterals were logged with probe types 1 and 2 (WHC, 1992a; WHC 1992b). Spectral gamma-ray logging system (SGLS) counts were recorded with the high-purity germanium detector (DOE, 1996). A new instrument, the Leak Verification and Monitoring System (LVMS) NaI detector was used at one vertical drywell, 09-39, in January of 1997 (DOE, 1997). All historical gamma logging values (probe types 1, 2, 4, and 14) from vertical drywells were compiled in a Microsoft Access database received from Mactec-Meier (James Kelly, pers. comm., 7/17/98). Spectral gamma counts from all vertical drywells and LVMS data from well 09-39 were received from Mactec-ERS via Mactec-Meier (James Kelly, pers. comm., 9/19/98). Lateral gamma counts (probe types 1 and 2) were compiled by Russ Randall (pers. comm., 5/14/99).

The composite gamma data set was assembled largely from counts logged in 1995 since that was the year spectral gamma logging was conducted at the SX farm (DOE, 1996). All other values in the data set recorded prior to 1995 were half-life corrected to nominal 1995 values using the half-life of Cs-137, 30.17 years. Thus geostatistical analysis of the composite data set can be interpreted as the nominal "best estimate" of the spatial distribution of radioactive contamination at the SX tank farm, circa 1995. Importantly, any improvement in our nominal 1995 "best estimate" resulting from inclusion of data collected prior to 1995 rests on the assumption that radioactive decay is the most important or only process responsible for changes in Cs-137 activity at specific locations over the time interval of half-life correction. This is likely a reasonable assumption given that all values in the composite data set, with the exception of soil core data collected in 1966 (Raymond and Shdo,1966), were collected after the documented leak events at the SX-farm (DOE, 1996) and given the low mobility of Cs-137 (Jones et al., 1998).

Zero values recorded by the six gamma detectors were problematic in that geostatistical calculations were conducted after log-transformation of the data. For clarity, whenever we mention log-transformation or a log value within this report, we are referring to a natural 
logarithm. To allow reasonable log-transformation, we translated zeroes to a nominal "background" value of $25 \mathrm{cps}$ in type 4 units. This "background" value was based on a previous analysis of a six-tank neighborhood surrounding SX-108 and SX-109. A histogram of 1994 gamma counts from all drywells logged with the type 4 probe within this neighborhood indicates that $25 \mathrm{cps}$ is the most likely level of nominal "background" gamma radiation (Fig. 2). Note that translation of zero values to "background" level counts had no effect on the final SX inventories, given that inventories were calculated for Cs-137 activities well above "background."

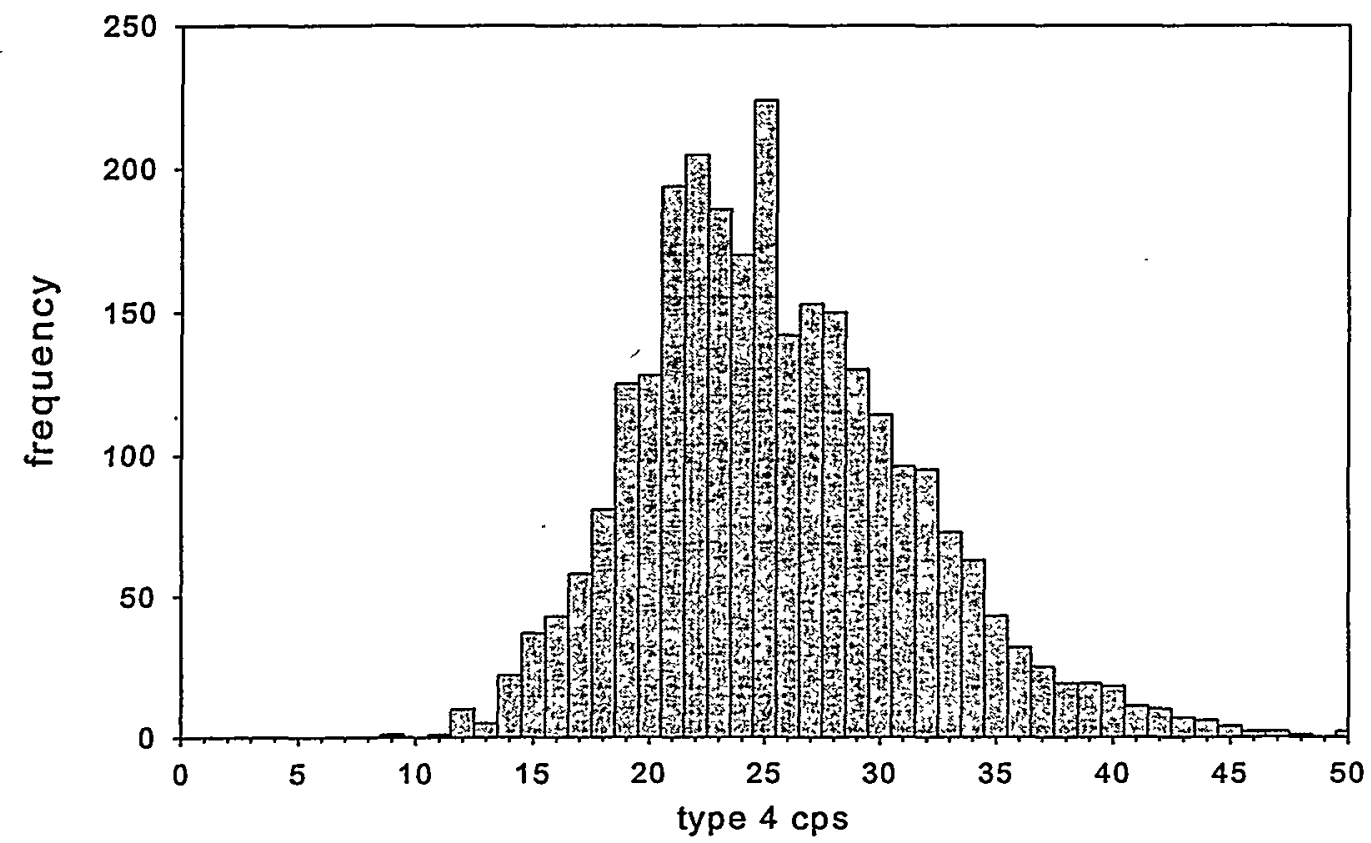

Fig. 2. Histogram of 1995 gamma-logging values less than $50 \mathrm{cps}$ recorded with the type 4 detector in the SX-108, SX-109 six-tank neighborhood.

In assembling the composite gamma data set, all values reported with various instruments were converted to probe type 4 units. Type 1 and type 2 counts were converted using the 1980 "Dry well survey probe calibration" received from Russ Randall (pers. comm., 7/31/67). Per Russ Randall's recommendation (pers. comm., 9/16/98), all type 14 gross gamma values were multiplied by seven. Spectral gamma total counts were converted to gross gamma counts in type 4 units using a calibration relationship fit to a scatter plot of historical gamma logging counts versus spectral gamma total counts (Fig. 3). In creating this plot, spectral gamma data from all vertical drywells in the SX farm was paired with historical gamma values from locations (well/depth) where both types of data were collected. Our search of the historical gamma logging record was restricted to values collected after January 1,1990 . Where location matches could be made, the most recent gross gamma counts were paired with the corresponding spectral gamma value. A maximum of ten historical gamma values were paired with each spectral gamma value with the intention that up to ten repeated measures would be matched with individual spectral gamma counts. Type 1,2, and 14 counts were converted to type 4 units and 





all historical values were half-life corrected to 1995 values. Because the type 2 detector recorded a relatively large number of zero values, type 2 counts were averaged before being paired with spectral gamma values. If the type 2 probe recorded only zeroes, the average was set at the nominal "background" of 25 cps.

Formulas used in cross-calibration of gamma detectors are summarized in Table 1. Note that the LVMS detector has not been cross-calibrated with other instruments used at the SX tank farm. However, spectral gamma counts (in type 4 units) at this well correspond closely to LVMS data (Fig. 4) and so the LVMS data were used without conversion.

Table 1. Conversion of gamma counts from four different detectors to probe type 4 units.

\begin{tabular}{|l|l|}
\hline Gamma logging source & Conversion to probe type 4 cps \\
\hline spectral gamma & $\exp \{-1.3164+0.8784 * \log ($ spectral gamma cps) $\}$ \\
\hline probe type 1 & $\exp \{2.7518+0.9720 * \log ($ type $1 \mathrm{cps})\}$ \\
\hline probe type 2 & $\exp \{8.2745+0.9720 * \log ($ type $2 \mathrm{cps})\}$ \\
\hline probe type 14 & $7 *($ type $14 \mathrm{cps})$ \\
\hline
\end{tabular}

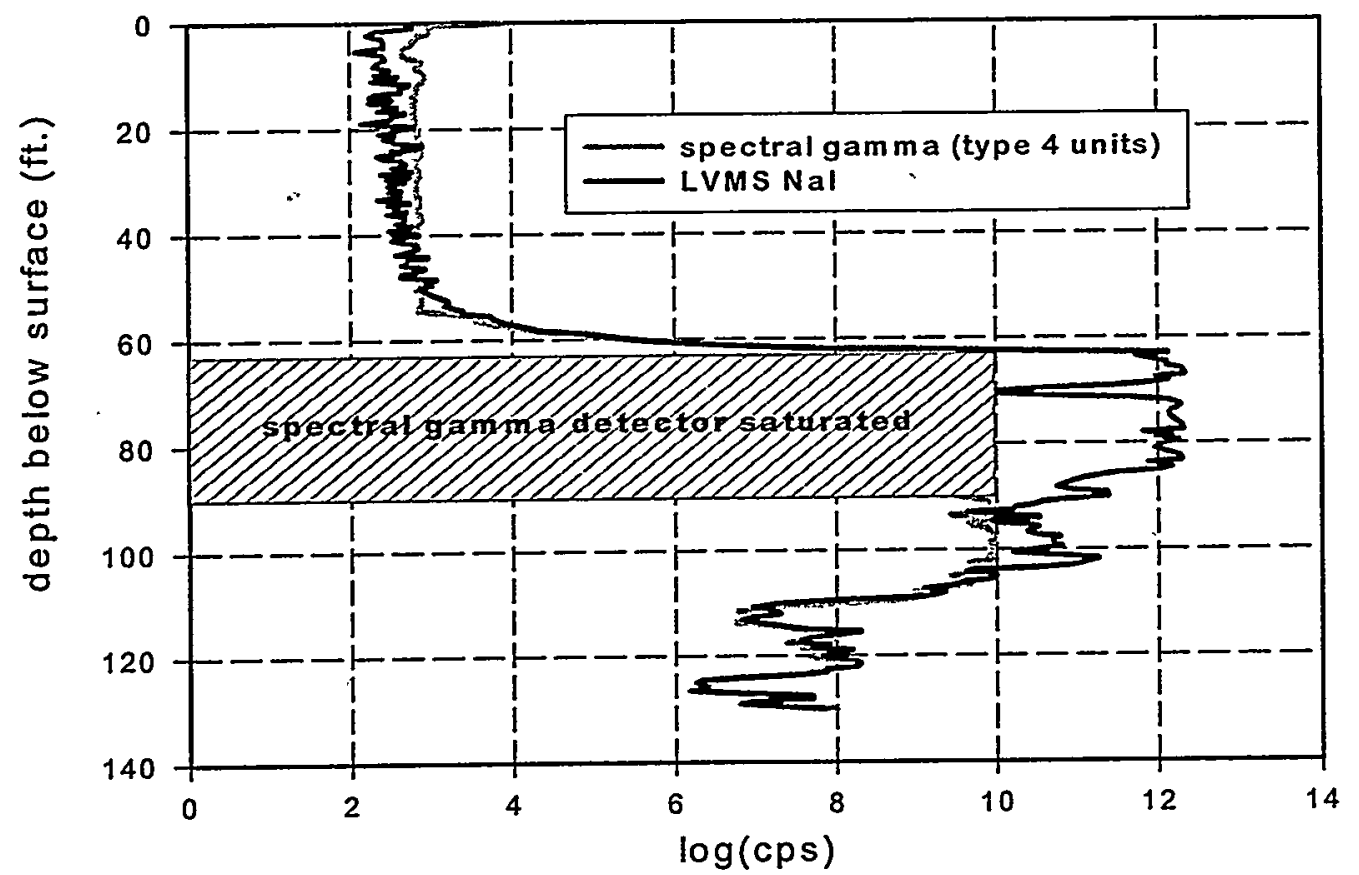

Fig. 4. Drywell 09-39 gamma logging with spectral gamma and LVMS detectors. 


\section{Vertical and lateral gamma logging values}

Vertical drywells

The vast majority of values ( $82 \%$ ) in the composite gamma data set are spectral gamma total counts logged by Mactec-ERS at vertical drywells from August to November of 1995 (DOE, 1996). Drywell 12-01, a companion to well 12-02, was drilled and sampled with the spectral gamma detector in July, 1996 (Brodeur, 1996). Drywell 09-39, a companion to well 0904, was drilled in December, 1996 and subsequently logged with both the spectral gamma and LVMS detectors (DOE, 1997). All zero values (six in total) were transformed to the nominal "background" of 25 type $4 \mathrm{cps}$. Where the spectral gamma data was incomplete due to detector saturation, high dead times, or other reasons, historical gamma logging data was patched into gaps. Details of these patches are summarized in Table 2. At each data gap, the most recent gross gamma logging values were substituted. Where patches composed of type 2 counts contained zero values, the most recent data from a type 1 or type 4 detector was inserted. All patch values were normalized to type 4 units and then half-life corrected to 1995 values.

Table 2. Patches applied to vertical drywells in the SX spectral gamma data set.

\begin{tabular}{|l|l|l|l|l|}
\hline Drywell ID \# & $\begin{array}{l}\text { Reason for data } \\
\text { gap }\end{array}$ & Patch depth(s) & $\begin{array}{l}\text { Patch } \\
\text { date }\end{array}$ & $\begin{array}{l}\text { Probe } \\
\text { type }\end{array}$ \\
\hline $41-00-03$ & well not logged & entire well & $11 / 3 / 93$ & 4 \\
\hline $41-00-05$ & well not logged & entire well & $6 / 11 / 93$ & 4 \\
\hline $41-00-08$ & none provided & $3-61,86-123 \mathrm{ft}$. & $10 / 19 / 93$ & 4 \\
\hline $41-05-08$ & none provided & $6-9 \mathrm{ft}$. & $10 / 25 / 93$ & 4 \\
\hline $41-07-05$ & detector saturated & $55-58 \mathrm{ft}$. & $6 / 10 / 94$ & 2 \\
\hline $41-07-07$ & detector saturated & $56-65 \mathrm{ft}$. & $6 / 10 / 94$ & 2 \\
\hline $41-07-10$ & well not logged & entire well & $6 / 10 / 94$ & 4 \\
\hline $41-08-07$ & detector saturated & $63-72 \mathrm{ft}$. & $6 / 10 / 94$ & 2 \\
\hline $41-08-11$ & detector saturated & $49-61 \mathrm{ft}$. & $6 / 10 / 94$ & 2 \\
& & $62 \mathrm{ft.}$ & $12 / 18 / 91$ & 4 \\
\hline $41-09-07$ & high dead time & $64-72 \mathrm{ft}$. & $6 / 10 / 94$ & 1 \\
\hline $41-09-09$ & high dead time & $72-73 \mathrm{ft}$. & $6 / 9 / 94$ & 14 \\
\hline $41-11-10$ & high dead time & $66-70 \mathrm{ft}$. & $6 / 10 / 94$ & 1 \\
\hline $41-12-02$ & high dead time & $68-69,114,116-117,119 \mathrm{ft}$. & $5 / 27 / 94$ & 4 \\
& & $70-106,115,118,120-122 \mathrm{ft}$. & $6 / 10 / 94$ & 2 \\
\hline $41-12-03$ & high dead time & $65 \mathrm{ft}$. & $6 / 10 / 94$ & 14 \\
\hline
\end{tabular}




\section{Lateral drywells}

SX lateral counts were recorded both electronically and on strip charts. At the 12 laterals where electronically recorded data was available, the most recent set of gamma counts was included in the data set. Otherwise, we used strip chart values from 1975 and 1976 digitized by Russ Randall. All pre-1978 type 2 counts were multiplied by 0.36 to compensate for instrument design changes in sensitivity (Randall, 1999). This re-scaling was limited to lateral data as the vertical drywell data did not contain any pre-1978 type 2 counts. All zero values were replaced with the nominal "background" of 25 type $4 \mathrm{cps}$ and all pre-1995 values were half-life corrected. The sampling date, detector type, recording method, and number of data points from each lateral are summarized in Table 3.

Table 3. SX tank farm laterals.

\begin{tabular}{|l|l|l|l|l|l|l|}
\hline Tank & $\begin{array}{l}\text { Lateral } \\
\text { ID }\end{array}$ & $\begin{array}{l}\text { Sample } \\
\text { date }\end{array}$ & $\begin{array}{l}\text { Detector } \\
\text { type }\end{array}$ & $\begin{array}{l}\text { Recording } \\
\text { method }^{1}\end{array}$ & $\begin{array}{l}\text { \# data } \\
\text { points }\end{array}$ & $\begin{array}{l}\text { \# points set to } \\
\text { "background" }\end{array}$ \\
\hline SX-105 & $05-41$ & $9 / 19 / 89$ & 1 & $\mathrm{e}$ & 122 & 14 \\
\hline & $05-42$ & $9 / 19 / 89$ & 1 & $\mathrm{e}$ & 131 & 13 \\
\hline & $05-43$ & $9 / 19 / 89$ & 1 & $\mathrm{e}$ & 122 & 19 \\
\hline SX-107 & $07-41$ & $6 / 11 / 86$ & 2 & $\mathrm{e}$ & 89 & 63 \\
\hline & $07-42$ & $6 / 11 / 86$ & 2 & $\mathrm{e}$ & 98 & 60 \\
\hline & $07-43$ & $6 / 11 / 86$ & 2 & $\mathrm{e}$ & 89 & 48 \\
\hline SX-108 & $08-41$ & 1976 & 2 & $\mathrm{~s}$ & 180 & 5 \\
\hline & $08-42$ & 1976 & 2 & $\mathrm{~s}$ & 228 & 0 \\
\hline & $08-43$ & 1976 & 2 & $\mathrm{~s}$ & 149 & 1 \\
\hline SX-109 & $09-41$ & $2 / 2 / 76$ & 2 & $\mathrm{~s}$ & 135 & 7 \\
\hline & $09-42$ & $2 / 2 / 76$ & 2 & $\mathrm{~s}$ & 114 & 3 \\
\hline & $09-43$ & $2 / 2 / 76$ & 2 & $\mathrm{~s}$ & 79 & 2 \\
\hline SX-110 & $10-41$ & $8 / 7 / 89$ & 1 & $\mathrm{e}$ & 88 & 13 \\
\hline & $10-42$ & $8 / 7 / 89$ & 1 & $\mathrm{e}$ & 100 & 10 \\
\hline & $10-43$ & $8 / 7 / 89$ & 1 & $\mathrm{e}$ & 89 & 16 \\
\hline SX-111 & $10-41$ & $8 / 7 / 89$ & 1 & $\mathrm{e}$ & 98 & 11 \\
\hline & $10-42$ & $8 / 7 / 89$ & 1 & $\mathrm{e}$ & 108 & 8 \\
\hline & $10-43$ & $8 / 7 / 89$ & 1 & $\mathrm{e}$ & 98 & 22 \\
\hline SX-112 & $10-41$ & $1 / 7 / 76$ & 1 & $\mathrm{~s}$ & 32 & 2 \\
\hline & $10-42$ & $2 / 5 / 76$ & 2 & $\mathrm{~s}$ & 35 & 9 \\
\hline & $10-43$ & $2 / 5 / 76$ & 1 & $\mathrm{~s}$ & 118 & 8 \\
\hline SX-115 & $10-41$ & $2 / 10 / 75$ & 2 & $\mathrm{~s}$ & 83 & 1 \\
\hline & $10-42$ & $2 / 3 / 75$ & 1 & $\mathrm{~s}$ & 85 & 0 \\
\hline & $10-43$ & $2 / 25 / 75$ & 2 & $\mathrm{~s}$ & 29 & 2 \\
\hline
\end{tabular}

$\mathrm{e}=$ electronically recorded, $\mathrm{s}=$ strip chart digitized by Russ Randall (Three Rivers Scientific) 


\section{Soil Cores}

In addition to the gamma logging data described above, measurements from fifteen core samples associated with SX-108 and SX-115 were incorporated into the composite gamma data set. This data is not available in digital form and thus we were forced to rely exclusively on the 1966 Battelle-Northwest report (Raymond and Shdo, 1966) summarizing soil sampling at SX108 and SX-115. However, the authors only published results from cores recording "abovetrace" Cs-137 concentrations where they defined the "trace" level as $1.0 \mathrm{pCi} / \mathrm{g}$. This included all five soil cores drilled in the vicinity of SX-108 and four cores at SX-115. Cs-137 concentrations from these nine cores were digitized from graphs plotted in Appendix A of Raymond and Shdo (1966). At the six other cores, we assigned "background" level counts (25 type $4 \mathrm{cps)}$ ) at one foot intervals to a depth of 70 feet, the approximate depth to which data was reported from the nine "above-trace" cores. Soil core locations were assigned by triangulation from known coordinates of neighboring vertical drywells using distances estimated from Figures 2 and 3 in Raymond and Shdo (1966). Estimated locations are plotted in Fig. 1. The naming convention used for soil cores in Fig. 1 is entirely our own. Corresponding labels from Figures 2 and 3 in Raymond and Shdo (1966) are included in Table 4.

Table 4. Soil core labels in Fig. 1 and corresponding labels in Raymond and Shdo (1966).

\begin{tabular}{|l|l|l|l|l|l|}
\hline Fig. 1 label & 1966 label & Fig. 1 label & 1966 label & Fig. 1 label & 1966 label \\
\hline $08-50$ & 32A & $15-50$ & $47 \mathrm{~A}$ & $15-55$ & $41 \mathrm{~B}$ \\
\hline $08-51$ & $32 \mathrm{~B}$ & $15-51$ & $48 \mathrm{~B}$ & $15-56$ & $41 \mathrm{~A}$ \\
\hline $08-52$ & 29C & $15-52$ & $48 \mathrm{C}$ & $15-57$ & $45 \mathrm{~A}$ \\
\hline $08-53$ & $29 \mathrm{~A}$ & $15-53$ & $48 \mathrm{~A}$ & $15-58$ & $45 \mathrm{~B}$ \\
\hline $08-54$ & $29 \mathrm{~B}$ & $15-54$ & $49 \mathrm{~A}$ & $15-59$ & $17 \mathrm{~A}$ \\
\hline
\end{tabular}

Soil core Cs-137 concentrations were converted to gross gamma counts in type 4 units using a calibration relationship derived from a scatter plot of historical gamma logging values versus Cs-137 concentrations measured with the SGLS (Fig. 5). Calculation of Cs-137 concentrations from analysis of SGLS spectra is described in the SX Tank Farm Report (DOE, 1996). In creating this plot, we used a methodology identical to that described for the conversion of spectral gamma total counts to type 4 units (Fig. 3). Cs-137 concentrations from vertical drywells in the SX farm were paired with historical gamma values from locations (well/depth) where both types of data were collected. Once again, our search of the historical gamma logging record was restricted to values collected after January 1,1990 . Where location matches could be made, the most recent gross gamma counts (up to a maximum of ten values) were paired with the corresponding Cs -137 concentration. Type 1, 2, and 14 counts were converted to type 4 units as summarized in Table 1 and all historical values were half-life corrected to 1995 values. A linear fit of the resulting scatter plot in log-space (Fig. 5) yields the conversion:

$$
\text { type } 4 \mathrm{cps}=\exp \{3.5709+0.4080 * \log (\mathrm{Cs}-137 \mathrm{pCi} / \mathrm{g})\}
$$

After conversion to type 4 counts, all soil core data was half-life corrected to 1995 values. 


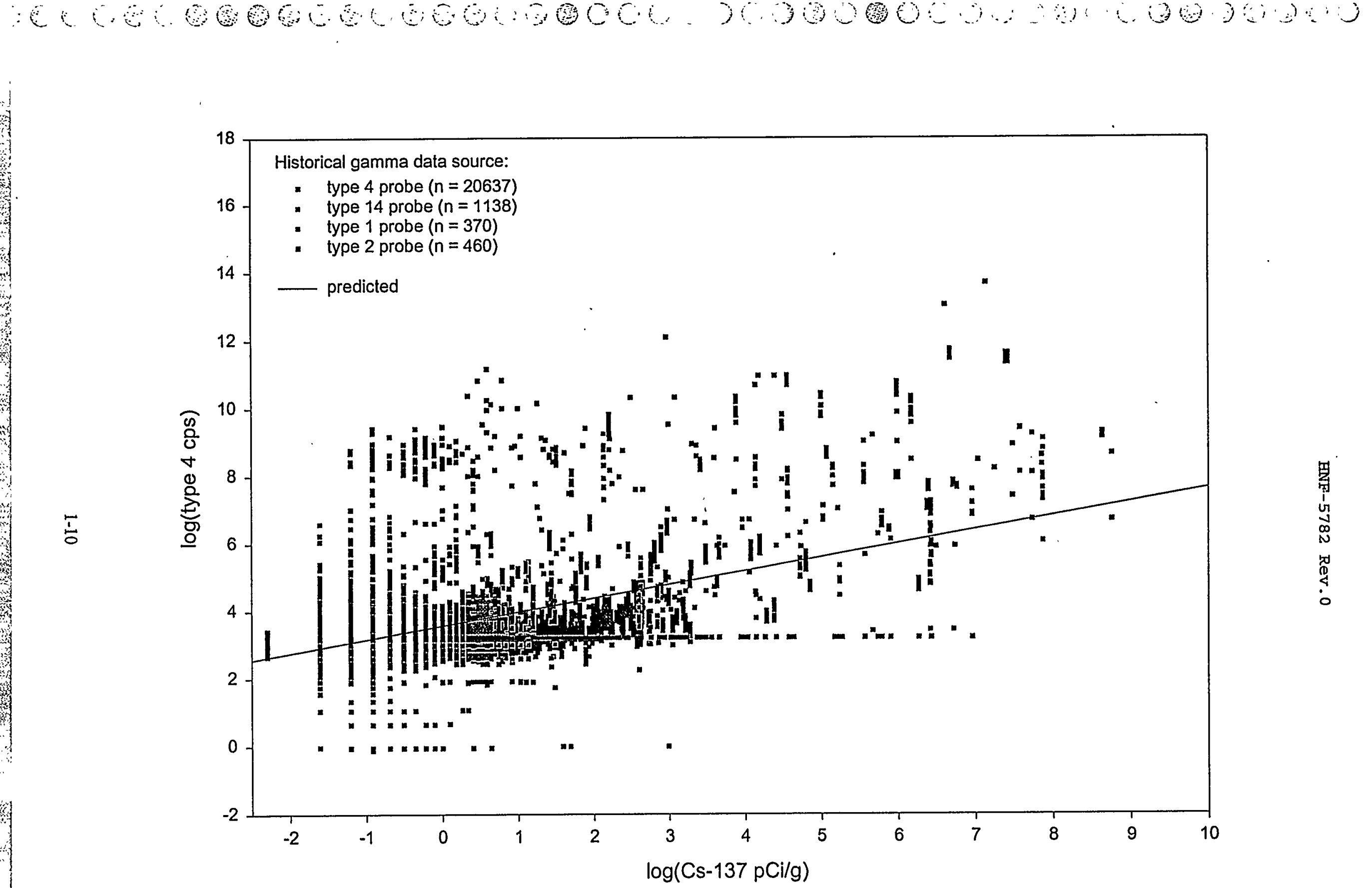

Fig. 5. Historical gamma logging values vs. Cs-137 concentrations measured with the spectral gamma detector. 


\section{Histograms}

Histograms of all values in the composite gamma data set and broken down among five different detectors used at the SX tank farm are plotted in Fig. 6. The distribution of all gamma logging values following conversion to consistent units is highly left-skewed. Most of these values $(18,635$ out of 22,616 total) were recorded with the spectral gamma detector over low to intermediate levels of contamination (gross gamma counts between 1.0 and 12.0 in log-space). The LVMS, type 1 , and type 4 probes logged counts over a similar range. At intermediate and high levels of contamination, the type 2 probe recorded the majority of values, logging all counts in the composite data set greater than 13.0. Note that the conversion-to-"background" of 192 zero values recorded with the type 2 probe within lateral drywells caused the spike in type 2 counts between 3.0 and 4.0. Conversion of lateral zeroes did not have as pronounced an effect in the histogram of type 1 counts where 145 zeroes were set to "background."
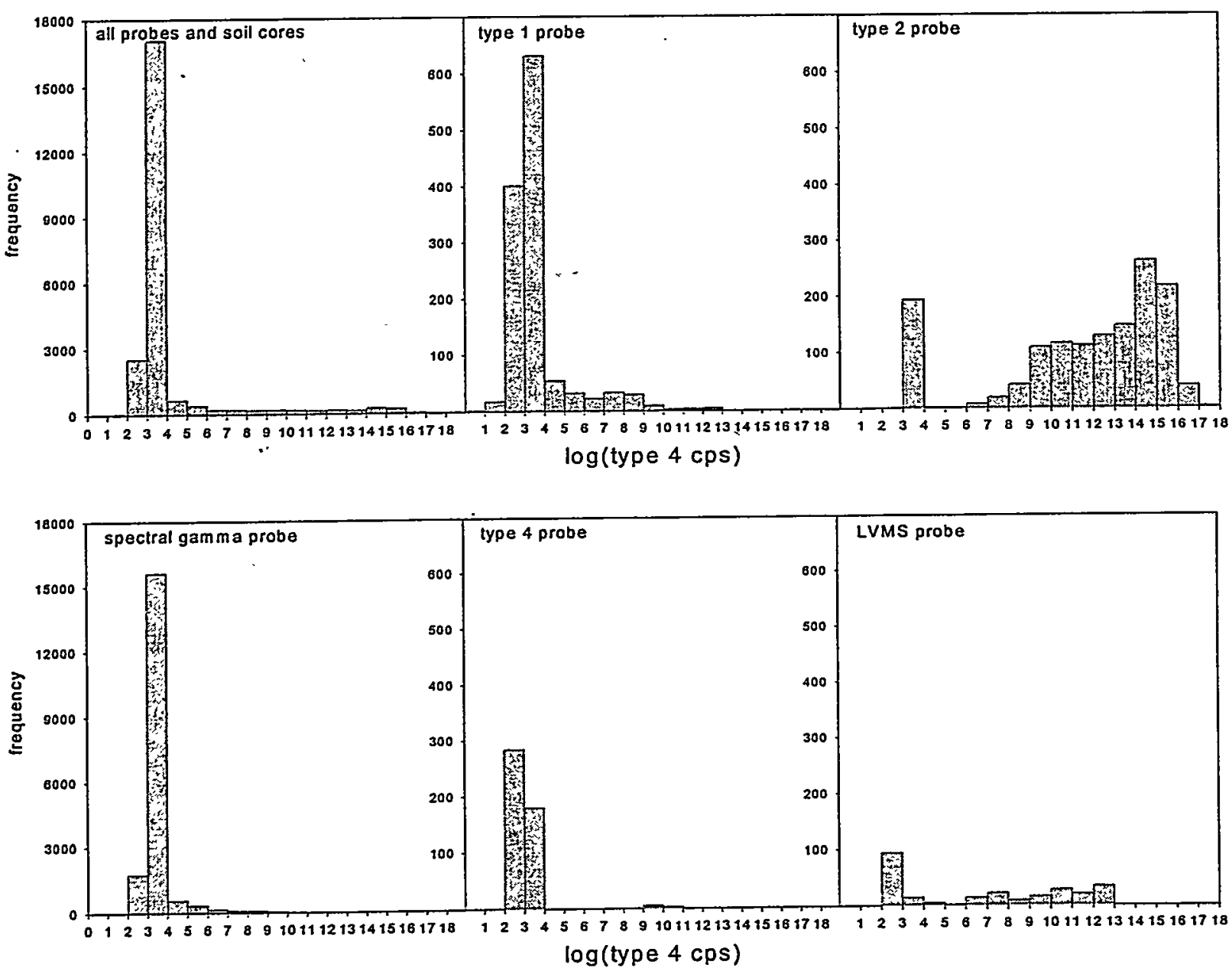

Fig. 6. Histograms of all values in the composite SX data set after conversion to consistent units and broken down by probe type. 
The histogram of all gamma logging values less than $100 \mathrm{cps}$ (Fig. 7) illustrates the distribution of "background"-level counts. This histogram supports the definition of a nominal "above-background" threshold of 4.0 in log-space (approximately $55 \mathrm{cps}$ untransformed). Within the full composite data set, 19,611 values (out of 22,616 ) fall below this threshold. Thus, approximately $87 \%$ of the data is nominally "clean."

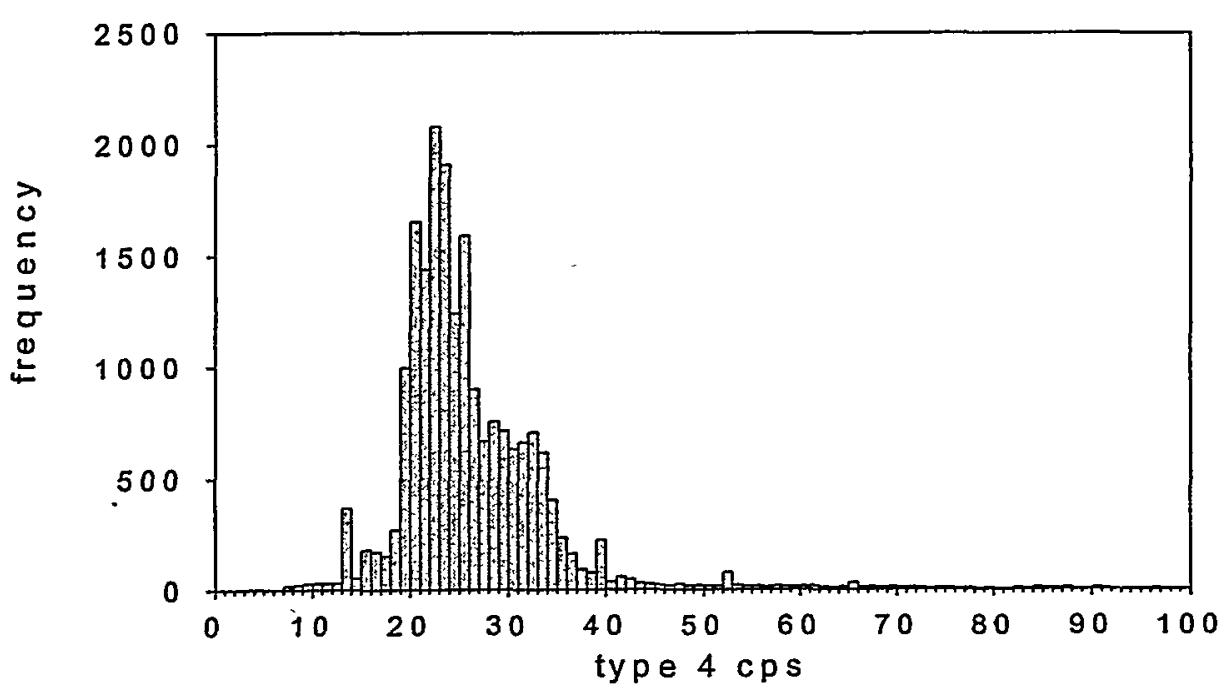

Fig. 7. Histogram of all values less than $100 \mathrm{cps}$ after conversion to consistent units.

Histograms of gamma counts within vertical and lateral drywells are plotted in Fig. 8 and Fig. 9, respectively. Compared to the complete composite gamma data set (Fig. 6), the distribution of all vertical drywell counts (Fig. 8) is similarly skewed but is not as flat at "abovebackground" levels of contamination. Lateral gamma logging values are bimodally distributed with $50 \%$ of all lateral counts greater than 4.0 in log-space (Fig. 9), revealing the importance of lateral data in defining "above-background" regions within the prediction volume.

The type 4, LVMS, and spectral gamma detectors were only used in vertical drywells and thus the histograms of vertical drywell counts from these probes (Fig. 8) are identical to those plotted in Fig. 6 . Only three values from the type 14 probe were included in the data set and thus a type 14 histogram is not plotted. Recall that values from all probes other than the spectral gamma detector represent patches where the spectral gamma detector saturated, exceeded deadtime limits, or was not used. Type 1 counts cluster at intermediate values. Note that relatively few type 2 values define the highest levels of contamination measured in vertical drywells. Only 64 type 2 values are greater than 13.0 in log-space.

Within lateral drywells, type 1 data defines low and intermediate levels of contamination while type 2 counts are skewed toward intermediate and high values (Fig. 9). As noted above, the frequency of values between 3.0 and 4.0 in all three histograms in Fig. 9 reflects the conversion of zero values to the nominal "background" of $25 \mathrm{cps}$. 

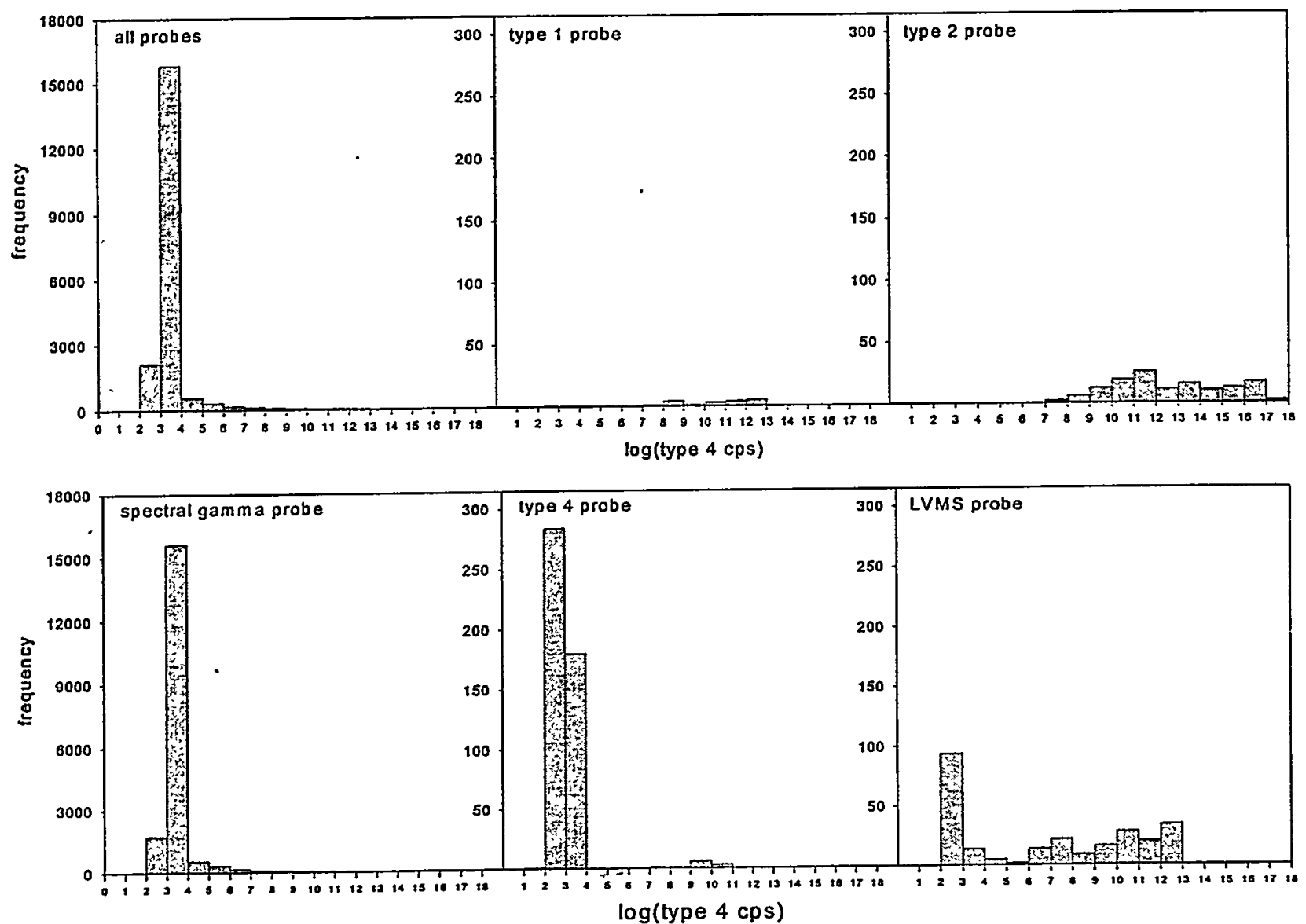

Fig. 8. Histograms of vertical drywell gamma counts recorded with five probe types. All values converted to consistent units.

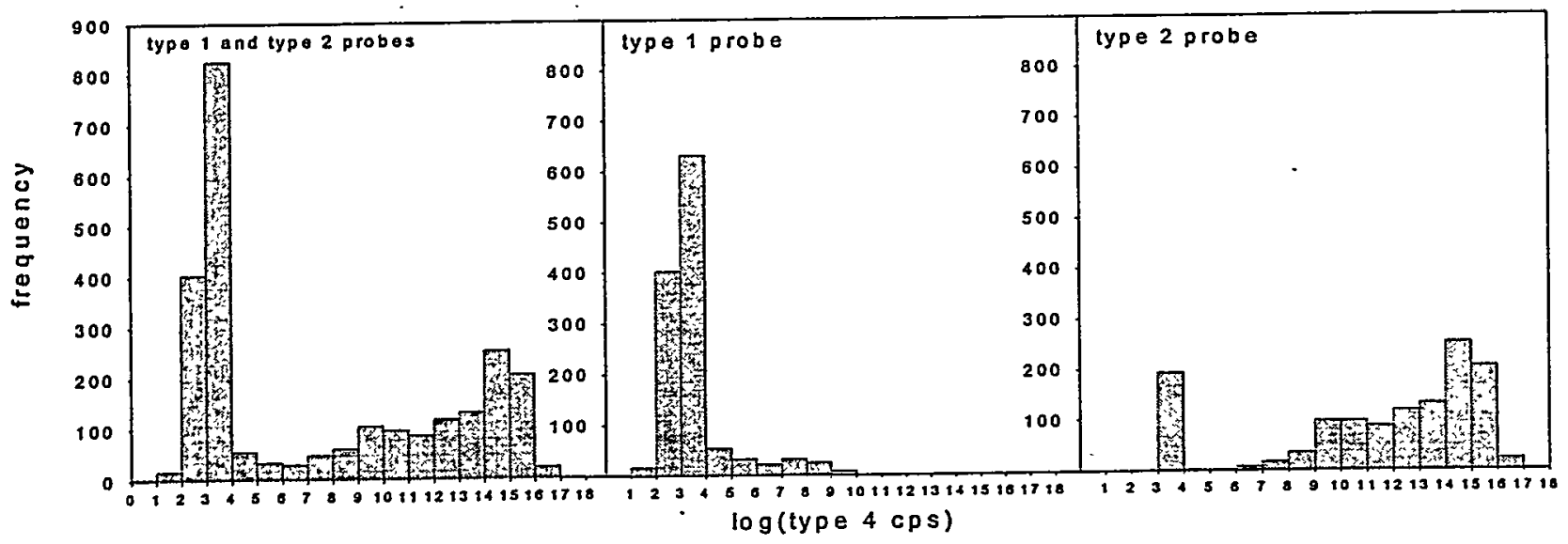

Fig. 9. Histograms of lateral gamma counts recorded with type 1 and type 2 probes. All values converted to consistent units. 
The distribution of maximum gamma counts by well among vertical and lateral wells also shows the dominance of lateral data at "above-background" levels of contamination (Fig. 10). All but one lateral out of 24 recorded a maximum value greater than 4.0 in log-space while 16 out of 99 vertical drywells logged maximums between 3.0 and 4.0. Note, as well, that 74 out of 99 vertical drywells recorded maximums between 3.0 and 6.0. The relatively modest levels of contamination found at most vertical drywells proved to be important in geostatistically defining the horizontal extent of contaminated plumes found at the SX farm. However, the importance of vertical wells in intercepting regions of high contamination should not be understated. At very high levels of contamination (between 12.0 and 18.0) equal numbers of vertical and lateral wells (10) recorded maximum values.


Fig. 10. Distribution of maximum counts per well recorded at vertical and lateral drywells.

The distribution of soil core data after conversion to consistent units is plotted in Fig. 11, revealing an intermediate level of contamination among the nine soil cores originally recording "above-trace" concentrations of Cs-137. The relatively large number of values between 3.0 and 4.0 in log-space (420 total) is the result of assigning "background" level counts at one foot intervals (70 per core) to the six cores where no "above-trace" Cs- 137 concentrations were observed. The distribution of maximum values per core reflects this assignment of "background" level counts to six cores. The other maximums, once again, reveal an intermediate level of contamination with no maximum greater than 13.0. 

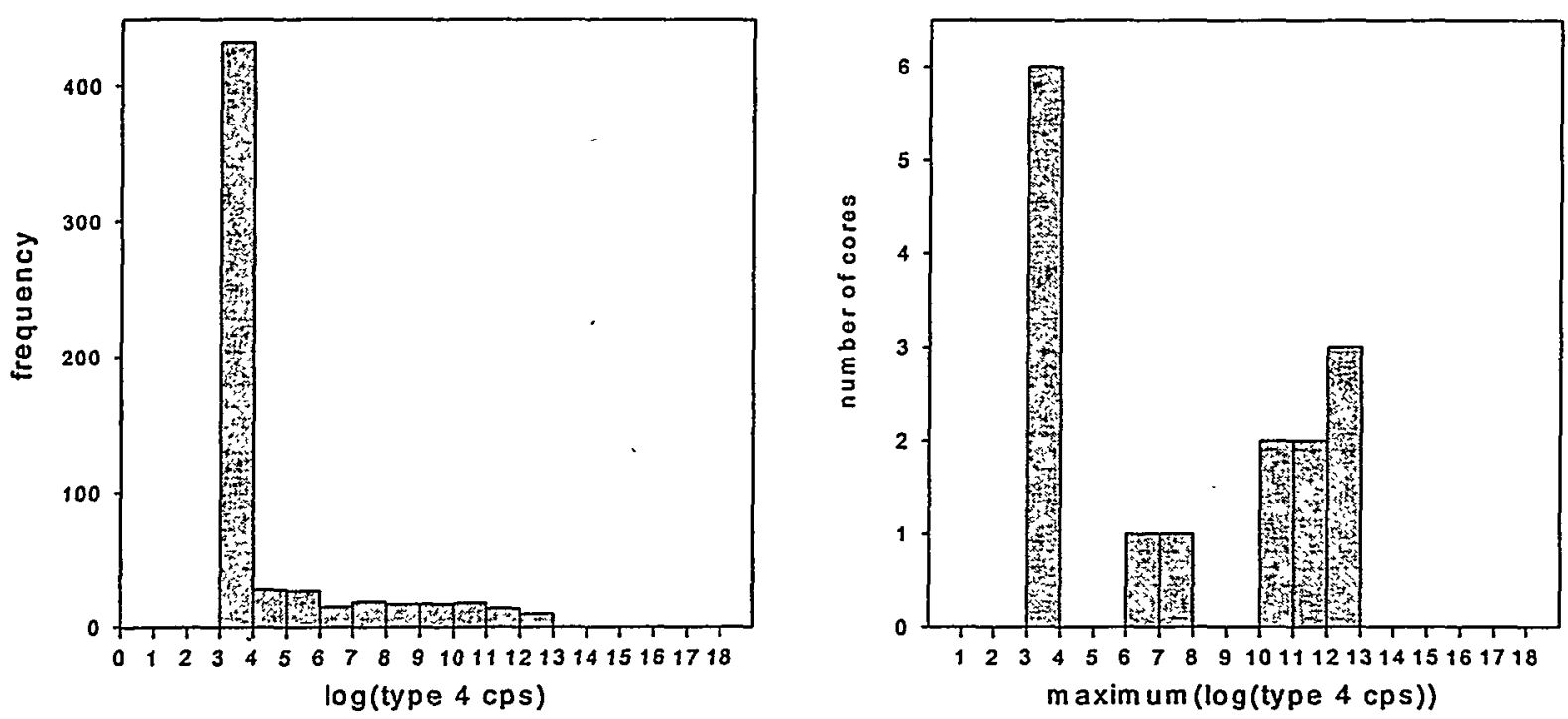

Fig. 11. Histogram of soil core values after conversion to consistent units and distribution of maximum values per core recorded at soil cores.

\section{Data binning}

In vertical and lateral drywells, gamma counts were recorded at short distance intervals. Vertical drywell data was typically spaced at $0.5 \mathrm{ft}$. intervals while lateral counts were generally recorded at one $\mathrm{ft}$. spacings. In reducing the size of the composite gamma data set $(\mathrm{n}=22,616)$ to a more computationally tractable size, we used a binning procedure based on a 1-meter grid system established with vertex $(0,0,0)$ located at easting $566745 \mathrm{~m}$, northing $134145 \mathrm{~m}$, and the surface of the prediction volume. During binning, each data value was assigned to the nearest grid point in the prediction volume. The location of the center of mass and the mean of the logtransformed values in each non-empty bin $(n=3,957)$ were used as input in geostatistical analyses.

Histograms of binned data are plotted in Figure 12. Qualitatively, the binned distributions of all data and vertical drywell data closely resemble their unbinned analogues (Figs. 6 and 8). Relative to the distribution of unbinned lateral counts (Fig. 9), the histogram of binned lateral data is slightly flattened in the neighborhood of the upper mode between 14 and 15 in log-space. This result is not unexpected given the spatial averaging implicit in our binning method. However, the overall similarity between binned and unbinned distributions of the composite data set suggests that binning is an appropriate method for reducing the size of the data set. 

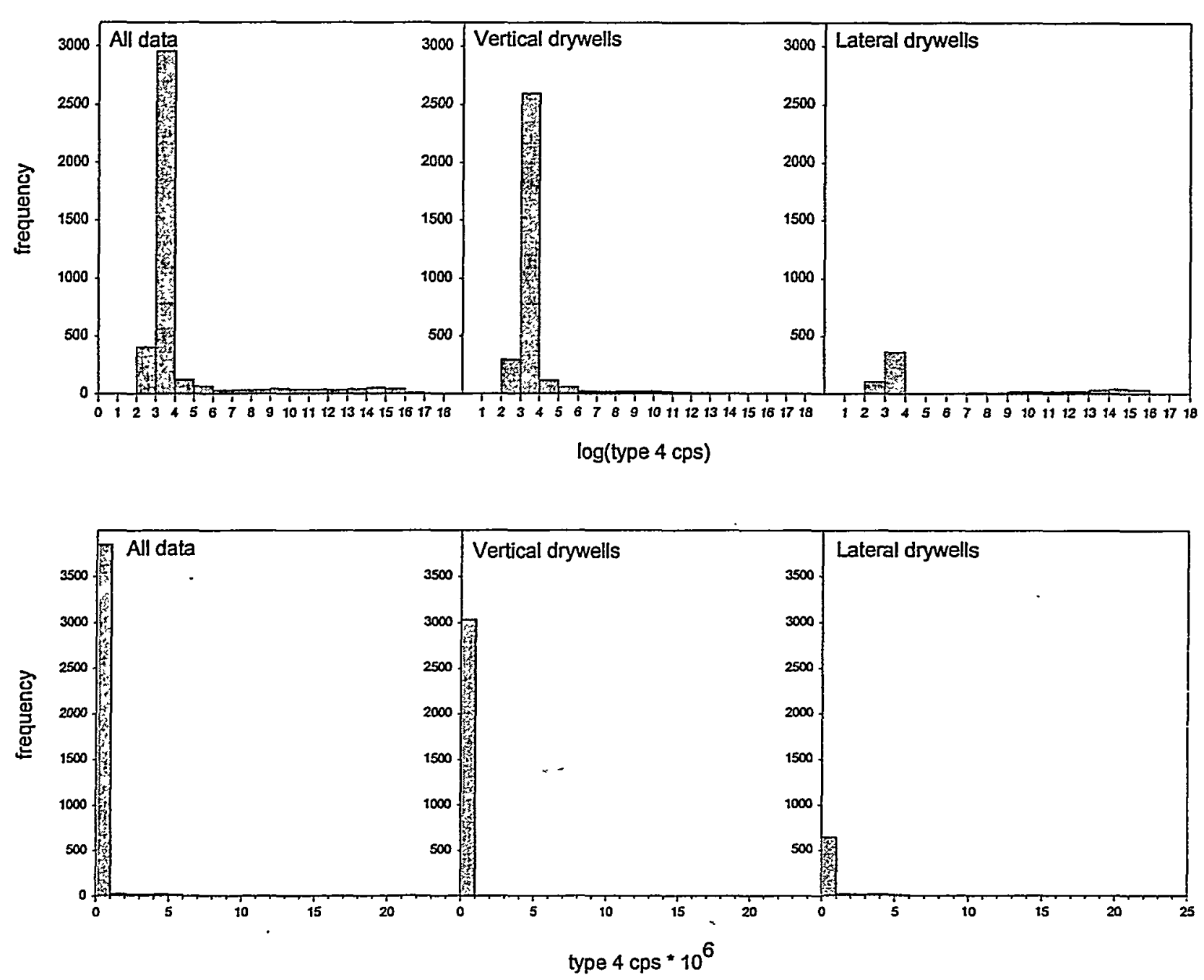

Fig. 12. Histograms of vertical, lateral, and all gamma logging values after conversion to consistent units and binning by 1 meter cubes. 


\section{The composite $\mathrm{Cs-137}$ data set}

The Cs-137 data set is a composite of $\mathrm{Cs}-137$ concentrations in $\mathrm{pCi}^{-1}$ including values derived from the analysis of SGLS spectra at vertical drywells, values predicted from spectral gamma and historical gamma counts logged at both vertical and lateral drywells, and Cs-137 concentrations measured directly from soil cores collected in the SX-108, SX-115 neighborhood.

\section{.Spectral gamma Cs-137 gaps}

While spectral gamma Cs-137 concentrations were calculated from the same logging runs at vertical drywells where spectral gamma total counts were recorded (DOE, 1996; DOE, 1997; Brodeur, 1996), fewer Cs-137 concentrations were reported by Mactec-ERS - 7,721 values over the entire SX farm versus 18,635 spectral gamma total counts over the entire SX farm. A histogram of spectral gamma total counts logged at locations (well/depth) where no Cs-137 concentrations were reported by Mactec-ERS is plotted in Fig. 13. Approximately $99 \%$ of these values are less than 6.0 in log-space, suggesting that gaps in the spectral Cs-137 data are concentrated in regions of "background-level" contamination. Additional gaps in the spectral gamma Cs-137 data due to detector saturation, high dead times, or other reasons are identical to gaps in the spectral gamma total counts data summarized in Table 2. At drywell 09-39 we did not use LVMS data over the entire well, but only over the interval where the spectral gamma detector saturated (62-90 ft.).

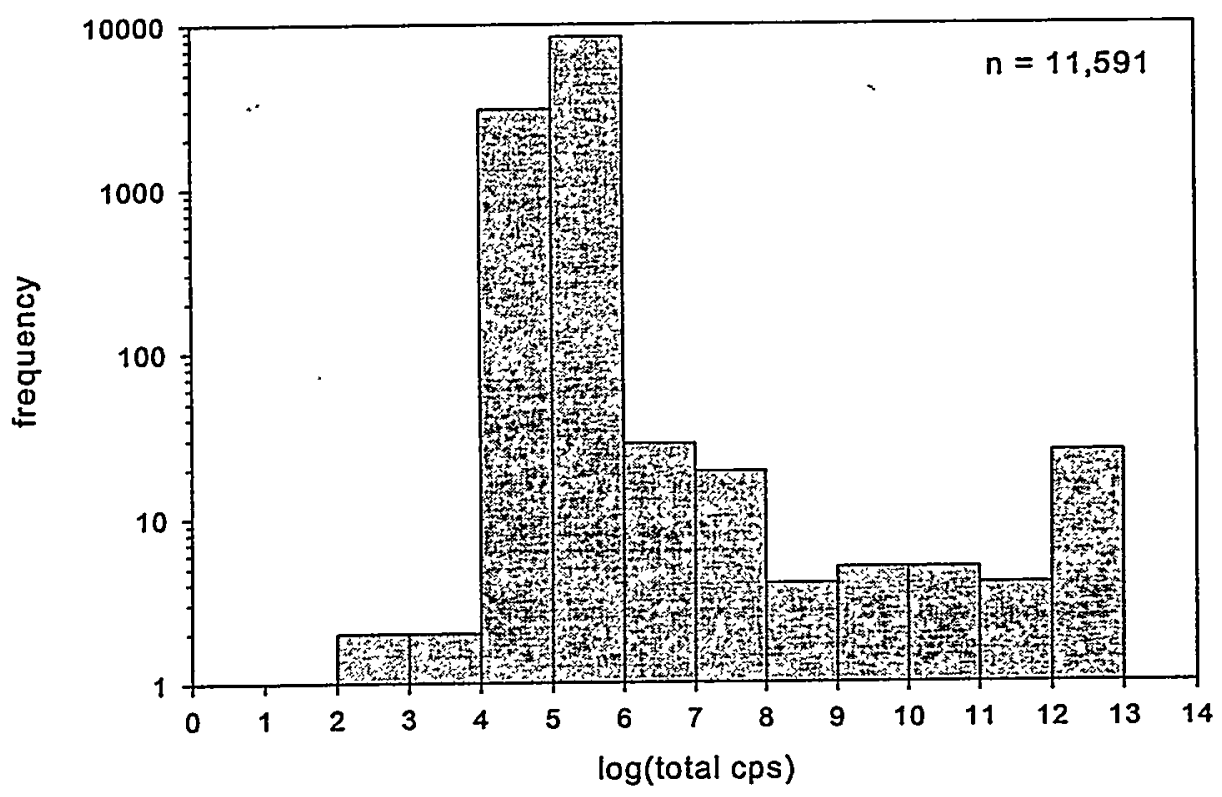

Fig. 13. Spectral gamma total counts recorded at locations (well/depth) in the SX farm where no spectral gamma Cs-137 concentration data was recorded 


\section{Vertical drywells, lateral drywells, and soil cores}

In filling gaps in the vertical drywell data, we converted gross gamma counts recorded with either the spectral or historical gamma detectors to $\mathrm{Cs}-137$ concentrations in $\mathrm{pCi} \mathrm{g}^{-1}$. The basis for the conversion of spectral gamma counts was a scatter plot of spectral gamma Cs-137 concentrations versus spectral gamma total counts recorded at identical locations, i.e. the same well and depth. Note that in comparing well depths, depth values originally reported by MactecERS to the nearest one-hundreth of a foot were rounded to the nearest tenth. Following logtransformation of both variables, the resulting scatter plot was fit with a power curve (Fig. 14).

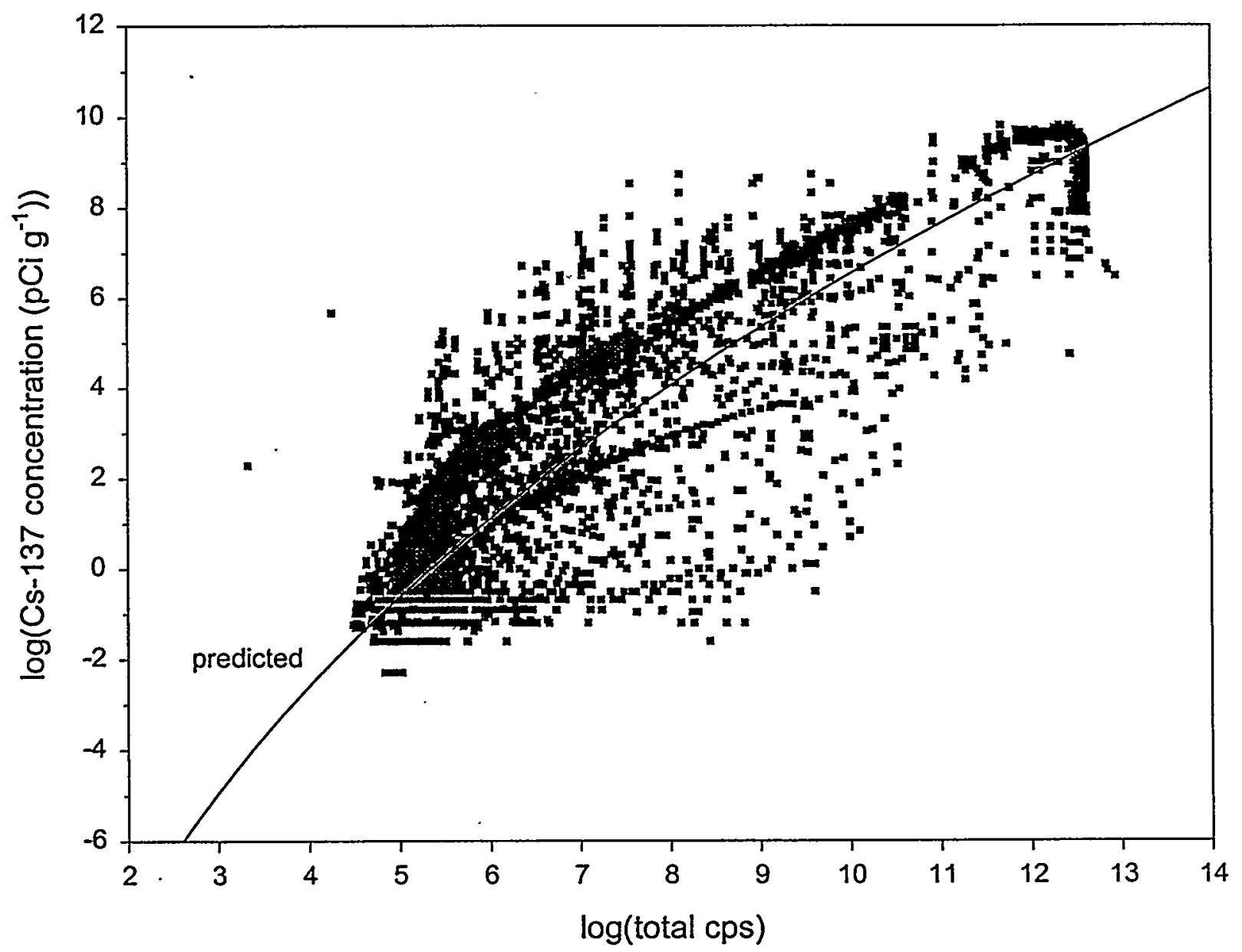

Fig. 14. Spectral gamma Cs-137 $\mathrm{pCi} \mathrm{g}^{-1}$ vs. spectral gamma total counts. 
A similar scatter plot was used to derive a calibration relationship between gamma counts in probe type 4 units and Cs-137 concentrations. In this case, spectral gamma total counts were first converted to type 4 units, as described in Table 1, before they were log-transformed and plotted against the log of spectral gamma Cs-137 concentrations (Fig. 15). Note that the . maximum value in type 4 units among these pairs (the blue squares in Fig. 15) is approximately $\mathrm{e}^{10} \mathrm{cps}$. However, recall from the histograms of lateral gamma counts (Fig. 9), that a significant number of lateral gamma counts are greater than $\mathrm{e}^{10}$ in type $4 \mathrm{cps}$. Given that the laterals appear to be intercepting the most highly contaminated regions of the SX-farm, we were uncomfortable merely fitting a line to the paired spectral data and using that relationship to predict Cs-137 concentrations from lateral counts of magnitudes beyond the range of the given scatter plot. Our solution was to use the composite gamma counts data set minus the soil core data to make kriging predictions of $\log$ (type $4 \mathrm{cps}$ ) at locations where SX-108 soil core data was collected. The resulting pairs of soil core Cs-137 concentrations in $\log \left(\mathrm{pCi}^{-1}\right)$ versus $\log$ (predicted type 4 cps) are plotted with red squares in Fig. 15. A straight line conversion was fit to the combined scatter plot (Fig. 15).

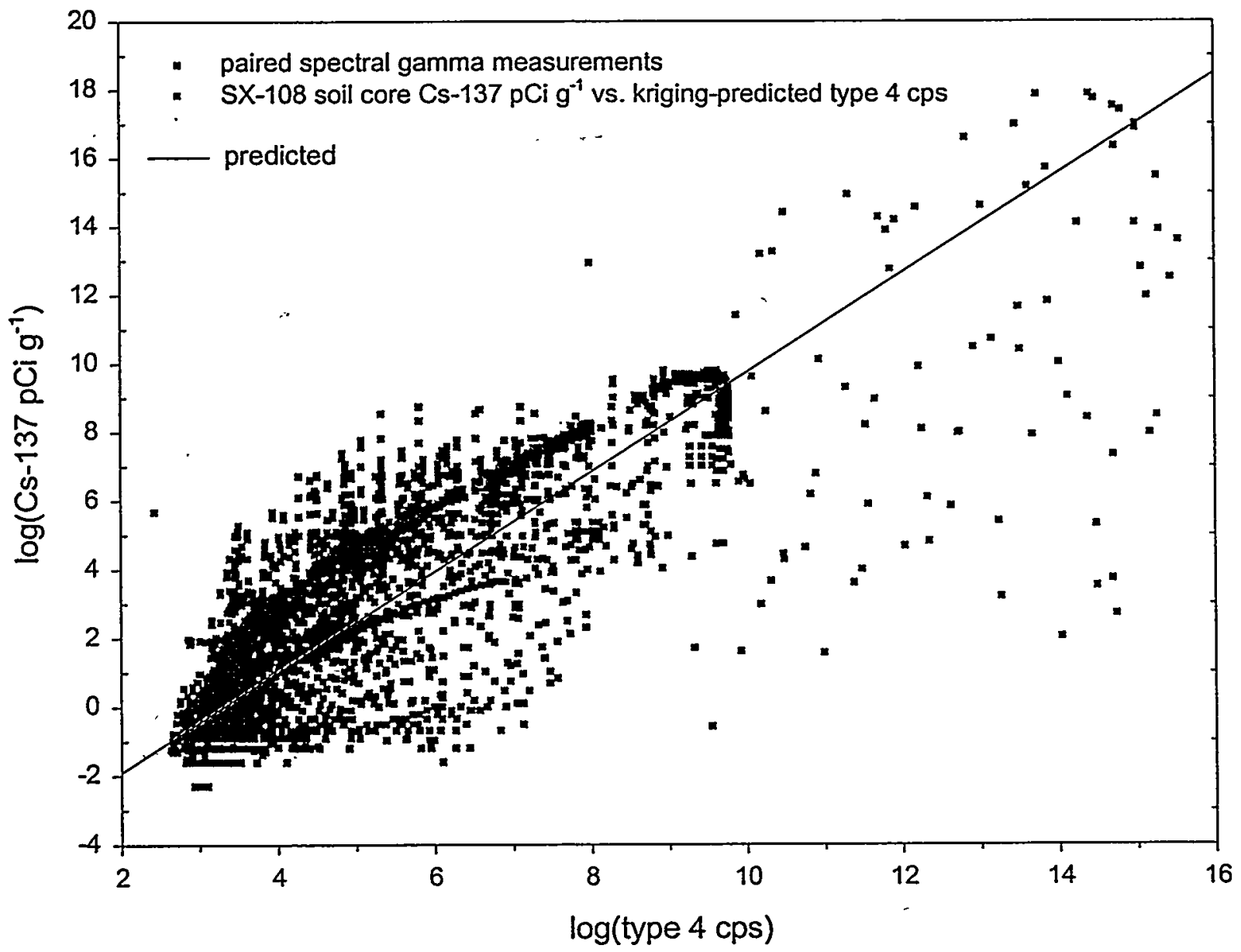

Fig. 15. Spectral gamma Cs-137 $\mathrm{pCi} \mathrm{g}^{-1}$ vs. spectral gamma total counts in type 4 units and SX-108 soil core Cs-137 $\mathrm{pCi}^{-1}$ vs. kriging-predicted type 4 cps. 
Note that results from the ten soil cores collected in the vicinity of SX-115 were not included in generating the conversion from historical gamma logging to Cs-137 concentrations. A plot of $\log \left(\mathrm{pCi}^{-1}\right)$ versus $\log$ (predicted type $4 \mathrm{cps}$ ) from SX-115 soil cores (Fig. 16) reveals little correspondence between measured Cs-137 concentrations and kriging-predicted counts. At SX-115, the data do not appear to support the kriging model. Over a "background" range of predicted counts, $\mathrm{e}^{3}$ to $\mathrm{e}^{4} \mathrm{cps}$, measured Cs-137 concentrations range as high as $\mathrm{e}^{18} \mathrm{pCi} \mathrm{g}^{-1}$ (Fig. 16). Where a line of points is seen parallel to the $x$-axis of Fig. 16, counts slightly above "background" were predicted at soil cores originally assigned a "background" level of contamination. Given these inconsistencies, we chose to not include SX-115 values in Fig. 15.

Conversions from gross gamma counts in either spectral gamma or type 4 units to Cs-137 $\mathrm{pCi} \mathrm{g}^{\mathrm{l}}$ are summarized in Table 5. Using the spectral gamma counts-to-pCi $\mathrm{g}^{-1}$ conversion, spectral gamma total counts filling gaps in the vertical drywell Cs-137 data, i.e. the values represented in Fig. 13, were converted to Cs-137 concentrations and added to the composite Cs137 data set. Similarly, the probe type 1,2, 4, and 14 historical gamma counts previously used to patch spectral gamma gaps in forming the composite gamma counts data set (Table 2), were converted to $\mathrm{Cs}-137$ concentrations using the type 4 counts-to-pCi $\mathrm{g}^{-1}$ conversion. All lateral drywell gamma counts from the composite gamma counts data set were converted to Cs-137 concentrations using the type 4 counts-to- $\mathrm{pCi}^{-1}$ conversion. The six soil cores previously assigned "background" level gamma counts were assigned Cs-137 concentrations using the same conversion while the nine soil cores recording "above-trace" Cs-137 concentrations were added to the composite Cs-137 data set without modification.

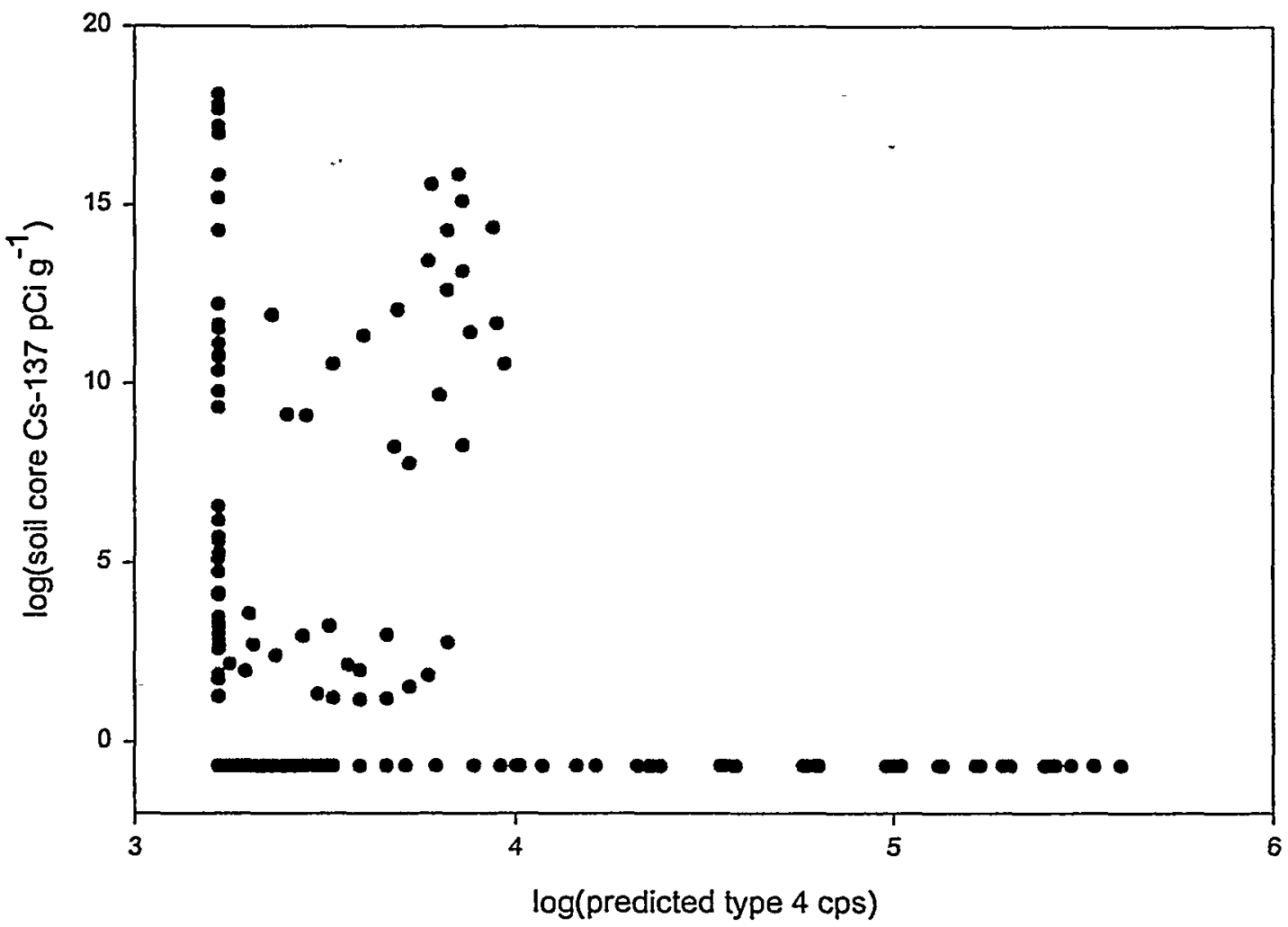

Fig. 16. SX-115 soil core Cs-137 $\mathrm{pCi}^{-1}$ vs. kriging-predicted type 4 cps. 
Table 5. Conversion of gross gamma counts in spectral gamma or type 4 units to Cs- $137 \mathrm{pCi} \mathrm{g}^{-1}$.

\begin{tabular}{|l|l|}
\hline Gamma counts (units) & Conversion to $\mathbf{C s - 1 3 7} \mathbf{p C i ~}^{-1}$ \\
\hline spectral gamma & $\exp \left\{-28.5363+16.4561 *(\log (\text { spectral gamma cps }))^{0.3287}\right\}$ \\
\hline type 4 & $\exp \{-4.7939+1.4547 * \log ($ type $4 \mathrm{cps})\}$ \\
\hline
\end{tabular}

\section{Histograms}

Histograms of all values in the composite Cs-137 data set and broken down by probe type are plotted in Fig. 17. Similar to the composite counts data set, the distribution of all values is left-skewed, even after log-transformation. The distribution of spectral gamma pCi $\mathrm{g}^{-1}$ spans intermediate levels of contamination, while spectral gamma counts converted to $\mathrm{pCi} \mathrm{g}^{-1}$ are centered around "background" Cs-137 activities. While Cs-137 concentrations derived from type 1 and 2 counts are fewer in numbers, note their importance in defining the highest levels of contamination. The distribution of soil core values is fairly uniform between 1.0 and 18.0 in logspace, with the spike between 0.0 and 1.0 the result of assigning "background" activities to six cores. Type 4; 14, and LVMS values are bimodally distributed, with one mode at "background" and a smaller mode between $\mathrm{e}^{12}$ and $\mathrm{e}^{13} \mathrm{pCi} \mathrm{g}^{-1}$.
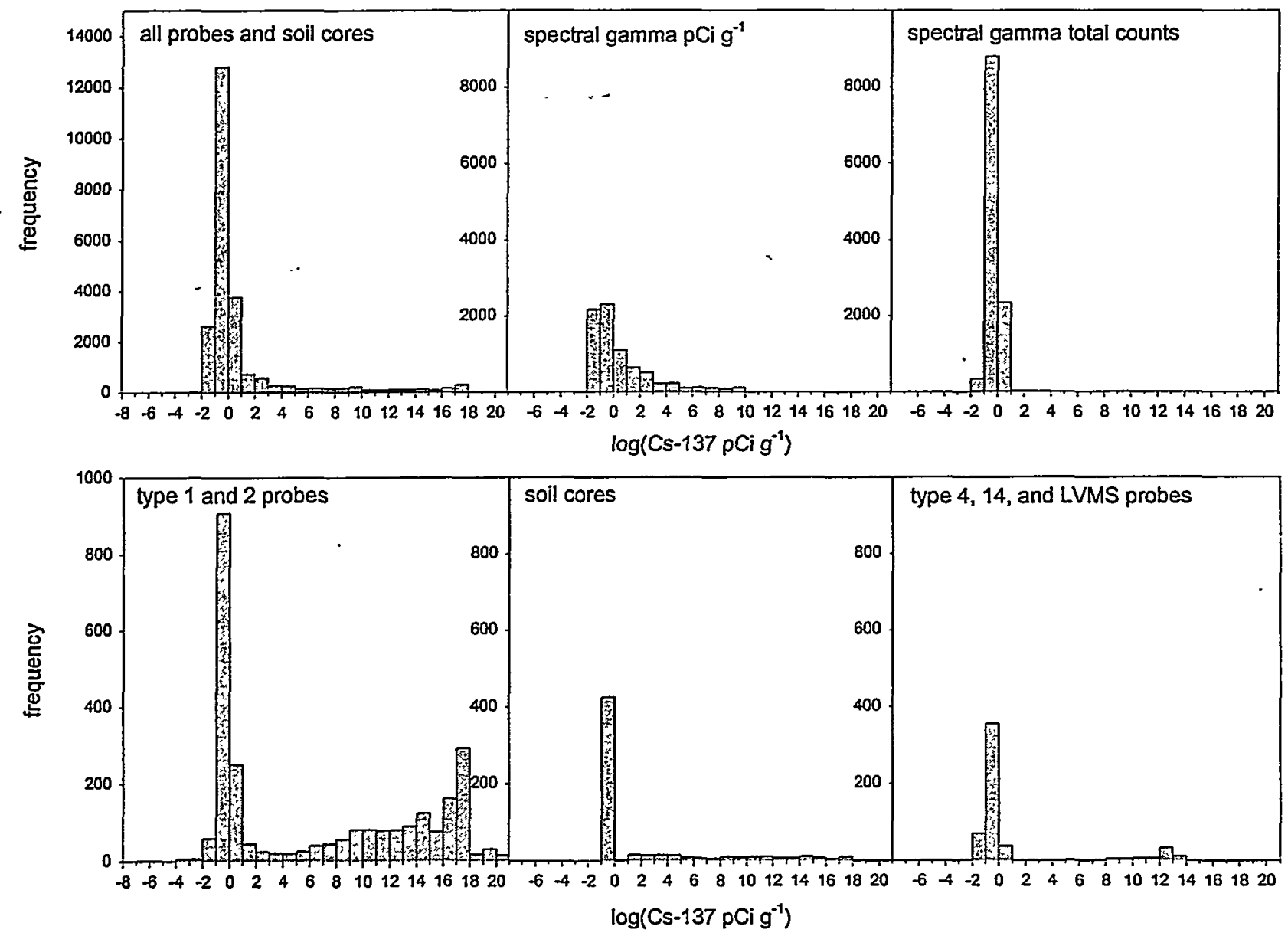

Fig. 17. Histograms of all values in the composite Cs-137 data set after conversion to $\mathrm{pCi} \mathrm{g}^{-1}$ and broken down by probe type. 
Histograms of Cs-137 concentrations measured at vertical drywells are plotted in Fig. 18. Given that the spectral gamma detector was used only in vertical drywells, the spectral gamma distributions in Fig. 18 are identical to those plotted in Fig. 17. Once again, note that a relatively small number of type 2-derived values define the highest Cs-137 activities measured in vertical drywells.
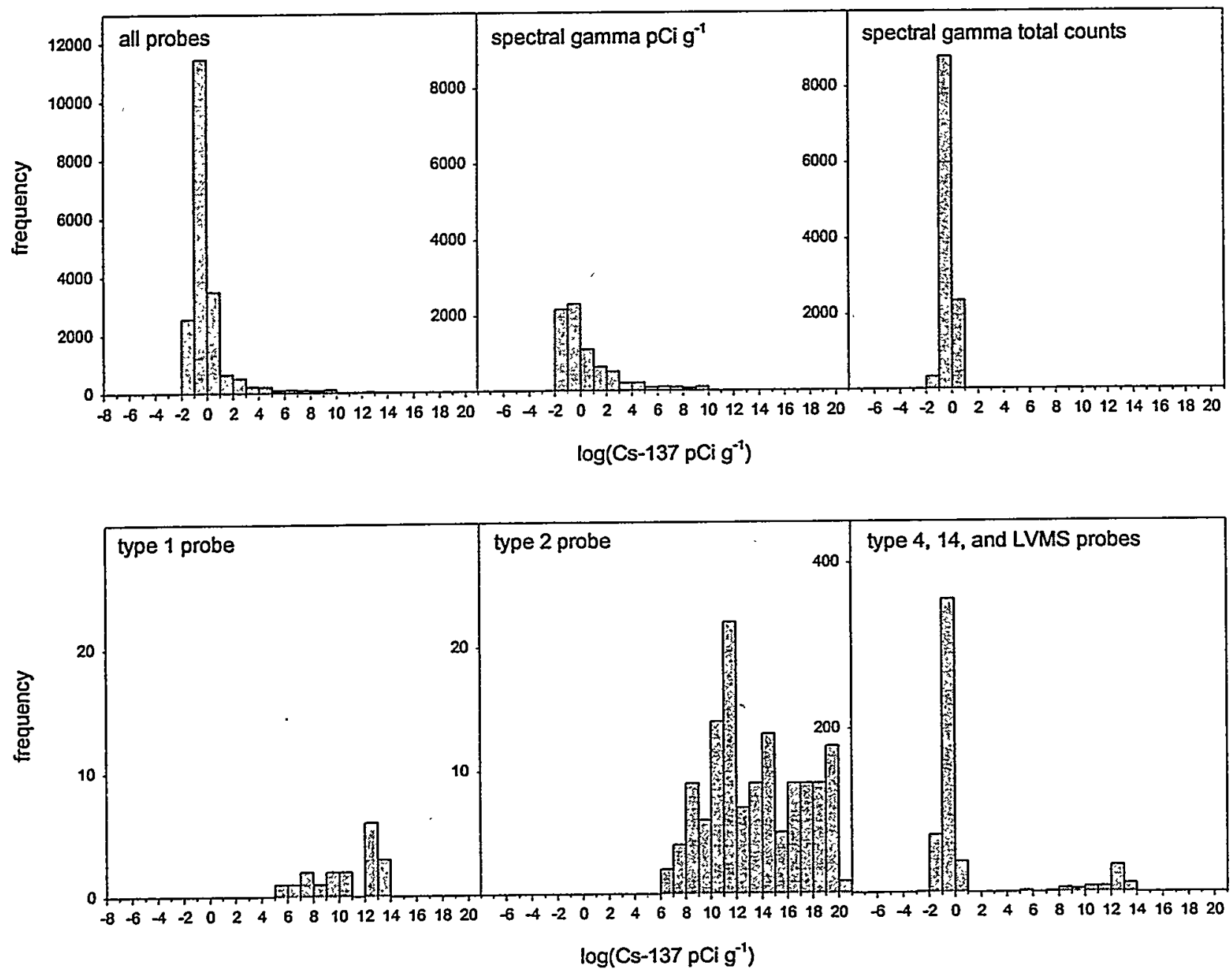

Fig. 18. Histograms of vertical drywell values in the composite Cs-137 data set after conversion to $\mathrm{pCi} \mathrm{g}^{-1}$ and broken down by probe type.

Histograms of Cs-137 concentrations derived from conversion of type 1 and 2 counts to $\mathrm{pCi}^{-1}$ in lateral drywells are plotted in Fig. 19. Type 1 values span low to intermediate Cs-137 concentrations while type 2 values dominate the intermediate to high range of Cs-137 concentrations. Recall that the type 2 peak between $\mathrm{e}^{-1}$ and $\mathrm{e}^{0} \mathrm{pCi}^{-1}$ is the result of assigning "background" activities to zeroes logged by the type 2 probe (see Table 3 ). 


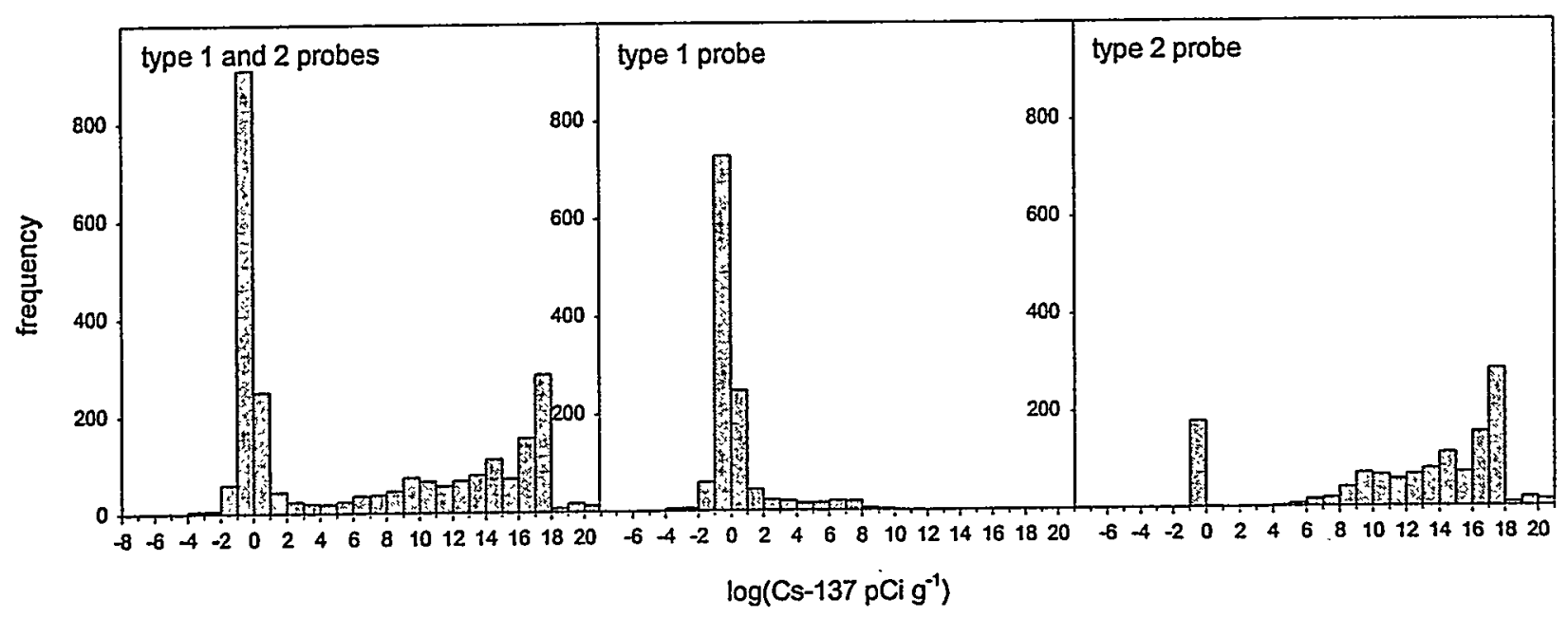

Fig. 19. Histograms of lateral drywell values in the composite Cs-137 data set after conversion to $\mathrm{pCi} \mathrm{g}^{-1}$ and broken down by probe type.

\section{Binning}

The same binning methodology described earlier was used to reduce the size of the composite Cs-137 data set from 23,072 values to a more computationally tractable size of 3,962 values. A histogram of the binned Cs-137 data is plotted in Fig. 20. Where bins contained more than one value, a bin sample variance was calculated. Histograms of bin sample variance and bin sample number (number of values per bin) are also plotted in Fig. 20.

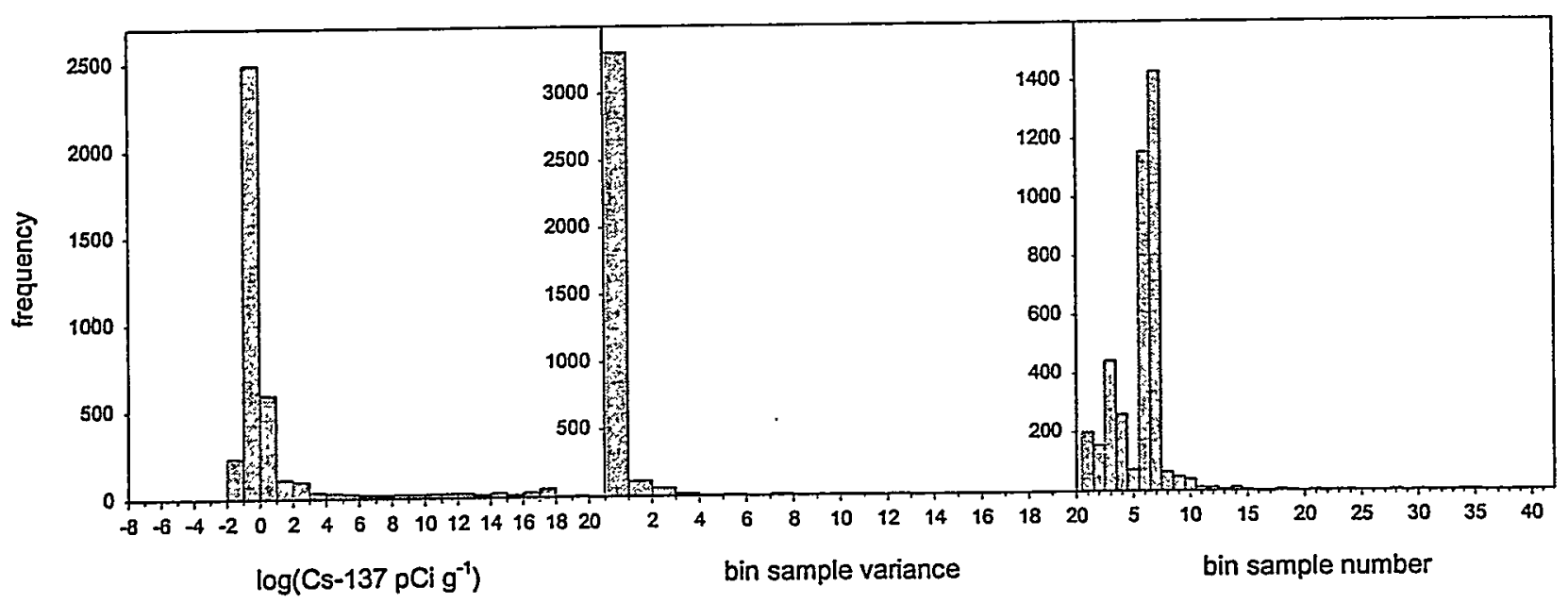

Fig. 20. Histograms of the binned composite Cs-137 data set, bin sample variance and bin sample number. 


\section{Drywell 09-39}

An opportunity to assess the accuracy of our counts-to-pCi $\mathrm{g}^{-1}$ conversions presents itself at drywell 09-39. Recall that well 09-39 was drilled in December, 1996 and logged with both spectral gamma and LVMS detectors (DOE, 1997). Additionally, a limited number of soil.core samples were collected during drilling of the well (Dave Meyers, pers. comm., 11/15/99). A vertical profile of Cs-137 concentrations at well 09-39 is plotted in Fig. 21. Spectral gamma counts converted to $\mathrm{pCi}^{-1}$ are plotted only at depths where no spectral gamma Cs-137 measurements were recorded. LVMS counts were treated as equivalent to type 4 counts as discussed above (see Fig. 4), before conversion to $\mathrm{pCi}^{-1}$. LVMS-derived Cs-137 concentrations are plotted only at depths where the spectral gamma detector saturated.

Below a depth of $50 \mathrm{ft}$., the overall pattern of soil core values closely resembles the more continuous profile generated from drywell measurements (Fig. 21). However, soil core values are consistently greater than or equal to $\mathrm{Cs}-137$ concentrations derived from gamma logging, suggesting that drywell measurements may be consistently underestimating the true Cs-137 concentration in the vadose zone. At $65.5,74.5$, and $79.5 \mathrm{ft}$. below surface, our type 4 cps-to$\mathrm{pCi}^{-1}$ conversion generates values approximately an order-of-magnitude smaller than soil core Cs-137 concentrations. At depths of $56.5,102.5$, and $108.5 \mathrm{ft}$, spectral gamma Cs-137 concentrations appear to underestimate true values by approximately, three, two, and one order(s)-of-magnitude, respectively. Note that Cs-137 concentrations derived from our spectral gamma cps-to-pCi g ${ }^{-1}$ conversion at depths of 90 and $103 \mathrm{ft}$ correspond closely to neighboring spectral gamma $\mathrm{pCi}^{-1}$, suggesting that this conversion may also underestimate $\mathrm{Cs}-137$ concentrations.

Consistent underestimation of $\mathrm{Cs}-137$ concentrations may be the result of instrumentation operating at performance limits. While the spectral gamma detector was saturated from 62 to 68 $\mathrm{ft}$. and from 70 to $90 \mathrm{ft}$., assays from 90 to $110 \mathrm{ft}$. were deemed questionable because many of the spectra recorded over this interval were highly distorted (DOE, 1997). Similarly, the authors of the well 09-39 report (DOE, 1997) concluded that the LVMS detector was saturated from 63 to $68 \mathrm{ft}$. and from 72 to $85 \mathrm{ft}$. Aside from instrument shortcomings, it also seems unlikely that our type $4 \mathrm{cps}$-to-pCi $\mathrm{g}^{-1}$ conversion would tend to underestimate $\mathrm{Cs}-137$ activities at high count rates. Note in Fig. 15, that soil core Cs-137 activities given predicted type $4 \mathrm{cps}$ (the red squares) tend to fall approximately two log cycles below the regression line, implying that our regression conversion is overestimating $\mathrm{pCi} \mathrm{g}^{-1}$ when we apply it in the range of high count rate, type 2 data.

We cannot explain the three orders-of-magnitude discrepancy between spectral gamma counts converted to $\mathrm{pCi} \mathrm{g}^{-1}$ and soil core measurements at 25.5 and $44.5 \mathrm{ft}$. However, it seems logical to assume that the reported soil core values are erroneous given that "background" level spectral gamma counts were recorded between 10 and $50 \mathrm{ft}$. 


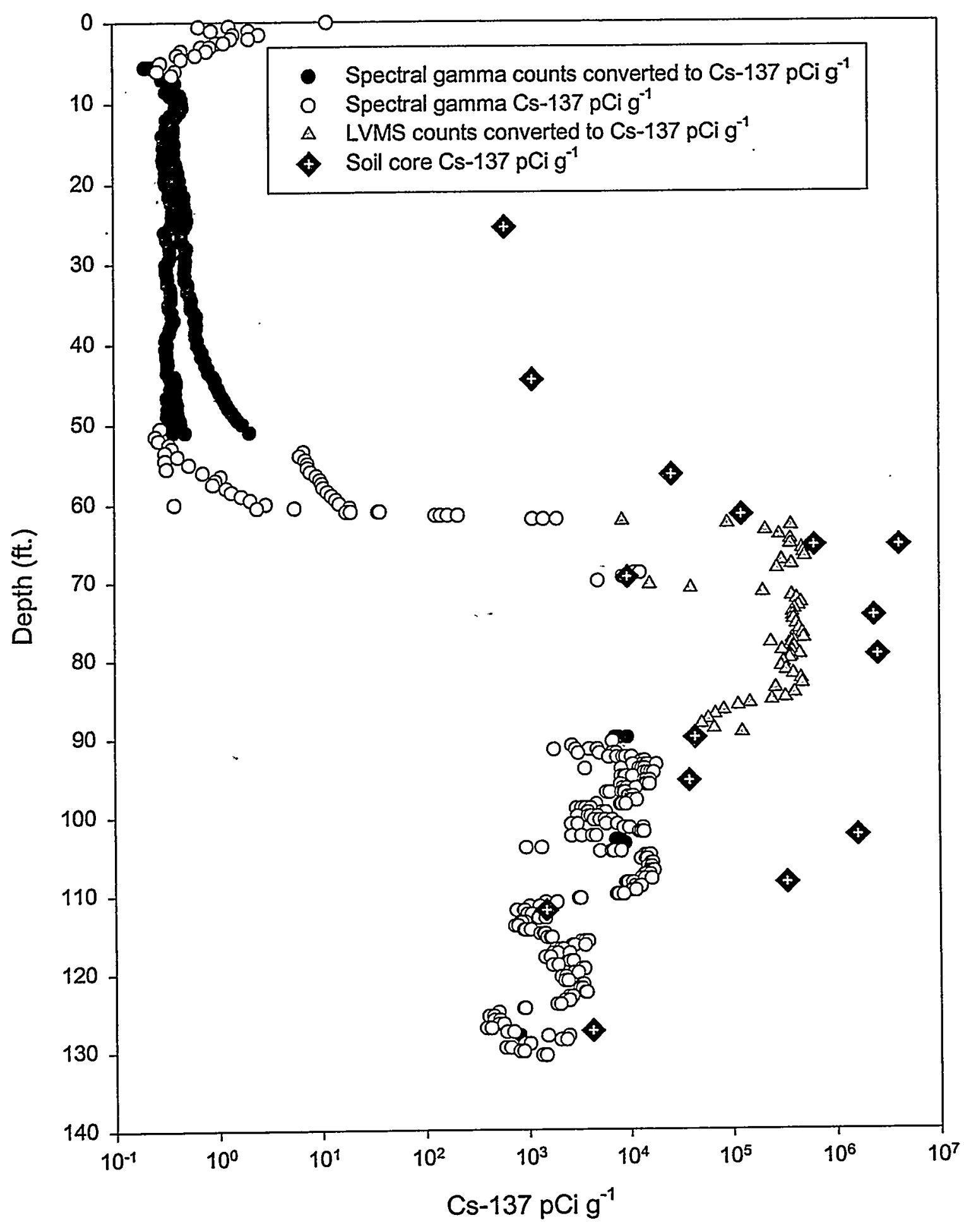

Fig. 21. Drywell 09-39 Cs-137 $\mathrm{pCi} \mathrm{g}^{-1}$ from gamma logging and soil cores. 


\section{References}

Brodeur, J. R. 1996. Unpublished letter report, Subject: "41-12-01 Borehole log report", Rust Geotech Inc., Richland, Washington.

Jones, T. E., Khaleel, R., Meyers, D. A., Shade, J. W., and M. I. Wood. 1998. A summary and evaluation of Hanford site tank farm subsurface contamination. HNF-2603, Lockheed Martin Hanford, Richland, WA.

Randall, R.R. 1999. Unpublished letter report, Subject: "SX red GM scaling for instrument sensitivity changes", Three Rivers Scientific, Richland, Washington.

Raymond, J. R. and E. G. Shdo. 1966. Characterization of subsurface contamination in the SX tank farm. BNWL-CC-701, Battelle-Northwest, Richland, Washington.

U.S. Department of Energy (DOE). 1997. Vadose zone characterization project at the Hanford tank farms, assessment of log data for borehole 41-09-39 and correlation with borehole 41-09-04 in the SX tank farm, GJO-HAN-9, prepared by MACTEC-ERS for the Grand Junction Projects Office, Grand Junction, Colorado.

U.S. Department of Energy (DOE). 1996. Vadose zone characterization project at the Hanford tank farms, SX tank farm report, GJPO-HAN-4, prepared by Rust Geotech for the Grand Junction Projects Office, Grand Junction, Colorado.

Westinghouse Hanford Company (WHC). 1992a. Tank 241-SX-108 leak assessment, WHC-MR300, Westinghouse Hanford Company, Richland, Washington.

Westinghouse Hanford Company (WHC). 1992b. Tank 241-SX-109 leak assessment, WHCMR-301, Westinghouse Hanford Company, Richland, Washington. 


\section{Addenda: ASCII data files}

Six ASCII text files are included with this report on diskette. The $S X$ counts_raw.txt, $S X \_c o u n t s \_$processed.txt, and $S X \_$counts_binned.txt files correspond to the composite gamma counts data set. The $S X \_c u r i e s \_r a w . t x t, \bar{S} X$ _curies_processed.txt, and $S X_{-}$curies_binned.txt files correspond to the composite Cs-137 data set.

\section{The composite gamma counts data set}

$S X$ counts_raw.txt

This file contains the vertical drywell counts, lateral drywell counts, and soil core Cs-137 concentrations used to create the composite gamma counts data set. All values in this file are included as they were found in either the spectral gamma dataset compiled by Mactec-ERS, the historical gamma logging record, or after digitizing Plates I-IX in the Raymond and Shdo (1966) report. The five fields are:

Field 1: a four-digit identification code unique to each vertical drywell, lateral drywell, or soil core. The first two digits identify the SX tank, 01 through 15, associated with each drywell or core. Vertical drywells not associated with a tank are designated with two leading zeroes, i.e. 00. The next two digits of the four-digit code designate individual wells or cores. Vertical drywells are labeled 01 through 39, lateral drywells are labeled 41 through 43, and soil cores are labeled 50 through 59. All identification codes in this field are identical to labels used in Fig. 1.

Field 2: the depth (ft.) from the surface in the case of vertical drywells and soil cores or the distance (ft.) from the surface via caissons and individual laterals.

Field 3: historical or spectral gamma cps in vertical and lateral drywells or concentration of $\mathrm{Cs}-137(\mathrm{pCi} / \mathrm{g})$ in soil cores.

Field 4: the probe type used to acquire data. Probe types 1,2, 4, and 14 correspond to the historical gamma logging instruments. The spectral gamma detector is designated probe type 5 . The LVMS detector is assigned probe type 6. Soil core values are designated type 7.

Field 5: the calendar year individual values were recorded.

\section{SX_counts_processed.txt}

This file contains the complete composite gamma counts data set following conversion of all raw data to consistent units as described in detail in the main body of this appendix. The first two fields are id and depth/distance fields identical to those described above. The third field is gross gamma counts in type $4 \mathrm{cps}$. 


\section{SX_counts_binned.txt}

This file contains the results of binning the composite gamma counts data set. The first three fields are the $x, y$, and $z$ coordinates of the center of mass of the values contained in each non-empty bin in eastings, northings, and depth below surface, respectively (all in meters). The fourth field is the arithmetic mean of the log-transformed values in each bin. The fifth field contains the number of values assigned to each bin. The sixth field is the bin sample variance. If a bin contained only one value, a sample variance was not calculated and an error code of -9.99999 is written.

\section{The composite Cs-137 data set}

SX_curies_raw.txt

This file contains all values used to assemble the composite Cs- 137 data set. The five fields are identical in type to the five fields in the $S X$ counts_raw.txt file. All spectral gamma Cs137 concentrations reported by Mactec-ERS are included and identified as probe type 8 . At locations (well/depth) where spectral gamma total counts were logged but no spectral gamma Cs-137 concentrations were reported, spectral gamma total counts were added to the raw Cs-137 data set. Historical gamma counts from vertical and lateral drywells included in this file are identical to values found in the raw gamma counts data set. Similarly, soil core Cs-137 concentrations are identical to values found in the raw gamma counts data set. At drywell 09-39, LVMS total counts were included over the depth interval where the spectral gamma detector saturated $(62-90 \mathrm{ft}$.$) .$

\section{SX_curies_processed.txt}

This file contains the complete composite Cs-137 data set following processing of the raw data as described in detail in the main body of this appendix. The first two fields are id and depth/distance fields. The third field is $\mathrm{Cs}-137$ concentration in $\mathrm{pCi} \mathrm{g}^{-1}$.

\section{SX_curies_binned.txt}

This file contains the results of binning the composite Cs-137 data set. The six fields are identical in type to those found in the $S X$ _counts_binned.txt file. 


\title{
APPENDIX 2. GEOSTATISTICAL APPROACH USED IN ESTIMATION OF . SX-FARM VADOSE ZONE Cs-137 INVENTORIES *
}

\author{
Daniel Goodman \\ Environmental Statistics Group \\ Biology Department \\ Montana State University \\ Bozeman, MT 59717
}

Januäry 28, 2000

\section{GENERAL FEATURES OF THE MODEL}

\subsection{The Second-Order Stationary Gaussian Process}

The geostatistical model used in this analysis is the basic second-order stationary Gaussian process. It offers a good compromise of features with respect to relative transparency, interpretability, and computational speed in kriging (Goodman, 1999). This model has a long history in the context of time series analysis (Wiener, 1949), and it extends naturally to geostatistics (Cressie, 1991). This model treats the process mean as a distinct parameter, which can be set differently from the actual mean of the data, which may be a biased sample of the process, because of the tendency to sample in hot spots. The separation allows a reasonable "background" prediction value to be established, without detrending. In this sense the model is "purely geostatistical" since no ancillary models of a physical process

* This is a report to MACTEC Inc., under agreement 3751.119.376 with Montana State University. Portions of the work reported here were supported by DOE cooperative agreement DE-FC07-94ID13317 to Montana State University, Daniel Goodman, Principal Investigator. 
are invoked to account for a non-stationary mean. The model for the process is mathematically tractable, allowing a simple form for prediction, called "simple kriging," and for conditional simulation to calculate error distribution of decision quantities. The model can readily deal with observation error, both in prediction and in conditional simulation. The model has a natural likelihood function for the observations, should it be desired to use that approach for parameter estimation.

\subsection{The Exponential Variogram Model}

We chose the exponential variogram model because of its mathematical simplicity, and because it is a popular choice for applications in hydrogeology (Kitanidis, 1997). An exponential decay of correlation with distance seems to accord reasonably well both with intuition of the physical processes, and with the usual data sets.

To minimize the number of parameters, and complexity, we opted for isotropy in the horizontal dimensions. Because the movement of contamination in the vertical dimension in the vadose zone clearly is governed by physical mechanisms that are quite different from those controlling horizontal dispersion, we set a separate parameter for correlation with distance in the vertical dimension.

\subsection{Parameter Estimation}

This model structure requires that 5 parameters be determined. These are:

1. the process mean,

2. the process variance,

3. the correlation decay rate with distance in the horizontal,

4. the correlation decay rate with distance in the vertical,

5. the measurement error variance.

The volume of the tank farm vadose zone was subdivided in a grid system of cells that are cubes of $1 \mathrm{~m}$ on a side. After conversion of the data from various sources to estimated $\mathrm{pCi} / \mathrm{g}$ Cs-137, the log transformed values within each cell were averaged (in the log space, so these are actually geometric means of the concentrations), and the cell mean was employed to represent the contamination in that cell, and this log concentration value was assigned the nominal location of 
the log concentration weighted mean position (in 3-d) of the data locations that went into the mean for that cell. Later, when kriging predictions were made, these were assigned to the respective cell centers in this grid system.

For all cells with two or more data locations contributing to the mean, the within-cell sample variance was used to estimate a corresponding population variance, and the each such variance was graphed against the cell mean. A regression through this scatter plot (Figure 1) showed slight slope, due primarily to the low variances in the frequent cells reporting low contamination. These cells contributed little to the inventory, so their error variance was of little interest. Overall, and also in the subset of cells with higher concentration, the histogram of the variances was very skewed, as would be expected for variances that naturally have a chi-squared distribution. The median variance from this distribution was taken to represent the measurement error variance for a single measurement, and then the measurement error associated with the mean value used for each cell with data was computed as the standard error of that mean, using this estimate of the measurement error variance per measured value. It must be noted that this estimation of measurement error from replicability of measurements was dominated a large number of replications using the same probe type, which resulted in a much lower apparent measurement error than was revealed in comparing measurements, at the same location, with different probe types.

The process mean was set to duplicate the observed "background" in the tank farm as revealed by gross gamma logging with the type 4 instrument which has good resolution at the lower ranges of values, and was used for measurements at a large number of the locations. The gamma logging data with that probe showed a distinct normal mode at $25 \mathrm{cps}$ (Figure 2). The type 2 gross gamma instrument, which could not revolve such low activity levels, showed a second, definitely non-normal mode between $e^{14}$ and $e^{15}$ (shown in a histogram of the log counts in Figure 3). We took the distinct mode observed at $25 \mathrm{cps}$ with the type 4 instrument, which converts to $0.89 \mathrm{pCi} / \mathrm{g}$, to be the local "background," and used this value for the process mean. The very skewed shape of the hotter mode of the counts from the type 2 instrument (as revealed in the histogram of untransformed counts in Figure 4) indicated that some transformation of the data was required. We chose log transformation for the merged data, which resulted in a more normal-looking distribution than the original histogram, but the shape was still far from normal, with a long right-tail to very high, but infrequent values (Figure 5). 


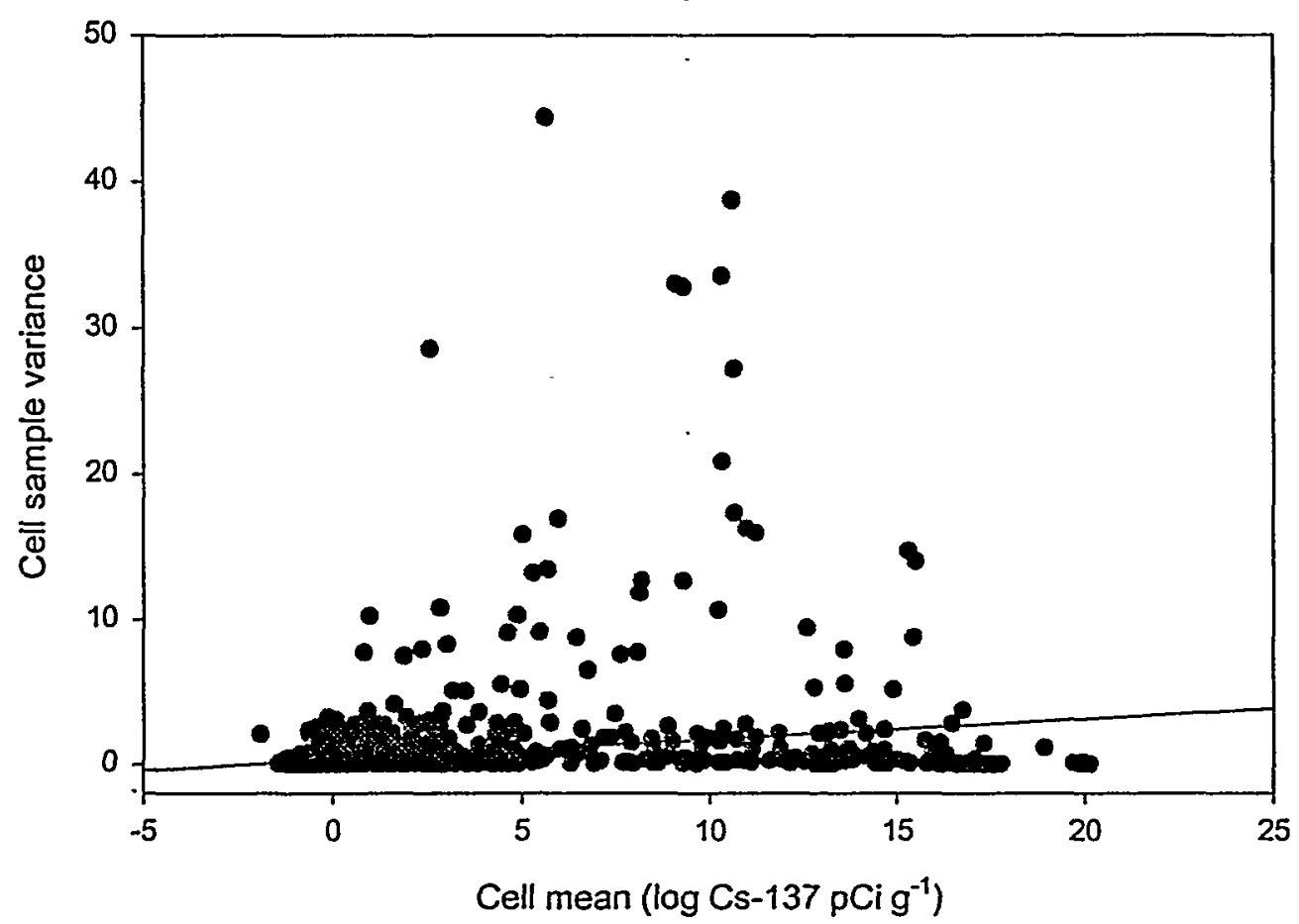

Fig. 1. Cell sample variance vs. cell mean for all cells containing two or more values.

The process variance plays only a minor role in computing the point estimate of the process at a location, as long as it is larger than the measurement error. Under these circumstances it serves only to scale the effect of the measurement error, weighting the data versus the process mean in the point prediction. If measurement error is zero, the process variance has no influence at all on the point prediction. For this same reason, the process variances has the same modest degree of influence on the point estimate of the inventory. However, the process variance plays a very large role in computing the prediction error variance, either for a single location, or for an inventory. The mathematics of the prediction error calculation are more sensitive to the underlying assumptions of the model than are the mathematics of the point prediction calculation. The assumption of normality is crucial to the applicability of the prediction error calculations for purposes of obtaining confidence intervals on the estimates.

The actual distribution of data from SX-farm was far from normal, even after log-transformation, for a long tail of rare but very high values persisted. This 
tail dominated the direct calculation of the variance of the data values, and gave very high values for the variance relative to the mean: the standard deviation was 3.93 in the log space, or $25 \times 10^{6}$ for the untransformed values, operating with data all converted to $\mathrm{pCi} / \mathrm{g}$. Since this very large empirical variance was owing to a severe departure from the assumptions of the model, use of the empirical variance as the process variance would lead to very unreasonable behavior of the prediction error calculation. The dominance of the high "outliers" in establishing such a high estimate of the process variance would result in reasonable estimates of prediction error only for the few data locations with very high concentrations of contamination, and would result in vastly inflated prediction error estimates for most of the rest of the volume.

Since the inventory calculations involve summing over much of the vadose zone volume, it was deemed that the reasonableness of the prediction error variance for the great preponderance of the volume that has relatively low contamination concentrations was more important than the correctness of the prediction error variance for the few high outliers. Accordingly, the process variance was estimated as the variance associated with the central mass of the distribution of the data, excluding the tail of high outliers, giving a value of 1.08 in the log space.

The remaining two parameters were the range of influence parameters (horizontal and vertical) that describe the rate at which correlation decays with increasing distance (in each direction). One conventional way (familiar to physicists) to report this parameter is as the $e$-folding distance: the separation distance at which the correlation between local contamination levels (true values, without measurement error) is $1 / e$ (roughly $37 \%$ ). We employed jack-knife cross-validation, in the kriging model itself, to adjust the two free range-of-influence parameters to minimize the jack-knife residual. Because this operation was carried out within a model that already embodied all the other assumptions we had made, and parameters we had set, the jack-knife parameter estimation had the further advantage of optimizing the two free parameters in context, so that any mis-specifications could be compensated to the extent possible. Similarly, the jack-knife predictions error would be representative of the actual prediction errors, at similar separation distances, in this application.

The parameter estimates from the final merged data set gave an $e$-folding distance of about 8.2 meters in the horizontal and 3.0 in the vertical. The residual response surface from the jack-knife cross validation was steep in the direction of smaller values of these parameters, but had a shallow slope in the direction of larger parameter values (see Figure 6). 


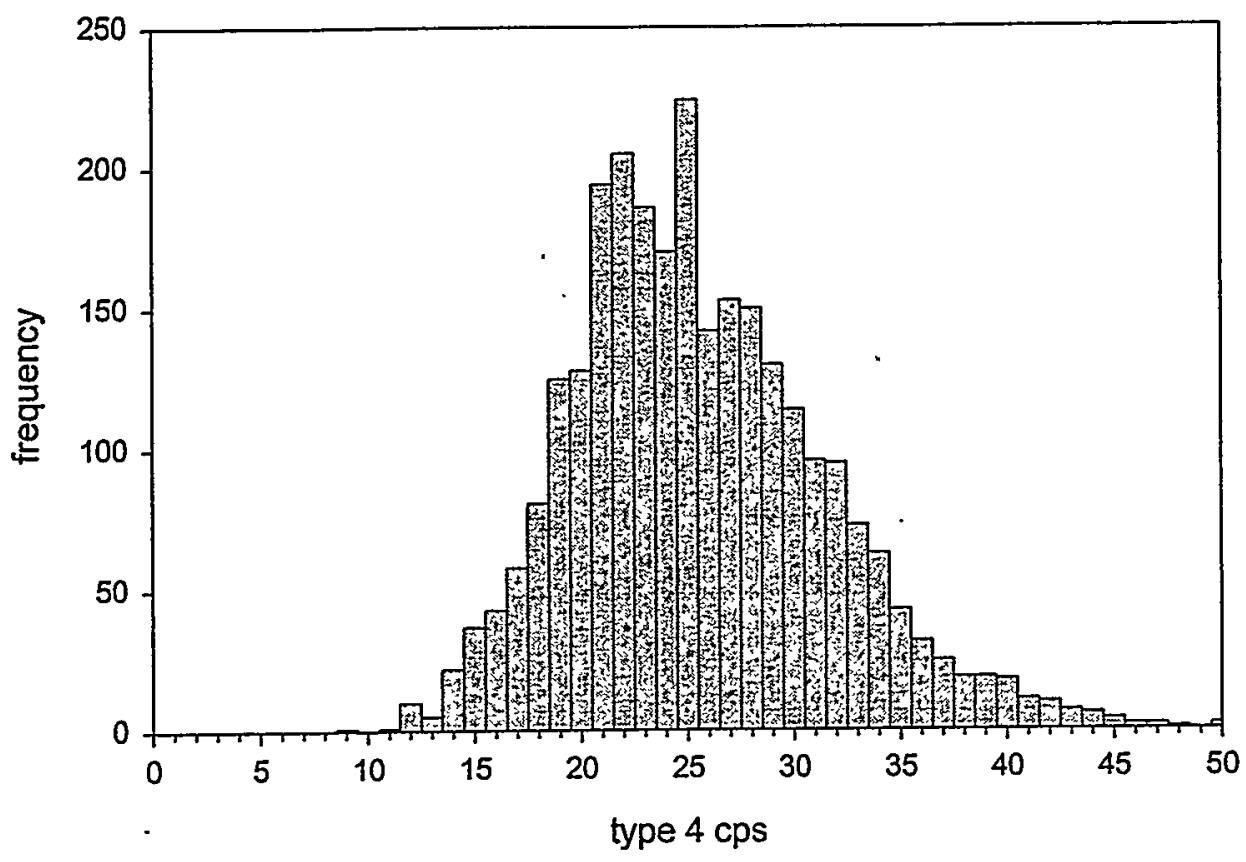

Fig. 2. Histogram of 1994 gamma-logging values less than $50 \mathrm{cps}$ recorded with the type 4 detector in the SX-108, SX-109 six-tank neighborhood.

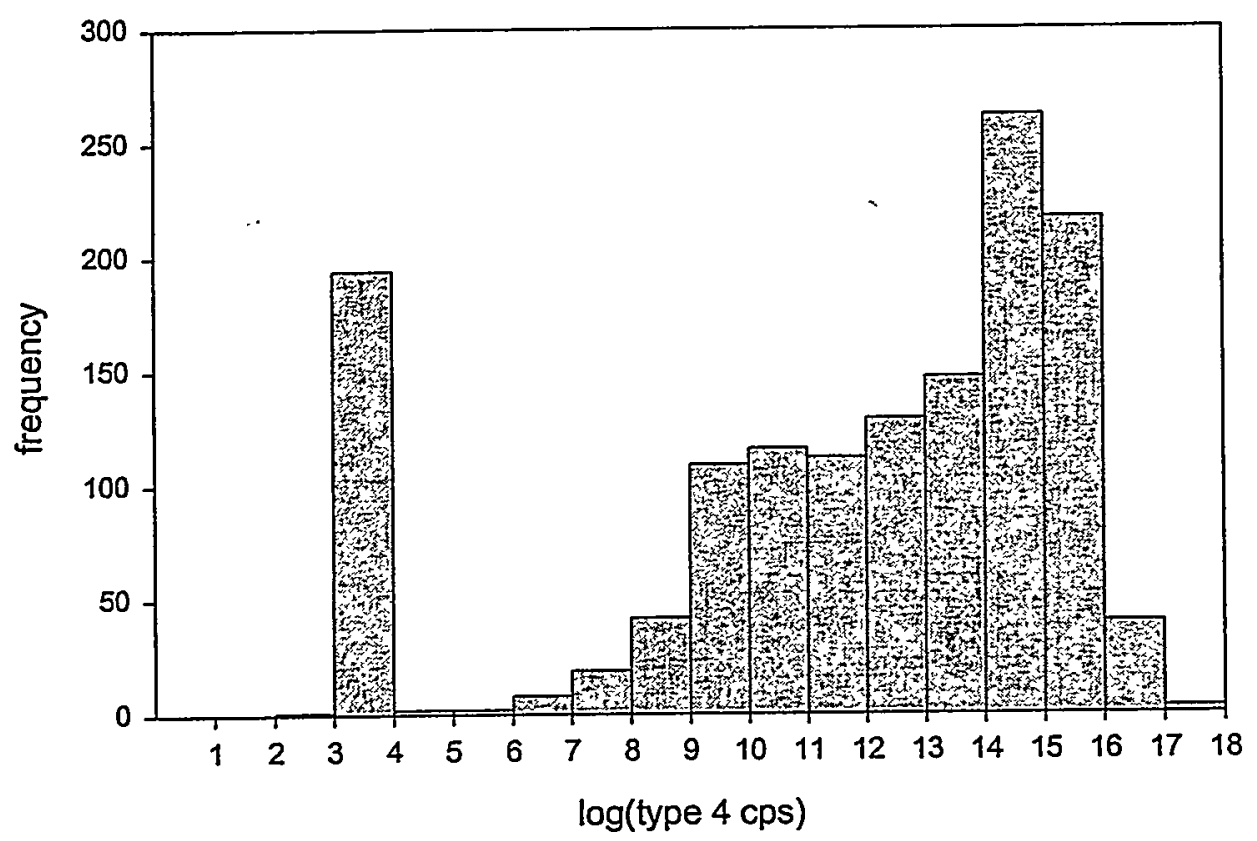

Fig. 3. Histogram of type 2 gamma logging converted to type $4 \mathrm{cps}$. 


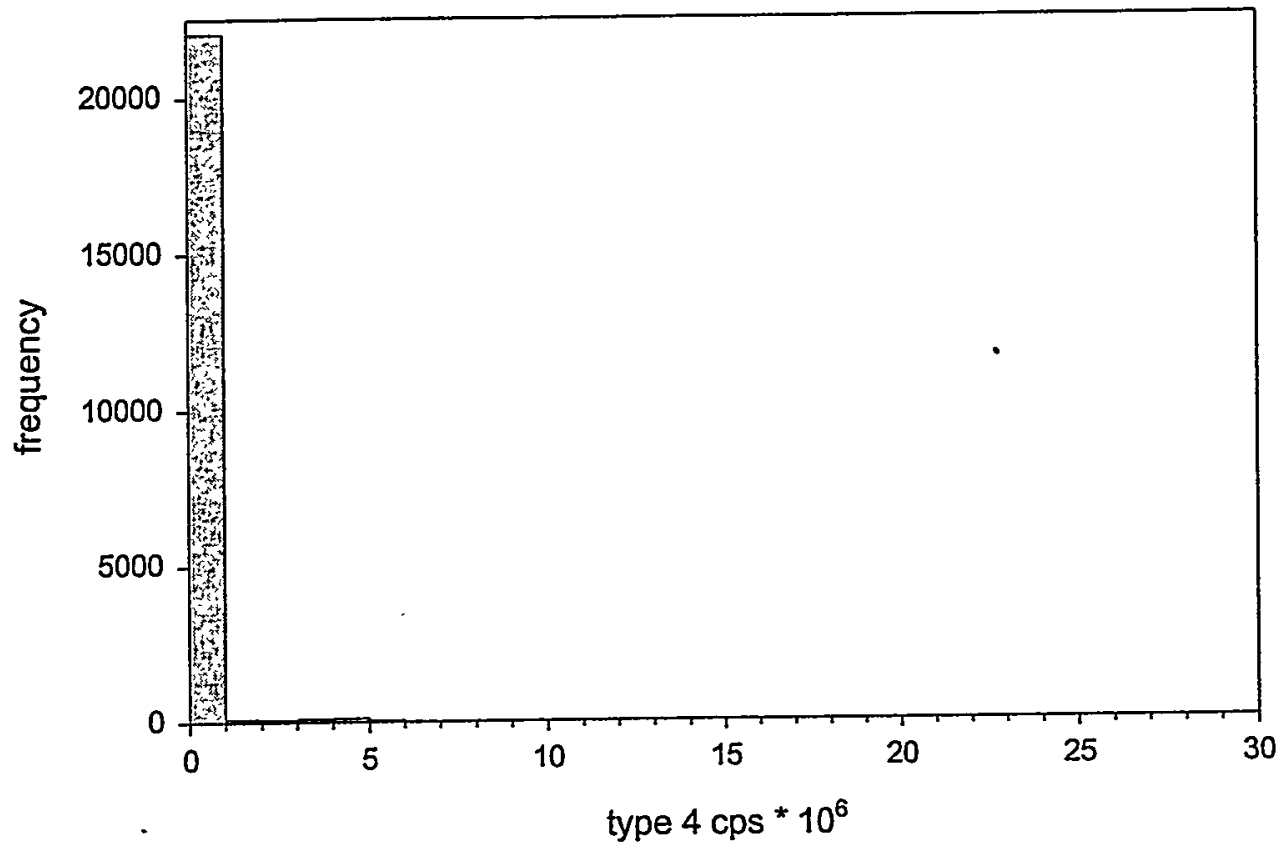

Fig. 4. Histogram of all gross gamma counts in type 4 units.

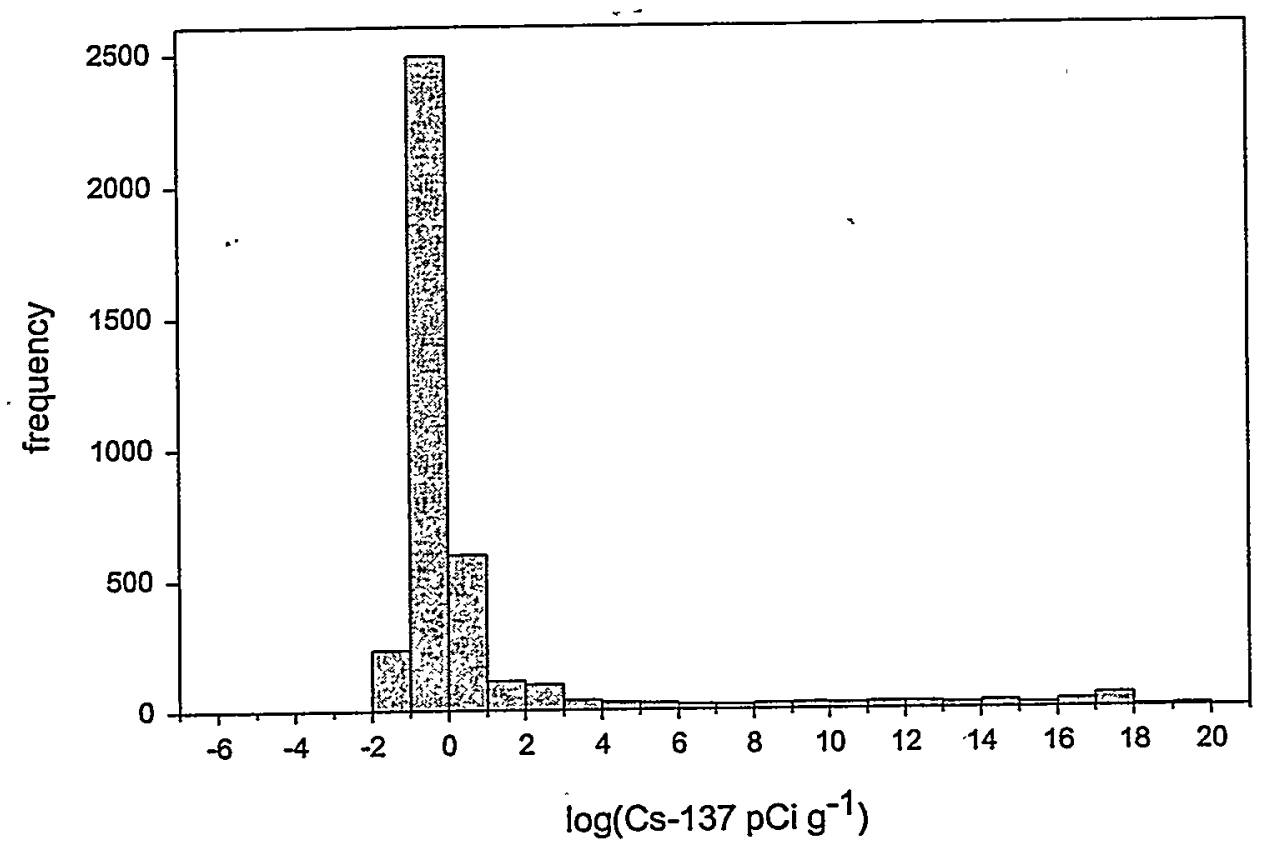

Fig. 5. Histogram of $\log \left(\mathrm{Cs}-137 \mathrm{pCi} \mathrm{g}^{-1}\right)$ in all cells. 


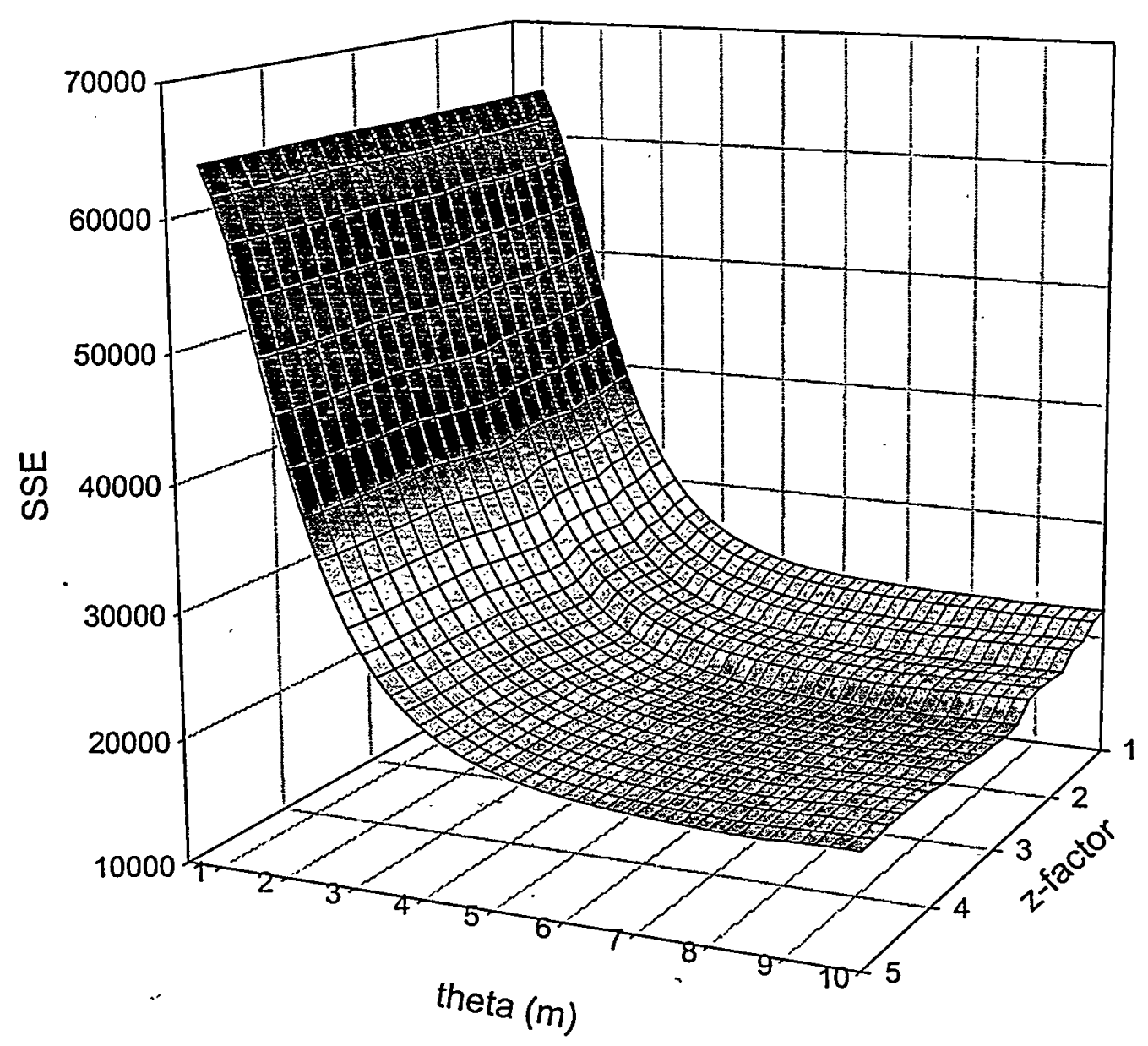

Fig. 6. Jack-knife residual surface resulting from jack-knifing the log-transformed and binned composite Cs-137 data set (SSE minimum at theta $=8.2 \mathrm{~m}, \mathrm{z}=2.7$ ). 


\section{MATHEMATICAL REPRESENTATION}

\subsection{Notation}

$k \quad$ number of dimensions

$n \quad$ number of locations with observations

$m$ number of locations where value of process is to be predicted

y n-element vector of observed values, indexed by location, where $y_{i}$ is observed value at location $i$, and where $i$ is a $k$-element vector of coordinates in a cartesian space

$\underline{x} \quad$ n-element vector of unobserved values, of the underlying process, indexed by location, where $x_{i}$ is value of the process at location $i$, and where $i$ is a $k$-element yector of coordinates - in a cartesian space

$\sigma_{x}^{2} \quad$ variance of underlying process generating values of $x$

$\sigma_{\epsilon}^{2} \quad$ variance of observation error

$\mu_{x} \quad$ mean of underlying process generating values of $x$

$C_{w z} \quad$ covariance matrix of random vectors $w$ and $z$, where $c_{w z}(i, j)$, element $(i, j)$ of this matrix, is the covariance between element $i$ of vector $w$ and element $j$ of vector $z$

$\underline{\alpha} \quad k$-element vector of scale factors to rescale distance from origin along each dimension for purposes of computing a distance measure which weights each dimension separately

$d_{\underline{\alpha}}(i, j) \quad \alpha$-scaled distance between locations $i$ and $j$ computed as a euclidean distance after rescaling the distance from the origin along coordinate $h$ by $\alpha_{h}$

\subsection{Model of the Underlying Process}

$$
\begin{aligned}
& \underline{x} \sim N\left(\underline{\mu_{x}}, C_{x x}\right) \\
& x_{i} \sim N\left(\mu_{x}, \sigma_{x}^{2}\right)
\end{aligned}
$$

2.3. Spatial Structure of the Underlying Process

$$
\begin{gathered}
c_{x x}(i, j)=f\left(d_{\underline{\alpha}}(i, j)\right) \\
f\left(d_{\underline{\alpha}}(i, j)\right)=\sigma_{x}^{2} e^{-d_{\underline{\alpha}}(i, j)}
\end{gathered}
$$




$$
\begin{gathered}
c_{x x}(i, i)=\sigma_{x}^{2} \\
d_{\underline{\alpha}}(i, j)=\left(\sum_{h=1}^{n}\left(\frac{i_{h}}{\alpha_{h}}\right)^{2}\right)^{\frac{1}{2}}
\end{gathered}
$$

2.4. Relating Observations to the Underlying Process

$$
\begin{gathered}
y_{i}=x_{i}+\epsilon_{i} \\
\epsilon_{i} \sim N\left(0, \sigma_{\epsilon}^{2}\right)
\end{gathered}
$$

2.5. Spatial Structure of the Observations

$$
\begin{aligned}
& c_{y y}(i, j)=c_{x x}(i, j) \\
& c_{y y}(i, i)=\sigma_{x}^{2}+\sigma_{\epsilon}^{2}
\end{aligned}
$$

2.6. Joint Spatial Structure of Observations and the Underlying Process

$$
\begin{gathered}
c_{x y}(i, j)=c_{x x}(i, j) \\
c_{x y}(i, i)=\sigma_{x}^{2}
\end{gathered}
$$

2.7. Joint Distribution of Observations and the Underlying Process

$$
\begin{gathered}
\underline{v}=\left(\frac{\underline{y}}{\underline{x}}\right) \\
C_{v v}=\left(\begin{array}{ll}
C_{y y} & C_{y x} \\
C_{x y} & C_{x x}
\end{array}\right)
\end{gathered}
$$


2.8. Conditional Distribution of the Process, Given the Observations

$$
\begin{gathered}
(\underline{x} \mid \underline{y}) \sim N\left(\underline{\mu}_{(\underline{x} \mid \underline{y})}, C_{(\underline{x} \mid \underline{y})(\underline{x} \mid \underline{y})}\right) \\
\underline{\mu_{(\underline{x} \mid \underline{y})}}=\underline{\mu_{x}}+C_{x y} C_{y y}^{-1}\left(\underline{y}-\underline{\mu_{x}}\right) \\
\left.C_{(\underline{x} \mid \underline{y})(\underline{x} \mid \underline{y})}\right)=C_{x x}-C_{x y} C_{y y}^{-1} C_{y x}
\end{gathered}
$$

2.9. Point Estimate of the Process at a Location

$$
\widehat{x}_{i}=\mu_{x}+C_{x_{i} \underline{y}} C_{\underline{y y}}^{-1}\left(\underline{y}-\underline{\mu_{x}}\right)
$$

2.10. Prediction Error Variance of Point Estimate at a Location

$$
\begin{aligned}
\sigma_{\widehat{x}_{i}}^{2} & =E\left(\widehat{x}_{i}-x_{i}\right)^{2} \\
& =\sigma_{x}^{2}-C_{x_{i} \underline{y}} C_{\underline{y y}}^{-1} C_{\underline{y} x_{i}}
\end{aligned}
$$

2.11. Jack-knife Residual Variance at a Location

$$
E\left(\widehat{x}_{i}-y_{i}\right)^{2}=E\left(\widehat{x}_{i}-x_{i}\right)^{2}+\sigma_{\epsilon}^{2}
$$

2.12. Point Estimate of Inventory over $m$ Locations

$$
E\left(\sum_{i=1}^{m} x_{i}\right)=\sum_{i=1}^{m} \widehat{x}_{i}
$$

2.13. Prediction Error Variance of Inventory over $m$ Locations

$$
E\left(\sum_{i=1}^{m} \widehat{x}_{i}-\sum_{i=1}^{m} x_{i}\right)^{2}=\sum_{i=1}^{m} \sum_{j=1}^{m} c_{(\underline{x} \mid \underline{y})(\underline{x} \mid \underline{y}) i j}
$$

\subsection{Likelihood Function}

$$
\ln \left(L\left(\mu_{x}, \sigma_{x}, \underline{\alpha}\right)\right)=-\frac{n}{2} \ln (2 \pi)-\frac{1}{2} \ln \left(\left|C_{x x}\right|\right)-\frac{1}{2}\left(\underline{x}-\underline{\mu_{x}}\right)^{T} C_{x x}^{-1}\left(\underline{x}-\underline{\mu_{x}}\right)
$$




\subsection{Computational Demands and Computational Strategy}

For a data set with observations at $n$ locations, the kriging equation requires inversion of an $n \times n$ matrix, and this exacts a computational cost proportional to $n^{3}$. The inversion, however, only needs to be done once for obtaining the full set of $m$ point predictions for a given data set, once the process variance and spatial correlation parameter values are set.

A point estimate of the (predicted) process value at a given location requires, at each new location to be interpolated, an application of the $n \times n$ inverse matrix in multiplication to an $n$-element vector to obtain the vector of kriging weights, $w_{i}$, for the data relative to that location, and then a dot product of two $n$-element vectors to apply those weights to the data in order to obtain the prediction. The vector of kriging weights,

$$
\underline{w_{i}}=C_{x_{i} \underline{\underline{y}}} C_{\underline{y y}}^{-1} \quad,
$$

again appears as an intermediate product in later calculations of prediction error variance, so an efficient computational scheme will use each set of kriging weights for all needed purposes, when first computed, rather than recompute them.

If we consider the basic computational unit in these various matrix operations to be the combination of a floating point multiplication and a floating point addition, then we see that the point estimate for each location to be predicted requires $n^{2}+n$ such units of floating point operation, plus two more floating point additions to remove and then restore the process mean. So, overall, a simple kriging prediction, without error analysis, for a field of $m$ points to be predicted, exacts a computational cost of $m \times n^{2}+m \times n$ basic units of floating operation, plus $2 \times m$ more floating point additions. In other words, the computational cost is approximately proportional to $m \times n^{2}$.

Embedding the kriging prediction in a jack-knife cross-validation for parameter estimation, further multiplies the computational cost by the number of combinations of parameter values explored, and multiplies the cost of the inversion of $n \times n$ matrix, since a new such matrix must be inverted for each new combination of parameters defining the process mean and the spatial correlation.

Embedding the kriging prediction in an inventory calculation does not involve appreciable additional expense for the point estimate of the inventory, since that is simply a sum of the point estimates for each cell in the volume.

A calculation of the prediction error variance for the point estimate of the (predicted) process value at a given location requires, at each new location to 
be interpolated, an application of the $n \times n$ inverse matrix in multiplication to an $n$-element vector to obtain the kriging weights for the data relative to that location, and then a dot product of two $n$-element vectors in order to obtain a quantity rather like a regression " $r$ squared," which finally is subtracted from the process variance to obtain the prediction error variance. Thus the prediction error variance at each location to be predicted requires $n^{2}+n$ basic units of floating point operation, plus one more floating point addition. Efficient programming can reduce the additional cost of the prediction error calculation over the cost of the prediction of the process at a given location, since the first $n^{2}$ operations duplicate the calculation of the kriging weights from the calculation of the point estimate of the process at that location.

A calculation of the prediction error variance for an inventory is much more expensive than the sum of the prediction errors for the cells in the volume. A substantial portion of the prediction error for a volume is owing to the correlation in prediction error across cells. Therefore the prediction error covariance matrix for all the cells in the volume must be computed. For a volume large enough that the number of cells it comprises is large, this becomes a significant cost. The prediction error covariance matrix, for a volume of $m$ cells, requires application of an $m \times n$ matrix in multiplication to the $n \times n$ inverse matrix, and the resulting $m \times n$ matrix is applied in multiplication to an $n \times n$ matrix to obtain an $m \times m$ matrix. This consumes $m^{3}+n^{2}+m$ basic units of floating point operation. The final substraction to obtain the $m \times m$ covariance matrix of the prediction error for all the cells in the volume adds $m^{2}$ more floating point additions, and summing the elements of the prediction error covariance matrix to obtain the variance for the inventory adds another $m^{2}$ floating point additions. In other words, if the number of cells substantially exceeds the number of data locations, the cost of computing the prediction error for the inventory is approximately proportional to $m^{3}$.

A considerable computation savings can be achieved in computing the prediction error for the inventory by recognizing that the first intermediate matrix multiplication $C_{\underline{x y}} C_{\underline{x} \underline{y}}^{-1}$ creates an $m \times m$ matrix, each row of which is simply the $m$-element vector of kriging weights for the prediction location corresponding to that index. Thus, all the elements of this $m \times n$ matrix, that exacts a computational cost of $m \times n^{2}$ units of floating point operation, will have already been calculated during the course of calculating the point estimates of the predicted value for each cell in the inventory volume (and the dot product of each kriging weight vector with the columns of $C_{\underline{y} \underline{\underline{x}}}$ can be cumulated on the fly). 
Conditional simulation of a field of $m$ points, conditioned on a data set of $n$ points, requires the initial cost of computing a point estimate for each location in the field and a prediction error covariance matrix for all the locations in the field (essentially equivalent to the cost of the point estimate and prediction error variance for an inventory of that field), followed by inversion of the $m \times m$ covariance matrix for the prediction error for all the locations in the field. Then, each realization of the field requires generation of $m$ normal variates and application of an $m \times m$ composition matrix in multiplication to the $m$-element vector. Generally, several thousand realizations will be required for satisfactory characterization of the distribution of a decision quantity. If $\ell$ is the number of realizations, conditional simulation exacts a computational cost proportional to $\ell \times m^{2}$ plus the initial cost proportional approximately to $m^{3}$ and the inversion of an $m \times m$ matrix. .

Two bottlenecks, therefore, can limit the feasibility of the application. One is the size of the matrices that must be inverted; the second is the brute number of basic floating point unit operations. The Cholesky decomposition offers efficiency and stability for the inversion of these positive-definite real symmetric matrices (Ripley, 1981; Ng \& Peyton, 1993). The brute number of multiplications can be controlled by a strategy of limiting the number of prediction points $m$ to the minimum needed to really answer the question (rather than just densely filling the field to get an attractive "picture"), and limiting the number of predictor points used for each prediction to a neighborhood of a small multiple of the $e$-folding distance around the location to be predicted (since more distant data will have little influence).

For the prediction error variance of an inventory, the brute number of floating point calculations can be excessive. If the volume is defined by a $3-d$ grid of 10 cells to a side, $m=10^{3}$ so the computational cost of the inventory is $m^{3}=10^{9}$ floating point unit operations. For a volume defined by a 3-d grid of 20 cells to a side, the computational cost escalates to $512 \times 10^{9}$ floating point unit operations. On a current (1999) PC, a million floating point unit operations take from about 0.1 to 0.5 seconds. Therefore the prediction error variance for an inventory for the 3-d grid of 10 cells to a side will take a matter of minutes, but the 3-d grid of 20 cells to a side will take a matter of days. A good workstation is about 20 times faster than a PC, reducing the time to a matter of hours for calculating the prediction error variance of the inventory for a 3-d grid of 20 cells to a side. Calculation of the prediction error variance of the inventory for a 3-d grid of much more than 20 cells to a side, in a reasonable amount of time, will require a class 
of computer substantially faster than a workstation.

\section{ILLUSTRATION FOR A SINGLE DATA LOCATION}

\subsection{Decay of Data Influence with Distance}

Consider a data set with data at a single location $i$. Then the point prediction for location $j$ at rescaled distance $d_{i} j$ is, following equation [8],

$$
\begin{aligned}
\widehat{x_{j}} & =\mu_{x}+\left(\sigma_{x}^{2} e^{-d_{i j}}\right)\left(\sigma_{x}^{2}+\sigma_{\epsilon}^{2}\right)^{-1}\left(y_{i}-\mu_{x}\right) \\
& =y_{i} \frac{\sigma_{x}^{2} e^{-d_{i j}}}{\sigma_{x}^{2}+\sigma_{\epsilon}^{2}}+\mu_{x}\left(1-\frac{\sigma_{x}^{2} e^{-d_{i j}}}{\sigma_{x}^{2}+\sigma_{\epsilon}^{2}}\right)
\end{aligned}
$$

which shows how the prediction is a weighted average of the process mean and the data value, where the weighting of the data value declines with separation distance and observation error. The weight of the data value goes to zero at large separation distance, and the maximum weight for the data value, $\frac{\sigma_{x}^{2}}{\sigma_{x}^{2}+\sigma_{\varepsilon}^{2}}$, is achieved at zero separation distance.

If the observation error variance is zero, the point prediction simplifies to

$$
\widehat{x_{j}}=y_{i} e^{-d_{i j}}+\mu_{x}\left(1-e^{-d_{i j}}\right) \quad .
$$

With zero observation error variance, the weighting for the data value will be $1 / 2$, for example, at a separation distance of

$$
\begin{aligned}
d_{i j} & =-\ln \frac{1}{2} \\
& =0.6931
\end{aligned}
$$

The prediction error variance for the point prediction at location $j$ is, following equation [19],

$$
\begin{aligned}
\sigma_{\widetilde{x}_{j}}^{2} & =\sigma_{x}^{2}-\left(\sigma_{x}^{2} e^{-d_{i j}}\right)\left(\sigma_{x}^{2}+\sigma_{\epsilon}^{2}\right)^{-1}\left(\sigma_{x}^{2} e^{-d_{i j}}\right) \\
& =\sigma_{x}^{2}\left(1-\frac{\sigma_{x}^{2} e^{-2 d_{i j}}}{\sigma_{x}^{2}+\sigma_{\epsilon}^{2}}\right)
\end{aligned}
$$


which shows how the prediction error variance increases with process variance, error variance, and separation distance, from a minimum of $\frac{\sigma_{x}^{2} \sigma_{\varepsilon}^{2}}{\sigma_{x}^{2}+\sigma_{\epsilon}^{2}}$ at zero separation distance, to a maximum approaching the process variance at large separation distance.

If the observation error variance is zero, the prediction error variance simplifies to

$$
\sigma_{\bar{x}_{j}}^{2}=\sigma_{x}^{2}\left(1-e^{-2 d_{i j}}\right)
$$

With zero observation error variance the prediction error variance will be half the process variance, for example, at a separation distance of

$$
\begin{aligned}
d_{i j} & =-\frac{1}{2} \ln \frac{1}{2} \\
& =0.3466
\end{aligned}
$$

\section{BACK-TRANSFORMING FROM THE LOG SPACE FOR INVENTORY CALCULATIONS}

In our kriging of the Cs-137 data from SX farm, we used log values (logarithm of $\mathrm{pCi} / \mathrm{g}$ ) to characterize the contamination at each location, because we found that the histogram of log values more nearly conformed to the assumption of a normal (Gaussian) process than did the values in original units (counts or $\mathrm{pCi} / \mathrm{g}$ ).

This commits us to assuming that the assumptions of the kriging model apply to this space for these data. I.e., we must assume that the set of values made up of logs of the $\mathrm{pCi} / \mathrm{g}$ values are a realization of a second order stationary Gaussian process characterized by a constant process mean and a constant process variance and a covariance structure determined entirely by separation distance, all in the log space.

Therefore the predicted values at each location, emerging from the kriging calculation are also in logarithms of $\mathrm{pCi} / \mathrm{g}$. Since there is a substantial prediction error associated with each such point prediction, the back-transformation to express the results in $\mathrm{pCi} / \mathrm{g}$ should treat this as back-transformation of a distribution rather than simple back-transformation of a value.

We chose to assume, as per the assumptions of the kriging model, that the distribution of the prediction error in the space of the kriging operation (which in this case was the log space) is normal, so that the point prediction and the 
prediction error variance actually reflect properties of the respective lognormal distribution of values in the space of $\mathrm{pCi} / \mathrm{g}$. Therefore, we take $\widehat{x_{i}}$ the point prediction delivered by the kriging operation for the process value at a given location to be the mean of the normal distribution of values obtained by log transforming the contamination value as measured in $\mathrm{pCi} / \mathrm{g}$, and we take $\sigma_{\hat{x}_{\hat{i}}}^{2}$, the prediction error variance for that point prediction, to be the variance of the normal distribution of values obtained by $\log$ transforming the contamination value as measured in $\mathrm{pCi} / \mathrm{g}$.

Let $\widehat{x_{i}^{*}}$ designate the mean of the lognormal distribution of the values in $\mathrm{pCi} / \mathrm{g}$. Then

$$
\widehat{x_{i}^{*}}=e^{\widehat{x_{i}}+\sigma_{\bar{x}_{i}}^{2} / 2}
$$

and we use this as the point prediction, at this location, in units of $\mathrm{pCi} / \mathrm{g}$.

Under the same assumptions, the prediction error variance for the value at a given location, in $\mathrm{pCi} / \mathrm{g}$, is expressed as the variance of the lognormal distribution of the values in $\mathrm{pCi} / \mathrm{g}$. Let $\sigma_{\widehat{x}_{i}^{*}}^{2}$ designate variance of the lognormal distribution of the values in $\mathrm{pCi} / \mathrm{g}$. Then

$$
\sigma_{\widehat{x_{i}^{*}}}^{2}=e^{2 \widehat{x_{i}}} e^{\sigma \frac{2}{x_{i}}}\left(e^{\sigma \frac{2}{x_{i}}}-1\right)
$$

We note, of course, that this prediction error, in the space of $\mathrm{pCi} / \mathrm{g}$, now is assumed to be a non-standard lognormal distribution, so we would not compute a $95 \%$ confidence interval simply as the point estimate plus or minus twice the square root of the variance.

The inventory, in $\mathrm{pCi} / \mathrm{g}$, is a sum of point predictions in $\mathrm{pCi} / \mathrm{g}$. The point estimate for the inventory, in $\mathrm{pCi} / \mathrm{g}$, therefore is computed as the sum of the back-transformed point estimates for each cell in the inventory volume.

The prediction error variance for an inventory in $\mathrm{pCi} / \mathrm{g}$ is more complicated, and not approximated as satisfactorily. We ignore the effect of the transformation on the correlations between prediction error at different locations, assuming that the correlation is the same in the two spaces, and merely correct for the effect of transformation of the respective variances that normalize the covariance to a correlation. Letting $c_{i j}^{*}$ designate our approximation to the prediction error covariance in the space of the $\mathrm{pCi} / \mathrm{g}$ values, we thus replace each element $c_{i j}$ of the prediction error covariance matrix delivered by the standard calculation (carried out in the log space using equation [17]) according to 


$$
\begin{aligned}
c_{i j}^{*} & =c_{i j} \sqrt{\frac{c_{i i}^{*} c^{*} j j}{c_{i i} c_{j j}}} \\
& =c_{i j} \sqrt{\frac{\sigma_{\widehat{x_{i}^{*}}}^{2} \sigma_{\widehat{x_{j}^{*}}}^{2}}{\sigma_{\widehat{x}_{i}}^{2} \sigma_{\widehat{x_{j}}}^{2}}} .
\end{aligned}
$$

Then the prediction error variance for the inventory, in $\mathrm{pCi} / \mathrm{g}$, is computed as the sum of these approximate back-transformed elements of the prediction error covariance matrix. In this space, the prediction error variance of the inventory, will not be exactly normal, though it will be more normal than a lognormal, since it is a sum of many lognormals that are only partially correlated, and so some of the behavior underlying the central limit theorem will apply.

The approach, outlined in this section, of back-transforming the inventory calculation from logarithms to $\mathrm{pCi} / \mathrm{g}$ by trying to reconstruct the mean and variance of a lognormal distribution from the mean and variance of the normal distribution of the log values, uses formulas which assume that the distribution really is lognormal. We of course have no assurance that this is exactly true.

\section{EFFECTS OF THE BACK-TRANSFORMATION: ILLUSTRATION WITH ONE DATA LOCATION}

\subsection{Increase of Lognormal Mean of the Predictive Distribution with Distance}

If the process is assumed normal in the log space, then the normal prediction error in this space is actually a log-normal distribution in the space of the untransformed units. Then the point prediction in the space of the untransformed units should be computed as the expectation (mean) of the lognormal predictive distribution, according to equation [31]. In the worst case of separation distance so large that the prediction in the log space takes the limiting value of the process mean with the prediction error variance taking the limiting value of the process variance, then the point prediction in the space of the untransformed units is $e^{\mu_{x}+\sigma_{x}^{2}}$.

In the present application where $\mu$ was chosen to represent "background" in $\log$ units, $e^{\mu}$ recapitulates "background" in the original units, and so the backtransformation gives a point prediction that is $e^{\sigma_{x}^{2}}$ times "background." Is this reasonable? The answer depends upon what we mean by "background." 
If by "background" we mean the values within the area of the SX farm that could plausibly be contaminated, but where we don't have data, then a point prediction which responds to the possibility of contamination is not unreasonable. In the present application, where the process mean in the log space is -0.111 (corresponding to a "background" value of $0.89 \mathrm{pCi} / \mathrm{g}$ ) and the untruncated process variance in the log space is 15.5 , then the back-transformation for a point prediction at a location far from any data gives a mean for the log-normal of 2,322 $\mathrm{pCi} / \mathrm{g}$, which is 2,078 times the measured background. This is not unreasonable when the upper $95 \%$ tail on the process distribution in the log space is 7.76 . corresponding to $2,352 \mathrm{pCi} / \mathrm{g}$, and the largest measured values for contamination are in excess of $100 \times 10^{6} \mathrm{pCi} / \mathrm{g}$.

If, on the other hand, we mean by "background" the ground state at locations that are not contaminated, then of course the back-transformation will be viewed as creating a bias that raises this "background." The trouble, though, is justifying, in the absence of nearby measurements, that a given location really is in such an area of low contamination.

For a gauge of how close to a data location the prediction location must be in order for the back-transformation to raise the point prediction by an appreciably smaller multiple than the worst case, consider the illustration of a prediction at location $i$ from a single data location $j$. If the separation distance is 0.3466 , then in the log space the prediction error variance is half the process variance in the log space, so the multiple applied in the back-transformation is $e^{\sigma_{x}^{2} / 4}$, i.e., the square root of the multiple for the worst case. For the untruncated process variance in the present application, the multiple over this short distance is 48 .

\section{EFFECTS OF THE ASSUMPTIONS ON THE INVENTORY ESTIMATES}

\subsection{Point Estimate of the Inventory}

We see from equation [21] that the point estimate of the inventory is simply the sum of the point estimates of the cells in the selected volume. The kriging point estimate is a robust quantity, relatively insensitive to departures from assumptions of the model. For example, a process with local differences in the process mean (this is called a "trend" in the geostatistical literature) will still be fit reasonably well by the point predictions, if there is reasonable data density, since the point predictions track the data, and the data will track the local dif- 
ferences in the process mean. Also, our procedure for determining the $e$-folding distance parameters by jack-knife cross-validation automatically compensates to some degree for possible mistakes in specifying the other parameters, since it adjusts the $e$-folding distance in context for best fit to the data. And finally, when measurement error variance is small relative to the process variance, neither has appreciable influence on the point predictions. Thus we have strong reasons for expecting the point estimates of the inventory to behave reasonably, and to give good, and stable, values.

In the actual event, the inventory estimates for SX-Farm lived up to this expectation. Two features of the inventory estimates may strike some readers as anomalous, but even in these two respects we believe the estimates are correct within the limitations of the data. One apparent anomaly is the low inventory estimate for the plume under SX-115, relative either to the calculated leak loss inventory from the tank, or the vadose zone inventory estimate calculated by Raymond and Shdo (1966). The Raymond and Shdo estimate was calculated from much the same data that we used, but with a very different procedure for converting the gamma count data from the laterals to $\mathrm{pCi} / \mathrm{g}$ : they simply equated the highest counts in laterals to the highest $\mathrm{pCi} / \mathrm{g}$ measurements from their soil core samples around the perimeter of the tanks, some distance away from the hottest portions of the laterals. The Raymond and Shdo procedure is not unreasonable, but it gives a much different conversion factor than we obtained from comparing spectral gamma total count data and the spectral gamma Cs-137 values for the same location and from comparing the Cs- 137 measurements reported by Raymond and Shdo for the cores around tank SX-108 and our kriged point predictions of the gamma counts in those locations based on the gamma counts measured elsewhere (Figure 7). The comparison of soil core sample measurements from drywell 09-39 with the co-located gamma logging data (Figure 8) suggests a higher conversion from counts to $\mathrm{pCi} / \mathrm{g}$, but this is still not high enough to obtain an inventory estimate for the SX-115 vadose zone that would be similar to the Raymond and Shdo estimate for that inventory. 




Fig. 7. Spectral gamma Cs-137 $\mathrm{pCi} \mathrm{g}^{-1}$ vs. spectral gamma total counts in type 4 units and SX-108 soil core Cs-137 $\mathrm{pCi}^{-1}$ vs. kriging-predicted type $4 \mathrm{cps}$. 


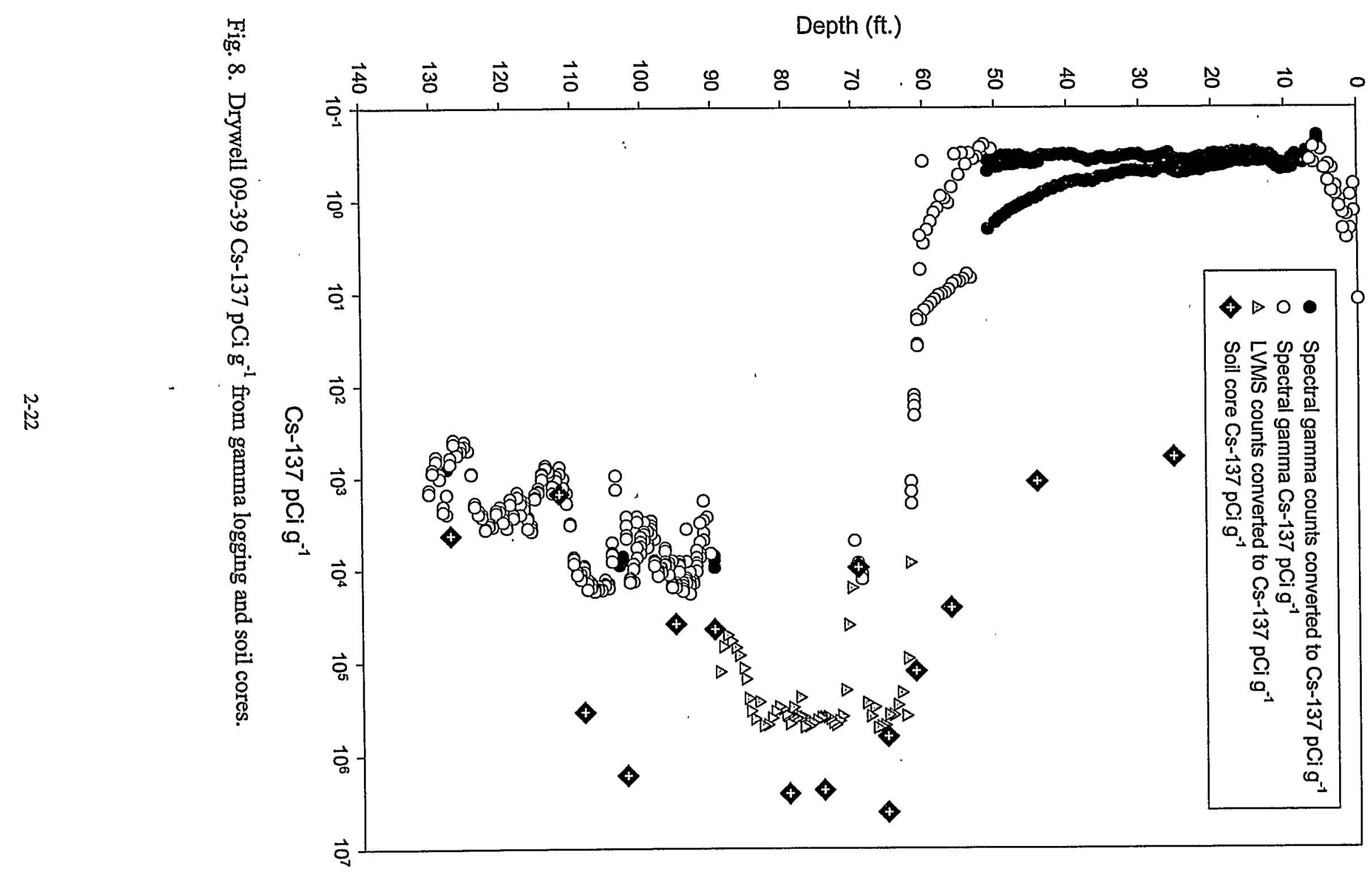


Incidentally, our inventory estimate for the plume under SX-108 is in accord with the estimate that Raymond and Shdo computed for that tank. If our inventory estimate for SX-115 is not correct, this must be owing to some misinterpretation of the old gamma logging data. The gamma logging in the SX-115 laterals was done some time ago, and the reports available to us are sketchy. Our attempts to cross-calibrate the soil sample Cs-137 data from the SX-115 cores with kriging predictions at those same locations based on the nearby gamma logging data did not yield a plausible pattern (Figure 9 ).



Fig. 9. SX-115 soil core Cs-137 $\mathrm{pCi} \mathrm{g}^{-1}$ vs. kriging-predicted type $4 \mathrm{cps}$.

A second feature of our inventory estimates that may appear odd is the near constancy of the inventory under some tanks for different choices of threshold, except for the very hottest thresholds (above $10^{5} \mathrm{pCi} / \mathrm{g}$ ). The explanation of this pattern is very straight forward. The inventory is dominated by a very few 
cells with very high counts. The data from the hottest cells are many orders of magnitude hotter than the data from almost all the other cells. The hotspots that the kriging estimates construct around these hot data cells are a relatively small volume. So the kriging estimates, like the data themselves, involve a relatively small number of cells that are many orders of magnitude hotter than rest of the volume. As a simple arithmetic consequence, the total inventory in all the cells outside this hot volume is negligible compared to the inventory in the hot volume.

\subsection{Prediction Error Variance for the Inventory}

Unlike the point estimates of inventory, the prediction error variance estimates for the inventory are very sensitive to assumptions, and are sensitive to the values used for process variance and measurement error variance. Furthermore, the jackknife cross-validation procedure for adjusting the $e$-folding distance parameters to fit the point estimates to the data does not enforce any fit of the prediction error variance. For this reason, we do not consider the prediction error variances reported here to be robust, though they were calculated from operations on bycell prediction error variances that were computed with a mathematically correct formula, and returned values that are not implausible. More modeling work, of a more complicated nature than was done here, would be needed to attain a higher level of security in the prediction error variance estimates. In particular, a strongly smoothed plume dispersion and transport model should be used to "detrend" the data, to obtain a more normal distribution of residuals that could be kriged, thus allowing for variation in the spatially correlated process on either side of the local process mean, and also allowing for a considerably smaller process variance (and hence for smaller prediction error variance) since a good fraction of the variance of the data will be accounted for by the "trend." Further, we recommend for future more refined estimation of the prediction error variance of the inventories, that the prediction error variances be re-calibrated by jack-knife cross-validation of the prediction error variance itself.

One pattern in the prediction error variances we report that may seem puzzling, is, in fact, readily explained. It will be noted in the tables of results, that as one lowers the threshold concentration that defines the volume of interest, the point estimate of the inventory does not increase much once the threshold is below about $10^{5}$, though the volume included goes up appreciably, and the prediction error variance goes up dramatically. Indeed the coefficient of variation, which is the standard deviation expressed as a fraction of point estimate, increases with 
the number of cells. At first this may seem to be contrary to the elementary properties of the model for second-order stationary Gaussian process.

If an arbitrary volume is defined under this model, and we consider the process itself, unconditioned on any data, the expected inventory of that volume should be proportional to the number of cells: it is, in fact, $m \mu$, where $m$ is the number of cells in the volume, and $\mu$ is the process mean. Once the number of cells defines a volume of large enough extent (compared to the $e$-folding distance) to average out the effect of spatial correlation, the variance of the inventory for the volume will be proportional to $m \sigma^{2}$, where $\sigma^{2}$ is the process variance. Thus the standard deviation of the inventory for the volume will be $\sigma \sqrt{m}$, and so expressing the standard deviation of the inventory as a fraction of the inventory (coefficient of variation) gives $\frac{\sigma}{\mu \sqrt{m}}$, which declines as the number of cells increases, going to zero in the limit.

So why do we observe an increasing coefficient of variation with increasing volume in the tables in Appendix 3? Bear in mind that the volumes for which we compute inventories are not randomly or arbitrarily located in the field of the process. The volumes are defined as collections of cells that exceed a defined threshold, so they are invariably centered on hotspots, and as the threshold is lowered and the number of cells included increases, the new cells being added to the volume are cooler and cooler. Thus, for volumes specified in this way, the inventory does not increase in proportion to the number of cells. In choosing our model to predict low values when far from hot data, we set the process mean at "background" with is also the mode of the lowest data values. Therefore, any kriged hotspot must be centered on data, and therefore at the hotspot center the prediction error variance must be low (because of the proximity to data). As the threshold is lowered, and cooler cells are included in the volume, a large fraction of the cells being added will be cells that are farther from data, and thus are cells with a larger prediction error variance than the point prediction for the core of the hotspot. Furthermore, if the typical spatial scale of a hotspot is similar to the $e$-folding distance, which is almost inevitable from the way we determined the value of that parameter, there will be considerable spatial correlation in error variation (contrary to the limiting assumption in the unconditional scenario), so the prediction error variance in the inventory will increase more than linearly with the sum of the prediction error variances of the cells (recall that the number of off-diagonal elements in the covariance matrix goes up almost with the square of the number of cells). For all these reasons, the square root of the prediction error variance of the inventory expressed as a fraction of the inventory can increase 
substantially as the number of cells in this selected volume increases: the inventory can increase much less than linearly with the number of cells, and the prediction error variance of the inventory can increase much more than linearly with the number of cells.

\section{FUTURE DIRECTIONS}

The gamma monitoring data set for SX farm is unusually rich. For the 15 tanks in this farm, we have data from 99 vertical far-perimeter drywells positioned the way most monitoring drywells are in the 200 area, plus 24 lateral drywells, plus soil sample data from one of the vertical drywells, and 15 soil cores from the tank close-perimeter (closer to the tank than the vertical drywells). For most of the vertical far-perimeter drywells, we have recent spectral gamma data.

We have gone to some effort to cross calibrate among readings from different gross gamma instruments (that differ in their sensitivity and shielding), and spectral gamma, spectral gamma Cs-137, and Cs-137 measurements from soil core samples. With this cross calibration, and decay correction, we have merged gross gamma logging data, and soil core sample Cs-137 measurements from earlier periods, to complete the data for locations that do not have recent spectral gamma measurements (or where the spectral gamma saturated).

Most of the other tank farms do not have similar data richness. The gamma monitoring data for most farms is confined to vertical drywells. Our preliminary results have already shown that the lateral data in SX farm make a considerable difference to the reconstructed plume and the estimate of plume inventory. Figure 10 shows a 6-tank subset of the farm, viewed from the East, with tank 108 in the center front. The lateral wells show as dark green horizontal lines under some of the tanks. The $e^{6} \mathrm{cps}$ (in probe type 4 units) iso-surface of the reconstruction of the plume from tanks 108 and 109 shows as the irregular shape. The reconstruction based just on the vertical drywell data appears as 4 disconnected opaque gray blobs; the transparent purple extension, that connects all the blobs into a single larger plume is the reconstruction that includes the lateral data with the vertical drywell data. 


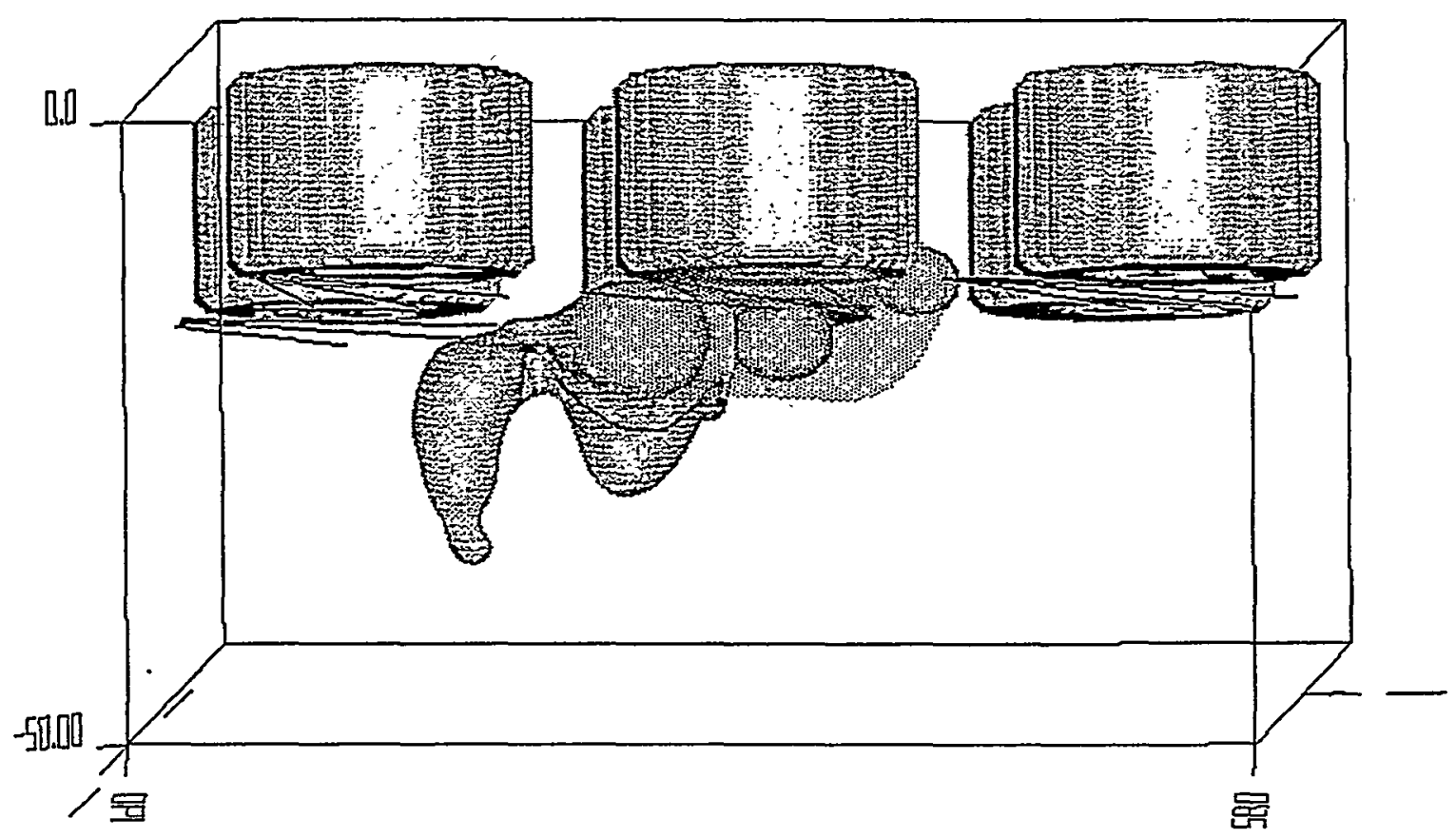

Fig 10. Six-tank subset of SX tank farm, east view.

\subsection{Calibrating Plume Estimates for Data Sets Without Laterals}

The composite Cs-137/gamma data from SX farm show definite contamination plumes under 5 of the tanks: 107, 108, 109, 112 and 115. For all 5 of these tanks, we have vertical drywell and lateral drywell data. For two of these tanks (108 and 115) we also have tank close-perimeter soil core sample data. This presents an opportunity to test the power and sensitivity of vertical drywell logging alone as a means to detect and quantify contamination plumes. In such an exercise, the geostatistical plume reconstruction from the merged data set, including laterals and perimeter soil cores, would be used as "ground truth," to investigate how accurately the plume can be reconstructed from vertical drywell data alone.

We have already noted, qualitatively, that the large plume under tanks 108 and 109 shows up clearly in the vertical drywell data (though it looks different when the lateral data are added). The smaller plumes under tanks 107 and 115 are not clearly delineated by the vertical drywell data, and need lateral and/or perimeter soil core data, closer to the actual plume, in order for the full extent of the plume to be evident. 
The point of a plume reconstruction calibration analysis would be to refine and quantify these observations, (1) to determine how large a plume inventory has to be in order for it to be detected with confidence using vertical drywell data alone, and (2) to calibrate the difference in plume inventory estimates using all the data versus vertical drywell data alone, to establish the reliability of a corrected estimate based on vertical drywell data alone.

These calibrations would be very important for interpreting the geostatistics from other tank farms which have only vertical drywell data.

\section{REFERENCES}

Cressie, N. 1991. Statistics for Spatial Data. John Wiley \& Sons, N.Y.

Goodman, D. 1999. Documentation of Geostatistical Approach for the Analysis of SX-Farm Vadose Zone Gamma Data. September 27, 1999, report to MACTEC Inc., under agreement 3751.119.376 with Montana State University.

Kitanidis, P.K. 1997. Introduction to Geostatistics. Cambridge University Press. Cambridge.

Ng, E.G., and B.W. Peyton. 1993. Block sparse Cholesky algorithms on advanced uniprocessor computers. SIAM-Journal of Scientific Computing 1034-1056.

Raymond, J.R., and E.G. Shdo. 1966. Characterization of Subsurface Contamination in the SX Tank Farm. BNWL-CC-701, Battelle-Northwest, Richland, Washington.

Ripley, B.D. 1981. Spatial Statistics. John Wiley \& Sons, N.Y.

Wiener, N. 1949. Extrapolation, Interpolation, and Smoothing of Stationary Time Series. Technology Press, MIT, and John Wiley \& Sons, N.Y. 


\section{APPENDIX 3. RESULTS OF THE SX INVENTORY CALCULATION}

Kriging point predictions in $\mathrm{Cs}-137 \mathrm{pCi} \mathrm{g}^{-1}$ were estimated at one meter spacings on a three dimensional grid filling the prediction volume (Fig. 1, Appendix 1.), where grid point $(0,0,0)$ was located at easting $566745 \mathrm{~m}$, northing $134145 \mathrm{~m}$, and a depth of $50 \mathrm{~m}$. Individual tank inventories were calculated from the kriging point predictions falling within the soil subvolume associated with each tank (Fig. 1, Appendix 1.). However, kriging predictions assigned to a soil subvolume, but actually located within a storage tank or lateral caisson, were ignored.

The Cs-137 inventory associated with each tank was broken down by soil volumes exceeding eight respective limits, $5 * 10^{1}, 5^{*} 10^{2}, 5^{*} 10^{3}, 5^{*} 10^{4}, 5^{*} 10^{5}, 5^{*} 10^{6}, 5^{*} 10^{7}$, and $5^{*} 10^{8}$ $\mathrm{pCi} \mathrm{g}^{-1}$. Individual tank inventories exceeding these thresholds were further broken down into 17 depth increments consisting of a $2.5 \mathrm{~m}$ layer from the surface of the prediction volume to a depth of $2.5 \mathrm{~m}$ and $16-3 \mathrm{~m}$ depth increments from $2.5 \mathrm{~m}$ to $50.5 \mathrm{~m}$. Given that the surface of the SX farm is not level, and given that the surface of the prediction volume was set at the surface elevation of the highest drywell in the SX-farm, the 0.0 to $2.5 \mathrm{~m}$ depth increment is somewhat artificial, containing a number of point predictions that are actually aboveground. However, relatively little Cs-137 contamination was predicted within this surface layer.

Additionally, given that the vertical separation between the surface elevation of the highest and lowest drywells is approximately $2.2 \mathrm{~m}$, all artifactual predictions are restricted to this layer.

In calculating Cs-137 inventories, each kriging point prediction was considered to be at the center of a one cubic meter soil volume with an average Cs-137 concentration equal to the point prediction. Note that the tank subvolume boundaries in Fig. 1, Appendix 1. were located half way between neighboring vertical planes of kriging point predictions to accommodate this geometry. Given a soil bulk density of $1.8 \mathrm{~g} \mathrm{~cm}^{-3}$ at the SX tank farm (Raymond and Shdo, 1966), each point prediction in $\mathrm{pCi} \mathrm{g}^{-1}$ was converted to a total Cs-137 activity in pCi for a one cubic meter soil volume by multiplying by $1.8 * 10^{6}$. Once this conversion was made, individual Cs 137 inventories were calculated simply by summing the total activities of one cubic meter cubes. Given the conversion from $\mathrm{pCi} \mathrm{g}^{-1}$ to $\mathrm{pCi} \mathrm{m}^{-3}$ of soil, kriging prediction variances were scaled to one cubic meter soil volumes by multiplying by $\left(1.8 * 10^{6}\right)^{2}$. Confidence intervals associated with individual inventories were calculated as described in Appendix 2. All inventories and their associated confidence intervals were decay corrected to January, 2000 values.

Cs-137 inventories over the entire soil volume associated with each SX storage tank are summarized in Table 1. Inventories broken down by depth increments are presented for individual tank volumes in Tables 2 through 16. Vis5D visualizations of the plumes exceeding seven activity thresholds (no kriging point predictions were greater than $5^{*} 10^{8} \mathrm{pCi} \mathrm{g}^{-1}$ ) are plotted in Figures 1 though 7. 
Table 1. sx tank farm inventory of soil volumes, total activities and upper 95\% confidence limit, exceeding 8 activity thresholds.

\begin{tabular}{|c|c|c|c|c|}
\hline Tank & $\begin{array}{l}\text { Threshold } \\
(\mathrm{pCi} / \mathrm{g})\end{array}$ & $\begin{array}{l}\text { Soil } \\
\text { Volume }\left(\mathrm{m}^{3}\right)\end{array}$ & $\begin{array}{l}\text { Total } \\
\text { (pci) }\end{array}$ & $\begin{array}{l}\text { Upper } 95 \% \\
\text { (pCi) }\end{array}$ \\
\hline$S X-101$ & $5 \times 10^{1}$ & 73 & $9.07076 \mathrm{E}+09$ & $2.09415 E+10$ \\
\hline $5 x-101$ & $5 \times 10^{2}$ & 0 & 0 & 0 \\
\hline $5 x-101$ & $5 \times 10^{3}$ & 0 & 0 & 0 \\
\hline $5 x-101$ & $5 \times 10^{4}$ & 0 & 0 & 0 \\
\hline $5 x-101$ & $5 \times 10^{5}$ & 0 & 0 & 0 \\
\hline $5 x-101$ & $5 \times 10^{6}$ & 0 & 0 & 0 \\
\hline $5 x-101$ & $5 \times 10^{7}$ & 0 & 0 & 0 \\
\hline $5 x-101$ & $5 \times 10^{8}$ & 0 & 0 & 0 \\
\hline $5 x-102$ & $5 \times 10^{1}$ & 0 & 0 & 0 \\
\hline $5 x-102$ & $5 \times 10^{2}$ & 0 & 0 & 0 \\
\hline $5 x-102$ & $5 \times 10^{3}$ & 0 & 0 & 0 \\
\hline$s x-102$ & $5 \times 10^{4}$ & 0 & 0 & 0 \\
\hline $5 x-102$ & $5 \times 10^{5}$ & 0 & 0 & 0 \\
\hline $5 x-102$ & $5 \times 10^{6}$ & 0 & 0 & 0 \\
\hline $5 x-102$ & $5 \times 10^{7}$ & 0 & 0 & 0 \\
\hline$s x-102$ & $5 \times 10^{8}$ & 0 & 0 & 0 \\
\hline $5 x-103$ & $5 \times 10^{1}$ & 0 & 0 & 0 \\
\hline$s x-103$ & $5 \times 10^{2}$ & 0 & 0 & 0 \\
\hline$s X-103$ & $5 \times 10^{3}$ & 0 & 0 & 0 \\
\hline$S X-103$ & $5 \times 10^{4}$ & 0 & 0 & 0 \\
\hline $5 X-103$ & $5 \times 10^{5}$ & 0 & 0 & 0 \\
\hline$S x-103$ & $5 \times 10^{6}$ & 0 & 0 & 0 \\
\hline$S x-103$ & $5 \times 10^{7}$ & 0 & 0 & 0 \\
\hline$S X-103$ & $5 \times 10^{8}$ & 0 & 0 & 0 \\
\hline$S X-104$ & $5 \times 10^{2}$ & 3 & $2.65346 \mathrm{E}+08$ & $1.55831 \mathrm{E}+09$ \\
\hline$s x-104$ & $5 \times 10^{2}$ & 0 & 0 & 0 \\
\hline $5 X-104$ & $5 \times 10^{3}$ & 0 & 0 & 0 \\
\hline $5 X-104$ & $5 \times 10^{4}$ & 0 & 0 & 0 \\
\hline$S X-104$ & $5 \times 10^{5}$ & 0 & 0 & 0 \\
\hline$S X-104$ & $5 \times 10^{6}$ & 0 & 0 & 0 \\
\hline$S X-104$ & $5 \times 10^{7}$ & 0 & 0 & 0 \\
\hline $5 X-104$ & $5 \times 10^{8}$ & 0 & 0 & 0 \\
\hline$s x-105$ & $5 \times 10^{1}$ & 26 & $5.51622 \mathrm{E}+09$ & $1.33633 \mathrm{E}+10$ \\
\hline$S x-105$ & $5 \times 10^{2}$ & 0 & 0 & 0 \\
\hline$S X-105$ & $5 \times 10^{3}$ & 0 & 0 & 0 \\
\hline$S X-105$ & $5 \times 10^{4}$ & 0 & 0 & 0 \\
\hline$s X-105$ & $5 \times 10^{5}$ & 0 & 0 & 0 \\
\hline$S x-105$ & $5 \times 10^{6}$ & 0 & 0 & 0 \\
\hline$s X-105$ & $5 \times 10^{7}$ & 0 & 0 & 0 \\
\hline$S X-105$ & $5 \times 10^{8}$ & 0 & 0 & 0 \\
\hline$s X-106$ & $5 \times 10^{1}$ & 0 & 0 & 0 \\
\hline $5 x-106$ & $5 \times 10^{2}$ & 0 & 0 & 0 \\
\hline SX-106 & $5 \times 10^{3}$ & 0 & 0 & 0 \\
\hline$S x-106$ & $5 \times 10^{4}$ & 0 & 0 & 0 \\
\hline $5 x-106$ & $5 \times 10^{5}$ & 0 & 0 & 0 \\
\hline $5 x-106$ & $5 \times 10^{6}$ & 0 & 0 & 0 \\
\hline $5 X-106$ & $5 \times 10^{7}$ & 0 & 0 & 0 \\
\hline $5 x-106$ & $5 \times 10^{8}$ & 0 & 0 & 0 \\
\hline$s x-107$ & $5 \times 10^{1}$ & 2244 & $8.02620 \mathrm{E}+15$ & $1.48792 \mathrm{E}+16$ \\
\hline $5 X-107$ & $5 \times 10^{2}$ & 1462 & $8.02597 \mathrm{E}+15$ & $1.48787 E+16$ \\
\hline SX-107 & $5 \times 10^{3}$ & 937 & $8.02436 \mathrm{E}+15$ & $1.48748 \mathrm{E}+16$ \\
\hline $5 X-107$ & $5 \times 10^{4}$ & 578 & $8.01356 \mathrm{E}+15$ & $1.48508 \mathrm{E}+16$ \\
\hline $5 x-107$ & $5 \times 10^{5}$ & 283 & $7.93281 \mathrm{E}+15$ & $1.46781 \mathrm{E}+16$ \\
\hline$s x-107$ & $5 \times 10^{6}$ & 108 & $7.40611 \mathrm{E}+15$ & $1.36282 \mathrm{E}+16$ \\
\hline$s X-107$ & $5 \times 10^{7}$ & 24 & $5.42652 \mathrm{E}+15$ & $9.64043 \mathrm{E}+15$ \\
\hline$s x-107$ & $5 \times 10^{8}$ & 0 & 0 & 0 \\
\hline$s x-108$ & $5 \times 10^{1}$ & 5022 & $1.43107 \mathrm{E}+16$ & $3.55814 E+16$ \\
\hline$s x-108$ & $5 \times 10^{2}$ & 3490 & $1.43102 \mathrm{E}+16$ & $3.55803 \mathrm{E}+16$ \\
\hline$s x-108$ & $5 \times 10^{3}$ & 2466 & $1.43072 \mathrm{E}+16$ & $3.55734 \mathrm{E}+16$ \\
\hline $5 x-108$ & $5 \times 10^{4}$ & 1608 & $1.42806 \mathrm{E}+16$ & $3.55140 \mathrm{E}+16$ \\
\hline
\end{tabular}


HNF-5782 Rev.0

Table 1. Continued.

\begin{tabular}{|c|c|c|c|c|}
\hline Tank & $\begin{array}{l}\text { Threshold } \\
(\mathrm{pCi} / \mathrm{g})\end{array}$ & $\begin{array}{c}\text { Soil } \\
\text { Volume }\left(\mathrm{m}^{3}\right)\end{array}$ & $\begin{array}{l}\text { Total } \\
\text { (pci) }\end{array}$ & $\begin{array}{l}\text { Upper } 95 \% \\
\text { (pci) }\end{array}$ \\
\hline $5 x-108$ & $5 \times 10^{5}$ & 903 & $1.40763 \mathrm{E}+16$ & $3.50338 \mathrm{E}+16$ \\
\hline $5 X-108$ & $5 \times 10^{6}$ & 389 & $1.24209 \mathrm{E}+16$ & $3.11132 \mathrm{E}+16$ \\
\hline $5 X-108$ & $5 \times 10^{7}$ & 2 & $1.70049 \mathrm{E}+14$ & $5.43278 \mathrm{E}+14$ \\
\hline SX-108 & $5 \times 10^{8}$ & 0 & 0 & 0 \\
\hline $5 x-109$ & $5 \times 10_{2}^{1}$ & 7949 & $7.22521 E+14$ & $2.32289 \mathrm{E}+15$ \\
\hline $5 X-109$ & $5 \times 10^{2}$ & 4966 & $7.21634 \mathrm{E}+14$ & $2.32027 E+15$ \\
\hline $5 x-109$ & $5 \times 10^{3}$ & 2813 & $7.15346 \mathrm{E}+14$ & $2.30162 E+15$ \\
\hline $5 x-109$ & $5 \times 10^{4}$ & 1236 & $6.68361 E+14$ & $2.15829 E+15$ \\
\hline $5 x-109$ & $5 \times 10^{5}$ & 223 & $4.07739 E+14$ & $1.35166 \mathrm{E}+15$ \\
\hline $5 X-109$ & $5 \times 10^{6}$ & 0 & 0 & 0 \\
\hline $5 X-109$ & $5 \times 10^{7}$ & 0 & 0 & 0 \\
\hline $5 x-109$ & $5 \times 10^{8}$ & 0 & 0 & 0 \\
\hline $5 x-110$ & $5 \times 10^{1}$ & 66 & $1.97603 E+12$ & $1.25766 \mathrm{E}+13$ \\
\hline $5 x-110$ & $5 \times 10^{2}$ & 29 & $1.96597 \mathrm{E}+12$ & $1.25304 \mathrm{E}+13$ \\
\hline$s x-110$ & $5 \times 10^{3}$ & 11 & $1.91158 \mathrm{E}+12$ & $1.22570 \mathrm{E}+13$ \\
\hline $5 X-110$ & $5 \times 10^{4}$ & 4 & $1.76547 \mathrm{E}+12$ & $1.14944 \mathrm{E}+13$ \\
\hline$S X-110$ & $5 \times 10^{5}$ & 1 & $8.92941 E+11$ & $6.59409 \mathrm{E}+12$ \\
\hline $5 X-110$ & $5 \times 10^{6}$ & 0 & 0 & 0 \\
\hline sX -110 & $5 \times 10^{7}$ & 0 & 0 & 0 \\
\hline $5 x-110$ & $5 \times 10^{8}$ & 0 & 0 & 0 \\
\hline$s x-111$ & $5 \times 10^{1}$ & 381 & $2.34596 \mathrm{E}+11$ & $1.14707 \mathrm{E}+12$ \\
\hline $5 X-111$ & $5 \times 10^{2}$ & 58 & $1.60544 \mathrm{E}+11$ & $8.50179 E+11$ \\
\hline$S X-111$ & $5 \times 10^{3}$ & 4 & $4.36899 \mathrm{E}+10$ & $2.56878 \mathrm{E}+11$ \\
\hline$s X-111$ & $5 \times 10^{4}$ & 0 & 0 & 0 \\
\hline$s x-111$ & $5 \times 10^{5}$ & 0 & 0 & 0 \\
\hline $5 x-111$ & $5 \times 10^{6}$ & 0 & 0 & 0 \\
\hline$s x-111$ & $5 \times 10^{7}$ & 0 & 0 & 0 \\
\hline SX-1II & $5 \times 10^{8}$ & 0 & 0 & 0 \\
\hline $5 x-112$ & $5 \times 10^{1}$ & 2922 & $1.80110 \mathrm{E}+13$ & $9.50258 \mathrm{E}+13$ \\
\hline $5 x-112$ & $5 \times 10^{2}$ & 1141 & $1.75140 \mathrm{E}+13$ & $9.32021 \mathrm{E}+13$ \\
\hline $5 X-112$ & $5 \times 10^{3}$ & 297 & $1.52622 E+13$ & $8.32576 \mathrm{E}+13$ \\
\hline$S x-112$ & $5 \times 10^{4}$ & 53 & $9.44060 \mathrm{E}+12$ & $5.51191 \mathrm{E}+13$ \\
\hline$s x-112$ & $5 \times 10^{5}$ & 0 & 0 & 0 \\
\hline$s x-112$ & $5 \times 10^{6}$ & 0 & 0 & 0 \\
\hline$s x-112$ & $5 \times 10^{7}$ & 0 & 0 & 0 \\
\hline$s x-112$ & $5 \times 10^{8}$ & 0 & 0 & 0 \\
\hline $5 x-113$ & $5 \times 10^{1}$ & 0 & 0 & 0 \\
\hline$S x-113$ & $5 \times 10^{2}$ & 0 & 0 & 0 \\
\hline$s x-113$ & $5 \times 10^{3}$ & 0 & 0 & 0 \\
\hline$s x-113$ & $5 \times 10^{4}$ & 0 & 0 & 0 \\
\hline $5 x-113$ & $5 \times 10^{5}$ & 0 & 0 & 0 \\
\hline $5 x-113$ & $5 \times 10^{6}$ & 0 & 0 & 0 \\
\hline $5 x-113$ & $5 \times 10^{7}$ & 0 & 0 & 0 \\
\hline $5 x-113$ & $5 \times 10^{8}$ & 0 & 0 & 0 \\
\hline $5 x-114$ & $5 \times 10^{1}$ & 0 & 0 & 0 \\
\hline$s x-114$ & $5 \times 10_{2}^{2}$ & 0 & 0 & 0 \\
\hline$s X-114$ & $5 \times 10^{3}$ & 0 & 0 & 0 \\
\hline$s x-114$ & $5 \times 10^{4}$ & 0 & 0 & 0 \\
\hline$s x-114$ & $5 \times 10^{5}$ & 0 & 0 & 0 \\
\hline$s x-114$ & $5 \times 10^{6}$ & 0 & 0 & 0 \\
\hline $5 x-114$ & $5 \times 10^{7}$ & 0 & 0 & 0 \\
\hline$s x-114$ & $5 \times 10^{8}$ & -0 & 0 & 0 \\
\hline$s x-115$ & $5 \times 10^{1}$ & 813 & $4.13363 \mathrm{E}+12$ & $1.76788 \mathrm{E}+13$ \\
\hline$s x-115$ & $5 \times 10^{2}$ & 289 & $3.99028 E+12$ & $1.73118 \mathrm{E}+13$ \\
\hline$s x-115$ & $5 \times 10^{3}$ & 82 & $3.47943 E+12$ & $1.58659 \mathrm{E}+13$ \\
\hline$S X-115$ & $5 \times 10_{5}^{4}$ & 10 & $1.89946 \mathrm{E}+12$ & $9.95216 \mathrm{E}+12$ \\
\hline$s x-115$ & $5 \times 10^{5}$ & 0 & 0 & 0 \\
\hline$s X-115$ & $5 \times 10^{6}$ & 0 & 0 & 0 \\
\hline$s x-115$ & $5 \times 10^{7}$ & 0 & 0 & 0 \\
\hline$s X-115$ & $5 \times 10^{8}$ & 0 & 0 & 0 \\
\hline
\end{tabular}


Table 2. SX-10I inventory of soil volumes, total activities and upper 95\% confidence limit, exceeding 8 activity thresholds over 17 depth increments.

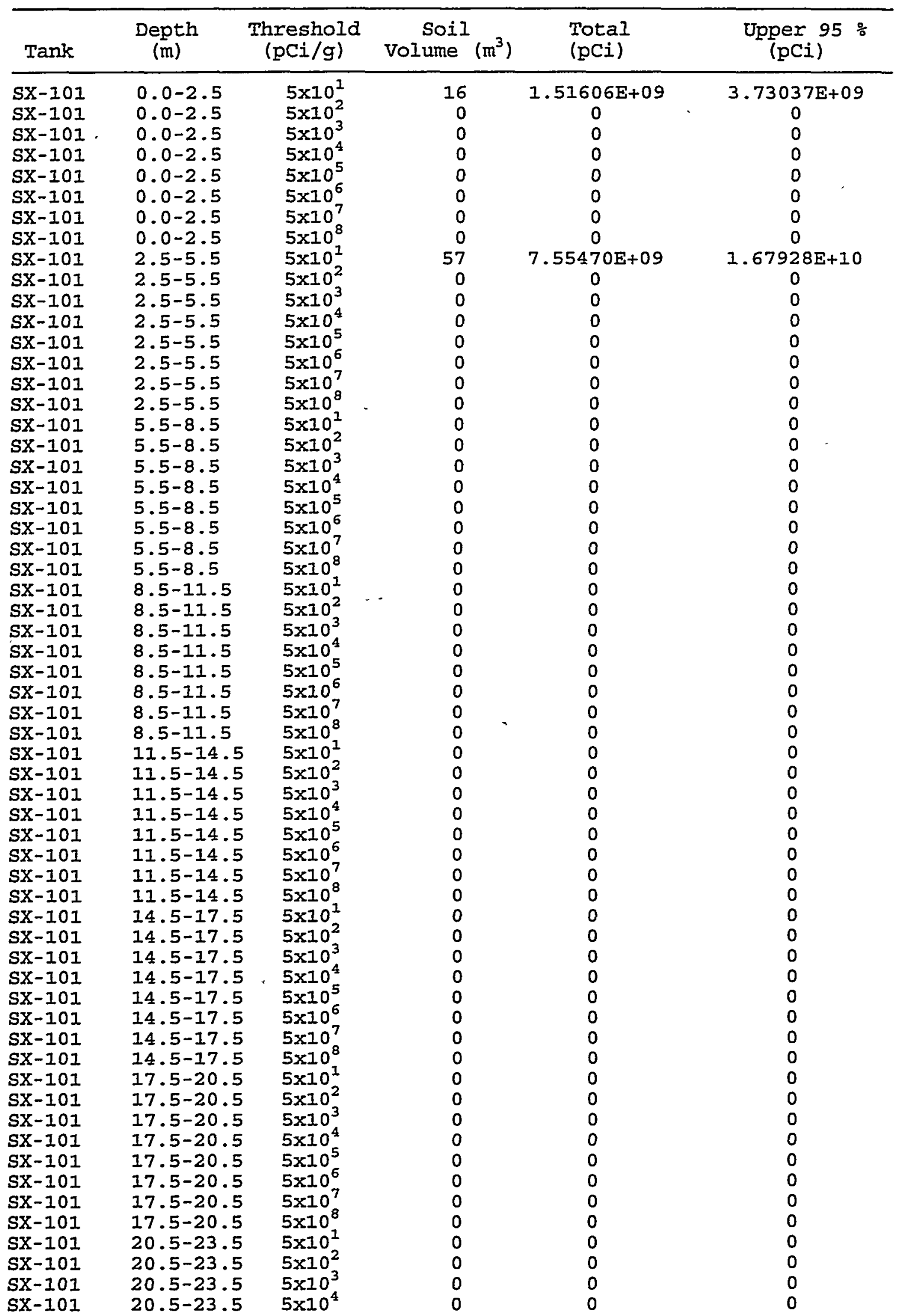


Table 2. Continued.

\begin{tabular}{|c|c|c|c|c|c|}
\hline Tank & $\underset{(\mathfrak{m})}{\operatorname{Depth}}$ & $\begin{array}{l}\text { Threshold } \\
\text { (pCi/g) }\end{array}$ & $\begin{array}{c}\text { Soil } \\
\text { Volume }\left(\mathrm{m}^{3}\right)\end{array}$ & $\begin{array}{l}\text { Total } \\
\text { (pCi) }\end{array}$ & $\begin{array}{l}\text { Upper } 95 \% \\
\text { (pCi) }\end{array}$ \\
\hline $5 X-101$ & $20.5-23.5$ & $5 \times 10^{5}$ & 0 & 0 & 0 \\
\hline$S X-101$ & $20.5-23.5$ & $5 \times 10^{6}$ & 0 & 0 & 0 \\
\hline $5 X-101$ & $20.5-23.5$ & $5 \times 10^{7}$ & 0 & 0 & 0 \\
\hline $5 X-101$ & $20.5-23.5$ & $5 \times 10^{8}$ & 0 & 0 & 0 \\
\hline $5 x-101$ & $23.5-26.5$ & $5 \times 10^{1}$ & 0 & 0 & 0 \\
\hline $5 X-101$ & $23.5-26.5$ & $5 \times 10_{3}^{2}$ & 0 & 0 & 0 \\
\hline $5 x-101$ & $23.5-26.5$ & $5 \times 10^{3}$ & 0 & 0 & 0 \\
\hline $5 x-101$ & $23.5-26.5$ & $5 \times 10^{4}$ & 0 & 0 & 0 \\
\hline $5 X-101$ & $23.5-26.5$ & $5 \times 10^{5}$ & 0 & 0 & 0 \\
\hline $5 x-101$ & $23.5-26.5$ & $5 \times 10^{6}$ & 0 & 0 & 0 \\
\hline$s X-101$ & $23.5-26.5$ & $5 \times 10^{7}$ & 0 & 0 & 0 \\
\hline $5 x-101$ & $23.5-26.5$ & $5 \times 10^{8}$ & 0 & 0 & 0 \\
\hline $5 X-101$ & $26.5-29.5$ & $5 \times 10^{1}$ & 0 & 0 & 0 \\
\hline $5 x-101$ & $26.5-29.5$ & $5 \times 10_{2}^{2}$ & 0 & 0 & 0 \\
\hline$s x-101$ & $26.5-29.5$ & $5 \times 10^{3}$ & 0 & 0 & 0 \\
\hline $5 x-101$ & $26.5-29.5$ & $5 \times 10^{4}$ & 0 & 0 & 0 \\
\hline$s x-101$ & $26.5-29.5$ & $5 \times 10^{5}$ & 0 & 0 & 0 \\
\hline$s x-101$ & $26.5-29.5$ & $5 \times 10^{6}$ & 0 & 0 & 0 \\
\hline$s x-101$ & $26.5-29.5$ & $5 \times 10^{7}$ & 0 & 0 & 0 \\
\hline$S X-101$ & $26.5-29.5$ & $5 \times 10^{8}$ & 0 & 0 & 0 \\
\hline$s x-101$ & $29.5-32.5$ & $5 \times 10^{1}$ & 0 & 0 & 0 \\
\hline$s X-101$ & $29.5-32.5$ & $5 \times 10^{2}$ & 0 & 0 & 0 \\
\hline$s x-101$ & $29.5-32.5$ & $5 \times 10^{3}$ & 0 & 0 & 0 \\
\hline $5 x-101$ & $29.5-32.5$ & $5 \times 10^{4}$ & $0^{-}$ & 0 & 0 \\
\hline $5 X-101$ & $29.5-32.5$ & $5 \times 10^{5}$ & 0 & 0 & 0 \\
\hline $5 X-101$ & $29.5-32.5$ & $5 \times 10^{6}$ & 0 & 0 & 0 \\
\hline $5 X-101$ & $29.5-32.5$ & $5 \times 10^{7}$ & 0 & 0 & 0 \\
\hline $5 X-101$ & $29.5-32.5$ & $5 \times 10^{8}$ & 0 & 0 & 0 \\
\hline$S X-101$ & $32.5-35.5$ & $5 \times 10^{1}$ & 0 & 0 & 0 \\
\hline$S X-101$ & $32.5-35.5$ & $5 \times 10^{2}$ & 0 & 0 & 0 \\
\hline $5 x-101$ & $32.5-35.5$ & $5 \times 10^{3}$ & 0 & 0 & 0 \\
\hline$s x-101$ & $32.5-35.5$ & $5 \times 10^{4}$ & 0 & 0 & 0 \\
\hline $5 x-101$ & $32.5-35.5$ & $5 \times 10^{5}$ & 0 & 0 & 0 \\
\hline $5 X-10 I$ & $32.5-35.5$ & $5 \times 10^{6}$ & 0 & 0 & 0 \\
\hline $5 X-101$ & $32.5-35.5$ & $5 \times 10^{7}$ & 0 & 0 & 0 \\
\hline $5 X-101$ & $32.5-35.5$ & $5 \times 10^{8}$ & 0 & 0 & 0 \\
\hline $5 X-101$ & $35.5-38.5$ & $5 \times 10^{1}$ & 0 & 0 & 0 \\
\hline$s x-101$ & $35.5-38.5$ & $5 \times 10^{2}$ & 0 & 0 & 0 \\
\hline $5 X-101$ & $35.5-38.5$ & $5 \times 10^{3}$ & 0 & 0 & 0 \\
\hline $5 X-10 I$ & $35.5-38.5$ & $5 \times 10^{4}$ & 0 & 0 & 0 \\
\hline $5 x-101$ & $35.5-38.5$ & $5 \times 10^{5}$ & 0 & 0 & 0 \\
\hline $5 x-101$ & $35.5-38.5$ & $5 \times 10^{6}$ & 0 & 0 & 0 \\
\hline $5 x-101$ & $35.5-38.5$ & $5 \times 10^{7}$ & 0 & 0 & 0 \\
\hline$S X-10 I$ & $35.5-38.5$ & $5 \times 10^{8}$ & 0 & 0 & 0 \\
\hline$s x-101$ & $38.5-41.5$ & $5 \times 10^{1}$ & 0 & 0 & 0 \\
\hline$s x-101$ & $38.5-41.5$ & $5 \times 10^{2}$ & 0 & 0 & 0 \\
\hline $5 x-101$ & $38.5-41.5$ & $5 \times 10^{3}$ & 0 & 0 & 0 \\
\hline $5 X-101$ & $38.5-41.5$ & $5 \times 10^{4}$ & 0 & 0 & 0 \\
\hline$s x-101$ & $38.5-41.5$ & $5 \times 10^{5}$ & 0 & 0 & 0 \\
\hline$S X-101$ & $38.5-41.5$ & $5 \times 10^{6}$ & 0 & 0 & 0 \\
\hline$s X-101$ & $38.5-41.5$ & $5 \times 10^{7}$ & 0 & 0 & 0 \\
\hline$s x-101$ & $38.5-41.5$ & $5 \times 10^{8}$ & 0 & 0 & 0 \\
\hline$S X-101$ & $41.5-44.5$ & $5 \times 10^{1}$ & 0 & 0 & 0 \\
\hline$S X-10 I$ & $41.5-44.5$ & $5 \times 10^{2}$ & 0 & 0 & 0 \\
\hline$s x-101$ & $41.5-44.5$ & $5 \times 10^{3}$ & 0 & 0 & 0 \\
\hline$s x-101$ & $41.5-44.5$ & $5 \times 10^{4}$ & 0 & 0 & 0 \\
\hline$s x-101$ & $41.5-44.5$ & $5 \times 10^{5}$ & 0 & 0 & 0 \\
\hline$s X-101$ & $41.5-44.5$ & $5 \times 10^{6}$ & 0 & 0 & 0 \\
\hline$s X-101$ & $41.5-44.5$ & $5 \times 10^{7}$ & 0 & 0 & 0 \\
\hline$s X-10 I$ & $41.5-44.5$ & $5 \times 10^{8}$ & 0 & 0 & 0 \\
\hline $5 X-101$ & $44.5-47.5$ & $5 \times 10^{1}$ & 0 & 0 & 0 \\
\hline & & $5 \times 1$ & & & \\
\hline
\end{tabular}


HNF-5782 Rev.0

Table 2. Continued.

\begin{tabular}{cccccc}
\hline Tank & $\begin{array}{c}\text { Depth } \\
(\mathrm{m})\end{array}$ & $\begin{array}{c}\text { Threshold } \\
(\mathrm{pCi} / \mathrm{g})\end{array}$ & $\begin{array}{c}\text { Soil } \\
\text { Volume }\end{array}$ & $\begin{array}{c}\text { Total } \\
\text { (pCi) }\end{array}$ & $\begin{array}{c}\text { Upper 95 } \\
\text { (pCi) }\end{array}$ \\
\hline SX-101 & $44.5-47.5$ & $5 \times 10^{3}$ & 0 & 0 & 0 \\
SX-101 & $44.5-47.5$ & $5 \times 10^{4}$ & 0 & 0 & 0 \\
SX-101 & $44.5-47.5$ & $5 \times 10^{5}$ & 0 & 0 & 0 \\
SX-101 & $44.5-47.5$ & $5 \times 10^{6}$ & 0 & 0 & 0 \\
SX-101 & $44.5-47.5$ & $5 \times 10^{7}$ & 0 & 0 & 0 \\
SX-101 & $44.5-47.5$ & $5 \times 10^{8}$ & 0 & 0 & 0 \\
SX-101 & $47.5-50.5$ & $5 \times 10^{1}$ & 0 & 0 & 0 \\
SX-101 & $47.5-50.5$ & $5 \times 10^{2}$ & 0 & 0 & 0 \\
SX-101 & $47.5-50.5$ & $5 \times 10^{3}$ & 0 & 0 & 0 \\
SX-101 & $47.5-50.5$ & $5 \times 10^{4}$ & 0 & 0 & 0 \\
SX-101 & $47.5-50.5$ & $5 \times 10^{5}$ & 0 & 0 & 0 \\
SX-101 & $47.5-50.5$ & $5 \times 10^{6}$ & 0 & 0 & 0 \\
SX-101 & $47.5-50.5$ & $5 \times 10^{7}$ & 0 & 0 & 0 \\
SX-101 & $47.5-50.5$ & $5 \times 10^{8}$ & 0 & 0 & 0 \\
\hline
\end{tabular}


Table 3. Sx-102 inventory of soil volumes, total activities and upper 95\% confidence limit, exceeding 8 activity thresholds over 17 depth increments.

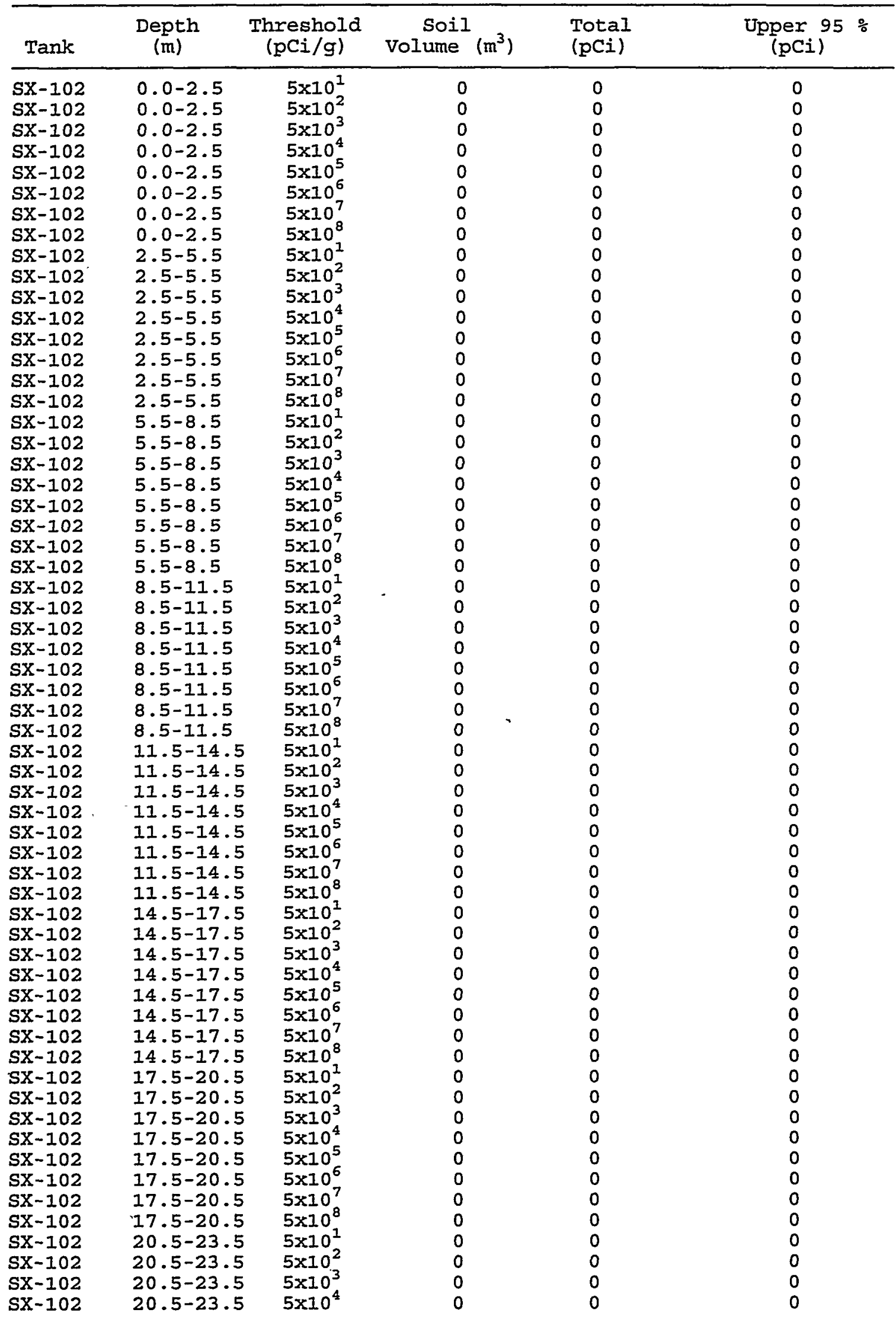


Table 3 . Continued.




HNF-5782 Rev.0

Table 3. Continued.

\begin{tabular}{cccccc}
\hline Tank & $\begin{array}{c}\text { Depth } \\
(\mathrm{m})\end{array}$ & $\begin{array}{c}\text { Threshold } \\
(\mathrm{pCi} / \mathrm{g})\end{array}$ & $\begin{array}{c}\text { Soil } \\
\text { Volume }\end{array}$ & $\begin{array}{c}\text { Total } \\
(\mathrm{pCi})\end{array}$ & $\begin{array}{c}\text { Upper } 95 \% \\
(\mathrm{pC})\end{array}$ \\
\hline $\mathrm{SX}-102$ & $44.5-47.5$ & $5 \times 10^{3}$ & 0 & 0 & 0 \\
$\mathrm{SX}-102$ & $44.5-47.5$ & $5 \times 10^{4}$ & 0 & 0 & 0 \\
$\mathrm{SX}-102$ & $44.5-47.5$ & $5 \times 10^{5}$ & 0 & 0 & 0 \\
$\mathrm{SX}-102$ & $44.5-47.5$ & $5 \times 10^{6}$ & 0 & 0 & 0 \\
$\mathrm{SX}-102$ & $44.5-47.5$ & $5 \times 10^{7}$ & 0 & 0 & 0 \\
$\mathrm{SX}-102$ & $44.5-47.5$ & $5 \times 10^{8}$ & 0 & 0 & 0 \\
$\mathrm{SX}-102$ & $47.5-50.5$ & $5 \times 10^{1}$ & 0 & 0 & 0 \\
$\mathrm{SX}-102$ & $47.5-50.5$ & $5 \times 10^{2}$ & 0 & 0 & 0 \\
$\mathrm{SX}-102$ & $47.5-50.5$ & $5 \times 10^{3}$ & 0 & 0 & 0 \\
$\mathrm{SX}-102$ & $47.5-50.5$ & $5 \times 10^{4}$ & 0 & 0 & 0 \\
$\mathrm{SX}-102$ & $47.5-50.5$ & $5 \times 10^{5}$ & 0 & 0 & 0 \\
$\mathrm{SX}-102$ & $47.5-50.5$ & $5 \times 10^{6}$ & 0 & 0 & 0 \\
$\mathrm{SX}-102$ & $47.5-50.5$ & $5 \times 10^{7}$ & 0 & 0 & \\
$\mathrm{SX}-102$ & $47.5-50.5$ & $5 \times 10^{8}$ & 0 & 0 & \\
\hline
\end{tabular}


Table 4. SX-103 inventory of soil volumes, total activities and upper 95\% confidence limit, exceeding 8 activity thresholds over 17 depth increments.

\begin{tabular}{|c|c|c|c|c|c|}
\hline Tank & $\begin{array}{l}\text { Depth } \\
\text { (m) }\end{array}$ & $\begin{array}{l}\text { Threshold } \\
(\mathrm{pCi} / \mathrm{g})\end{array}$ & $\begin{array}{l}\text { Soil } \\
\text { Volume }\left(\mathrm{m}^{3}\right)\end{array}$ & $\begin{array}{l}\text { Total } \\
\text { (pci) }\end{array}$ & $\begin{array}{l}\text { Upper } 95 \div \\
\text { (pCi) }\end{array}$ \\
\hline $5 X-103$ & $0.0-2.5$ & $5 \times 10^{1}$ & 0 & 0 & 0 \\
\hline $5 X-103$ & $0.0-2.5$ & $5 \times 10^{2}$ & 0 & 0 & 0 \\
\hline $5 x-103$ & $0.0-2.5$ & $5 \times 10^{3}$ & 0 & 0 & 0 \\
\hline $5 x-103$ & $0.0-2.5$ & $5 \times 10^{4}$ & 0 & 0 & 0 \\
\hline $5 X-103$ & $0.0-2.5$ & $5 \times 10^{5}$ & 0 & 0 & 0 \\
\hline $5 X-103$ & $0.0-2.5$ & $5 \times 10^{6}$ & 0 & 0 & 0 \\
\hline $5 x-103$ & $0.0-2.5$ & $5 \times 10^{7}$ & 0 & 0 & 0 \\
\hline $5 x-103$ & $0.0-2.5$ & $5 \times 10^{8}$ & 0 & 0 & 0 \\
\hline$S X-103$ & $2.5-5.5$ & $5 \times 10_{2}^{1}$ & 0 & 0 & 0 \\
\hline $5 X-103$ & $2.5-5.5$ & $5 \times 10^{2}$ & 0 & 0 & 0 \\
\hline $5 x-103$ & $2.5-5.5$ & $5 \times 10^{3}$ & 0 & 0 & 0 \\
\hline $5 x-103$ & $2.5-5.5$ & $5 \times 10^{4}$ & 0 & 0 & 0 \\
\hline $5 x-103$ & $2.5-5.5$ & $5 \times 10^{5}$ & 0 & 0 & 0 \\
\hline $5 X-103$ & $2.5-5.5$ & $5 \times 10^{6}$ & 0 & 0 & 0 \\
\hline $5 x-103$ & $2.5-5.5$ & $5 \times 10^{7}$ & 0 & 0 & 0 \\
\hline $5 x-103$ & $2.5-5.5$ & $5 \times 10^{8}$ & 0 & 0 & 0 \\
\hline $5 x-103$ & $5.5-8.5$ & $5 \times 10^{1}$ & 0 & 0 & 0 \\
\hline $5 X-103$ & $5.5-8.5$ & $5 \times 10^{2}$ & 0 & 0 & 0 \\
\hline $5 x-103$ & $5.5-8.5$ & $5 \times 10^{3}$ & 0 & 0 & 0 \\
\hline $5 x-103$ & $5.5-8.5$ & $5 \times 10^{4}$ & 0 & 0 & 0 \\
\hline $5 x-103$ & $5.5-8.5$ & $5 \times 10^{5}$ & 0 & 0 & 0 \\
\hline$s x-103$ & $5.5-8.5$ & $5 \times 10^{6}$ & 0 & 0 & 0 \\
\hline $5 x-103$ & $5.5-8.5$ & $5 \times 10^{7}$ & 0 & 0 & 0 \\
\hline$S X-103$ & $5.5-8.5$ & $5 \times 10^{8}$ & 0 & 0 & 0 \\
\hline $5 x-103$ & $8.5-11.5$ & $5 \times 10^{1}$ & 0 & 0 & 0 \\
\hline $5 x-103$ & $8.5-11.5$ & $5 \times 10^{2}$ & 0 & 0 & 0 \\
\hline$s x-103$ & $8.5-11.5$ & $5 \times 10^{3}$ & 0 & 0 & 0 \\
\hline$s X-103$ & $8.5-11.5$ & $5 \times 10^{4}$ & 0 & 0 & 0 \\
\hline$s x-103$ & $8.5-11.5$ & $5 \times 10^{5}$ & 0 & 0 & 0 \\
\hline$s x-103$ & $8.5-11.5$ & $5 \times 10^{6}$ & 0 & 0 & 0 \\
\hline$s x-103$ & $8.5-11.5$ & $5 \times 10^{7}$ & 0 & 0 & 0 \\
\hline$s x-103$ & $8.5-11.5$ & $5 \times 10^{8}$ & 0 & 0 & 0 \\
\hline$s x-103$ & $11.5-14.5$ & $5 \times 10^{1}$ & 0 & 0 & 0 \\
\hline $5 x-103$ & $11.5-14.5$ & $5 \times 10^{2}$ & 0 & 0 & 0 \\
\hline$S X-103$ & $11.5-14.5$ & $5 \times 10^{3}$ & 0 & 0 & 0 \\
\hline $5 x-103$ & $11.5-14.5$ & $5 \times 10^{4}$ & 0 & 0 & 0 \\
\hline$s x-103$ & $11.5-14.5$ & $5 \times 10^{5}$ & 0 & 0 & 0 \\
\hline$s x-103$ & $11.5-14.5$ & $5 \times 10^{6}$ & 0 & 0 & 0 \\
\hline $5 x-103$ & $11.5-14.5$ & $5 \times 10^{7}$ & 0 & 0 & 0 \\
\hline$s X-103$ & $11.5-14.5$ & $5 \times 10^{8}$ & 0 & 0 & 0 \\
\hline $5 X-103$ & $14.5-17.5$ & $5 \times 10^{1}$ & 0 & 0 & 0 \\
\hline$S X-103$ & $14.5-17.5$ & $5 \times 10^{2}$ & 0 & 0 & 0 \\
\hline$S X-103$ & $14.5-17.5$ & $5 \times 10^{3}$ & 0 & 0 & 0 \\
\hline$s x-103$ & $14.5-17.5$ & $5 \times 10^{4}$ & 0 & 0 & 0 \\
\hline $5 x-103$ & $14.5-17.5$ & $5 \times 10^{5}$ & 0 & 0 & 0 \\
\hline $5 x-103$ & $14.5-17.5$ & $5 \times 10^{6}$ & 0 & 0 & 0 \\
\hline $5 x-103$ & $14.5-17.5$ & $5 \times 10^{7}$ & 0 & 0 & 0 \\
\hline$S X-103$ & $14.5-17.5$ & $5 \times 10^{8}$ & 0 & 0 & 0 \\
\hline $5 x-103$ & $17.5-20.5$ & $5 \times 10^{1}$ & 0 & 0 & 0 \\
\hline $5 x-103$ & $17.5-20.5$ & $5 \times 10^{2}$ & 0 & 0 & 0 \\
\hline$s x-103$ & $17.5-20.5$ & $5 \times 10^{3}$ & 0 & 0 & 0 \\
\hline$S X-103$ & $17.5-20.5$ & $5 \times 10^{4}$ & 0 & 0 & 0 \\
\hline $5 x-103$ & $17.5-20.5$ & $5 \times 10^{5}$ & 0 & 0 & 0 \\
\hline $5 X-103$ & $17.5-20.5$ & $5 \times 10^{6}$ & 0 & 0 & 0 \\
\hline $5 X-103$ & $17.5-20.5$ & $5 \times 10^{7}$ & 0 & 0 & 0 \\
\hline$s x-103$ & $17.5-20.5$ & $5 \times 10^{8}$ & 0 & 0 & 0 \\
\hline $5 x-103$ & $20.5-23.5$ & $5 \times 10^{1}$ & 0 & 0 & 0 \\
\hline$s X-103$ & $20.5-23.5$ & $5 \times 10^{2}$ & 0 & 0 & 0 \\
\hline$s x-103$ & $20.5-23.5$ & $5 \times 10^{3}$ & 0 & 0 & 0 \\
\hline$s x-103$ & $20.5-23.5$ & $5 \times 10^{4}$ & 0 & 0 & 0 \\
\hline
\end{tabular}


Table 4. Continued.

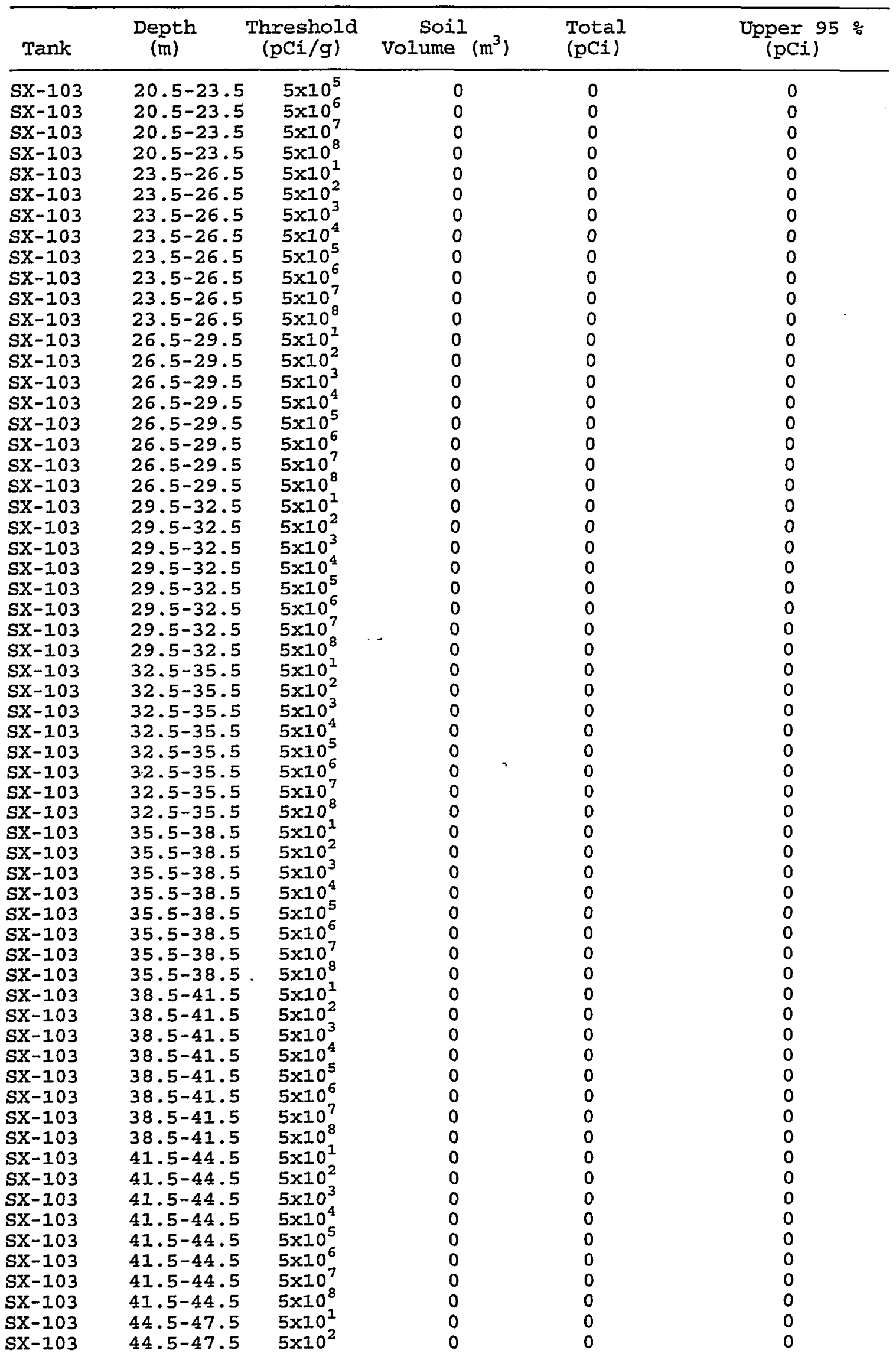


Table 4. Continued.

\begin{tabular}{cccccc}
\hline Tank & $\begin{array}{c}\text { Depth } \\
(\mathrm{m})\end{array}$ & $\begin{array}{c}\text { Threshold } \\
(\mathrm{pCi} / \mathrm{g})\end{array}$ & $\left.\begin{array}{c}\text { Soil } \\
\text { Volume }\end{array} \mathrm{m}^{3}\right)$ & $\begin{array}{c}\text { Total } \\
\text { (pCi) }\end{array}$ & $\begin{array}{c}\text { Upper } 95 \% \\
\text { (pCi) }\end{array}$ \\
\hline $\mathrm{SX}-103$ & $44.5-47.5$ & $5 \times 10^{3}$ & 0 & 0 & 0 \\
$\mathrm{SX}-103$ & $44.5-47.5$ & $5 \times 10^{4}$ & 0 & 0 & 0 \\
$\mathrm{SX}-103$ & $44.5-47.5$ & $5 \times 10^{5}$ & 0 & 0 & 0 \\
$\mathrm{SX}-103$ & $44.5-47.5$ & $5 \times 10^{6}$ & 0 & 0 & 0 \\
$\mathrm{SX}-103$ & $44.5-47.5$ & $5 \times 10^{7}$ & 0 & 0 & 0 \\
$\mathrm{SX}-103$ & $44.5-47.5$ & $5 \times 10^{8}$ & 0 & 0 & 0 \\
$\mathrm{SX}-103$ & $47.5-50.5$ & $5 \times 10^{1}$ & 0 & 0 & 0 \\
$\mathrm{SX}-103$ & $47.5-50.5$ & $5 \times 10^{2}$ & 0 & 0 & 0 \\
$\mathrm{SX}-103$ & $47.5-50.5$ & $5 \times 10^{3}$ & 0 & 0 & 0 \\
$\mathrm{SX}-103$ & $47.5-50.5$ & $5 \times 10^{4}$ & 0 & 0 & 0 \\
$\mathrm{SX}-103$ & $47.5-50.5$ & $5 \times 10^{5}$ & 0 & 0 & 0 \\
$\mathrm{SX}-103$ & $47.5-50.5$ & $5 \times 10^{6}$ & 0 & 0 & 0 \\
$\mathrm{SX}-103$ & $47.5-50.5$ & $5 \times 10^{7}$ & 0 & 0 & 0 \\
$\mathrm{SX}-103$ & $47.5-50.5$ & $5 \times 10^{8}$ & 0 & 0 & 0 \\
\hline
\end{tabular}


Table 5. SX-104 inventory of soil volumes, total activities and upper 95: confidence limit, exceeding 8 activity thresholds over 17 depth increments.

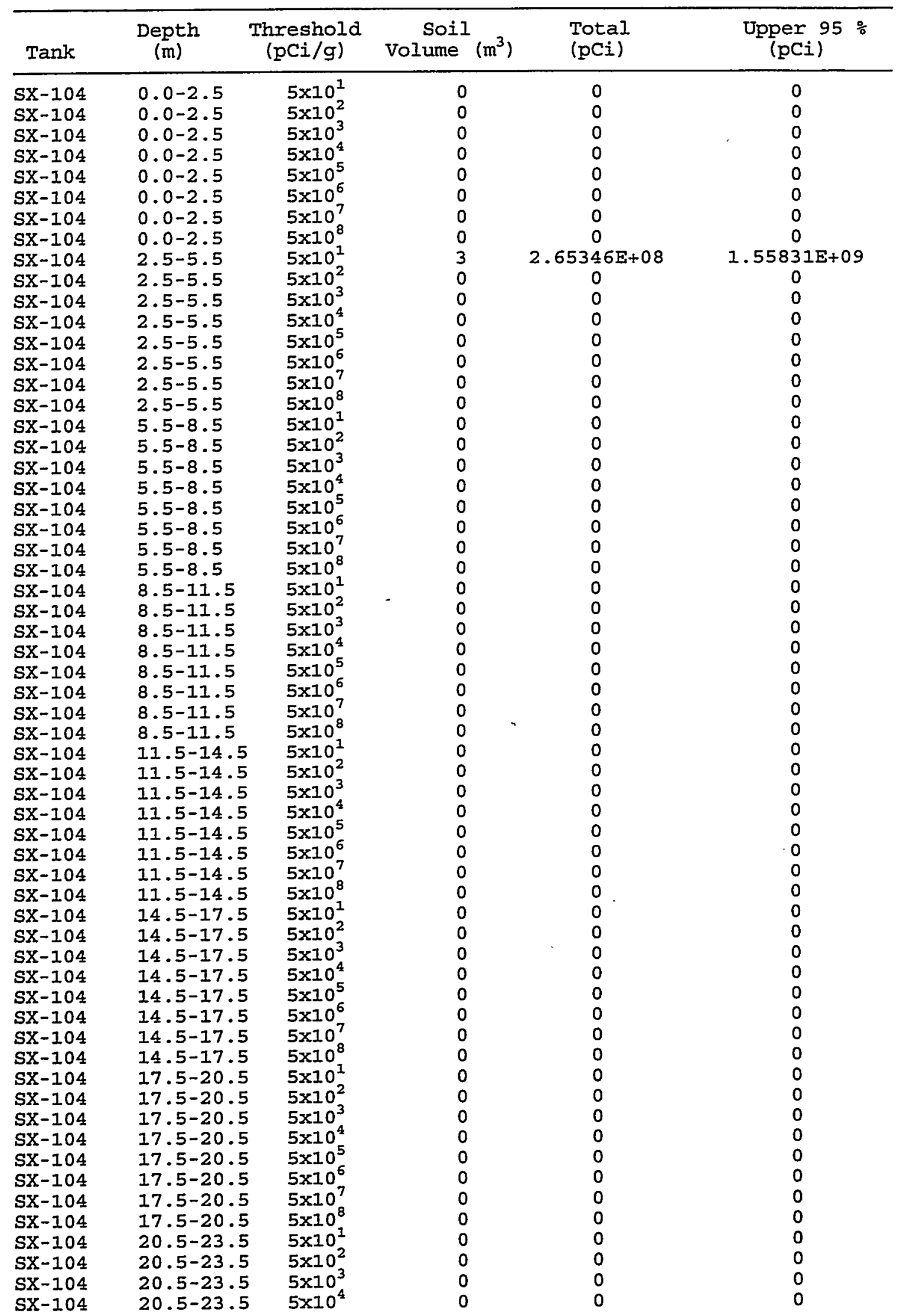


Table 5. Continued.

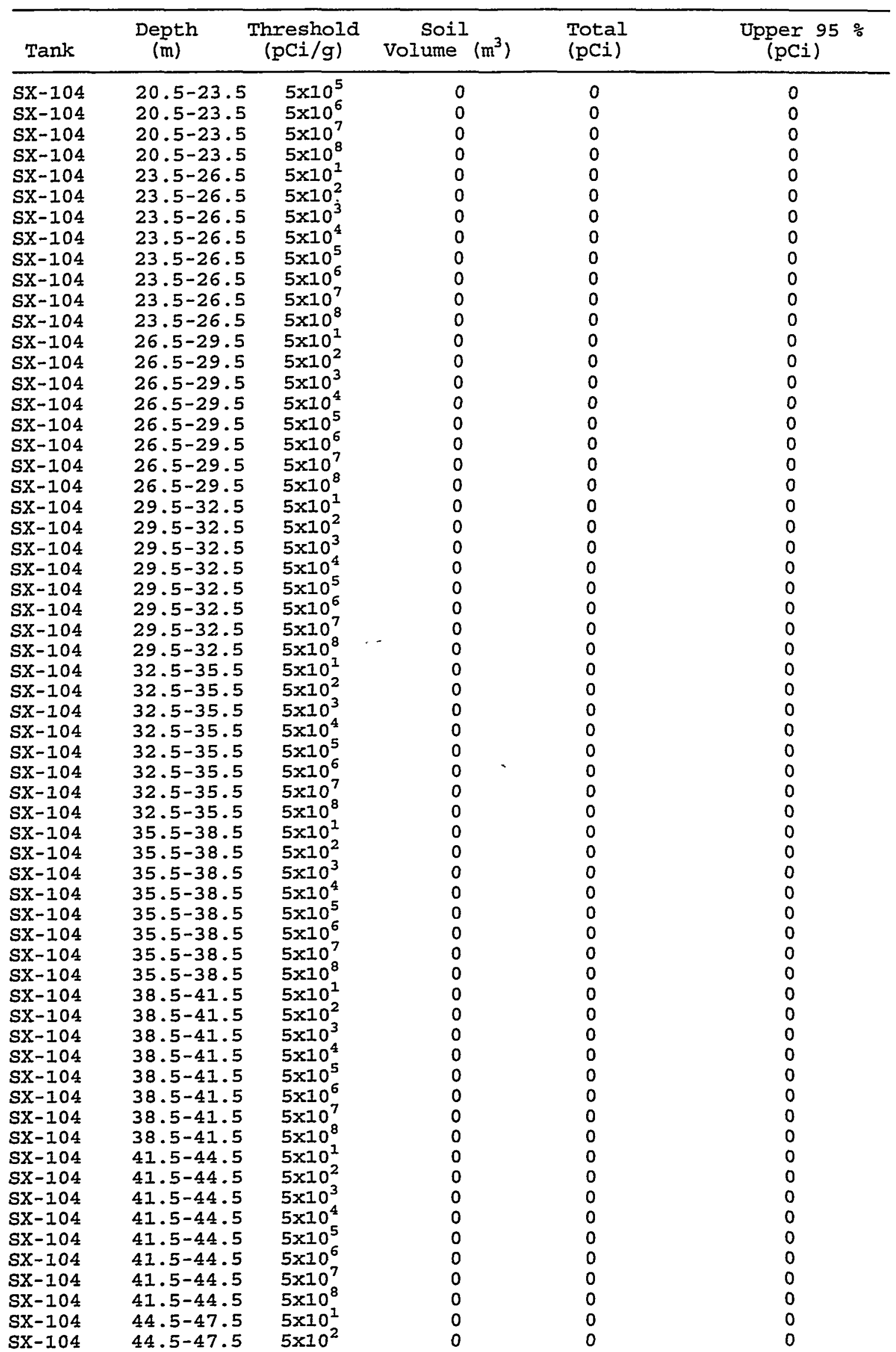


Table 5. Continued.

\begin{tabular}{cccccc}
\hline Tank & $\begin{array}{c}\text { Depth } \\
(\mathrm{m})\end{array}$ & $\begin{array}{c}\text { Threshold } \\
(\mathrm{pCi} / \mathrm{g})\end{array}$ & $\begin{array}{c}\text { Soil } \\
\text { Volume }\end{array}\left(\mathrm{m}^{3}\right)$ & $\begin{array}{c}\text { Total } \\
\text { (pCi) }\end{array}$ & $\begin{array}{c}\text { Upper } 95 \% \\
(\mathrm{pCi})\end{array}$ \\
\hline SX-104 & $44.5-47.5$ & $5 \times 10^{3}$ & 0 & 0 & 0 \\
SX-104 & $44.5-47.5$ & $5 \times 10^{4}$ & 0 & 0 & 0 \\
SX-104 & $44.5-47.5$ & $5 \times 10^{5}$ & 0 & 0 & 0 \\
SX-104 & $44.5-47.5$ & $5 \times 10^{6}$ & 0 & 0 & 0 \\
SX-104 & $44.5-47.5$ & $5 \times 10^{7}$ & 0 & 0 & 0 \\
SX-104 & $44.5-47.5$ & $5 \times 10^{8}$ & 0 & 0 & 0 \\
SX-104 & $47.5-50.5$ & $5 \times 10^{1}$ & 0 & 0 & 0 \\
SX-104 & $47.5-50.5$ & $5 \times 10^{2}$ & 0 & 0 & 0 \\
SX-104 & $47.5-50.5$ & $5 \times 10^{3}$ & 0 & 0 & 0 \\
SX-104 & $47.5-50.5$ & $5 \times 10^{4}$ & 0 & 0 & 0 \\
SX-104 & $47.5-50.5$ & $5 \times 10^{5}$ & 0 & 0 & 0 \\
SX-104 & $47.5-50.5$ & $5 \times 10^{6}$ & 0 & 0 & 0 \\
SX-104 & $47.5-50.5$ & $5 \times 10^{7}$ & 0 & 0 & \\
SX-104 & $47.5-50.5$ & $5 \times 10^{8}$ & 0 & 0 & 0 \\
\hline
\end{tabular}


Table 6. Sx-105 inventory of soil volumes, total activities and upper 95\% confidence limit, exceeding 8 activity thresholds over 17 depth increments.

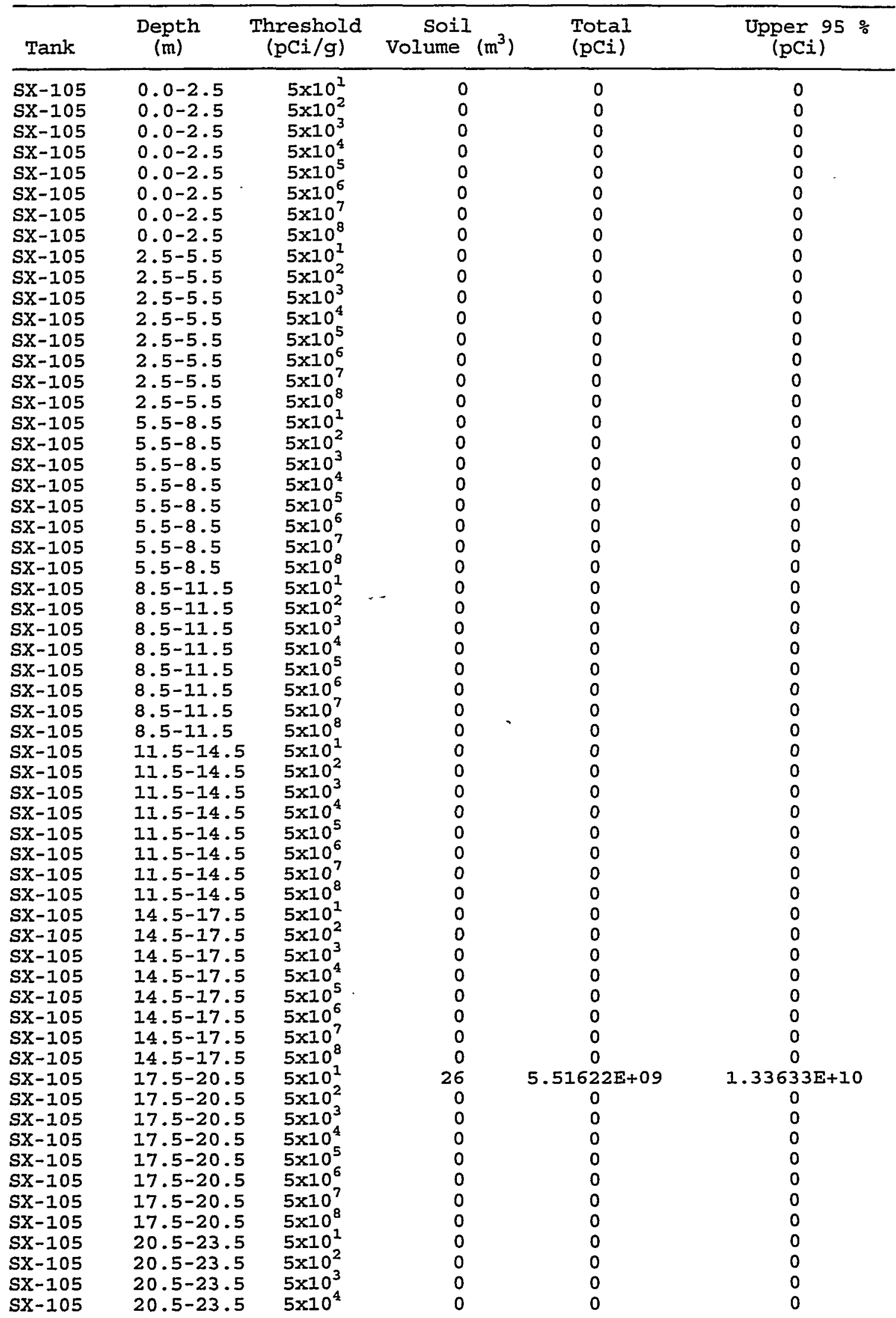


Table 6. Continued.

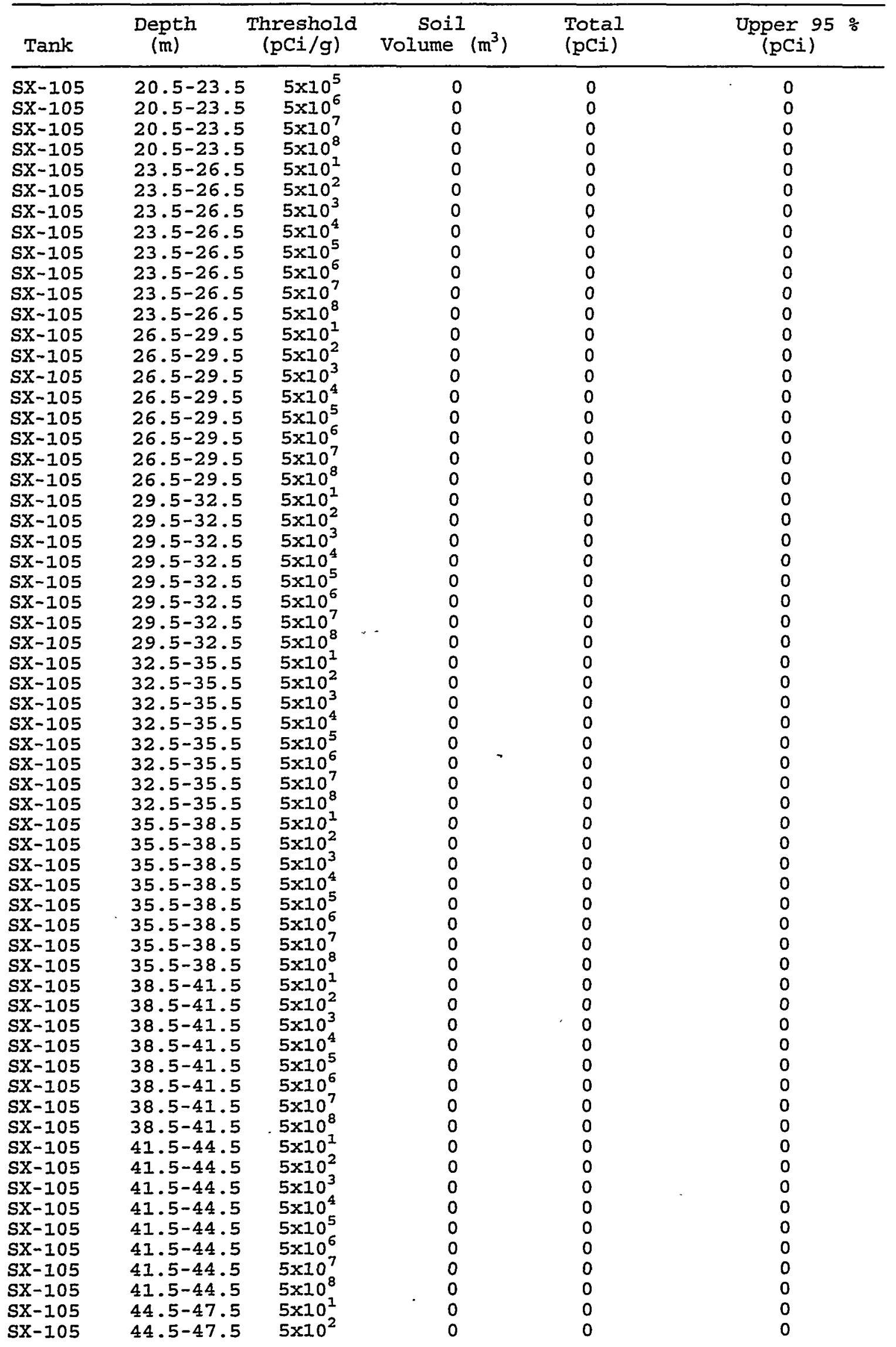


Table 6. Continued.

\begin{tabular}{cccccc}
\hline Tank & $\begin{array}{c}\text { Depth } \\
(\mathrm{m})\end{array}$ & $\begin{array}{c}\text { Threshold } \\
(\mathrm{pCi} / \mathrm{g})\end{array}$ & $\left.\begin{array}{c}\text { Soil } \\
\text { Volume }\end{array} \mathrm{m}^{3}\right)$ & $\begin{array}{c}\text { Total } \\
\text { (pCi) }\end{array}$ & $\begin{array}{c}\text { Upper } 95 \% \\
\text { (pCi) }\end{array}$ \\
\hline SX-105 & $44.5-47.5$ & $5 \times 10^{3}$ & 0 & 0 & 0 \\
SX-105 & $44.5-47.5$ & $5 \times 10^{4}$ & 0 & 0 & 0 \\
SX-105 & $44.5-47.5$ & $5 \times 10^{5}$ & 0 & 0 & 0 \\
SX-105 & $44.5-47.5$ & $5 \times 10^{6}$ & 0 & 0 & 0 \\
SX-105 & $44.5-47.5$ & $5 \times 10^{7}$ & 0 & 0 & 0 \\
SX-105 & $44.5-47.5$ & $5 \times 10^{8}$ & 0 & 0 & 0 \\
SX-105 & $47.5-50.5$ & $5 \times 10^{1}$ & 0 & 0 & 0 \\
SX-105 & $47.5-50.5$ & $5 \times 10^{2}$ & 0 & 0 & 0 \\
SX-105 & $47.5-50.5$ & $5 \times 10^{3}$ & 0 & 0 & 0 \\
SX-105 & $47.5-50.5$ & $5 \times 10^{4}$ & 0 & 0 & 0 \\
SX-105 & $47.5-50.5$ & $5 \times 10^{5}$ & 0 & 0 & 0 \\
SX-105 & $47.5-50.5$ & $5 \times 10^{6}$ & 0 & 0 & 0 \\
SX-105 & $47.5-50.5$ & $5 \times 10^{7}$ & 0 & 0 & 0 \\
SX-105 & $47.5-50.5$ & $5 \times 10^{8}$ & 0 & 0 & 0 \\
\hline
\end{tabular}


Table 7. Sx-106 inventory of soil volumes, total activities and upper 95\% confidence limit, exceeding 8 activity thresholds over 17 depth increments.

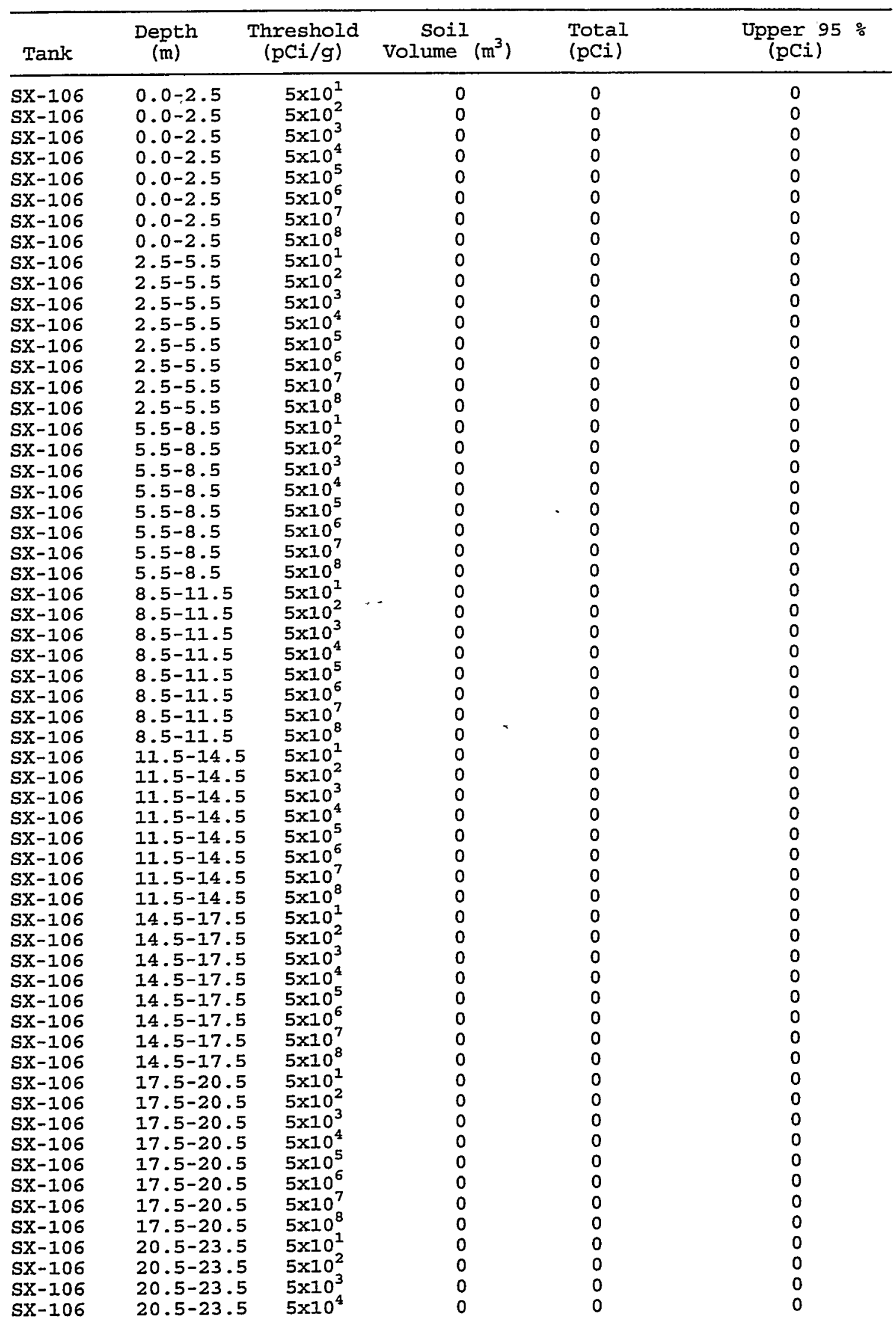


Table 7. Continued.

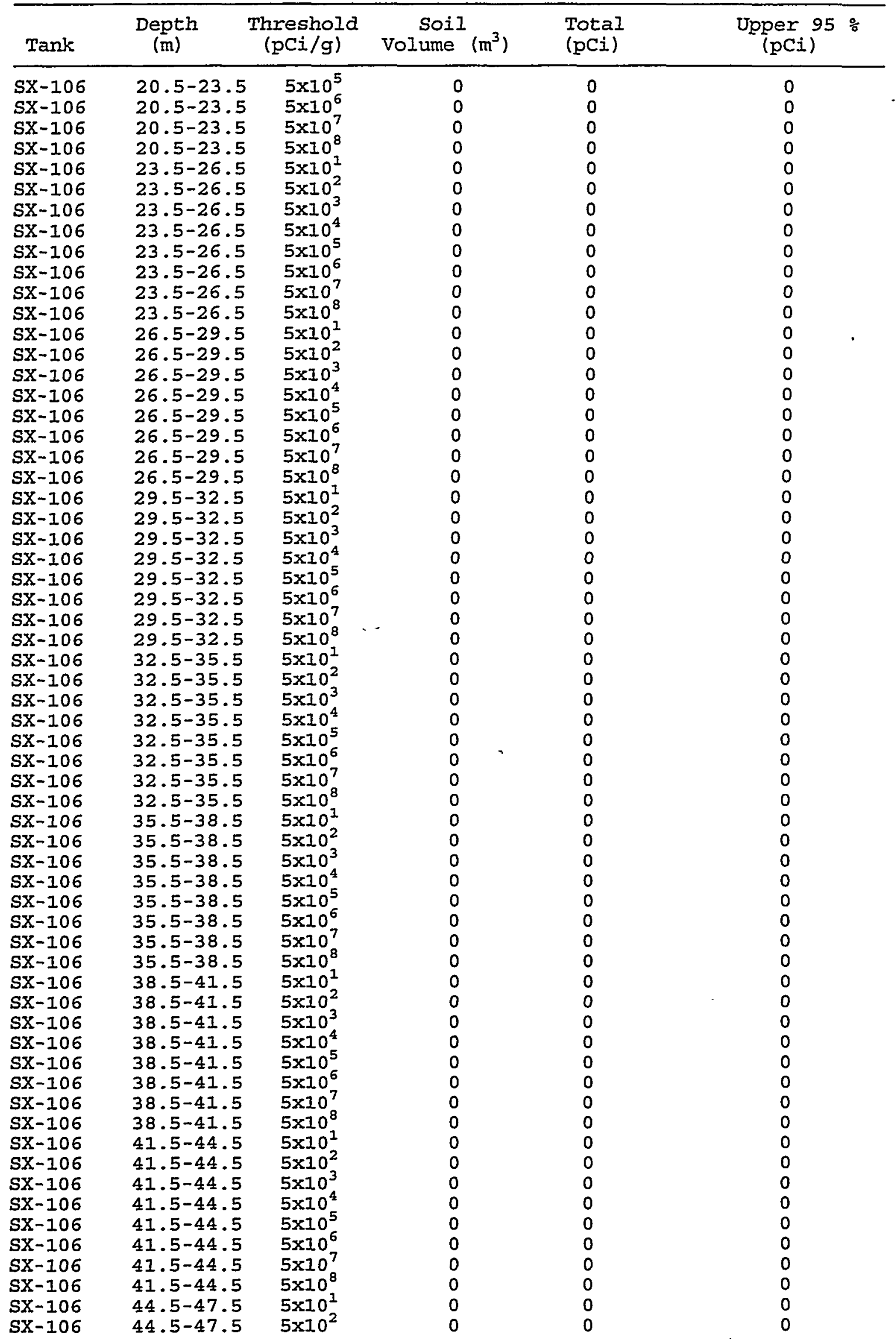


Table 7. Continued.

\begin{tabular}{cccccc}
\hline Tank & $\begin{array}{c}\text { Depth } \\
(\mathrm{m})\end{array}$ & $\begin{array}{c}\text { Threshold } \\
(\mathrm{pCi} / \mathrm{g})\end{array}$ & $\left.\begin{array}{c}\text { Soil } \\
\text { Volume }\end{array} \mathrm{m}^{3}\right)$ & $\begin{array}{c}\text { Total } \\
\text { (pCi) }\end{array}$ & $\begin{array}{c}\text { Upper } 95 \% \\
\text { (pCi) }\end{array}$ \\
\hline SX-106 & $44.5-47.5$ & $5 \times 10^{3}$ & 0 & 0 & 0 \\
SX-106 & $44.5-47.5$ & $5 \times 10^{4}$ & 0 & 0 & 0 \\
SX-106 & $44.5-47.5$ & $5 \times 10^{5}$ & 0 & 0 & 0 \\
SX-106 & $44.5-47.5$ & $5 \times 10^{6}$ & 0 & 0 & 0 \\
SX-106 & $44.5-47.5$ & $5 \times 10^{7}$ & 0 & 0 & 0 \\
SX-106 & $44.5-47.5$ & $5 \times 10^{8}$ & 0 & 0 & 0 \\
SX-106 & $47.5-50.5$ & $5 \times 10^{1}$ & 0 & 0 & 0 \\
SX-106 & $47.5-50.5$ & $5 \times 10^{2}$ & 0 & 0 & 0 \\
SX-106 & $47.5-50.5$ & $5 \times 10^{3}$ & 0 & 0 & 0 \\
SX-106 & $47.5-50.5$ & $5 \times 10^{4}$ & 0 & 0 & 0 \\
SX-106 & $47.5-50.5$ & $5 \times 10^{5}$ & 0 & 0 & 0 \\
SX-106 & $47.5-50.5$ & $5 \times 10^{6}$ & 0 & 0 & 0 \\
SX-106 & $47.5-50.5$ & $5 \times 10^{7}$ & 0 & 0 & 0 \\
SX-106 & $47.5-50.5$ & $5 \times 10^{8}$ & 0 & 0 & 0 \\
\hline
\end{tabular}


Table 8. SX-107 inventory of soil volumes, total activities and upper 95\% confidence limit, exceeding 8 activity thresholds over 17 depth increments.

\begin{tabular}{|c|c|c|c|c|c|}
\hline Tank & $\underset{\text { (m) }}{\text { Depth }}$ & $\begin{array}{l}\text { Threshold } \\
(\mathrm{pCi} / \mathrm{g})\end{array}$ & $\begin{array}{c}\text { Soil } \\
\text { Volume }\left(\mathrm{m}^{3}\right)\end{array}$ & $\begin{array}{l}\text { Total } \\
\text { (pci) }\end{array}$ & $\begin{array}{l}\text { Upper } 95 \% \\
(\text { pCi })\end{array}$ \\
\hline$S X-107$ & $0.0-2.5$ & $5 \times 10^{1}$ & 0 & 0 & 0 \\
\hline $5 x-107$ & $0.0-2.5$ & $5 \times 10^{2}$ & 0 & 0 & 0 \\
\hline$S X-107$ & $0.0-2.5$ & $5 \times 10^{3}$ & 0 & 0 & 0 \\
\hline $5 X-107$ & $0.0-2.5$ & $5 \times 10^{4}$ & 0 & 0 & 0 \\
\hline$s X-107$ & $0.0-2.5$ & $5 \times 10^{5}$ & 0 & 0 & 0 \\
\hline $5 x-107$ & $0.0-2.5$ & $5 \times 10^{6}$ & 0 & 0 & 0 \\
\hline $5 x-107$ & $0.0-2.5$ & $5 \times 10^{7}$ & 0 & 0 & 0 \\
\hline $5 x-107$ & $0.0-2.5$ & $5 \times 10^{8}$ & 0 & 0 & 0 \\
\hline $5 x-107$ & $2.5-5.5$ & $5 \times 10_{2}^{1}$ & 0 & 0 & 0 \\
\hline SX-107 & $2.5-5.5$ & $5 \times 10^{2}$ & 0 & 0 & 0 \\
\hline $5 x-107$ & $2.5-5.5$ & $5 \times 10^{3}$ & 0 & 0 & 0 \\
\hline $5 x-107$ & $2.5-5.5$ & $5 \times 10^{4}$ & 0 & 0 & 0 \\
\hline SX-107 & $2.5-5.5$ & $5 \times 10^{5}$ & 0 & 0 & 0 \\
\hline $5 x-107$ & $2.5-5.5$ & $5 \times 10^{6}$ & 0 & 0 & 0 \\
\hline $5 x-107$ & $2.5-5.5$ & $5 \times 10^{7}$ & 0 & 0 & 0 \\
\hline SX-107 & $2.5-5.5$ & $5 \times 10^{8}$ & 0 & 0 & 0 \\
\hline $5 X-107$ & $5.5-8.5$ & $5 \times 10^{1}$ & 0 & 0 & 0 \\
\hline$s X-107$ & $5.5-8.5$ & $5 \times 10^{2}$ & 0 & 0 & 0 \\
\hline $5 x-107$ & $5.5-8.5$ & $5 \times 10^{3}$ & 0 & 0 & 0 \\
\hline$S X-107$ & $5.5-8.5$ & $5 \times 10^{4}$ & 0 & 0 & 0 \\
\hline $5 X-107$ & $5.5-8.5$ & $5 \times 10^{5}$ & 0 & 0 & 0 \\
\hline$s x-107$ & $5.5-8.5$ & $5 \times 10^{6}$ & 0 & 0 & 0 \\
\hline $5 x-107$ & $5.5-8.5$ & $5 \times 10^{7}$ & 0 & 0 & 0 \\
\hline $5 x-107$ & $5.5-8.5$ & $5 \times 10^{8}$ & 0 & 0 & 0 \\
\hline $5 x-107$ & $8.5-11.5$ & $5 \times 10^{1}$ & 0 & 0 & 0 \\
\hline $5 x-107$ & $8.5-11.5$ & $5 \times 10^{2}$ & 0 & 0 & 0 \\
\hline$s x-107$ & $8.5-11.5$ & $5 \times 10^{3}$ & 0 & 0 & 0 \\
\hline$S x-107$ & $8.5-11.5$ & $5 \times 10^{4}$ & 0 & 0 & 0 \\
\hline$S x-107$ & $8.5-11.5$ & $5 \times 10^{5}$ & 0 & 0 & 0 \\
\hline SX-107 & $8.5-11.5$ & $5 \times 10^{6}$ & 0 & 0 & 0 \\
\hline$S X-107$ & $8.5-11.5$ & $5 \times 10^{7}$ & 0 & 0 & 0 \\
\hline $5 x-107$ & $8.5-11.5$ & $5 \times 10^{8}$ & 0 & 0 & 0 \\
\hline$S X-107$ & $11.5-14.5$ & $5 \times 10^{1}$ & 0 & 0 & 0 \\
\hline$s x-107$ & $11.5-14.5$ & $5 \times 10^{2}$ & 0 & 0 & 0 \\
\hline SX-107 & $11.5-14.5$ & $5 \times 10^{3}$ & 0 & 0 & 0 \\
\hline $5 x-107$ & $11.5-14.5$ & $5 \times 10^{4}$ & 0 & 0 & 0 \\
\hline SX-107 & $11.5-14.5$ & $5 \times 10^{5}$ & 0 & 0 & 0 \\
\hline SX-107 & $11.5-14.5$ & $5 \times 10^{6}$ & 0 & 0 & 0 \\
\hline$S x-107$ & $11.5-14.5$ & $5 \times 10^{7}$ & 0 & 0 & 0 \\
\hline$S X-107$ & $11.5-14.5$ & $5 \times 10^{8}$ & 0 & 0 & 0 \\
\hline $5 x-107$ & $14.5-17.5$ & $5 \times 10^{1}$ & 35 & $8.29995 \mathrm{E}+09$ & $2.13125 \mathrm{E}+10$ \\
\hline $5 x-107$ & $14.5-17.5$ & $5 \times 10^{2}$ & 0 & 0 & 0 \\
\hline$s x-107$ & $14.5-17.5$ & $5 \times 10^{3}$ & 0 & 0 & 0 \\
\hline$S X-107$ & $14.5-17.5$ & $5 \times 10^{4}$ & 0 & 0 & 0 \\
\hline $5 X-107$ & $14.5-17.5$ & $5 \times 10^{5}$ & 0 & 0 & 0 \\
\hline $5 x-107$ & $14.5-17.5$ & $5 \times 10^{6}$ & 0 & 0 & 0 \\
\hline $5 x-107$ & $14.5-17.5$ & $5 \times 10^{7}$ & 0 & 0 & 0 \\
\hline SX-107 & $14.5-17.5$ & $5 \times 10^{8}$ & 0 & 0 & 0 \\
\hline$s x-107$ & $17.5-20.5$ & $5 \times 10^{1}$ & 1160 & $7.51110 E+15$ & $1.35471 \mathrm{E}+16$ \\
\hline SX-107 & $17.5-20.5$ & $5 \times 10^{2}$ & 853 & $7.51101 E+15$ & $1.35469 \mathrm{E}+16$ \\
\hline $5 x-107$ & $17.5-20.5$ & $5 \times 10^{3}$ & 601 & $7.51023 \mathrm{E}+15$ & $1.35455 \mathrm{E}+16$ \\
\hline $5 x-107$ & $17.5-20.5$ & $5 \times 10^{4}$ & 389 & $7.50391 \mathrm{E}+15$ & $1.35322 \mathrm{E}+16$ \\
\hline $5 x-107$ & $17.5-20.5$ & $5 \times 10^{5}$ & 212 & $7.45654 \mathrm{E}+15$ & I. $34306 \mathrm{E}+16$ \\
\hline$s x-107$ & $17.5-20.5$ & $5 \times 10^{6}$ & 87 & $7.07566 \mathrm{E}+15$ & $1.27098 \mathrm{E}+16$ \\
\hline $5 x-107$ & $17.5-20.5$ & $5 \times 10^{7}$ & 24 & $5.42652 \mathrm{E}+15$ & $9.64043 \mathrm{E}+15$ \\
\hline SX-107 & $17.5-20.5$ & $5 \times 10^{8}$ & 0 & 0 & 0 \\
\hline $5 x-107$ & $20.5-23.5$ & $5 \times 10^{1}$ & 946 & $5.15049 \mathrm{E}+14$ & $1.24164 \mathrm{E}+15$ \\
\hline$s X-107$ & $20.5-23.5$ & $5 \times 10^{2}$ & 595 & $5.14941 \mathrm{E}+14$ & $1.24143 \mathrm{E}+15$ \\
\hline$s X-107$ & $20.5-23.5$ & $5 \times 10^{3}$ & 336 & $5.14129 \mathrm{E}+14$ & $1.23956 \mathrm{E}+15$ \\
\hline $5 X-107$ & $20.5-23.5$ & $5 \times 10^{4}$ & 189 & $5.09652 \mathrm{E}+14$ & $1.23022 \mathrm{E}+15$ \\
\hline
\end{tabular}


Table 8. Continued.

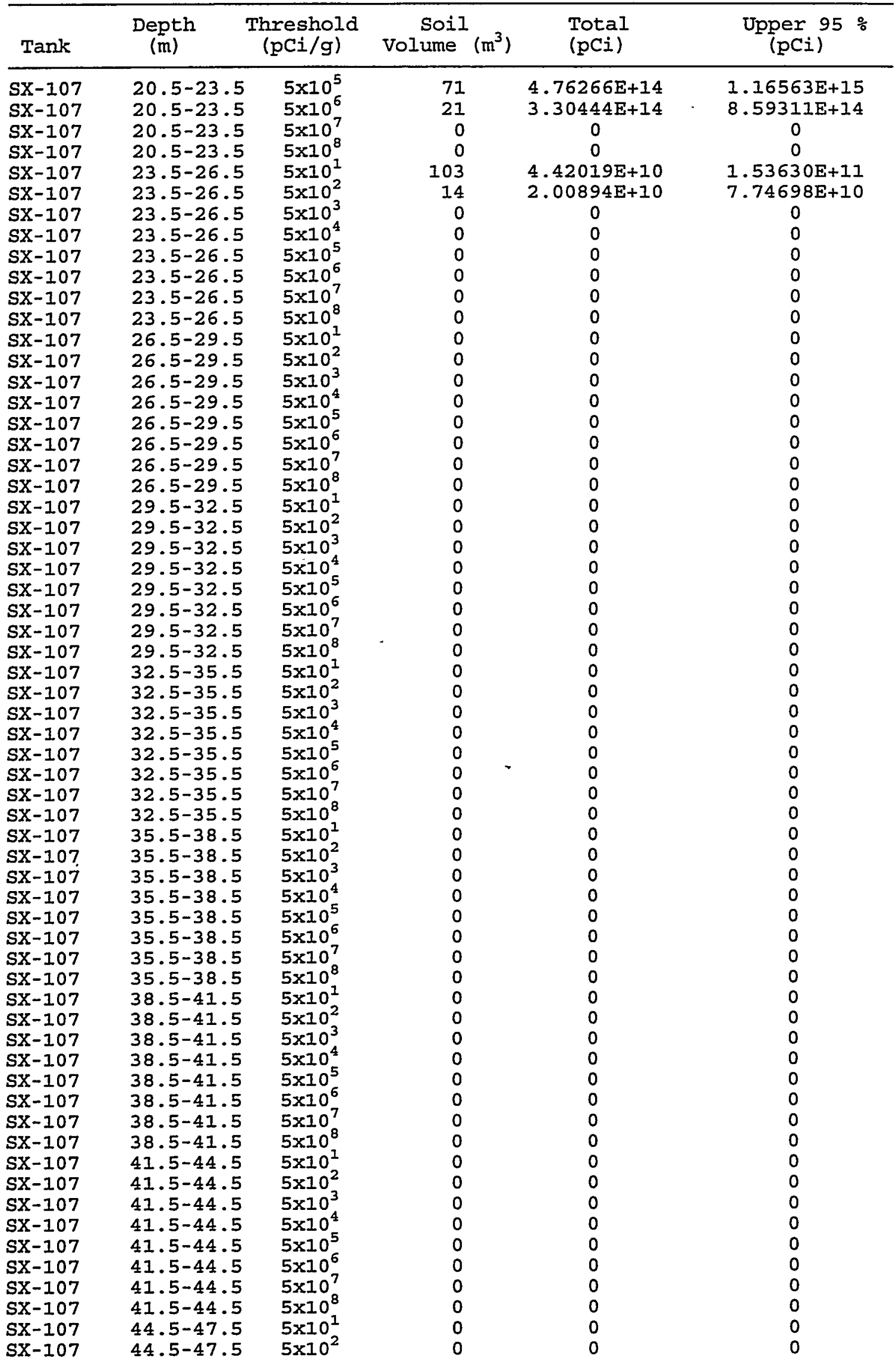


Table 8. Continued.

\begin{tabular}{cccccc}
\hline Tank & $\begin{array}{c}\text { Depth } \\
(\mathrm{m})\end{array}$ & $\begin{array}{c}\text { Threshold } \\
(\mathrm{pCi} / \mathrm{g})\end{array}$ & $\begin{array}{c}\text { Soil } \\
\text { Volume }\end{array}$ & $\begin{array}{c}\text { Total } \\
(\mathrm{pCi})\end{array}$ & $\begin{array}{c}\text { Upper } \\
(\mathrm{pCi})\end{array}$ \\
\hline SX-107 & $44.5-47.5$ & $5 \times 10^{3}$ & 0 & 0 & 0 \\
SX-107 & $44.5-47.5$ & $5 \times 10^{4}$ & 0 & 0 & 0 \\
SX-107 & $44.5-47.5$ & $5 \times 10^{5}$ & 0 & 0 & 0 \\
SX-107 & $44.5-47.5$ & $5 \times 10^{6}$ & 0 & 0 & 0 \\
SX-107 & $44.5-47.5$ & $5 \times 10^{7}$ & 0 & 0 & 0 \\
SX-107 & $44.5-47.5$ & $5 \times 10^{8}$ & 0 & 0 & 0 \\
SX-107 & $47.5-50.5$ & $5 \times 10^{1}$ & 0 & 0 & 0 \\
SX-107 & $47.5-50.5$ & $5 \times 10^{2}$ & 0 & 0 & 0 \\
SX-107 & $47.5-50.5$ & $5 \times 10^{3}$ & 0 & 0 & 0 \\
SX-107 & $47.5-50.5$ & $5 \times 10^{4}$ & 0 & 0 & 0 \\
SX-107 & $47.5-50.5$ & $5 \times 10^{5}$ & 0 & 0 & 0 \\
SX-107 & $47.5-50.5$ & $5 \times 10^{6}$ & 0 & 0 & 0 \\
SX-107 & $47.5-50.5$ & $5 \times 10^{7}$ & 0 & 0 & 0 \\
SX-107 & $47.5-50.5$ & $5 \times 10^{8}$ & 0 & 0 & 0 \\
\hline
\end{tabular}


Table 9. SX-108 inventory of soil volumes, total activities and upper 95\% confidence limit, exceeding 8 activity thresholds over 17 depth increments.

\begin{tabular}{|c|c|c|c|c|c|}
\hline Tank & $\begin{array}{l}\text { Depth } \\
\text { (m) }\end{array}$ & $\begin{array}{c}\text { Threshold } \\
(\mathrm{pCi} / \mathrm{g})\end{array}$ & $\begin{array}{c}\text { Soil } \\
\text { Volume } \\
\left(\mathrm{m}^{3}\right)\end{array}$ & $\begin{array}{l}\text { Total } \\
\text { (pCi) }\end{array}$ & $\begin{array}{l}\text { Upper } 95 \% \\
\text { (pCi) }\end{array}$ \\
\hline SX-108 & $0.0-2.5$ & $5 \times 10^{1}$ & 0 & 0 & 0 \\
\hline $5 x-108$ & $0.0-2.5$ & $5 \times 10^{2}$ & 0 & 0 & 0 \\
\hline $5 X-108$ & $0.0-2.5$ & $5 \times 10^{3}$ & 0 & 0 & 0 \\
\hline$s X-108$ & $0.0-2.5$ & $5 \times 10^{4}$ & 0 & 0 & 0 \\
\hline$s X-108$ & $0.0-2.5$ & $5 \times 10^{5}$ & 0 & 0 & 0 \\
\hline$s X-108$ & $0.0-2.5$ & $5 \times 10^{6}$ & 0 & 0 & 0 \\
\hline$s X-108$ & $0.0-2.5$ & $5 \times 10^{7}$ & 0 & 0 & 0 \\
\hline$s X-108$ & $0.0-2.5$ & $5 \times 10^{8}$ & 0 & 0 & 0 \\
\hline $5 X-108$ & $2.5-5.5$ & $5 \times 10^{1}$ & 0 & 0 & 0 \\
\hline$s X-108$ & $2.5-5.5$ & $5 \times 10^{2}$ & 0 & 0 & 0 \\
\hline$s x-108$ & $2.5-5.5$ & $5 \times 10^{3}$ & 0 & 0 & 0 \\
\hline $5 X-108$ & $2.5-5.5$ & $5 \times 10^{4}$ & 0 & 0 & 0 \\
\hline $5 X-108$ & $2.5-5.5$ & $5 \times 10^{5}$ & 0 & 0 & 0 \\
\hline$s X-108$ & $2.5-5.5$ & $5 \times 10^{6}$ & 0 & 0 & 0 \\
\hline$S X-108$ & $2.5-5.5$ & $5 \times 10^{7}$ & 0 & 0 & 0 \\
\hline $5 x-108$ & $2.5-5.5$ & $5 \times 10^{8}$ & 0 & 0 & 0 \\
\hline $5 X-108$ & $5.5-8.5$ & $5 \times 10^{1}$ & 0 & 0 & 0 \\
\hline $5 X-108$ & $5.5-8.5$ & $5 \times 10^{2}$ & 0 & 0 & 0 \\
\hline $5 x-108$ & $5.5-8.5$ & $5 \times 10^{3}$ & 0 & 0 & 0 \\
\hline$S X-108$ & $5.5-8.5$ & $5 \times 10^{4}$ & 0 & 0 & 0 \\
\hline $5 X-108$ & $5.5-8.5$ & $5 \times 10^{5}$ & 0 & 0 & 0 \\
\hline $5 x-108$ & $5.5-8.5$ & $5 \times 10^{6}$ & 0 & 0 & 0 \\
\hline$s x-108$ & $5.5-8.5$ & $5 \times 10^{7}$ & 0 & 0 & 0 \\
\hline $5 x-108$ & $5.5-8.5$ & $5 \times 10^{8}$ & 0 & 0 & 0 \\
\hline$s x-108$ & $8.5-11.5$ & $5 \times 10^{1}$ & 0 & 0 & 0 \\
\hline$s x-108$ & $8.5-11.5$ & $5 \times 10^{2}$ & 0 & 0 & 0 \\
\hline $5 x-108$ & $8.5-11.5$ & $5 \times 10^{3}$ & 0 & 0 & 0 \\
\hline $5 x-108$ & $8.5-11.5$ & $5 \times 10^{4}$ & 0 & 0 & 0 \\
\hline $5 X-108$ & $8.5-11.5$ & $5 \times 10^{5}$ & 0 & 0 & 0 \\
\hline$s x-108$ & $8.5-11.5$ & $5 \times 10^{6}$ & 0 & 0 & 0 \\
\hline$s X-108$ & $8.5-11.5$ & $5 \times 10^{7}$ & 0 & 0 & 0 \\
\hline $5 X-108$ & $8.5-11.5$ & $5 \times 10^{8}$ & 0 & 0 & 0 \\
\hline $5 x-108$ & $11.5-14.5$ & $5 \times 10^{1}$ & 0 & 0 & 0 \\
\hline $5 x-108$ & $11.5-14.5$ & $5 \times 10_{2}^{2}$ & 0 & 0 & 0 \\
\hline $5 X-108$ & $11.5-14.5$ & $5 \times 10^{3}$ & 0 & 0 & 0 \\
\hline$S X-108$ & $11.5-14.5$ & $5 \times 10^{4}$ & 0 & 0 & 0 \\
\hline$S x-108$ & $11.5-14.5$ & $5 \times 10^{5}$ & 0 & 0 & 0 \\
\hline$s x-108$ & $11.5-14.5$ & $5 \times 10^{6}$ & 0 & 0 & 0 \\
\hline $5 X-108$ & $11.5-14.5$ & $5 \times 10^{7}$ & 0 & 0 & 0 \\
\hline$S X-108$ & $11.5-14.5$ & $5 \times 10^{8}$ & 0 & 0 & 0 \\
\hline$s x-108$ & $14.5-17.5$ & $5 \times 10^{1}$ & 104 & $3.71329 \mathrm{E}+10$ & $1.53973 \mathrm{E}+11$ \\
\hline$s x-108$ & $14.5-17.5$ & $5 \times 10^{2}$ & 13 & $1.66787 \mathrm{E}+10$ & $8.81970 \mathrm{E}+10$ \\
\hline$s x-108$ & $14.5-17.5$ & $5 \times 10^{3}$ & 0 & 0 & 0 \\
\hline$s x-108$ & $14.5-17.5$ & $5 \times 10^{4}$ & 0 & 0 & 0 \\
\hline$s X-108$ & $14.5-17.5$ & $5 \times 10^{5}$ & 0 & 0 & 0 \\
\hline$s x-108$ & $14.5-17.5$ & $5 \times 10^{6}$ & 0 & 0 & 0 \\
\hline$s x-108$ & $14.5-17.5$ & $5 \times 10^{7}$ & 0 & 0 & 0 \\
\hline$S X-108$ & $14.5-17.5$ & $5 \times 10^{8}$ & 0 & 0 & 0 \\
\hline$S x-108$ & $17.5-20.5$ & $5 \times 10^{1}$ & 1912 & $8.46829 \mathrm{E}+15$ & $2.32161 \mathrm{E}+16$ \\
\hline$s x-108$ & $17.5-20.5$ & $5 \times 10^{2}$ & 1499 & $8.46816 \mathrm{E}+15$ & $2.32159 \mathrm{E}+16$ \\
\hline$s x-108$ & $17.5-20.5$ & $5 \times 10^{3}$ & 1116 & $8.46698 \mathrm{E}+15$ & $2.32136 \mathrm{E}+16$ \\
\hline$s x-108$ & $17.5-20.5$ & $5 \times 10^{4}$ & 755 & $8.45547 \mathrm{E}+15$ & $2.31887 \mathrm{E}+16$ \\
\hline$S X-108$ & $17.5-20.5$ & $5 \times 10^{5}$ & 425 & $8.36540 \mathrm{E}+15$ & $2.29652 \mathrm{E}+16$ \\
\hline$S X-108$ & $17.5-20.5$ & $5 \times 10^{6}$ & 200 & $7.59480 \mathrm{E}+15$ & $2.09169 \mathrm{E}+16$ \\
\hline $5 x-108$ & $17.5-20.5$ & $5 \times 10^{7}$ & 0 & 0 & 0 \\
\hline$S X-108$ & $17.5-20.5$ & $5 \times 10^{8}$ & 0 & 0 & 0 \\
\hline$s x-108$ & $20.5-23.5$ & $5 \times 10^{1}$ & 1794 & $5.81604 \mathrm{E}+15$ & $1.29206 \mathrm{E}+16$ \\
\hline$s X-108$ & $20.5-23.5$ & $5 \times 10^{2}$ & 1473 & $5.81594 E+15$ & $1.29204 \mathrm{E}+16$ \\
\hline$s x-108$ & $20.5-23.5$ & $5 \times 10^{3}$ & 1142 & $5.81497 \mathrm{E}+15$ & $1.29184 \mathrm{E}+16$ \\
\hline$s x-108$ & $20.5-23.5$ & $5 \times 10^{4}$ & 789 & $5.80350 \mathrm{E}+15$ & $1.28943 \mathrm{E}+16$ \\
\hline
\end{tabular}


HNF-5782 Rev.0

Table 9. Continued.

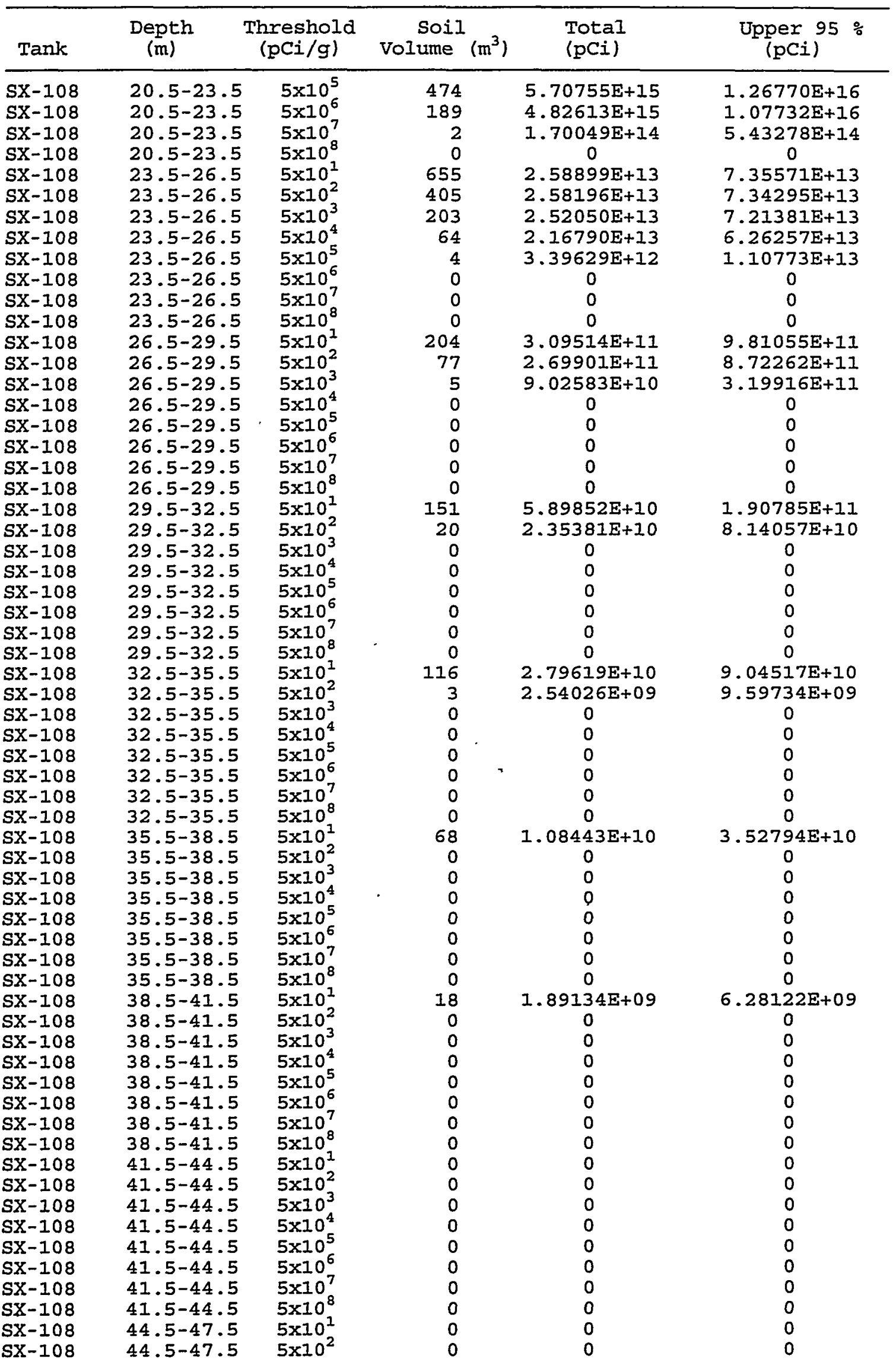


Table 9. Continued.

\begin{tabular}{cccccc}
\hline Tank & $\begin{array}{c}\text { Depth } \\
(\mathrm{m})\end{array}$ & $\begin{array}{c}\text { Threshold } \\
(\mathrm{pCi} / \mathrm{g})\end{array}$ & $\begin{array}{c}\text { Soil } \\
\text { Volume }\left(\mathrm{m}^{3}\right)\end{array}$ & $\begin{array}{c}\text { Total } \\
(\mathrm{pCi})\end{array}$ & $\begin{array}{c}\text { Upper 95 } \\
(\mathrm{pCi})\end{array}$ \\
\hline SX-108 & $44.5-47.5$ & $5 \times 10^{3}$ & 0 & 0 & 0 \\
SX-108 & $44.5-47.5$ & $5 \times 10^{4}$ & 0 & 0 & 0 \\
SX-108 & $44.5-47.5$ & $5 \times 10^{5}$ & 0 & 0 & 0 \\
SX-108 & $44.5-47.5$ & $5 \times 10^{6}$ & 0 & 0 & 0 \\
SX-108 & $44.5-47.5$ & $5 \times 10^{7}$ & 0 & 0 & 0 \\
SX-108 & $44.5-47.5$ & $5 \times 10^{8}$ & 0 & 0 & 0 \\
SX-108 & $47.5-50.5$ & $5 \times 10^{1}$ & 0 & 0 & 0 \\
SX-108 & $47.5-50.5$ & $5 \times 10^{2}$ & 0 & 0 & 0 \\
SX-108 & $47.5-50.5$ & $5 \times 10^{3}$ & 0 & 0 & 0 \\
SX-108 & $47.5-50.5$ & $5 \times 10^{4}$ & 0 & 0 & 0 \\
SX-108 & $47.5-50.5$ & $5 \times 10^{5}$ & 0 & 0 & 0 \\
SX-108 & $47.5-50.5$ & $5 \times 10^{6}$ & 0 & 0 & 0 \\
SX-108 & $47.5-50.5$ & $5 \times 10^{7}$ & 0 & 0 & 0 \\
SX-108 & $47.5-50.5$ & $5 \times 10^{8}$ & 0 & 0 & 0 \\
\hline
\end{tabular}


Table 10. SX-109 inventory of soil volumes, total activities and upper 95\% confidence limit, exceeding 8 activity thresholds over 17 depth increments.

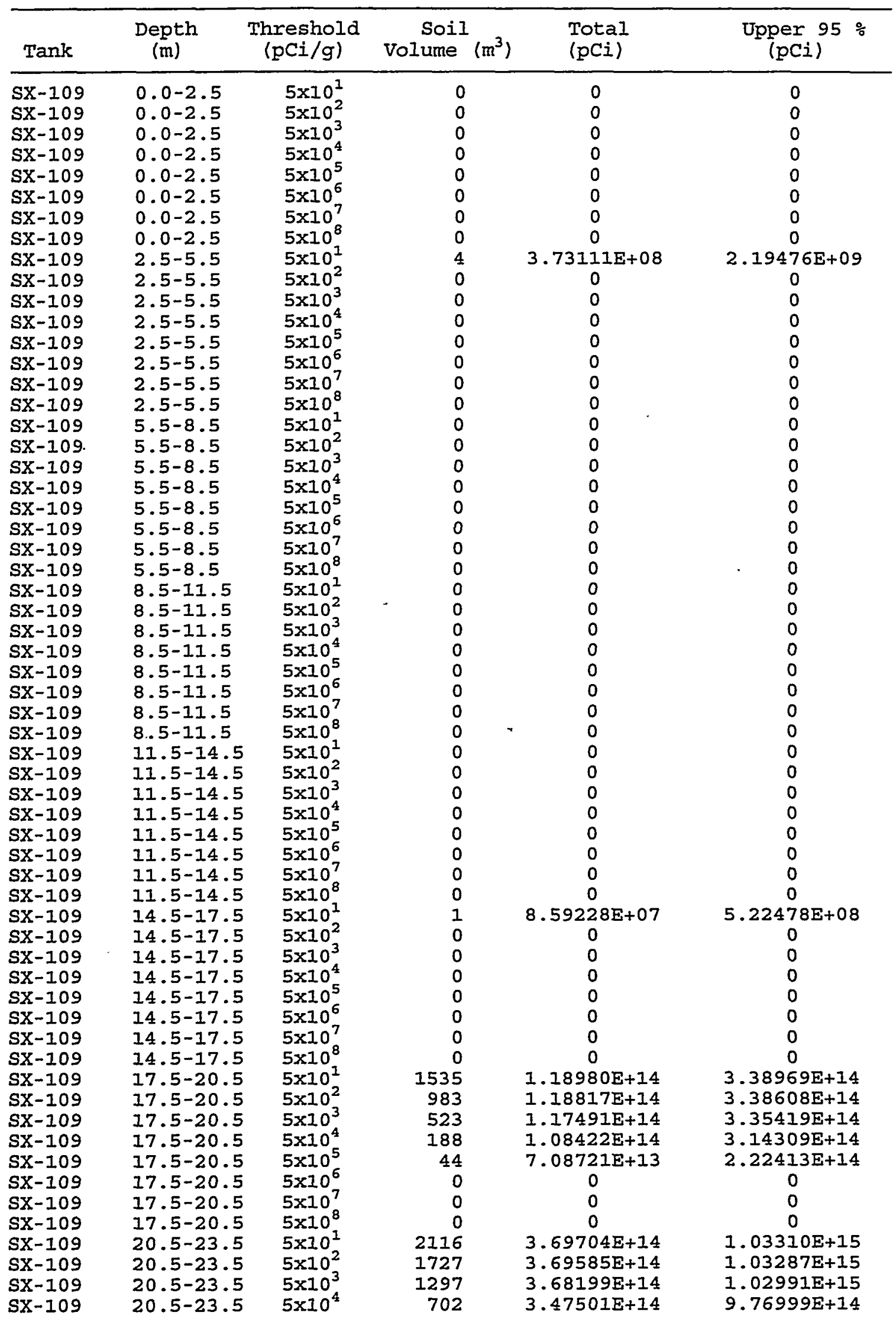


Table 10. Continued.

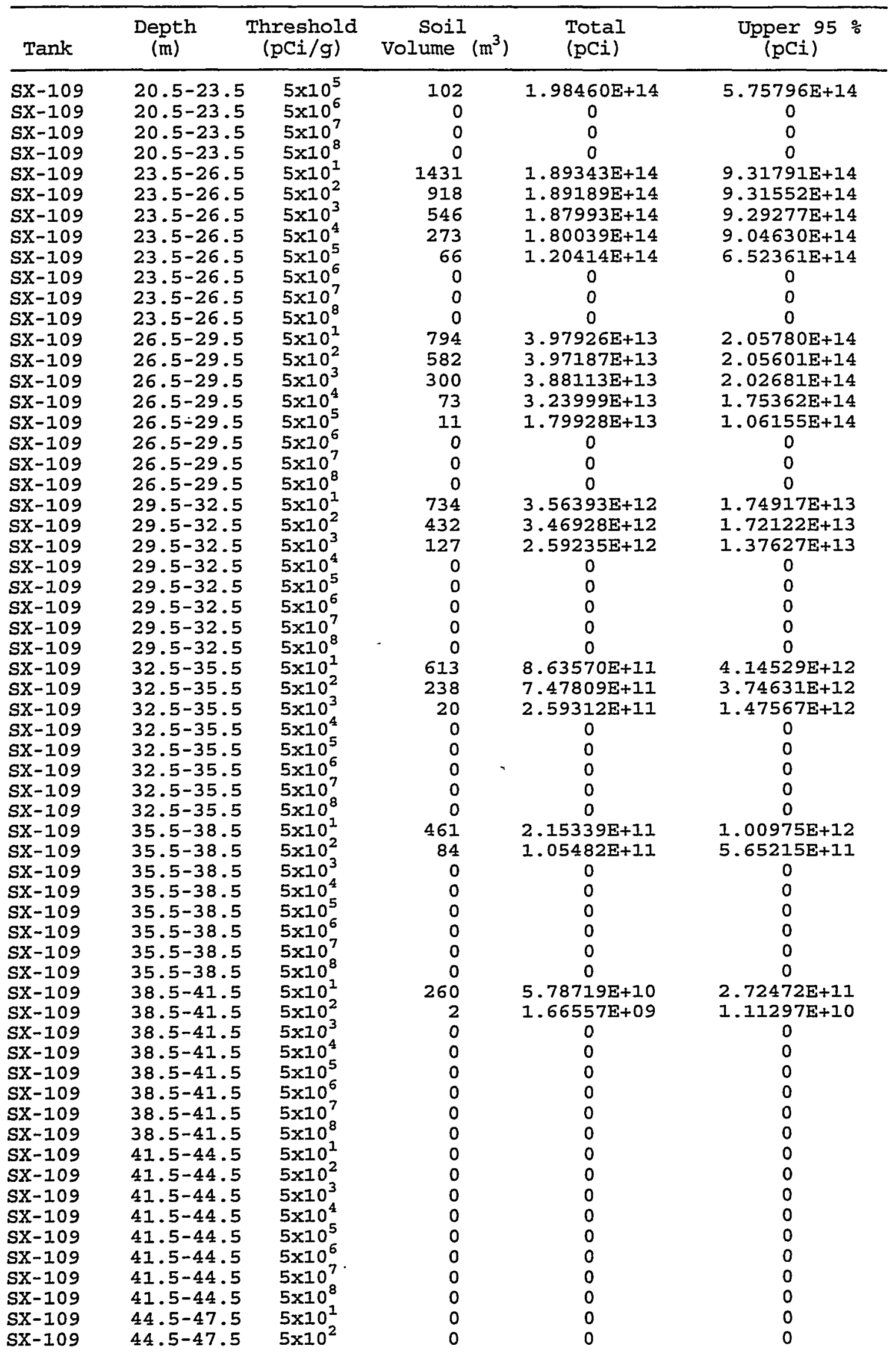


HNF-5782 Rev.0

Table 10. Continued.

\begin{tabular}{|c|c|c|c|c|c|}
\hline Tank & $\underset{(m)}{\text { Depth }}$ & $\begin{array}{l}\text { Threshold } \\
\text { (pCi/g) }\end{array}$ & $\begin{array}{l}\text { Soil } \\
\text { Volume }\left(\mathrm{m}^{3}\right)\end{array}$ & $\begin{array}{l}\text { Total } \\
\text { (pCi) }\end{array}$ & $\begin{array}{l}\text { Upper } \\
\text { (pCi) }\end{array}$ \\
\hline sX-109 & $44.5-47.5$ & $5 \times 10^{3}$ & 0 & 0 & 0 \\
\hline SX-109 & $44.5-47.5$ & $5 \times 10^{4}$ & 0 & 0 & 0 \\
\hline $5 x-109$ & $44.5-47.5$ & $5 \times 10^{5}$ & 0 & 0 & 0 \\
\hline$s x-109$ & $44.5-47.5$ & $5 \times 10^{6}$ & 0 & 0 & 0 \\
\hline SX-109 & $44.5-47.5$ & $5 \times 10^{7}$ & 0 & 0 & 0 \\
\hline SX-109 & $44.5-47.5$ & $5 \times 10^{8}$ & 0 & 0 & 0 \\
\hline Sx-109 & $47.5-50.5$ & $5 \times 10^{1}$ & 0 & 0 & 0 \\
\hline $5 x-109$ & $47.5-50.5$ & $5 \times 10^{2}$ & 0 & 0 & 0 \\
\hline Sx-109 & $47.5-50.5$ & $5 \times 10^{3}$ & 0 & 0 & 0 \\
\hline sx-109 & $47.5-50.5$ & $5 \times 10^{4}$ & 0 & 0 & 0 \\
\hline $5 x-109$ & $47.5-50.5$ & $5 \times 10^{5}$ & 0 & 0 & 0 \\
\hline Sx-109 & $47.5-50.5$ & $5 \times 10^{6}$ & 0 & 0 & 0 \\
\hline $5 x-109$ & $47.5-50.5$ & $5 \times 10^{7}$ & 0 & 0 & 0 \\
\hline $5 x-109$ & $47.5-50.5$ & $5 \times 10^{8}$ & 0 & 0 & 0 \\
\hline
\end{tabular}


Table 11. sX-110 inventory of soil volumes, total activities and upper 95\% confidence limit, exceeding 8 activity thresholds over 17 depth increments.

\begin{tabular}{|c|c|c|c|c|c|}
\hline Tank & $\underset{(m)}{\text { Depth }}$ & $\begin{array}{l}\text { Threshold } \\
\text { (pCi/g) }\end{array}$ & $\begin{array}{l}\text { Soil } \\
\text { Volume }\left(\mathrm{m}^{3}\right)\end{array}$ & $\begin{array}{l}\text { Total } \\
\text { (pCi) }\end{array}$ & $\begin{array}{l}\text { Upper } 95 \div \\
\text { (pCi) }\end{array}$ \\
\hline$s X-110$ & $0.0-2.5$ & $5 \times 10^{1}$ & 0 & 0 & 0 \\
\hline $5 x-110$ & $0.0-2.5$ & $5 \times 10^{2}$ & 0 & 0 & 0 \\
\hline$s x-110$ & $0.0-2.5$ & $5 \times 10^{3}$ & 0 & 0 & 0 \\
\hline$s x-110$ & $0.0-2.5$ & $5 \times 10^{4}$ & 0 & 0 & 0 \\
\hline$s x-110$ & $0.0-2.5$ & $5 \times 10^{5}$ & 0 & 0 & 0 \\
\hline$s x-110$ & $0.0-2.5$ & $5 \times 10^{6}$ & 0 & 0 & 0 \\
\hline$s x-110$ & $0.0-2.5$ & $5 \times 10^{7}$ & 0 & 0 & 0 \\
\hline$s x-110$ & $0.0-2.5$ & $5 \times 10^{8}$ & 0 & 0 & 0 \\
\hline $5 x-110$ & $2.5-5.5$ & $5 \times 10^{1}$ & 0 & 0 & 0 \\
\hline $5 x-110$ & $2.5-5.5$ & $5 \times 10^{2}$ & 0 & 0 & 0 \\
\hline $5 x-110$ & $2.5-5.5$ & $5 \times 10^{3}$ & 0 & 0 & 0 \\
\hline$s X-110$ & $2.5-5.5$ & $5 \times 10^{4}$ & 0 & 0 & 0 \\
\hline $5 x-110$ & $2.5-5.5$ & $5 \times 10^{5}$ & 0 & 0 & 0 \\
\hline $5 X-110$ & $2.5-5.5$ & $5 \times 10^{6}$ & 0 & 0 & 0 \\
\hline$s x-110$ & $2.5-5.5$ & $5 \times 10^{7}$ & 0 & 0 & 0 \\
\hline $5 X-110$ & $2.5-5.5$ & $5 \times 10^{8}$ & 0 & 0 & 0 \\
\hline$s x-110$ & $5.5-8.5$ & $5 \times 10^{1}$ & 0 & 0 & 0 \\
\hline $5 x-110$ & $5.5-8.5$ & $5 \times 10^{2}$ & 0 & 0 & 0 \\
\hline $5 x-110$ & $5.5-8.5$ & $5 \times 10^{3}$ & 0 & 0 & 0 \\
\hline$s X-110$ & $5.5-8.5$ & $5 \times 10^{4}$ & 0 & 0 & 0 \\
\hline $5 X-110$ & $5.5-8.5$ & $5 \times 10^{5}$ & 0 & 0 & 0 \\
\hline $5 x-110$ & $5.5-8.5$ & $5 \times 10^{6}$ & 0 & 0 & 0 \\
\hline$S X-110$ & $5.5-8.5$ & $5 \times 10^{7}$ & 0 & 0 & 0 \\
\hline$S X-110$ & $5.5-8.5$ & $5 \times 10^{8}$ & 0 & 0 & 0 \\
\hline$s X-110$ & $8.5-11.5$ & $5 \times 10^{1}$ & 0 & 0 & 0 \\
\hline$S X-110$ & $8.5-11.5$ & $5 \times 10^{2}$ & 0 & 0 & 0 \\
\hline $5 x-110$ & $8.5-11.5$ & $5 \times 10^{3}$ & 0 & 0 & 0 \\
\hline$s x-110$ & $8.5-11.5$ & $5 \times 10^{4}$ & 0 & 0 & 0 \\
\hline$s x-110$ & $8.5-11.5$ & $5 \times 10^{5}$ & 0 & 0 & 0 \\
\hline$s x-110$ & $8.5-11.5$ & $5 \times 10^{6}$ & 0 & 0 & 0 \\
\hline$s x-110$ & $8.5-11.5$ & $5 \times 10^{7}$ & 0 & 0 & 0 \\
\hline$s x-110$ & $8.5-11.5$ & $5 \times 10^{8}$ & 0 & 0 & 0 \\
\hline $5 x-110$ & $11.5-14.5$ & $5 \times 10^{1}$ & 0 & 0 & 0 \\
\hline$S X-110$ & $11.5-14.5$ & $5 \times 10^{2}$ & 0 & 0 & 0 \\
\hline$S X-110$ & $11.5-14.5$ & $5 \times 10^{3}$ & 0 & 0 & 0 \\
\hline$S X-110$ & $11.5-14.5$ & $5 \times 10^{4}$ & 0 & 0 & 0 \\
\hline$S X-110$ & $11.5-14.5$ & $5 \times 10^{5}$ & 0 & 0 & 0 \\
\hline$s x-110$ & $11.5-14.5$ & $5 \times 10^{6}$ & 0 & 0 & 0 \\
\hline$s x-110$ & $11.5-14.5$ & $5 \times 10^{7}$ & 0 & 0 & 0 \\
\hline$s x-110$ & $11.5-14.5$ & $5 \times 10^{8}$ & 0 & 0 & 0 \\
\hline$s x-110$ & $14.5-17.5$ & $5 \times 10^{1}$ & 0 & 0 & 0 \\
\hline$s x-110$ & $14.5-17.5$ & $5 \times 10^{2}$ & 0 & 0 & 0 \\
\hline$s X-110$ & $14.5-17.5$ & $5 \times 10^{3}$ & 0 & 0 & 0 \\
\hline$s x-110$ & $14.5-17.5$ & $5 \times 10^{4}$ & 0 & 0 & 0 \\
\hline $5 x-110$ & $14.5-17.5$ & $5 \times 10^{5}$ & 0 & 0 & 0 \\
\hline$S x-110$ & $14.5-17.5$ & $5 \times 10^{6}$ & 0 & 0 & 0 \\
\hline$s x-110$ & $14.5-17.5$ & $5 \times 10^{7}$ & 0 & 0 & 0 \\
\hline$s x-110$ & $14.5-17.5$ & $5 \times 10^{8}$ & 0 & 0 & 0 \\
\hline$S X-110$ & $17.5-20.5$ & $5 \times 10^{1}$ & 40 & $1.87009 \mathrm{E}+12$ & $1.20144 \mathrm{E}+13$ \\
\hline$s x-110$ & $17.5-20.5$ & $5 \times 10^{2}$ & 19 & $1.86414 E+12$ & $1.19872 \mathrm{E}+13$ \\
\hline$s x-110$ & $17.5-20.5$ & $5 \times 10^{3}$ & 7 & $1.82641 \mathrm{E}+12$ & $1.17984 \mathrm{E}+13$ \\
\hline$s x-110$ & $17.5-20.5$ & $5 \times 10^{4}$ & 4 & $1.76547 \mathrm{E}+12$ & $1.14944 \mathrm{E}+13$ \\
\hline$s x-110$ & $17.5-20.5$ & $5 \times 10^{5}$ & 1 & $8.92941 E+11$ & $6.59409 \mathrm{E}+12$ \\
\hline$s x-110$ & $17.5-20.5$ & $5 \times 10^{6}$ & 0 & 0 & 0 \\
\hline $5 x-110$ & $17.5-20.5$ & $5 \times 10^{7}$ & 0 & 0 & 0 \\
\hline $5 x-110$ & $17.5-20.5$ & $5 \times 10^{8}$ & 0 & 0 & 0 \\
\hline $5 x-110$ & $20.5-23.5$ & $5 \times 10^{1}$ & 26 & $1.05946 \mathrm{E}+11$ & $6.16090 \mathrm{E}+11$ \\
\hline $5 x-110$ & $20.5-23.5$ & $5 \times 10^{2}$ & 10 & $1.01823 \mathrm{E}+11$ & $5.96824 \mathrm{E}+11$ \\
\hline $5 x-110$ & $20.5-23.5$ & $5 \times 10^{3}$ & 4 & $8.51733 \mathrm{E}+10$ & $5.13605 \mathrm{E}+11$ \\
\hline$S X-110$ & $20.5-23.5$ & $5 \times 10^{4}$ & 0 & 0 & 0 \\
\hline
\end{tabular}


Table 11. Continued.

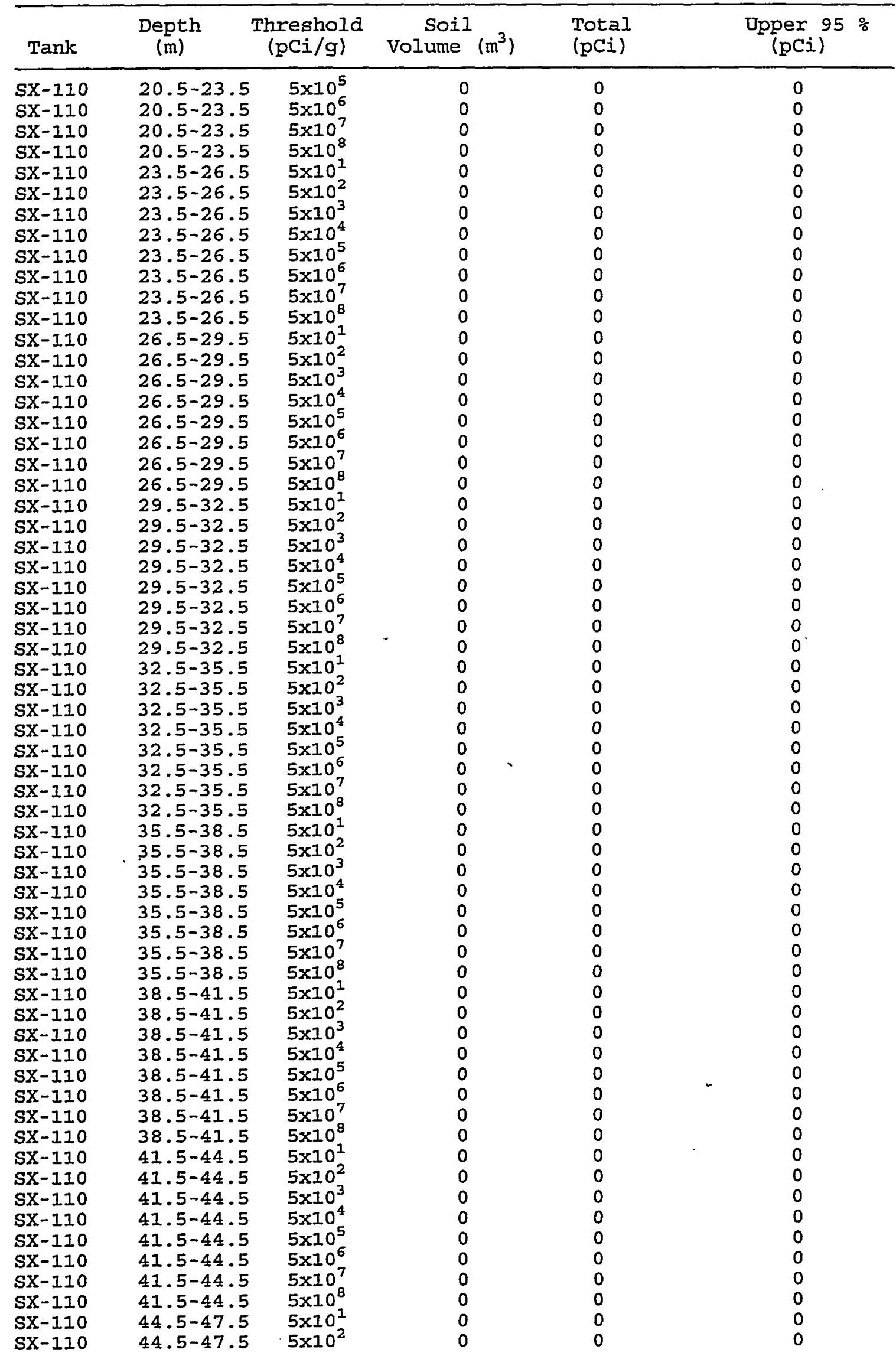


HNF-5782 Rev. 0

Table 11. Continued.

\begin{tabular}{cccccc}
\hline Tank & $\begin{array}{c}\text { Depth } \\
(\mathrm{m})\end{array}$ & $\begin{array}{c}\text { Threshold } \\
(\mathrm{pCi} / \mathrm{g})\end{array}$ & $\left.\begin{array}{c}\text { Soil } \\
\text { Volume }\end{array} \mathrm{m}^{3}\right)$ & $\begin{array}{c}\text { Total } \\
(\mathrm{pCi})\end{array}$ & $\begin{array}{c}\text { Upper } 95 \% \\
(\mathrm{pC} i)^{\circ}\end{array}$ \\
\hline SX-110 & $44.5-47.5$ & $5 \times 10^{3}$ & 0 & 0 & 0 \\
SX-110 & $44.5-47.5$ & $5 \times 10^{4}$ & 0 & 0 & 0 \\
SX-110 & $44.5-47.5$ & $5 \times 10^{5}$ & 0 & 0 & 0 \\
SX-110 & $44.5-47.5$ & $5 \times 10^{6}$ & 0 & 0 & 0 \\
SX-110 & $44.5-47.5$ & $5 \times 10^{7}$ & 0 & 0 & 0 \\
SX-110 & $44.5-47.5$ & $5 \times 10^{8}$ & 0 & 0 & 0 \\
SX-110 & $47.5-50.5$ & $5 \times 10^{1}$ & 0 & 0 & 0 \\
SX-110 & $47.5-50.5$ & $5 \times 10^{2}$ & 0 & 0 & 0 \\
SX-110 & $47.5-50.5$ & $5 \times 10^{3}$ & 0 & 0 & 0 \\
SX-110 & $47.5-50.5$ & $5 \times 10^{4}$ & 0 & 0 & 0 \\
SX-110 & $47.5-50.5$ & $5 \times 10^{5}$ & 0 & 0 & 0 \\
SX-110 & $47.5-50.5$ & $5 \times 10^{6}$ & 0 & 0 & 0 \\
SX-110 & $47.5-50.5$ & $5 \times 10^{7}$ & 0 & 0 & 0 \\
SX-110 & $47.5-50.5$ & $5 \times 10^{8}$ & 0 & 0 & 0 \\
\hline
\end{tabular}


Table 12. SX-1II inventory of soil volumes, total activities and upper 95\% confidence limit, exceeding 8 activity thresholds over 17 depth increments.

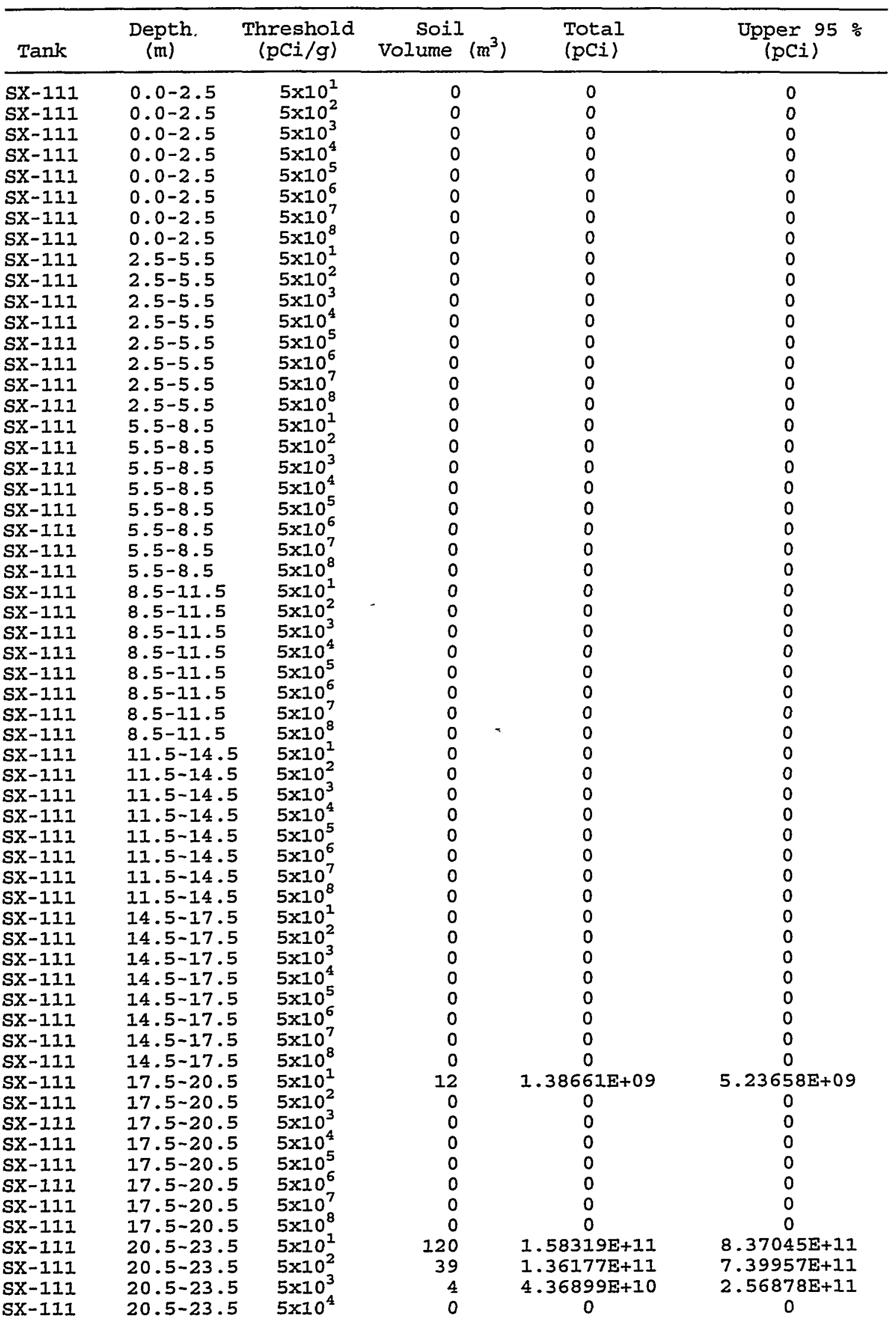


Table 12. Continued.




HNF-5782 Rev.0

Table 12. Continued.

\begin{tabular}{cccccc}
\hline Tank & $\begin{array}{c}\text { Depth } \\
(\mathrm{m})\end{array}$ & $\begin{array}{c}\text { Threshold } \\
(\mathrm{pCi} / \mathrm{g})\end{array}$ & $\begin{array}{c}\text { Soil } \\
\text { Volume } \\
\left(\mathrm{m}^{3}\right)\end{array}$ & $\begin{array}{c}\text { Total } \\
\text { (pCi) }\end{array}$ & $\begin{array}{c}\text { Upper 95 } \\
(\mathrm{pCi})\end{array}$ \\
\hline SX-111 & $44.5-47.5$ & $5 \times 10^{3}$ & 0 & 0 & 0 \\
SX-111 & $44.5-47.5$ & $5 \times 10^{4}$ & 0 & 0 & 0 \\
SX-111 & $44.5-47.5$ & $5 \times 10^{5}$ & 0 & 0 & 0 \\
SX-111 & $44.5-47.5$ & $5 \times 10^{6}$ & 0 & 0 & 0 \\
SX-111 & $44.5-47.5$ & $5 \times 10^{7}$ & 0 & 0 & 0 \\
SX-111 & $44.5-47.5$ & $5 \times 10^{8}$ & 0 & 0 & 0 \\
SX-111 & $47.5-50.5$ & $5 \times 10^{1}$ & 0 & 0 & 0 \\
SX-111 & $47.5-50.5$ & $5 \times 10^{2}$ & 0 & 0 & 0 \\
SX-111 & $47.5-50.5$ & $5 \times 10^{3}$ & 0 & 0 & 0 \\
SX-111 & $47.5-50.5$ & $5 \times 10^{4}$ & 0 & 0 & 0 \\
SX-111 & $47.5-50.5$ & $5 \times 10^{5}$ & 0 & 0 & 0 \\
SX-111 & $47.5-50.5$ & $5 \times 10^{6}$ & 0 & 0 & 0 \\
SX-111 & $47.5-50.5$ & $5 \times 10^{7}$ & 0 & 0 & 0 \\
SX-111 & $47.5-50.5$ & $5 \times 10^{8}$ & 0 & 0 & 0 \\
\hline
\end{tabular}


Table 13. SX-112 inventory of soil volumes, total activities and upper 95\% confidence limit, exceeding 8 activity thresholds over 17 depth increments.

\begin{tabular}{|c|c|c|c|c|c|}
\hline Tank & $\begin{array}{l}\text { Depth } \\
\text { (m) }\end{array}$ & $\begin{array}{l}\text { Threshold } \\
\text { (pCi/g) }\end{array}$ & Soil & $\begin{array}{l}\text { Total } \\
\text { (pCi) }\end{array}$ & $\begin{array}{l}\text { Upper } \\
\text { (pci) }\end{array}$ \\
\hline $\mathrm{sX}-112$ & $0.0-2.5$ & $5 \times 10^{1}$ & 0 & 0 & 0 \\
\hline SX-112 & $0.0-2.5$ & $5 \times 10^{2}$ & 0 & 0 & 0 \\
\hline$S X-112$ & $0.0-2.5$ & $5 \times 10^{3}$ & 0 & 0 & 0 \\
\hline$S X-112$ & $0.0-2.5$ & $5 \times 10^{4}$ & 0 & 0 & 0 \\
\hline$S X-112$ & $0.0-2.5$ & $5 \times 10^{5}$ & 0 & 0 & 0 \\
\hline$S X-112$ & $0.0-2.5$ & $5 \times 10^{6}$ & 0 & 0 & 0 \\
\hline$S X-112$ & $0.0-2.5$ & $5 \times 10^{7}$ & 0 & 0 & 0 \\
\hline$S X-112$ & $0.0-2.5$ & $5 \times 10^{8}$ & 0 & 0 & 0 \\
\hline$S X-112$ & $2.5-5.5$ & $5 \times 10^{1}$ & 0 & 0 & 0 \\
\hline SX-112 & $2.5-5.5$ & $5 \times 10^{2}$ & 0 & 0 & 0 \\
\hline$S X-112$ & $2.5-5.5$ & $5 \times 10^{3}$ & 0 & 0 & 0 \\
\hline$S X-112$ & $2.5-5.5$ & $5 \times 10^{4}$ & 0 & 0 & 0 \\
\hline$S X-112$ & $2.5-5.5$ & $5 \times 10^{5}$ & 0 & 0 & 0 \\
\hline$s X-112$ & $2.5-5.5$ & $5 \times 10^{6}$ & 0 & 0 & 0 \\
\hline$S X-112$ & $2.5-5.5$ & $5 \times 10^{7}$ & 0 & 0 & 0 \\
\hline $5 X-112$ & $2.5-5.5$ & $5 \times 10^{8}$ & 0 & 0 & 0 \\
\hline$S X-112$ & $5.5-8.5$ & $5 \times 10^{1}$ & 0 & 0 & 0 \\
\hline$S X-112$ & $5.5-8.5$ & $5 \times 10^{2}$ & 0 & 0 & 0 \\
\hline$S X-112$ & $5.5-8.5$ & $5 \times 10^{3}$ & 0 & 0 & 0 \\
\hline$S X-112$ & $5.5-8.5$ & $5 \times 10^{4}$ & 0 & 0 & 0 \\
\hline$S X-112$ & $5.5-8.5$ & $5 \times 10^{5}$ & 0 & 0 & 0 \\
\hline$S X-112$ & $5.5-8.5$ & $5 \times 10^{6}$ & 0 & 0 & 0 \\
\hline$S X-112$ & $5.5-8.5$ & $5 \times 10^{7}$ & 0 & 0 & 0 \\
\hline$S X-112$ & $5.5-8.5$ & $5 \times 10^{8}$ & 0 & 0 & 0 \\
\hline$S X-112$ & $8.5-11.5$ & $5 \times 10^{1}$ & 0 & 0 & 0 \\
\hline$S X-112$ & $8.5-11.5$ & $5 \times 10^{2}$ & 0 & 0 & 0 \\
\hline$S x-112$ & $8.5-11.5$ & $5 \times 10^{3}$ & 0 & 0 & 0 \\
\hline$S X-112$ & $8.5-11.5$ & $5 \times 10^{4}$ & 0 & 0 & 0 \\
\hline$S X-112$ & $8.5-11.5$ & $5 \times 10^{5}$ & 0 & 0 & 0 \\
\hline$S X-112$ & $8.5-11.5$ & $5 \times 10^{6}$ & 0 & 0 & 0 \\
\hline$S X-112$ & $8.5-11.5$ & $5 \times 10^{7}$ & 0 & 0 & 0 \\
\hline$S X-112$ & $8.5-11.5$ & $5 \times 10^{8}$ & 0 & 0 & 0 \\
\hline$S X-112$ & $11.5-14.5$ & $5 \times 10^{1}$ & 0 & 0 & 0 \\
\hline$S x-112$ & $11.5-14.5$ & $5 \times 10^{2}$ & 0 & 0 & 0 \\
\hline$S X-112$ & $11.5-14.5$ & $5 \times 10^{3}$ & 0 & 0 & 0 \\
\hline SX-112 & $11.5-14.5$ & $5 \times 10^{4}$ & 0 & 0 & 0 \\
\hline$S X-112$ & $11.5-14.5$ & $5 \times 10^{5}$ & 0 & 0 & 0 \\
\hline$s X-112$ & $11.5-14.5$ & $5 \times 10^{6}$ & 0 & 0 & 0 \\
\hline$s X-112$ & $11.5-14.5$ & $5 \times 10^{7}$ & 0 & 0 & 0 \\
\hline$S X-112$ & $11.5-14.5$ & $5 \times 10^{8}$ & 0 & 0 & 0 \\
\hline SX-112 & $14.5-17.5$ & $5 \times 10^{1}$ & 0 & 0 & 0 \\
\hline$S X-112$ & $14.5-17.5$ & $5 \times 10^{2}$ & 0 & 0 & 0 \\
\hline$S X-112$ & $14.5-17.5$ & $5 \times 10^{3}$ & 0 & 0 & 0 \\
\hline $5 X-112$ & $14.5-17.5$ & $5 \times 10^{4}$ & 0 & 0 & 0 \\
\hline$s x-112$ & $14.5-17.5$ & $5 \times 10^{5}$ & 0 & 0 & 0 \\
\hline$S X-112$ & $14.5-17.5$ & $5 \times 10^{6}$ & 0 & 0 & 0 \\
\hline SX-112 & $14.5-17.5$ & $5 \times 10^{7}$ & 0 & 0 & 0 \\
\hline$S X-112$ & $14.5-17.5$ & $5 \times 10^{8}$ & 0 & 0 & 0 \\
\hline$S X-112$ & $17.5-20.5$ & $5 \times 10^{1}$ & 5 & $6.59550 E+08$ & $3.67724 \mathrm{E}+09$ \\
\hline$S X-112$ & $17.5-20.5$ & $5 \times 10^{2}$ & 0 & 0 & 0 \\
\hline$S X-112$ & $17.5-20.5$ & $5 \times 10^{3}$ & 0 & 0 & 0 \\
\hline SX-112 & $17.5-20.5$ & $5 \times 10^{4}$ & 0 & 0 & 0 \\
\hline sX-112 & $17.5-20.5$ & $5 \times 10^{5}$ & 0 & 0 & 0 \\
\hline$S X-112$ & $17.5-20.5$ & $5 \times 10^{6}$ & 0 & 0 & 0 \\
\hline$S X-112$ & $17.5-20.5$ & $5 \times 10^{7}$ & 0 & 0 & 0 \\
\hline$s X-112$ & $17.5-20.5$ & $5 \times 10^{8}$ & 0 & 0 & 0 \\
\hline$s X-112$ & $20.5-23.5$ & $5 \times 10^{1}$ & 236 & $3.97563 E+11$ & $1: 96114 \mathrm{E}+12$ \\
\hline$s X-112$ & $20.5-23.5$ & $5 \times 10^{2}$ & 92 & $3.60181 E+11$ & $1.81149 \mathrm{E}+12$ \\
\hline$S X-112$ & $20.5-23.5$ & $5 \times 10^{3}$ & 9 & $1.03884 \mathrm{E}+11$ & $5.69996 \mathrm{E}+11$ \\
\hline$S X-112$ & $20.5-23.5$ & $5 \times 10^{4}$ & 0 & 0 & 0 \\
\hline
\end{tabular}


Table 13. Continued.

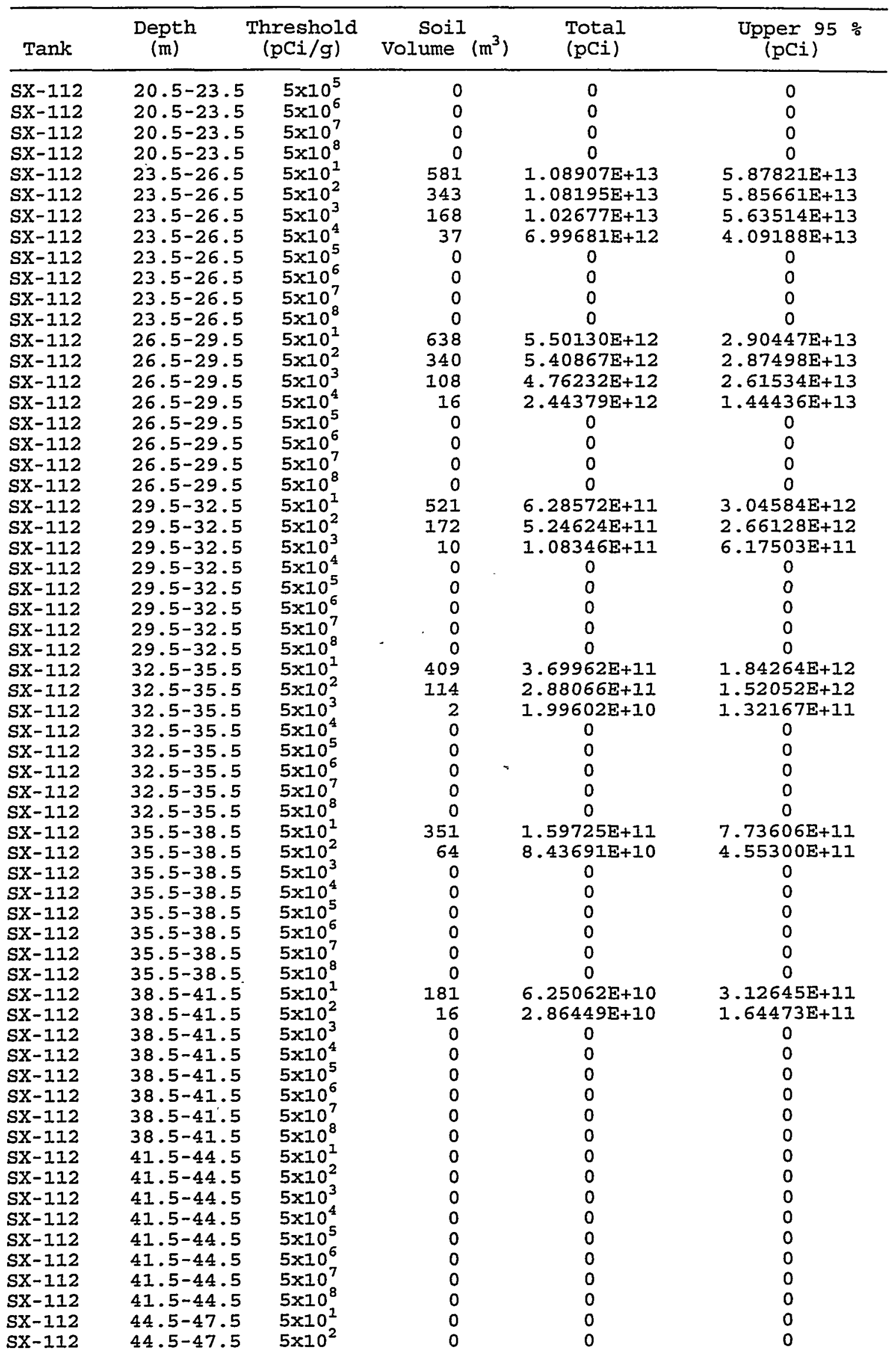


HNF-5782 Rev. 0

Table 13. Continued.

\begin{tabular}{cccccc}
\hline Tank & $\begin{array}{c}\text { Depth } \\
(\mathrm{m})\end{array}$ & $\begin{array}{c}\text { Threshold } \\
(\mathrm{pCi} / \mathrm{g})\end{array}$ & $\begin{array}{c}\text { Soil } \\
\text { Volume } \\
\left(\mathrm{m}^{3}\right)\end{array}$ & $\begin{array}{c}\text { Total } \\
\text { (pCi) }\end{array}$ & $\begin{array}{c}\text { Upper 95 } \\
\text { (pCi) }\end{array}$ \\
\hline $\mathrm{SX}-112$ & $44.5-47.5$ & $5 \times 10^{3}$ & 0 & 0 & 0 \\
$\mathrm{SX}-112$ & $44.5-47.5$ & $5 \times 10^{4}$ & 0 & 0 & 0 \\
$\mathrm{SX}-112$ & $44.5-47.5$ & $5 \times 10^{5}$ & 0 & 0 & 0 \\
$\mathrm{SX}-112$ & $44.5-47.5$ & $5 \times 10^{6}$ & 0 & 0 & 0 \\
$\mathrm{SX}-112$ & $44.5-47.5$ & $5 \times 10^{7}$ & 0 & 0 & 0 \\
$\mathrm{SX}-112$ & $44.5-47.5$ & $5 \times 10^{8}$ & 0 & 0 & 0 \\
$\mathrm{SX}-112$ & $47.5-50.5$ & $5 \times 10^{1}$ & 0 & 0 & 0 \\
$\mathrm{SX}-112$ & $47.5-50.5$ & $5 \times 10^{2}$ & 0 & 0 & 0 \\
$\mathrm{SX}-112$ & $47.5-50.5$ & $5 \times 10^{3}$ & 0 & 0 & 0 \\
$\mathrm{SX}-112$ & $47.5-50.5$ & $5 \times 10^{4}$ & 0 & 0 & 0 \\
$\mathrm{SX}-112$ & $47.5-50.5$ & $5 \times 10^{5}$ & 0 & 0 & 0 \\
$\mathrm{SX}-112$ & $47.5-50.5$ & $5 \times 10^{6}$ & 0 & 0 & 0 \\
$\mathrm{SX}-112$ & $47.5-50.5$ & $5 \times 10^{7}$ & 0 & 0 & 0 \\
$\mathrm{SX}-112$ & $47.5-50.5$ & $5 \times 10^{8}$ & 0 & 0 & 0 \\
\hline
\end{tabular}


Table 14. SX-113 inventory of soil volumes, total activities and upper 95\% confidence limit, exceeding 8 activity thresholds over 17 depth increments.

\begin{tabular}{|c|c|c|c|c|c|}
\hline Tank & $\begin{array}{l}\text { Depth } \\
\text { (m) }\end{array}$ & $\begin{array}{l}\text { Threshold } \\
\text { (pCi/g) }\end{array}$ & Soil & $\begin{array}{l}\text { Total } \\
\text { (pci) }\end{array}$ & $\begin{array}{l}\text { Upper } 95 \text { \% } \\
\text { (pCi) }\end{array}$ \\
\hline$s X-113$ & $0.0-2.5$ & $5 \times 10^{1}$ & 0 & 0 & 0 \\
\hline$S X-113$ & $0.0-2.5$ & $5 \times 10^{2}$ & 0 & 0 & 0 \\
\hline$s x-113$ & $0.0-2.5$ & $5 \times 10^{3}$ & 0 & 0 & 0 \\
\hline $5 X-113$ & $0.0-2.5$ & $5 \times 10^{4}$ & 0 & 0 & 0 \\
\hline$s X-113$ & $0.0-2.5$ & $5 \times 10^{5}$ & 0 & 0 & 0 \\
\hline$s x-113$ & $0.0-2.5$ & $5 \times 10_{7}^{6}$ & 0 & 0 & 0 \\
\hline$s x-113$ & $0.0-2.5$ & $5 \times 10^{7}$ & 0 & 0 & 0 \\
\hline$s x-113$ & $0.0-2.5$ & $5 \times 10^{8}$ & 0 & 0 & 0 \\
\hline $5 X-113$ & $2.5-5.5$ & $5 \times 10^{1}$ & 0 & 0 & 0 \\
\hline$s x-113$ & $2.5-5.5$ & $5 \times 10^{2}$ & 0 & 0 & 0 \\
\hline$s x-113$ & $2.5-5.5$ & $5 \times 10^{3}$ & 0 & 0 & 0 \\
\hline $5 X-113$ & $2.5-5.5$ & $5 \times 10^{4}$ & 0 & 0 & 0 \\
\hline$s x-113$ & $2.5-5.5$ & $5 \times 10^{5}$ & 0 & 0 & 0 \\
\hline$s x-113$ & $2.5-5.5$ & $5 \times 10^{6}$ & 0 & 0 & 0 \\
\hline$s x-113$ & $2.5-5.5$ & $5 \times 10^{7}$ & 0 & 0 & 0 \\
\hline$s x-113$ & $2.5-5.5$ & $5 \times 10^{8}$ & 0 & 0 & 0 \\
\hline sX-113 & $5.5-8.5$ & $5 \times 10^{1}$ & 0 & 0 & 0 \\
\hline$S X-113$ & $5.5-8.5$ & $5 \times 10^{2}$ & 0 & 0 & 0 \\
\hline$S X-113$ & $5.5-8.5$ & $5 \times 10^{3}$ & 0 & 0 & 0 \\
\hline$s x-113$ & $5.5-8.5$ & $5 \times 10^{4}$ & 0 & 0 & 0 \\
\hline $5 X-113$ & $5.5-8.5$ & $5 \times 10^{5}$ & 0 & 0 & 0 \\
\hline $\mathrm{sx}-113$ & $5.5-8.5$ & $5 \times 10^{6}$ & 0 & 0 & 0 \\
\hline$s X-113$ & $5.5-8.5$ & $5 \times 10^{7}$ & 0 & 0 & 0 \\
\hline$S X-113$ & $5.5-8.5$ & $5 \times 10^{8}$ & 0 & 0 & 0 \\
\hline $5 x-113$ & $8.5-11.5$ & $5 \times 10^{1}$ & 0 & 0 & 0 \\
\hline $5 x-113$ & $8.5-11.5$ & $5 \times 10^{2}$ & 0 & 0 & 0 \\
\hline $5 x-113$ & $8.5-11.5$ & $5 \times 10^{3}$ & 0 & 0 & 0 \\
\hline$s X-113$ & $8.5-11.5$ & $5 \times 10^{4}$ & 0 & 0 & 0 \\
\hline$s X-113$ & $8.5-11.5$ & $5 \times 10^{5}$ & 0 & 0 & 0 \\
\hline $5 x-113$ & $8.5-11.5$ & $5 \times 10^{6}$ & 0 & 0 & 0 \\
\hline$s x-113$ & $8 \cdot 5-11.5$ & $5 \times 10^{7}$ & 0 & 0 & 0 \\
\hline$s x-113$ & $8.5-11.5$ & $5 \times 10^{8}$ & 0 & 0 & 0 \\
\hline $5 X-113$ & $11.5-14.5$ & $5 \times 10^{1}$ & 0 & 0 & 0 \\
\hline$S X-113$ & $11.5-14.5$ & $5 \times 10^{2}$ & 0 & 0 & 0 \\
\hline$s X-113$ & $11.5-14.5$ & $5 \times 10^{3}$ & 0 & 0 & 0 \\
\hline $5 x-113$ & $11.5-14.5$ & $5 \times 10^{4}$ & 0 & 0 & 0 \\
\hline$s x-113$ & $11.5-14.5$ & $5 \times 10^{5}$ & 0 & 0 & $\begin{array}{l}0 \\
0\end{array}$ \\
\hline$s x-113$ & $11.5-14.5$ & $5 \times 10^{6}$ & 0 & 0 & $\begin{array}{l}0 \\
0\end{array}$ \\
\hline $5 X-113$ & $11.5-14.5$ & $5 \times 10^{7}$ & 0 & 0 & $\begin{array}{l}0 \\
0\end{array}$ \\
\hline$s X-113$ & $11.5-14.5$ & $5 \times 10^{8}$ & 0 & 0 & 0 \\
\hline $5 X-113$ & $\begin{array}{l}11.5-14.5 \\
14.5-17.5\end{array}$ & $5 \times 10$ & 0 & 0 & 0 \\
\hline$s X-113$ & $\begin{array}{l}14 \cdot 5-17 \cdot 5 \\
14.5-17 \cdot 5\end{array}$ & $5 \times 10$ & 0 & 0 & 0 \\
\hline $5 x-113$ & $14 \cdot 5-17 \cdot 5$ & $5 \times 10^{2}$ & 0 & 0 & 0 \\
\hline $5 x-113$ & $14.5-17 \cdot 5$ & $5 \times 10^{-}$ & 0 & 0 & 0 \\
\hline$s x-113$ & $14 \cdot 5-17.5$ & $5 \times 10^{2}$ & 0 & 0 & 0 \\
\hline$s x-113$ & $14.5-17.5$ & $5 \times 10^{-}$ & 0 & 0 & 0 \\
\hline & $14.5-17.5$ & $5 \times 10^{\circ}$ & 0 & 0 & 0 \\
\hline$s X-113$ & $14.5-17.5$ & $5 \times 10^{\prime}$ & 0 & 0 & 0 \\
\hline$S X-113$ & $14.5-17.5$ & $5 \times 10^{8}$ & 0 & 0 & 0 \\
\hline$s x-113$ & $17.5-20.5$ & $5 \times 10^{1}$ & 0 & 0 & 0 \\
\hline$s x-113$ & $17.5-20.5$ & $5 \times 10^{2}$ & 0 & 0 & 0 \\
\hline$s x-113$ & $17.5-20.5$ & $5 \times 10^{3}$ & 0 & 0 & 0 \\
\hline $5 x-113$ & $17.5-20.5$ & $5 \times 10^{4}$ & 0 & 0 & 0 \\
\hline $5 x-113$ & $17.5-20.5$ & $5 \times 10^{5}$ & 0 & 0 & 0 \\
\hline $5 X-113$ & $17.5-20.5$ & $5 \times 10^{6}$ & 0 & 0 & 0 \\
\hline $5 X-113$ & $17.5-20.5$ & $5 \times 10^{7}$ & 0 & 0 & 0 \\
\hline $5 x-113$ & $17.5-20.5$ & $5 \times 10^{8}$ & 0 & 0 & 0 \\
\hline $5 x-113$ & $20.5-23.5$ & $5 \times 10^{1}$ & 0 & 0 & 0 \\
\hline$s x-113$ & $20.5-23.5$ & $5 \times 10^{2}$ & 0 & 0 & 0 \\
\hline$s x-113$ & $20.5-23.5$ & $5 \times 10^{3}$ & 0 & 0 & 0 \\
\hline$s x-113$ & $20.5-23.5$ & $5 \times 10^{4}$ & 0 & 0 & 0 \\
\hline
\end{tabular}


Table 14. Continued.

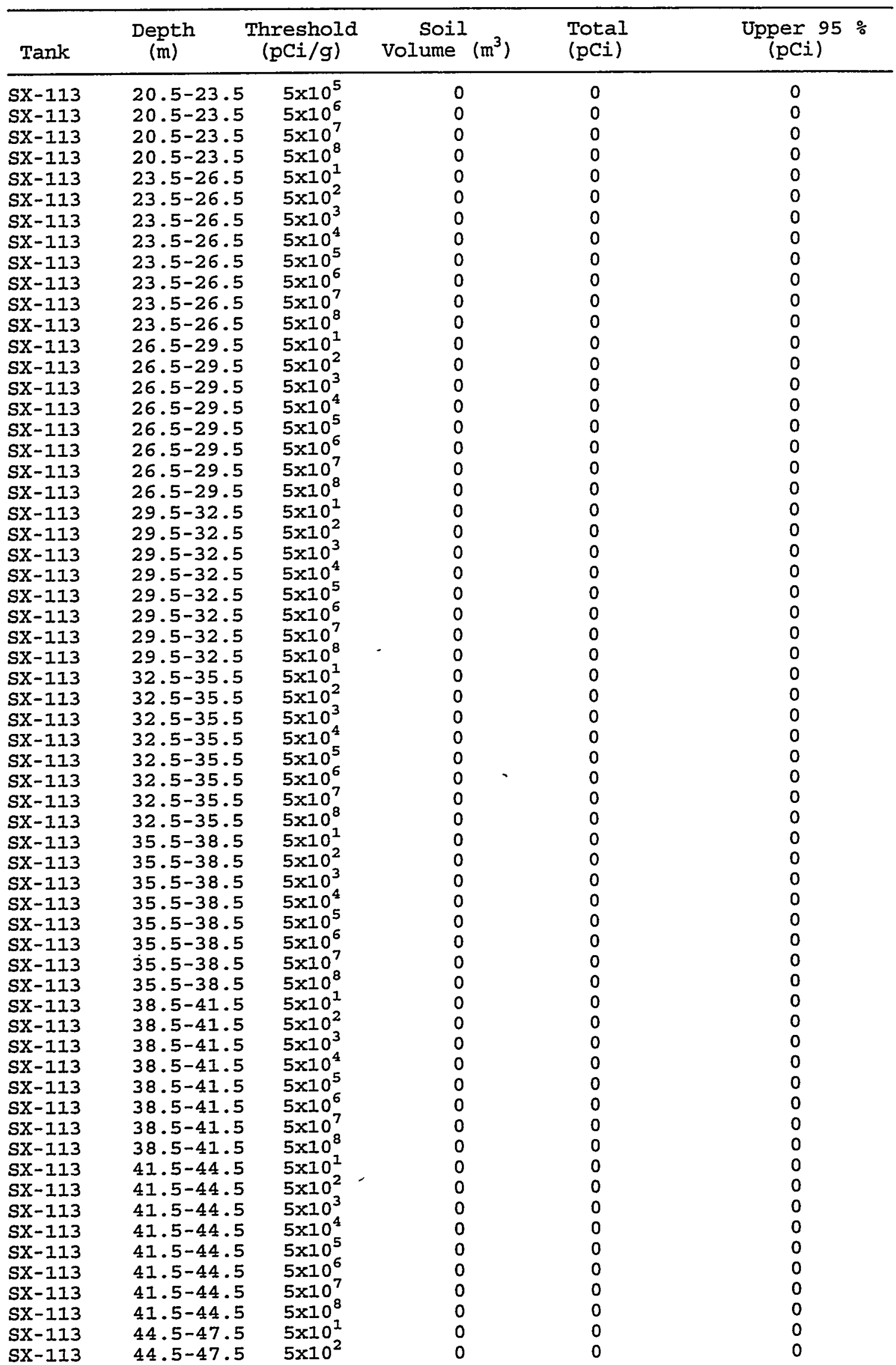


Table 14. Continued.

\begin{tabular}{|c|c|c|c|c|c|}
\hline Tank & $\begin{array}{l}\text { Depth } \\
\text { (m) }\end{array}$ & $\begin{array}{l}\text { Threshold } \\
\text { (pci/g) }\end{array}$ & Soil & $\begin{array}{l}\text { Total } \\
\text { (pCi) }\end{array}$ & $\begin{array}{l}\text { Upper } 95 \% \\
\text { (pCi) }\end{array}$ \\
\hline $\begin{array}{l}s X-113 \\
s X-113 \\
s X-113 \\
s X-113 \\
s X-113 \\
s X-113 \\
s X-113 \\
s X-113 \\
s X-113 \\
s X-113 \\
s X-113 \\
s X-113 \\
s X-113 \\
s X-113\end{array}$ & $\begin{array}{l}44 \cdot 5-47 \cdot 5 \\
44 \cdot 5-47 \cdot 5 \\
44 \cdot 5-47 \cdot 5 \\
44 \cdot 5-47 \cdot 5 \\
44 \cdot 5-47 \cdot 5 \\
44 \cdot 5-47 \cdot 5 \\
47 \cdot 5-50 \cdot 5 \\
47 \cdot 5-50 \cdot 5 \\
47 \cdot 5-50 \cdot 5 \\
47 \cdot 5-50 \cdot 5 \\
47 \cdot 5-50 \cdot 5 \\
47 \cdot 5-50 \cdot 5 \\
47 \cdot 5-50 \cdot 5 \\
47 \cdot 5-50 \cdot 5\end{array}$ & $\begin{array}{l}5 \times 10^{3} \\
5 \times 10^{4} \\
5 \times 10^{5} \\
5 \times 10^{6} \\
5 \times 10^{7} \\
5 \times 10^{8} \\
5 \times 10^{1} \\
5 \times 10^{2} \\
5 \times 10^{3} \\
5 \times 10^{4} \\
5 \times 10^{5} \\
5 \times 10^{6} \\
5 \times 10^{7} \\
5 \times 10^{8}\end{array}$ & $\begin{array}{l}0 \\
0 \\
0 \\
0 \\
0 \\
0 \\
0 \\
0 \\
0 \\
0 \\
0 \\
0 \\
0 \\
0\end{array}$ & $\begin{array}{l}0 \\
0 \\
0 \\
0 \\
0 \\
0 \\
0 \\
0 \\
0 \\
0 \\
0 \\
0 \\
0 \\
0\end{array}$ & $\begin{array}{l}0 \\
0 \\
0 \\
0 \\
0 \\
0 \\
0 \\
0 \\
0 \\
0 \\
0 \\
0 \\
0 \\
0\end{array}$ \\
\hline
\end{tabular}


Table 15. SX-114 inventory of soil volumes, total activities and upper 95\% confidence limit, exceeding 8 activity thresholds over 17 depth increments.

\begin{tabular}{|c|c|c|c|c|c|}
\hline Tank & $\begin{array}{l}\text { Depth } \\
(\mathrm{m})\end{array}$ & $\begin{array}{l}\text { Threshold } \\
\text { (pCi/g) }\end{array}$ & Volume $\left(\mathrm{m}^{3}\right)$ & $\begin{array}{l}\text { Total } \\
\text { (pCi) }\end{array}$ & $\begin{array}{l}\text { Upper } 95 \\
\text { (pCi) }\end{array}$ \\
\hline$S X-114$ & $0.0-2.5$ & $5 \times 10^{1}$ & 0 & 0 & 0 \\
\hline$S X-114$ & $0.0-2.5$ & $5 \times 10^{2}$ & 0 & 0 & 0 \\
\hline$S X-114$ & $0.0-2.5$ & $5 \times 10^{3}$ & 0 & 0 & 0 \\
\hline$S X-114$ & $0.0-2.5$ & $5 \times 10^{4}$ & 0 & 0 & 0 \\
\hline SX-114 & $0.0-2.5$ & $5 \times 10^{5}$ & 0 & 0 & 0 \\
\hline$s X-114$ & $0.0-2.5$ & $5 \times 10^{6}$ & 0 & 0 & 0 \\
\hline SX-114 & $0.0-2.5$ & $5 \times 10^{7}$ & 0 & 0 & 0 \\
\hline SX-114 & $0.0-2.5$ & $5 \times 10^{8}$ & 0 & 0 & 0 \\
\hline SX-114 & $2.5-5.5$ & $5 \times 10^{1}$ & 0 & 0 & 0 \\
\hline$S X-114$ & $2.5-5.5$ & $5 \times 10^{2}$ & 0 & 0 & 0 \\
\hline$S X-114$ & $2.5-5.5$ & $5 \times 10^{3}$ & 0 & 0 & 0 \\
\hline$S X-114$ & $2.5-5.5$ & $5 \times 10^{4}$ & 0 & 0 & 0 \\
\hline $5 X-114$ & $2.5-5.5$ & $5 \times 10^{5}$ & 0 & 0 & 0 \\
\hline$S X-114$ & $2.5-5.5$ & $5 \times 10^{6}$ & 0 & 0 & 0 \\
\hline$S X-114$ & $2.5-5.5$ & $5 \times 10^{7}$ & 0 & 0 & 0 \\
\hline$S X-114$ & $2.5-5.5$ & $5 \times 10^{8}$ & 0 & 0 & 0 \\
\hline$S X-114$ & $5.5-8.5$ & $5 \times 10^{1}$ & 0 & 0 & 0 \\
\hline SX-114 & $5.5-8.5$ & $5 \times 10^{2}$ & 0 & 0 & 0 \\
\hline $5 X-114$ & $5.5-8.5$ & $5 \times 10^{3}$ & 0 & 0 & 0 \\
\hline$S X-114$ & $5.5-8.5$ & $5 \times 10^{4}$ & 0 & 0 & 0 \\
\hline$S X-114$ & $5.5-8.5$ & $5 \times 10^{5}$ & 0 & 0 & 0 \\
\hline$S X-114$ & $5.5-8.5$ & $5 \times 10^{6}$ & 0 & 0 & 0 \\
\hline$S X-114$ & $5.5-8.5$ & $5 \times 10^{7}$ & 0 & 0 & 0 \\
\hline$s X-114$ & $5.5-8.5$ & $5 \times 10^{8}$ & 0 & 0 & 0 \\
\hline$S X-114$ & $8.5-11.5$ & $5 \times 10^{1}$ & 0 & 0 & 0 \\
\hline$S X-114$ & $8.5-11.5$ & $5 \times 10^{2}$ & 0 & 0 & 0 \\
\hline$S X-114$ & $8.5-11.5$ & $5 \times 10^{3}$ & 0 & 0 & 0 \\
\hline$S X-114$ & $8.5-11.5$ & $5 \times 10^{4}$ & 0 & 0 & 0 \\
\hline$S X-114$ & $8.5-11.5$ & $5 \times 10^{5}$ & 0 & 0 & 0 \\
\hline$S X-114$ & $8.5-11.5$ & $5 \times 10^{6}$ & 0 & 0 & 0 \\
\hline$S X-114$ & $8.5-11.5$ & $5 \times 10^{7}$ & 0 & 0 & 0 \\
\hline$S X-114$ & $8.5-11.5$ & $5 \times 10^{8}$ & 0 & 0 & 0 \\
\hline$s X-114$ & $11.5-14.5$ & $5 \times 10^{1}$ & 0 & 0 & 0 \\
\hline$S X-114$ & $11.5-14.5$ & $5 \times 10^{2}$ & 0 & 0 & 0 \\
\hline$s X-114$ & $11.5-14.5$ & $5 \times 10^{3}$ & 0 & 0 & 0 \\
\hline$s x-114$ & $11.5-14.5$ & $5 \times 10^{4}$ & 0 & 0 & 0 \\
\hline$S X-114$ & $11.5-14.5$ & $5 \times 10^{5}$ & 0 & 0 & $\begin{array}{l}0 \\
0\end{array}$ \\
\hline$s X-114$ & $11 \cdot 5-14.5$ & $5 \times 10^{6}$ & 0 & 0 & $\begin{array}{l}0 \\
0\end{array}$ \\
\hline $5 x-114$ & $11.5-14.5$ & $5 \times 10^{7}$ & 0 & 0 & 0 \\
\hline & & & 0 & 0 & 0 \\
\hline SX-114 & $11 \cdot 5-14 \cdot 5$ & $5 \times 10^{\circ}$ & 0 & 0 & 0 \\
\hline$S X-114$ & $14.5-17.5$ & $5 \times 10^{1}$ & 0 & 0 & 0 \\
\hline$S X-114$ & $14.5-17.5$ & $5 \times 10^{2}$ & 0 & 0 & 0 \\
\hline$S X-114$ & $14.5-17.5$ & $5 \times 10^{3}$ & 0 & 0 & 0 \\
\hline$S X-114$ & $14.5-17.5$ & $5 \times 10^{4}$ & 0 & 0 & 0 \\
\hline$S X-114$ & $14.5-17.5$ & $5 \times 10^{5}$ & 0 & 0 & 0 \\
\hline$S X-114$ & $14.5-17.5$ & $5 \times 10^{6}$ & 0 & 0 & 0 \\
\hline $\mathrm{sX}-114$ & $14.5-17.5$ & $5 \times 10^{7}$ & 0 & 0 & 0 \\
\hline $\mathrm{SX}-114$ & $14.5-17.5$ & $5 \times 10^{8}$ & 0 & 0 & 0 \\
\hline$S X-114$ & $17.5-20.5$ & $5 \times 10^{1}$ & 0 & 0 & 0 \\
\hline $5 X-114$ & $17.5-20.5$ & $5 \times 10^{2}$ & 0 & 0 & 0 \\
\hline$S X-114$ & $17.5-20.5$ & $5 \times 10^{3}$ & 0 & 0 & 0 \\
\hline$s X-114$ & $17.5-20.5$ & $5 \times 10^{4}$ & 0 & 0 & 0 \\
\hline$s x-114$ & $17.5-20.5$ & $5 \times 10^{5}$ & 0 & 0 & 0 \\
\hline$s X-114$ & $17.5-20.5$ & $5 \times 10^{6}$ & 0 & 0 & 0 \\
\hline$S x-114$ & $17.5-20.5$ & $5 \times 10^{7}$ & 0 & 0 & 0 \\
\hline$S X-114$ & $17.5-20.5$ & $5 \times 10^{8}$ & 0 & 0 & 0 \\
\hline$S X-114$ & $20.5-23.5$ & $5 \times 10^{1}$ & 0 & 0 & 0 \\
\hline$S X-114$ & $20.5-23.5$ & $5 \times 10^{2}$ & 0 & 0 & 0 \\
\hline$s X-114$ & $20.5-23.5$ & $5 \times 10^{3}$ & 0 & 0 & 0 \\
\hline $\begin{array}{l}S X-114 \\
S X-114\end{array}$ & $20.5-23.5$ & $5 \times 10^{4}$ & 0 & 0 & 0 \\
\hline
\end{tabular}


Table 15. Continued.

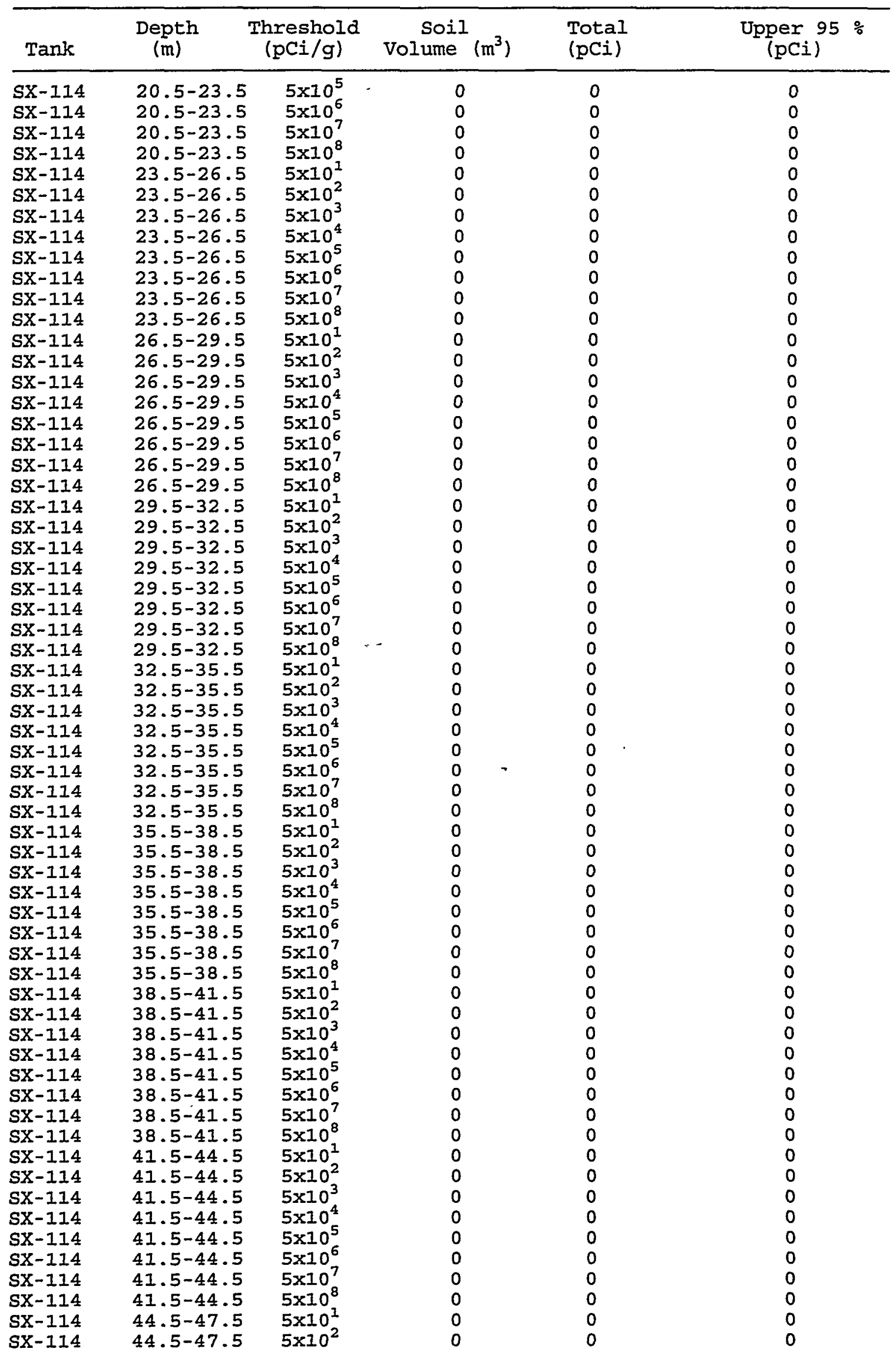


HNF-5782 Rev. 0

Table 15. Continued.

\begin{tabular}{|c|c|c|c|c|c|}
\hline Tank & $\underset{(m)}{\text { Depth }}$ & $\begin{array}{l}\text { Threshold } \\
\text { (pCi/g) }\end{array}$ & Volume $\left(\mathrm{m}^{3}\right)$ & $\begin{array}{l}\text { Total } \\
\text { (pci) }\end{array}$ & $\begin{array}{l}\text { Upper } 95 \% \\
\text { (pCi) }\end{array}$ \\
\hline $\begin{array}{l}\text { SX-114 } \\
\text { SX-114 } \\
\text { SX-114 } \\
\text { SX-114 } \\
\text { SX-114 } \\
\text { SX-114 } \\
\text { SX-114 } \\
\text { SX-114 } \\
\text { SX-114 } \\
\text { SX-114 } \\
\text { SX-114 } \\
\text { SX-114 } \\
\text { SX-114 } \\
\text { SX-114 }\end{array}$ & $\begin{array}{l}44.5-47.5 \\
44.5-47.5 \\
44.5-47.5 \\
44.5-47.5 \\
44.5-47.5 \\
44.5-47.5 \\
47.5-50.5 \\
47.5-50.5 \\
47.5-50.5 \\
47.5-50.5 \\
47.5-50.5 \\
47.5-50.5 \\
47.5-50.5 \\
47.5-50.5\end{array}$ & $\begin{array}{l}5 \times 10^{3} \\
5 \times 10^{4} \\
5 \times 10^{5} \\
5 \times 10^{6} \\
5 \times 10^{7} \\
5 \times 10^{8} \\
5 \times 10^{1} \\
5 \times 10^{2} \\
5 \times 10^{3} \\
5 \times 10^{4} \\
5 \times 10^{5} \\
5 \times 10^{6} \\
5 \times 10^{7} \\
5 \times 10^{8}\end{array}$ & $\begin{array}{l}0 \\
0 \\
0 \\
0 \\
0 \\
0 \\
0 \\
0 \\
0 \\
0 \\
0 \\
0 \\
0 \\
0\end{array}$ & $\begin{array}{l}0 \\
0 \\
0 \\
0 \\
0 \\
0 \\
0 \\
0 \\
0 \\
0 \\
0 \\
0 \\
0 \\
0\end{array}$ & $\begin{array}{l}0 \\
0 \\
0 \\
0 \\
0 \\
0 \\
0 \\
0 \\
0 \\
0 \\
0 \\
0 \\
0 \\
0\end{array}$ \\
\hline
\end{tabular}


Table 16. SX-115 inventory of soil volumes, total activities and upper 95\% confidence limit, exceeding 8 activity thresholds over 17 depth increments.

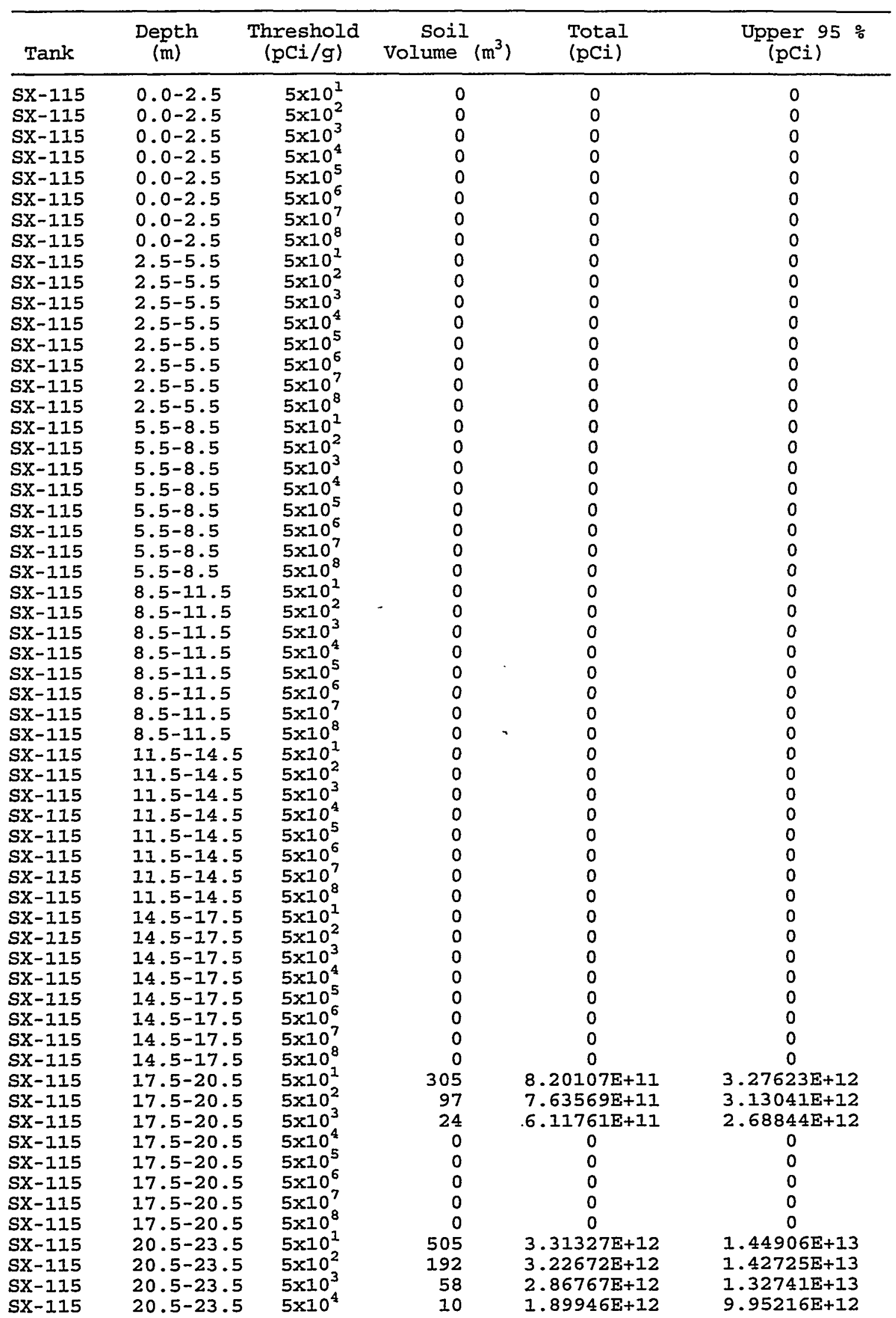


Table 16. Continued.

\begin{tabular}{|c|c|c|c|c|c|}
\hline Tank & $\begin{array}{c}\text { Depth } \\
\text { (m) }\end{array}$ & $\begin{array}{l}\text { Threshold } \\
\text { (pCi/g) }\end{array}$ & Soil & $\begin{array}{l}\text { Total } \\
\text { (pci) }\end{array}$ & $\begin{array}{l}\text { Upper } 95 \% \\
\text { (pCi) }\end{array}$ \\
\hline$S X-115$ & $20.5-23.5$ & $5 \times 10^{5}$ & 0 & 0 & 0 \\
\hline $5 X-115$ & $20.5-23.5$ & $5 \times 10^{6}$ & 0 & 0 & 0 \\
\hline$S X-115$ & $20 \cdot 5-23.5$ & $5 \times 10^{7}$ & 0 & 0 & 0 \\
\hline$S x-115$ & $20.5-23.5$ & $5 \times 10^{8}$ & 0 & 0 & 0 \\
\hline$S x-115$ & $23.5-26.5$ & $5 \times 10^{1}$ & 3 & $2.54193 E+08$ & $1.18317 \mathrm{E}+09$ \\
\hline$S x-115$ & $23.5-26.5$ & $5 \times 10^{2}$ & 0 & 0 & 0 \\
\hline$S X-115$ & $23.5-26.5$ & $5 \times 10^{3}$ & 0 & 0 & 0 \\
\hline$S X-115$ & $23.5-26.5$ & $5 \times 10^{4}$ & 0 & 0 & 0 \\
\hline $5 x-115$ & $23.5-26.5$ & $5 \times 10^{5}$ & 0 & 0 & 0 \\
\hline$S X-115$ & $23.5-26.5$ & $5 \times 10^{6}$ & 0 & 0 & 0 \\
\hline$s X-115$ & $23 \cdot 5-26.5$ & $5 \times 10^{7}$ & 0 & 0 & 0 \\
\hline$S X-115$ & $23 \cdot 5-26.5$ & $5 \times 10^{8}$ & 0 & 0 & 0 \\
\hline$S X-115$ & $26.5-29.5$ & $5 \times 10^{1}$ & 0 & 0 & 0 \\
\hline$S X-115$ & $26.5-29.5$ & $5 \times 10^{2}$ & 0 & 0 & 0 \\
\hline$s X-115$ & $26.5-29.5$ & $5 \times 10^{3}$ & 0 & 0 & 0 \\
\hline$s X-115$ & $26.5-29.5$ & $5 \times 10^{4}$ & 0 & 0 & 0 \\
\hline$s x-115$ & $26.5-29.5$ & $5 \times 10^{5}$ & 0 & 0 & 0 \\
\hline$S X-115$ & $26.5-29.5$ & $5 \times 10^{6}$ & 0 & 0 & 0 \\
\hline SX-115 & $26.5-29.5$ & $5 \times 10^{7}$ & 0 & 0 & 0 \\
\hline$S X-115$ & $26.5-29.5$ & $5 \times 10^{8}$ & 0 & 0 & 0 \\
\hline $5 X-115$ & $29.5-32.5$ & $5 \times 10^{1}$ & 0 & 0 & 0 \\
\hline$S X-115$ & $29.5-32.5$ & $5 \times 10^{2}$ & 0 & 0 & 0 \\
\hline SX-115 & $29.5-32.5$ & $5 \times 10^{3}$ & 0 & 0 & 0 \\
\hline$s x-115$ & $29.5-32.5$ & $5 \times 10^{4}$ & 0 & 0 & 0 \\
\hline $5 X-115$ & $29.5-32.5$ & $5 \times 10^{5}$ & 0 & 0 & 0 \\
\hline sX-115 & $29.5-32.5$ & $5 \times 10^{6}$ & 0 & 0 & 0 \\
\hline$S X-115$ & $29.5-32.5$ & $5 \times 10^{7}$ & 0 & 0 & 0 \\
\hline$S X-115$ & $29.5-32.5$ & $5 \times 10^{8}$ & 0 & 0 & 0 \\
\hline$s x-115$ & $32.5-35.5$ & $5 \times 10^{1}$ & 0 & 0 & 0 \\
\hline$s X-115$ & $32.5-35.5$ & $5 \times 10^{2}$ & 0 & 0 & 0 \\
\hline$s x-115$ & $32.5-35.5$ & $5 \times 10^{3}$ & 0 & 0 & 0 \\
\hline$s X-115$ & $32.5-35.5$ & $5 \times 10^{4}$ & 0 & 0 & 0 \\
\hline$s x-115$ & $32.5-35.5$ & $5 \times 10^{5}$ & 0 & 0 & 0 \\
\hline$S X-115$ & $32.5-35.5$ & $5 \times 10^{6}$ & 0 & 0 & 0 \\
\hline$S X-115$ & $32.5-35.5$ & $5 \times 10^{7}$ & 0 & 0 & 0 \\
\hline$S X-115$ & $32.5-35.5$ & $5 \times 10^{8}$ & 0 & 0 & 0 \\
\hline$S X-115$ & $35.5-38.5$ & $5 \times 10^{1}$ & 0 & 0 & 0 \\
\hline$S X-115$ & $35.5-38.5$ & $5 \times 10^{2}$ & 0 & 0 & 0 \\
\hline $\mathrm{SX}-115$ & $35.5-38.5$ & $5 \times 10^{3}$ & 0 & 0 & 0 \\
\hline $\mathrm{SX}-115$ & $35.5-38.5$ & $5 \times 10^{4}$ & 0 & 0 & 0 \\
\hline$S x-115$ & $35.5-38.5$ & $5 \times 10^{5}$ & 0 & 0 & 0 \\
\hline$S X-115$ & $35.5-38.5$ & $5 \times 10^{6}$ & 0 & 0 & 0 \\
\hline$S X-115$ & $35.5-38.5$ & $5 \times 10^{7}$ & 0 & 0 & 0 \\
\hline$S X-115$ & $35.5-38.5$ & $5 \times 10^{8}$ & 0 & 0 & 0 \\
\hline$S X-115$ & $38.5-41.5$ & $5 \times 10^{1}$ & 0 & 0 & 0 \\
\hline$s X-115$ & $38.5-41.5$ & $5 \times 10^{2}$ & 0 & 0 & 0 \\
\hline$s x-115$ & $38.5-41.5$ & $5 \times 10^{3}$ & 0 & 0 & 0 \\
\hline$S x-115$ & $38.5-41.5$ & $5 \times 10^{4}$ & 0 & 0 & 0 \\
\hline$s x-115$ & $38.5-41.5$ & $5 \times 10^{5}$ & 0 & 0 & 0 \\
\hline$s x-115$ & $38.5-41.5$ & $5 \times 10^{6}$ & 0 & 0 & $\begin{array}{l}0 \\
0\end{array}$ \\
\hline$s x-115$ & $38.5-41.5$ & $5 \times 10^{7}$ & 0 & 0 & 0 \\
\hline $5 x-115$ & $38.5-41.5$ & $5 \times 10^{8}$ & 0 & 0 & $\begin{array}{l}0 \\
0\end{array}$ \\
\hline$s x-115$ & $41,5-44.5$ & $5 \times 10^{1}$ & 0 & 0 & $\begin{array}{l}0 \\
0\end{array}$ \\
\hline$S X-115$ & $415-445$ & $5 \times 10^{2}$ & 0 & 0 & $\begin{array}{l}0 \\
0\end{array}$ \\
\hline & $475-445$ & $5 \times 10^{3}$ & 0 & 0 & $\begin{array}{l}0 \\
0\end{array}$ \\
\hline$S X-115$ & $475-44$ & $5 \times 10^{4}$ & 0 & 0 & 0 \\
\hline$S X-115$ & $475-445$ & $5 \times 10^{5}$ & 0 & 0 & 0 \\
\hline$S X-115$ & $415-44$ & $5 \times 10^{6}$ & 0 & 0 & 0 \\
\hline $\begin{array}{l}5 x-115 \\
5 x-115\end{array}$ & $41 \cdot 5-44 \cdot 5$ & $5 x+0$ & 0 & 0 & 0 \\
\hline $5 X-115$ & $41.5-44.5$ & $5 \times 10$ & 0 & 0 & 0 \\
\hline$s X-115$ & $41.5-44.5$ & $5 \times 10^{8}$ & 0 & 0 & 0 \\
\hline$S X-115$ & $44.5-47.5$ & $5 \times 10^{1}$ & 0 & 0 & 0 \\
\hline$s X-115$ & $44.5-47.5$ & $5 \times 10^{2}$ & 0 & 0 & 0 \\
\hline
\end{tabular}


HINF-5782 Rev.0

Table 16. Continued.

\begin{tabular}{cccccc}
\hline Tank & $\begin{array}{l}\text { Depth } \\
(\mathrm{m})\end{array}$ & $\begin{array}{c}\text { Threshold } \\
(\mathrm{pCi} / \mathrm{g})\end{array}$ & $\left.\begin{array}{c}\text { Soil } \\
\text { Volume }\end{array} \mathrm{m}^{3}\right)$ & $\begin{array}{c}\text { Total } \\
(\mathrm{pCi})\end{array}$ & $\begin{array}{c}\text { Upper } 95 \% \\
(\mathrm{pC} i)\end{array}$ \\
\hline SX-115 & $44.5-47.5$ & $5 \times 10^{3}$ & 0 & 0 & 0 \\
SX-115 & $44.5-47.5$ & $5 \times 10^{4}$ & 0 & 0 & 0 \\
SX-115 & $44.5-47.5$ & $5 \times 10^{5}$ & 0 & 0 & 0 \\
SX-115 & $44.5-47.5$ & $5 \times 10^{6}$ & 0 & 0 & 0 \\
SX-115 & $44.5-47.5$ & $5 \times 10^{7}$ & 0 & 0 & 0 \\
SX-115 & $44.5-47.5$ & $5 \times 10^{8}$ & 0 & 0 & 0 \\
SX-115 & $47.5-50.5$ & $5 \times 10^{1}$ & 0 & 0 & 0 \\
SX-115 & $47.5-50.5$ & $5 \times 10^{2}$ & 0 & 0 & 0 \\
SX-115 & $47.5-50.5$ & $5 \times 10^{3}$ & 0 & 0 & 0 \\
SX-115 & $47.5-50.5$ & $5 \times 10^{4}$ & 0 & 0 & 0 \\
SX-115 & $47.5-50.5$ & $5 \times 10^{5}$ & 0 & 0 & 0 \\
SX-115 & $47.5-50.5$ & $5 \times 10^{6}$ & 0 & 0 & 0 \\
SX-115 & $47.5-50.5$ & $5 \times 10^{7}$ & 0 & 0 & 0 \\
SX-115 & $47.5-50.5$ & $5 \times 10^{8}$ & 0 & 0 & 0 \\
\hline
\end{tabular}


HNF-5782 Rev. 0
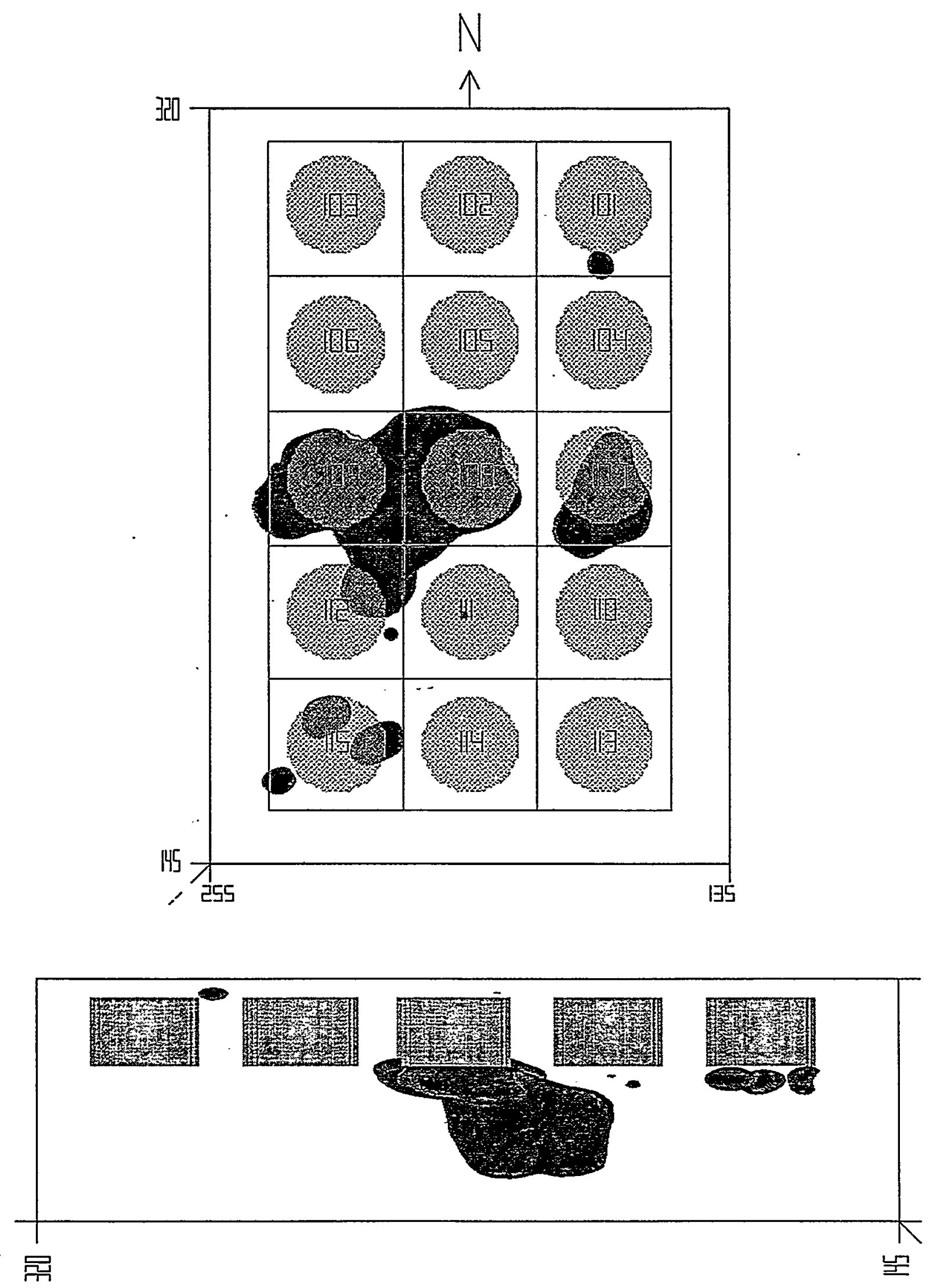

Figure 1. SX kriging point predictions exceeding $5 \times 10^{1} \mathrm{pCi} / \mathrm{g}$ from a top and west view. 

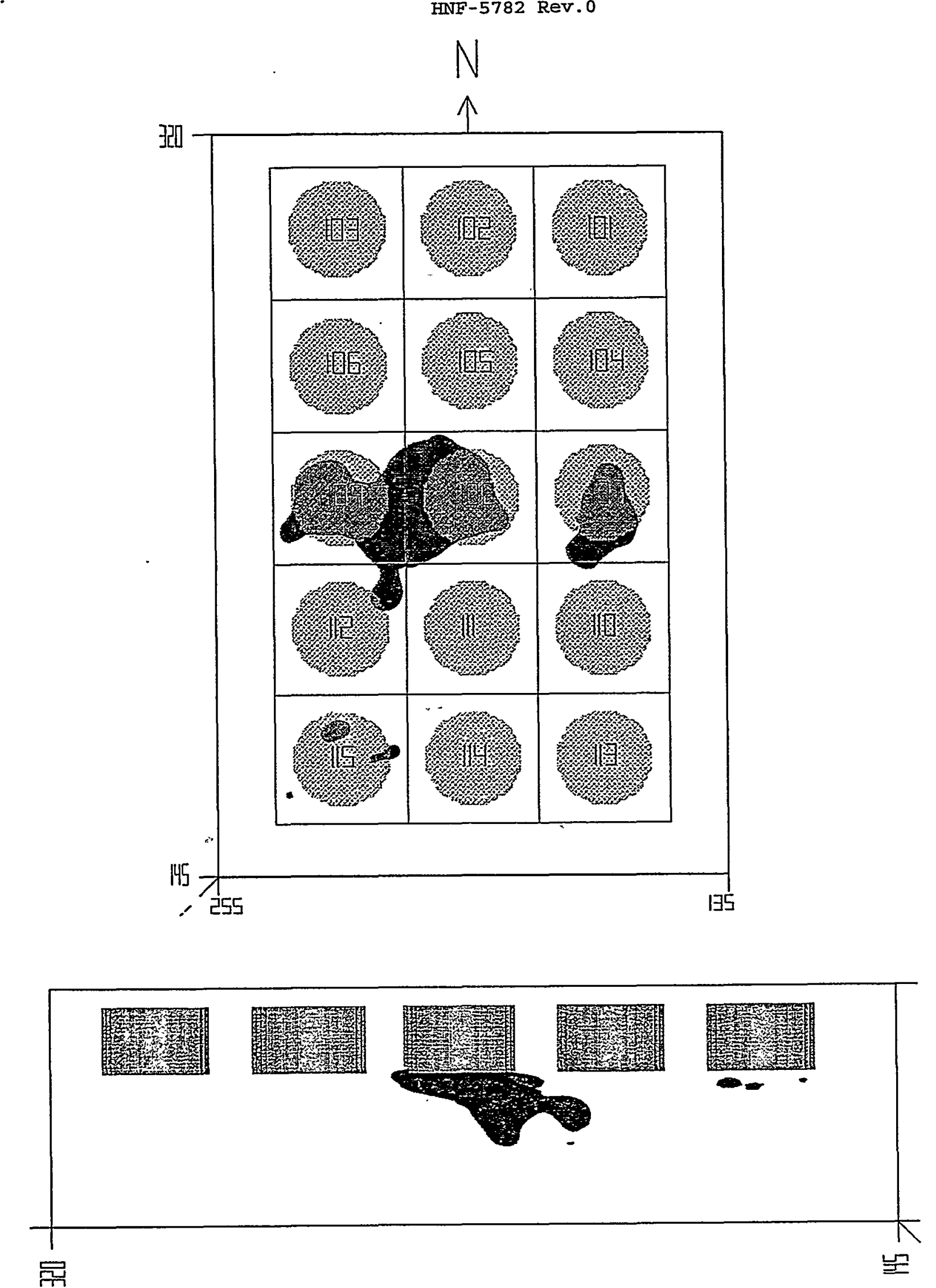

Figure 3. SX kriging point predictions exceeding $5 \times 10^{3} \mathrm{pCi} / \mathrm{g}$ from a top and west view. 

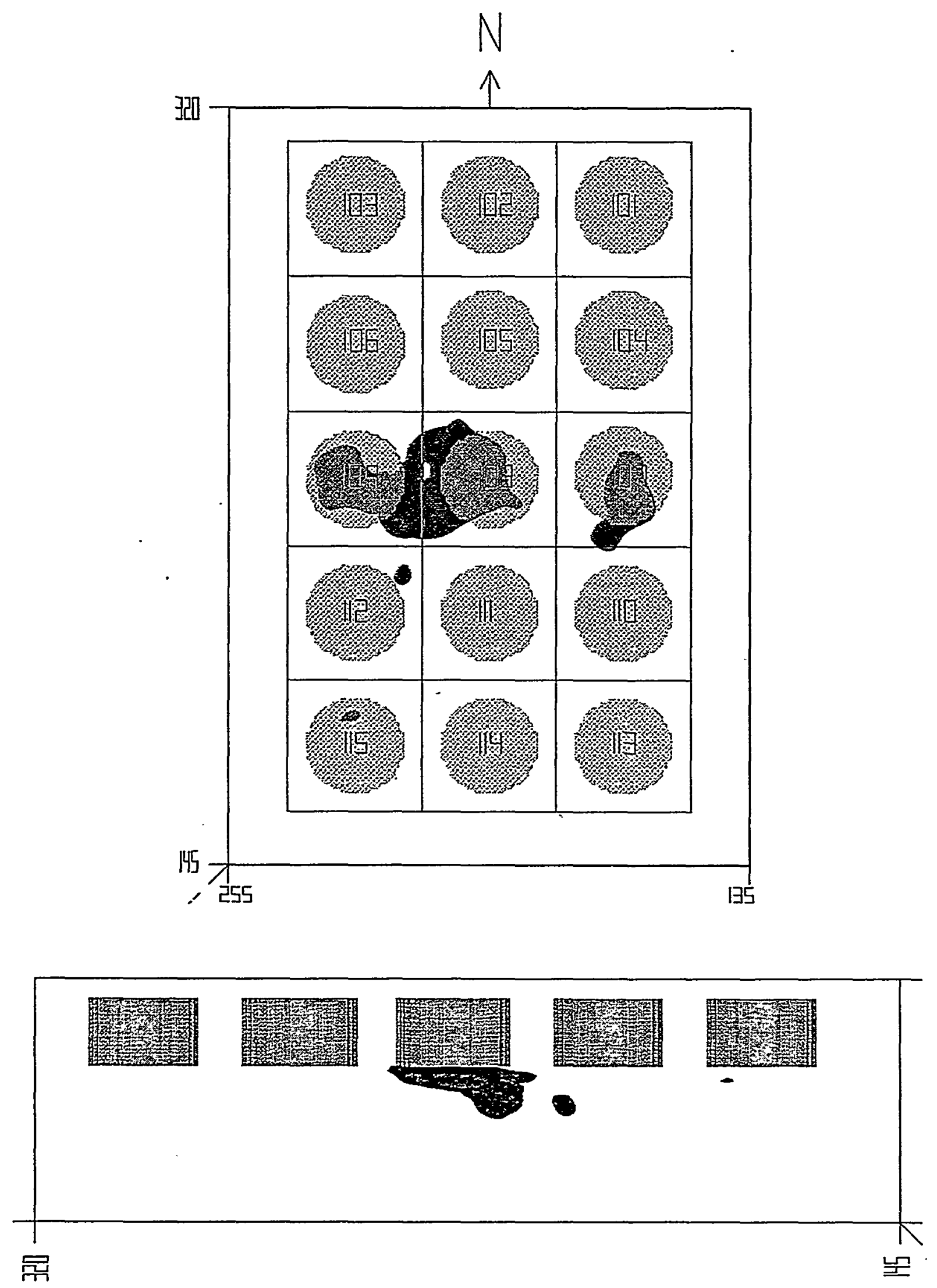

Figure 4. SX kriging point predictions exceeding $5 \times 10^{4} \mathrm{pCi} / \mathrm{g}$ from a top and west view. 
HNF-5782 Rev.0

N
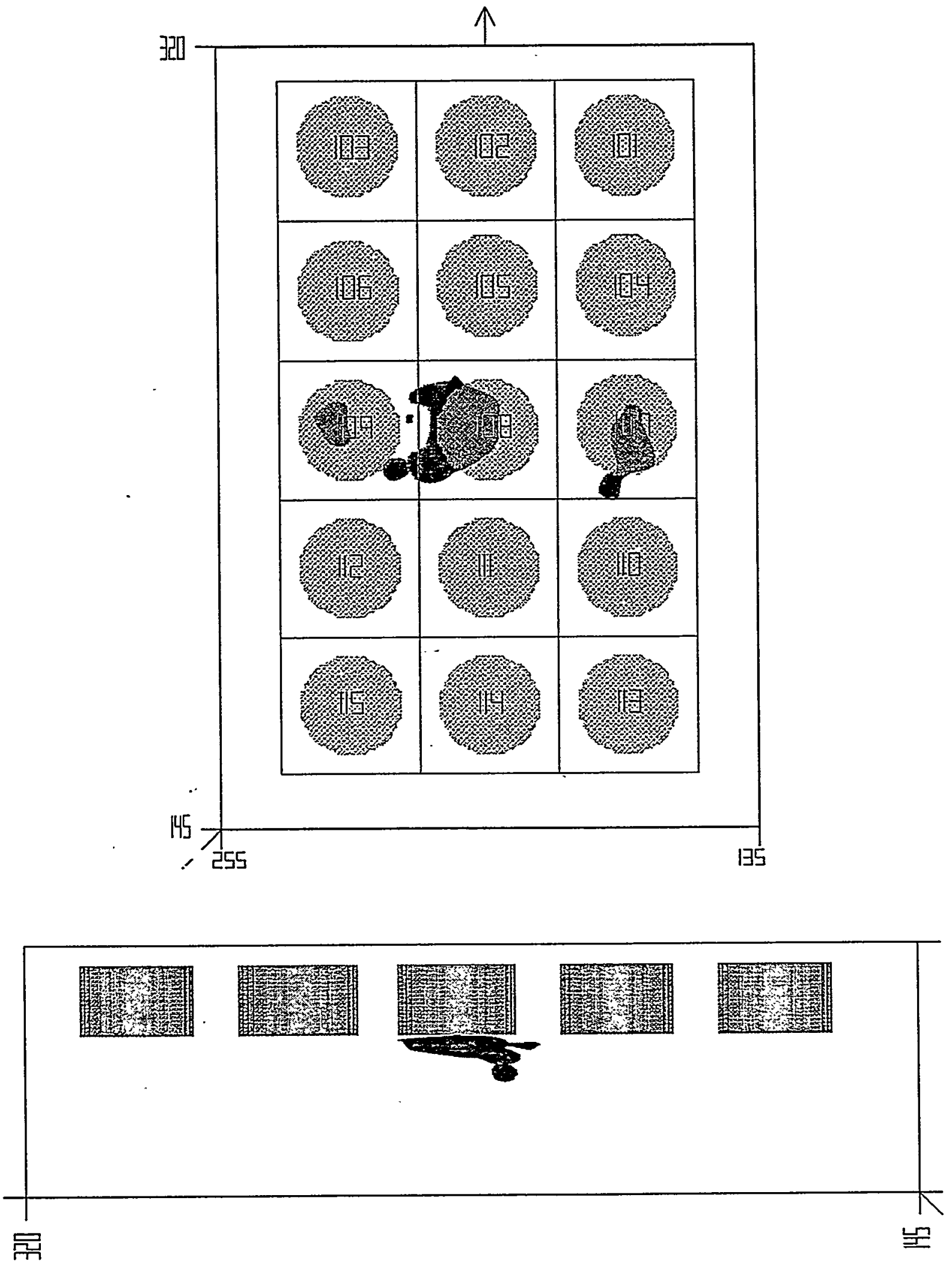

Figure 5. SX kriging point predictions exceeding $5 \times 10^{5} \mathrm{pCi} / \mathrm{g}$ from a top and west view. 

HNF-5782 Rev. 0
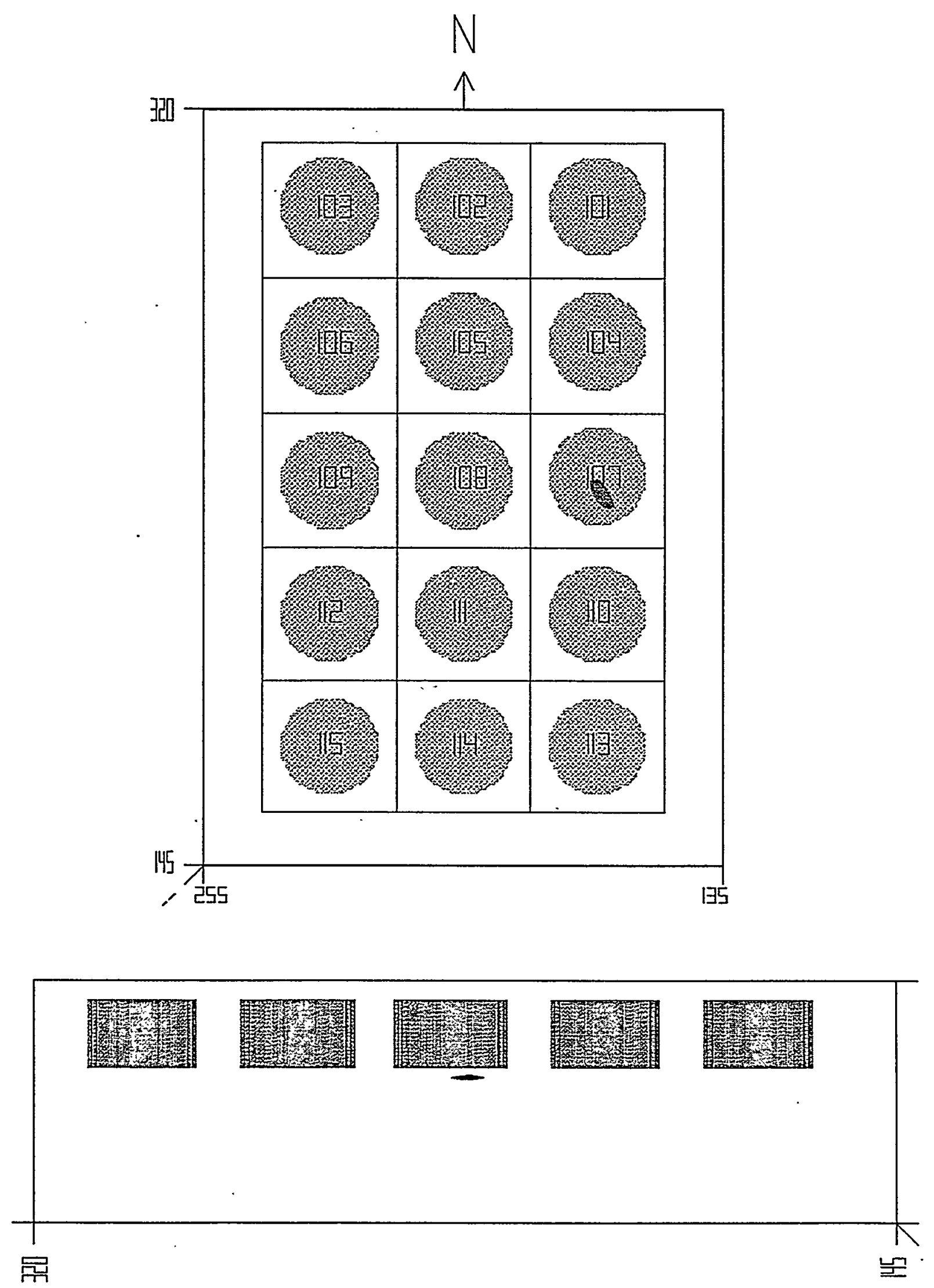

Figure 7. SX kriging point predictions exceeding $5 \times 10^{7} \mathrm{pCi} / \mathrm{g}$ from a top and west view. 


\section{Graphical Locater Home Page}

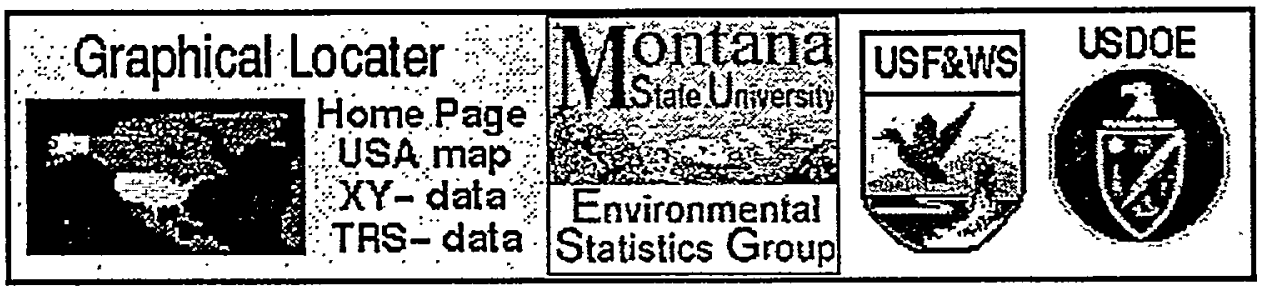

\section{Quick Instructions}

Most users will go straight to the default Graphical Locater starting map (also linked as "USA map" in the lead image), or to one of the other starting maps, and then select an area for a more detailed map. On the more detailed map, you may move to adjacent maps or get information for any location by clicking on it. You may "zoom on that location" to see more detail or use the "Back" button on your browser to return to the map. Please have enough cache available to store to favorite maps. Many users will find it convenient to cut and paste Graphical Locater results into other files. If you need more information come back to this page and read on.

\section{Introduction}

I designed Graphical Locater as an aid for acquiring and refining location data and related measures. It is useful only for people who recognize the position of interest when they see it on the maps. Some users will find it useful to follow along on printed maps that they are more familiar with.

\section{News}

On December 30, I added the HUC maps for regions 1 - 3. This completes the mapping work for the HUC project!, (except for outlying areas).

On December 16, I added a new custom map feature to mark the current location on the HUC map. This may be more useful than the zoom maps for people who known their HUC maps well. Something I hope to encourage! I also modified the distance calculation for nearby named places to not use the UTM projection. I also added a series of state maps with HUC lines on them.

On December 15, I corrected the HUC name lists from NAD27 to NAD83.

On December 14, I modified the interface to Graphical Locater zoom maps so that the distance to the marked point is also reported.

On November 22, I added an new interface to Graphical Locater called TRS-data. This programs allows entry by legal descriptions. The program is based on the trs2ll by Martin Wefald, which covers the western states. I also updated all graphical locater programs for the latest version of trs21l, which now includes North Dakota and Oklahoma.

I am continuing to look for additional data sources, programs and WWW sites for possible addition to the Graphical Locater results. Your suggestions are welcome.

\section{Graphical Locater Starting Maps}


The Graphical Locater starting maps, or level 1 maps, are linked to level 2 maps by an appropriate image configuration file. There are currently 4 starting map areas, the conterminou's U.S.A with many versions, the Greater Montana Area (the original area for Graphical Locater), Puerto Rico and the Virgin Islands, and Hawaii. The conterminous U.S.A maps are based on the area, projection and data of the very useful Conterminous U.S. AVHRR project. I reduced the final image size to 1152 by 725 pixels to fit on at least some screens.

\section{Graphical Locater Level 2 Maps}

The level 2 maps are linked to the Graphical Locater program that returns information for any location. These maps are always pixel-centered maps of straight latitude and longitude. Square pixels are nice, but not required. There are several types of level 2 maps.

- Standard 1:100,000 Maps: These maps cover 1 degree of longitude by 0.5 degree of latitude, exactly the same as the USGS or BLM map series. The title of each map is the official USGS map name, except for Puerto Rico and the Virgin Islands, where Mike Ivie and I made up the names. These maps are all 950 by 620 pixels, which results in a square pixel near the center of the USA (North-South). The panning table at the top of the page allows moving to the next map in any direction. Directions are unlinked when the corresponding map is not available. All areas with 1-degree DEM coverage are included. The line and name data cover only the USA. The limits of the map are at the bottom of the page.

- Conterminous U.S. HUC Regions: These maps were made primarily to support the ongoing project to create Graphical Locater maps for all of the the 8 digit ( 4 code) HUCs. When finished, the HUC link on the Graphical Locater result page will be used to integrate all of these maps and some basic derived information.

- American States: These maps were made for National Wild Fish Health Survey.

- Custom Level 2 Maps: These maps support specific projects. They must be pixel-centered maps of straight latitude and longitude, but size, content and colors may be anything. The referring html file name must start with $\mathrm{xyXYnm}$, where $\mathrm{xy}$ is the southwest corner, $\mathrm{XY}$ is the northeast corner, $\mathrm{n}$ is the number of $x$ pixels and $m$ is the number of $y$ pixels. The format is (2(F7.3,F6.3),2I4) with 0 padding. That should be all you need to make your own custom maps!

- Zoom Maps: These are just custom maps that are made on demand by a Graphical Locater support program. The zoomed point location is shown in the center of the map, in yellow.

\section{Returned Information Details}

1. Latitude/Longitude: The location data in decimal degrees of arc with the degree symbol and hemisphere (North and West). Four decimal digits translates to less than 10 meters. Minutes of arc is 60 multiplied by the fractional part of degrees. Seconds of arc is 60 multiplied by the fractional part of minutes obtained from above.

2. The legal description: This includes, in order, the meridian, township, range, section and state. This program has this information only for the western states. Note that township, range and section define an unique square mile only when the meridian is also given. 
3. UTM: This is the Universal Transverse Mercator zone and $x, y$ coordinates in meters. This conversion is done using the Geodetic Reference System of 1980 (GRS 1980) of the North American Datum of 1983 (NAD 83). Many projects are still using the Clarke Spheroid of 1866 of the NAD 27. Note that a UTM coordinate is meaningless unless the zone is also reported. The UTM coordinates can be used to calculate the distance between to points, as long as the zone is the same.

4. The pixel size: This is given only for entry from a clickable map. The first value is for the E-W direction and the second for the N-S direction to the nearest meter. This is a measure of the expected accuracy. Distances are based on the GRS80 ellipsoid. Non-square pixels are the result of map distortion in the North-South direction. Map distortion does not affect location accuracy, except for the change in pixel size.

5. The distance from the marked point: This is given only for custom maps which already have 1 point defined. Distances are based on the GRS80 ellipsoid and reported to the nearest meter.

6. The elevation: The height above mean sea level in meters and feet. This is based on simple bilinear interpolation of the original 1-degree ( 3 arc second) digital elevation model (DEM) data.

7. The gradient: This is the maximum slope through the point. Slope is feature of a line, not a point. The gradient is calculated from the $\mathrm{X}$-direction slope and $\mathrm{Y}$-direction slope. The directional slopes are based on bilinear interpolation of elevation over a radius of 35 meters. The reported gradient is thus a very local measure (about 1 pixel on the standard maps). Any location within 1 arc second of a whole degree cell edge is moved slightly inward to prevent the need to bridge across the DEM units and the discrepancies that occur there.

8. The aspect direction: This is the direction of downhill gradient. I am using 0 for North, 90 for East, 180 for South and 270 for West. The standard abbreviation of the 8 compass directions is given after the number value. Note that this is a circular statistic with 359 and 1 being almost the same, true North. The aspect calculation is based on the X-direction and $\mathrm{Y}$-direction slopes. The directional slopes are based on bilinear interpolation of elevation over a radius of 35 meters. The reported aspect is thus a very local measure. Any location within 1 arc second of a whole degree cell edge is moved slightly inward to prevent the need to bridge across the DEM units and the discrepancies that occur there.

9. The local roughness: This is simply the standard deviation of surrounding elevation values. This calculation uses the nearest 16 elevation values in the original 1-degree ( 3 arc second) digital elevation model (DEM) data. This corresponds to a radius of about 125 meters. Multiple 1-degree units are not never combined for this calculation to avoid the discrepancies that occur there. Variation in elevation is only 1 of 3 components of terrain roughness. Variation in gradient and variation in aspect are too demanding to calculate here. The local roughness interpretation is based on almost 100,000 equally spaced points across the USA. Flat is for no variation in elevation; slight is above zero and below the 25 th percentile, average is between the 25 th and 75 th percentiles, moderate is between the 75 th and 95 th percentiles and extreme is over the 95 th percentile.

10. The location $(X, Y ; Z)$ : This is my choice for standard data entry, many others are possible. West longitudes are negative and $X$ (longitude) comes before $Y$ (latitude).

11. The state, county and FIPS code: This determination is based on a $850 \mathrm{~m}$ raster grid created from 1:2,000,000 DLG data. It will be a little "rough on the edges". A test run against the GNIS gave the 
error rate at $3.16 \%$, but some of the errors are in the GNIS. Many of the errors are named peaks that occur on the county line.

12. The hydrologic unit name and code: These are the USGS 8-digit HUC composed of 4 2-digit codes that give the region, subregion, accounting unit and catalog unit. The "Place point in HUC" link allows you to mark the current position in the current HUC map. The HUC determination is based on a $850 \mathrm{~m}$ raster grid created from the $1: 2,000,000 \mathrm{DLG}$ data that were further refined in our lab. Our refined version should match the USGS intent, except for California subregion 2 accounting unit 1 and subregions 3 and 4 . In these areas, some units were constructed so poorly that they have no utility for our purposes and they were restructured to better match drainage borders. The HUC is linked to our own HUC summary page that features 3 graphical locater maps for each unit. Because of the grid size, the HUC determination will be a little "rough at the edges". A test run of almost 1000 sample sites in one of my projects, gave the error rate at $0.85 \%$. It also discovered one error in my data! Most errors occur at the edges of the units (mouth or headwaters) and these can be easily double checked by selecting a more central location.

13. The Omernik ecoregion name and number: These are the 76 regions used by Omernik, 1987 for the conterminous United States. The more or less typical flag is also given. The ecoregion determination is based on a $850^{\circ} \mathrm{m}$ grid and will be a little "rough at the edges". The error rate should be similar to that of the HUC and FIPS determination, but I have no independent data to check.

14. The 1:100,000 map: This is a link to the GL 1:100,000 map without checking to see if that map exists.

15. Zoom on that location: This is a link to the GL map making program at will make a large scale (small area) map centered on the selected location. If there are no data within that area, you will get a blank map.

16. Try TerraServer: This is a link to Microsoft's TerraServèr centered on the selected location, but without any way to known if an image exists for that location.

17. Nearby named places: These are from the Geographic Names Information System (GNIS). Linear features are split into "mouth" and "head" points. The state and county names replace the original FIPS codes, except for "head" locations. Here, the state and county might be wrong, so they are left out. I converted the position data (X,Y as decimal degrees of arc) from NAD 27 to NAD 83 to be more comparable with the TIGER/LINE 1995 line data. The elevation, if given, is the original GNIS data in feet. The distance from the selected location is based on the GRS80 ellipsoid. Selecting the position of a named place makes it the current GL position and allows zooming on that position exactly (or within a few meters due to rounding).

18. The 7.5 minute series topographic maps for that area: This is based on the map names part of the Geographic Names Information System (GNIS). The map names are in a 3 by 3 table similar the "adjoining map table" on many printed maps. The top is North and right is East. The selected point is on the map that is named within the middle cell.

\section{Accuracy}

The positional accuracy of Graphical Locater is limited by the pixel size (reported with every location), the users aim (unknown), and the underlying map data ( $+/$ - about 50 meters for the 1:100,000 map data). The program introduces no error of its own, as far as I know. Graphical Locater results are better than any other 
method available to me, so I have no way to independently test its accuracy, others are invited to do so and report back.

The median absolute difference between Graphical Locater elevations and the elevation reported for over 30,000 named places in the us_concise file of the GNIS was 5.5 meters. Of the 10 largest errors, all were in the name file. As with all grid interpolations, the elevation of peaks will be low and the elevations of dips will be high. The roughness gives a measure of the point elevation uncertainty.

\section{Base Data}

- Geographic Names Information System This is described also by the GLIS GNIS page. I obtained a copy of all units, except Antarctica in September 1997. I discarded all records without location data as well as records for springs and wells that lacked real names. I split linear features into "mouth" and "head" records. "Head" records lack state and county. Some linear feature mouths have the state and county of the head location!

- Small scale (1-degree) Digital Elevation Models. I obtained a complete set of 957 files from the USGS FTP site in September 1997. These are used to generate the shaded relief in all level 2 Graphical Locater maps. They are used by the Graphical Locater program to interpolate the elevation and related measures for any location.

- TIGER/Line '95 I obtained a complete copy of these files from the MSU Library on 6 CD-ROMs. These data are based on the USGS 1:100,000-scale Digital Line Graph Data, but are more convenient for big projects. These are used to map the roads, water-bodies, streams and canals on all large-scale Graphical Locater maps.

\section{Base Programs}

\section{Acknowledgments}

The U.S. Geological Survey deserves much credit for making massive amounts of useful mapping data and programs freely available. Martin Wefald very kindly made his most useful trs2ll program available to handle the township-range-section conversions. Mike Ivie, here at MSU-Bozeman has offered many suggestions on this project. Much of the upgrades to Graphical Locater were funded by the U.S. Fish and Wildlife Service to help support the National Wild Fish Health Survey.

30 OCT 1995, last updated on 16 DEC 1999

D.L.Gustafson, dlg@rapid.msu.montana.edu 


\section{Graphical Locater Starting Maps}

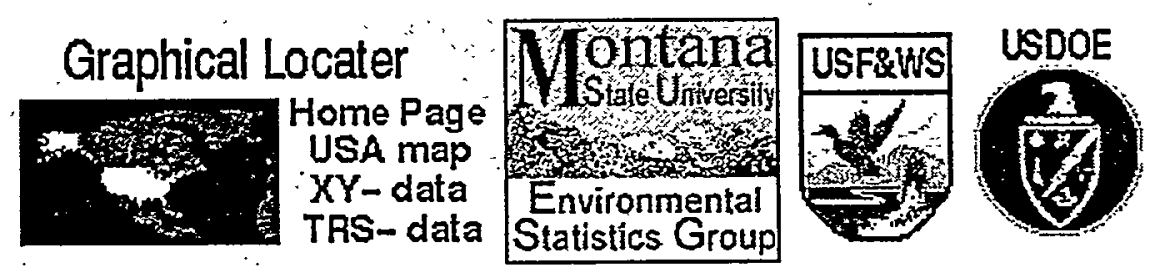

This page links to all of the first level graphical locater maps. Level one maps maps lead to level two maps. The level two maps lead to the Graphical Locater program, which returns information for any location.

\section{Conterminous U.S.A Maps}

\begin{tabular}{|c|c|c|}
\hline Relief with elevation & NDVI with relief & AVHRR with relief \\
\hline  &  & 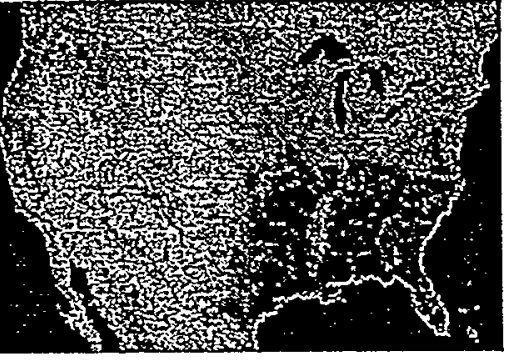 \\
\hline Major rivers only & Major rivers only & Major rivers only \\
\hline Rivers and lakes & Rivers and lakes & Rivers and lakes \\
\hline Hydrologic units with cells & Hydrologic units with cells & Hydrologic units with cells \\
\hline Rivers and states with cells* & Rivers and states with cells & Rivers and states with cells \\
\hline States and counties with cells & States and counties with cells & States and counties with cel: \\
\hline Omernik ecoregions with cells & Omernik ecoregions with cells & Omernik ecoregions with cel \\
\hline
\end{tabular}

* The default starting map.

\section{Other Level 1 Maps}

- Greater Montana Area

- Puerto Rico and the Virgin Islands

- Hawaii

\section{Large Level 2 Maps}


- Conterminous U.S. and the 50 Individual States

- Conterminous U.S. HUC Regions

26 NOV 1997, last updated on 2 DEC 1999 D.L. Gustafson

dlg@rapid.msu.montana.edu 


\section{TRS-data}

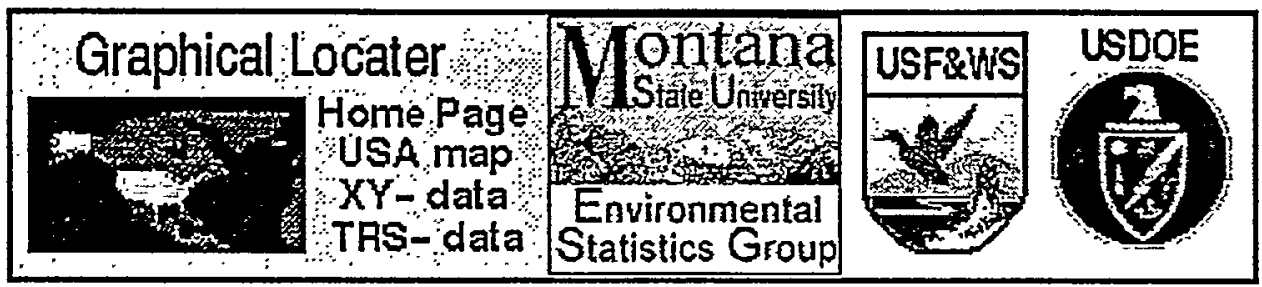

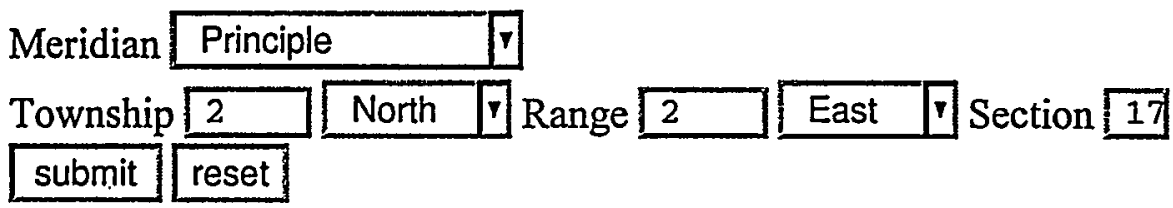

Instructions: This program returns the same information as Graphical Locater for a valid legal description within the western states (AZ, CA, CO, ID, KS, MT, ND, NE, NM, NV, OK, OR, SD, UT, WA and WY). The returned position is for the center of the section. Select the Meridian and directions and input the Township, Range, and Section numbers and select submit. If you do not known your Meridian, use the Graphical Locater USA map and select a position near your legal description. The meridian is given as the first part of the legal description (line 2). If the legal description is "unknown" to Graphical Locater, this program will not work either. Also be aware that some meridians are quite small, so some trial and error may be necessary. The California BLM has a nice set of maps showing the Principal Meridians and Base Lines and the areas they cover.

Introduction: The United States Public Land Survey (USPLS) started with the Land Ordinance of 1785 and covers all US land that was not settled by the time of the official government survey. It does not include the east coast states including Kentucky and Tennessee or Texas. Small areas of other states that were settled before the survey also technically excluded. This explains the odd shapes of many mining claims in the west. Latter title transfers followed USPLS lines.

Legal descriptions to 1 mile square sections have 4 parts: the Meridian, Range, Township and section number. Note that an official legal description always works from small to large areas, for example NW1/4, sec. 12, T.122N, R.71W, Fifth Principle Meridian.

- Meridian These are similar to UTM zones. Both apply to large areas based on a line of longitude, both are often left out of field data, and the position is not uniquely defined without them! The position of the principal meridian for any area, in contrast to UTM zones, is largely historical and arbitrary. The area governed by each Meridian, also in contrast to UTM zones, is irregular in size and shape, but it frequently follows state lines. Actually it is more correct to say that the states were formed according to the areas covered by the Meridians. Some of these areas include several large states while others include only a small part of a single state. Some of the smaller areas were caused by changes in tribal reservations. The default principal meridian for this program (Principle) is good for (as you might guess) Montana.

- Township These occur at 6 mile intervals east and west of the principal meridian. Township values are normally whole numbers starting at 1 , but some exceptions occur. Township lines are normally adjusted every 4th line to correct for the spherical nature of the earth. These are called standard parallels. 
- Range These occur at 6 mile intervals north and south of a base line that is associated with each principal meridian. The position of the base line for each principal meridian is also historical and arbitrary. Range values are normally whole numbers starting at 1, but some exceptions occur. Range lines are normally adjusted every 4 th line to correct for the spherical nature of the earth. These are called guide meridians.

- Sections The intersection of Range lines and Township lines define 6 by 6 mile squares called Townships, which are divided into 36 sections. These are normally 1 by 1 mile squares, but some are altered to correct for the spherical earth. Most of these departures are pushed to the northern and western tiers of sections before the next standard parallels and guide meridians. Others are altered by simple survey errors. Note that old survey errors have legal precedence over new survey results so the odd-looking lines stay put. Section numbers always range between 1 and 36. Sections are normally divided by repeated quartering, but this is beyond the scope of this program.

The USPLS serves perfectly well for its intended used of land title management, but it is not very suitable for scientific point location management. Mapping and spatial statistics are much easier with a system that is more regular over large areas. The conversion to latitude/longitude allows the USPLS data to be better used in many other systems. The key conversion code is the trs2ll program by Martin Wefald, who has kindly made his program available for this project. The Graphical Locater interface allows easy verification and fine tuning of the position data.

NOV 16 1999, updated on 22 NOV 1999

Web interface and Graphical Locater by D.L. Gustafson, dlg@rapid.msu.montana.edu The trs2ll program by Martin Wefald, wefald@crl.com. 


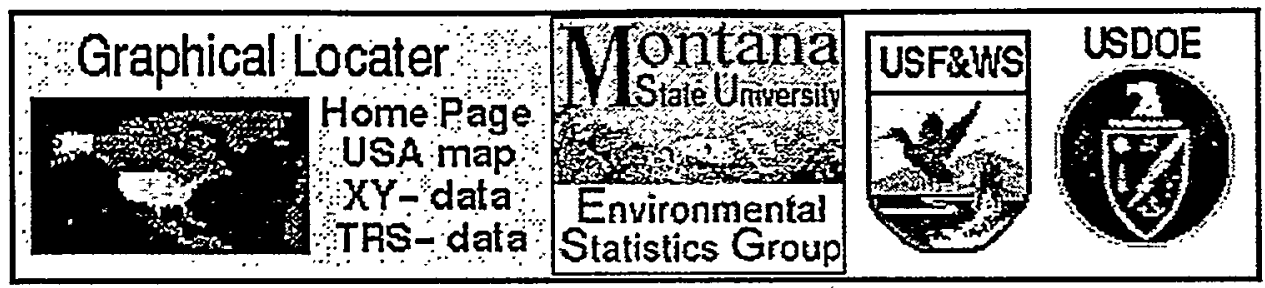

\section{The selected location is:}

Latitude/Longitude $45.9261^{\circ} \mathrm{N}, 111.5030^{\circ} \mathrm{W}$

The legal description is Principle T2N,R2E,sec17 MT

UTM zone $12(\mathrm{X}, \mathrm{Y})$ 461000, 5085962

The elevation is $1224 \mathrm{~m}(4016 \mathrm{ft})$

The gradient is: 0.0 percent

There is no aspect direction.

The local roughness is: 0.0 or flat

The location as decimal degrees $(\mathrm{X}, \mathrm{Y} ; \mathrm{Z})=-111.5030,45.9261 ; 1224 \mathrm{~m}$

The state and county are Montana: Gallatin County 30031

The HUC is Madison 10020007; Place point in HUC

The Omernik ecoregion is Montana Valley and Foothill Prairies (more typical) 16

The 1:100,000 map (if available); Try TerraServer

Zoom on that location with radius $=\underline{2 \mathrm{~km}} ; \underline{5 \mathrm{~km}} ; \underline{10 \mathrm{~km}} ; \underline{20 \mathrm{~km}} ; \underline{30 \mathrm{~km}}$.

\section{Nearby named places (in order by distance)}

1. Headwaters Cemetery; Montana: Gallatin Co. $-111.4994,45.9277$ at a distance of $331 \mathrm{~m}$

2. Madison River; Montana: Gallatin Co. $-111.5077,45.9268$ at a distance of $373 \mathrm{~m}$

3. Missouri River head; $-111.5080,45.9274$ at a distance of $415 \mathrm{~m}$

4. Missouri River head; $-111.5080,45.9274$ at a distance of $415 \mathrm{~m}$

5. Missouri River mouth; Montana: Roosevelt Co. $-111.5080,45.9274$ at a distance of $415 \mathrm{~m}$

6. Jefferson River mouth; Montana: Gallatin Co. $-111.5080,45.9274$ at a distance of $415 \mathrm{~m}$

7. Missouri River head; $-111.5080,45.9274$ at a distance of $415 \mathrm{~m}$

8. Gallatin (historical); Montana: Gallatin Co. $-111.4980,45.9277,(4050 \mathrm{ft}$ ) at a distance of $426 \mathrm{~m}$

9. Gallatin (historical); Montana: Gallatin Co. $-111.4980,45.9277$ at a distance of $426 \mathrm{~m}$

10. Gallatin Post Office (historical); Montana: Gallatin Co. $\underline{-111.4980,45.9277}$ at a distance of $426 \mathrm{~m}$

\section{The 7.5 minute series topographic maps for that area}




\begin{tabular}{|c|c|c|}
\hline $\begin{array}{l}\text { Radersburg } \\
\text { SW }\end{array}$ & Plunket Lake & Lombard \\
\hline $\begin{array}{l}\text { Milligan } \\
\text { Canyon }\end{array}$ & Three Forks & Logan \\
\hline Willow Creek & $\begin{array}{l}\text { Three Forks } \\
\text { SE }\end{array}$ & Manhattan SW \\
\hline
\end{tabular}

This was request number 123940

dlg@rapid.msu.montana.edu 


\section{Bozeman}

\begin{tabular}{|c|c|c|}
\hline$\underline{N W}$ & $\underline{N}$ & $\underline{N E}$ \\
\hline$\underline{W}$ & & $\underline{E}$ \\
\hline$\underline{S W}$ & $\underline{S}$ & $\underline{S E}$ \\
\hline
\end{tabular}

Use the table above to move one map in any direction, or select a location on the map below.



West $=-112.0$, South $=45.5$, East $=-111.0$, North $=46.0$

11 NOV 1997, last updated on 10 MAR 1998 D.L. Gustafson, dlg@rivers.oscs.montana.edu

Back to Graphical Locater. 


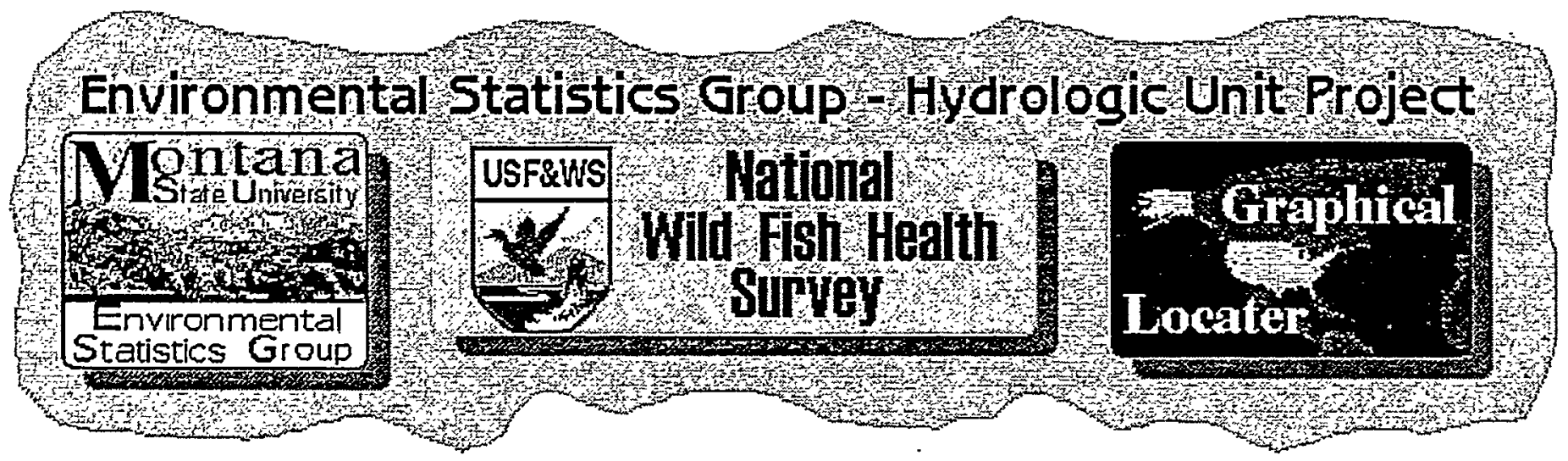

\begin{tabular}{|l|c|}
\hline$\not \lambda$. & UNDER \\
\hline CONSTRUCTION \\
\hline
\end{tabular}

\section{Madison -- Cataloging Unit 10020007}

This HUC is part of the:

- Missouri Headwaters Subregion 1002

- Missouri Region 10

\section{Graphical Locater Maps}

Roads Water Only 8 Digit HUCs

The area of this HUC in these maps is $6634.7 \mathrm{~km}^{2}\left(2562.8 \mathrm{mi}^{2}\right)$.

The area from the USGS is $2570 \mathrm{mi}^{2}$.

\section{Named places in this watershed 1581}

\section{Elevation Analysis}

This analysis is based on 2649899 elevations on a $50 \mathrm{~m}$ grid within the area of this HUC.

The mean elevation is $2156.9 \mathrm{~m}$ ( $7076.4 \mathrm{ft})$.

The elevation standard deviation is $401.9 \mathrm{~m}$ ( $1318.6 \mathrm{ft}$ ).

The coefficient of variation is $18.6 \%$.

The vertical relief is $2180.2 \mathrm{~m}(7152.8 \mathrm{ft})$.

Elevation Percentiles

\begin{tabular}{|c|c|c|c|c|c|c|c|c|c|c|c|}
\hline & $0 \%$ & $10 \%$ & $20 \%$ & $30 \%$ & $40 \%$ & $50 \%$ & $60 \%$ & $70 \%$ & $80 \%$ & $90 \%$ & $100 \%$ \\
\hline meters & 1224.0 & 1584.0 & 1743.4 & 1965.7 & 2072.0 & 2201.0 & 2316.0 & 2415.9 & 2501.0 & 2630.4 & 3404.2 \\
\hline feet & 4015.7 & 5196.8 & 5719.7 & 6449.1 & 6797.8 & 7221.0 & 7598.3 & 7926.1 & 8205.3 & 8629.8 & 11168.5 \\
\hline
\end{tabular}




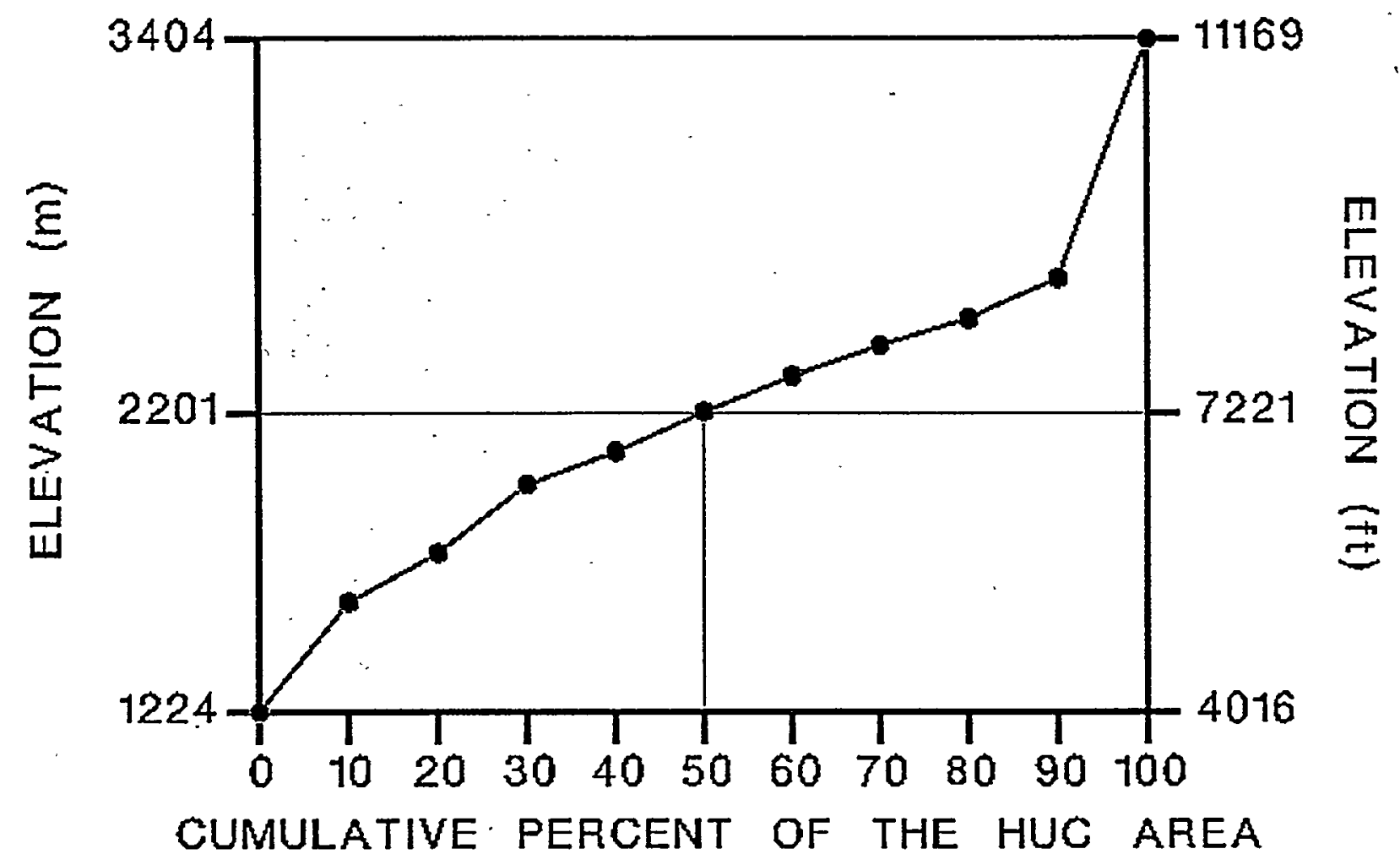

\section{Map Line Analysis}

\begin{tabular}{|l|l|l|l|l|}
\hline line type & $\mathbf{k m}$ & $\mathbf{m i}$ & $\mathbf{k m} / \mathbf{k m} \mathbf{2}^{2}$ & $\mathbf{\mathbf { m i }} / \mathbf{m i}^{\mathbf{2}}$ \\
\hline \hline Perenial Shoreline & 622.4 & 386.8 & 0.094 & 0.151 \\
\hline \hline Intermittent Shoreline & 8.3 & 5.2 & 0.001 & 0.002 \\
\hline \hline Perenial Streams & 2785.8 & 1731.4 & 0.420 & 0.676 \\
\hline \hline Intermittent Streams & 1802.2 & 1120.0 & 0.272 & 0.437 \\
\hline Canals & 439.2 & 273.0 & 0.066 & 0.107 \\
\hline \hline $1^{\circ}$ roads & 5.2 & 3.2 & 0.001 & 0.001 \\
\hline \hline $2^{\circ}$ roads & 245.3 & 152.5 & 0.037 & 0.059 \\
\hline $3^{\circ}$ roads & 65.4 & 40.7 & 0.010 & 0.016 \\
\hline $4^{\circ}$ roads & 2361.6 & 1467.7 & 0.356 & 0.573 \\
\hline Railroads & 29.8 & 18.5 & 0.004 & 0.007 \\
\hline \hline Pipes and Powerlines & 111.8 & 69.5 & 0.017 & 0.027 \\
\hline \hline
\end{tabular}

EPA-Surf Your Watershed/ National Watershed Characterization 
USGS-Science in Your Watershed/ Selected Realtime Streamflow Stations

4 SEPT 1999, Last updated on 30-Dec-99

D.L. Gustafson, dlg@,rapid.msu.montana.edu

The Graphical Locater Home Page. 


\section{Madison 10020007}

Select a location.

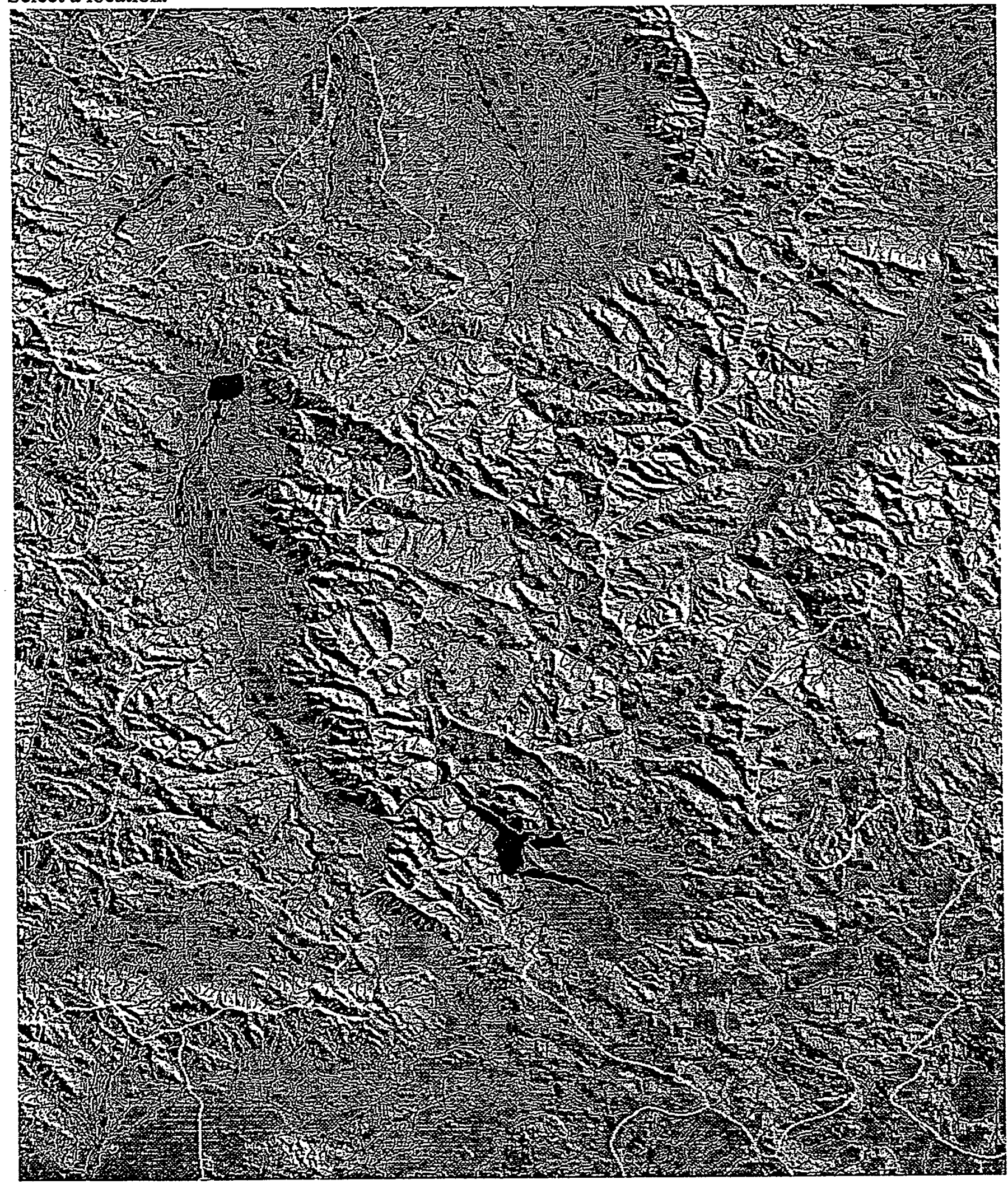




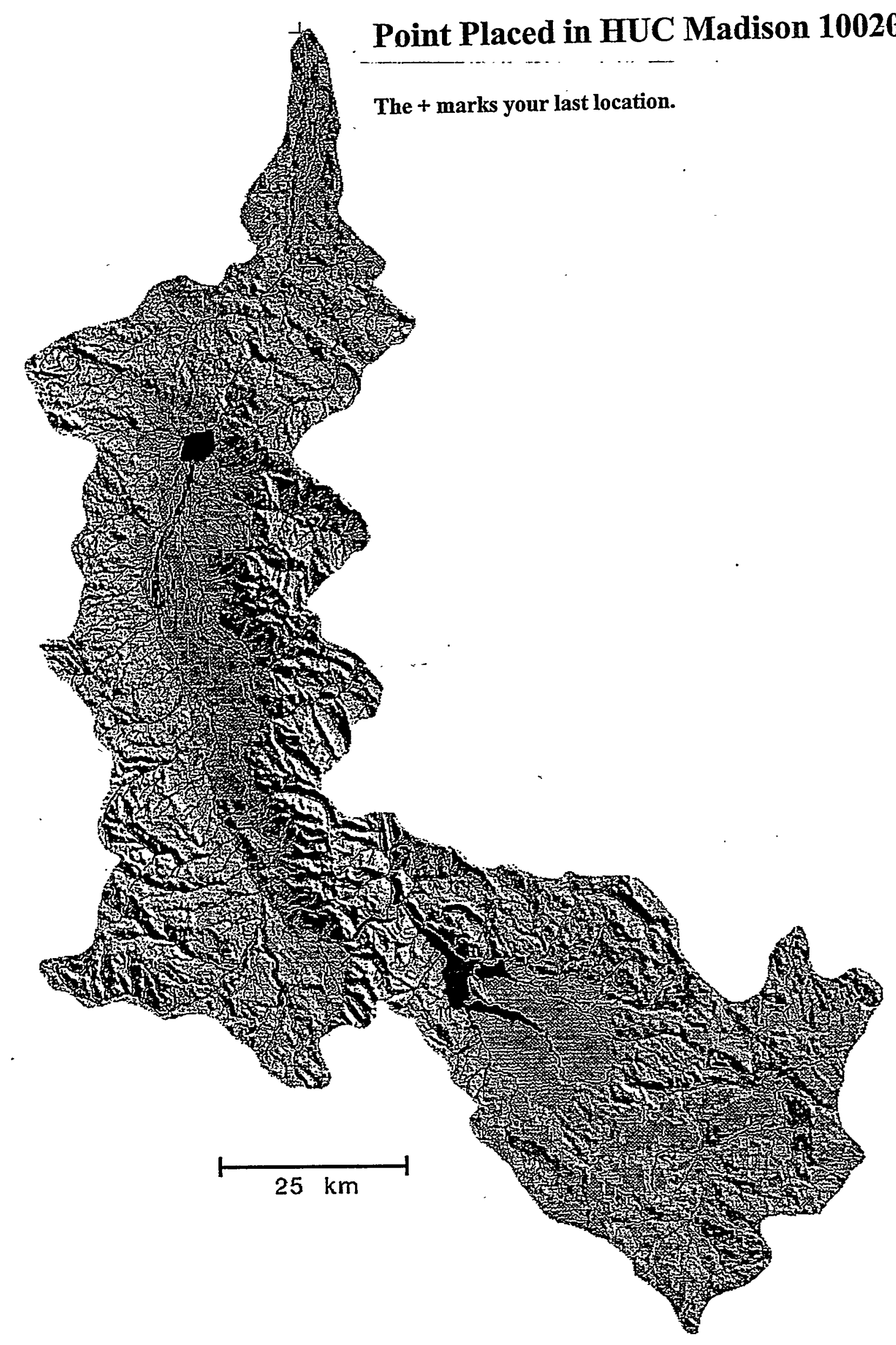




\section{Missouri Headwaters 1002}






\section{Missouri Region 10}






60. Mosquito Gulch mouth

61. ? Norwegian Placer Gulch mouth

62. Pony Gulch head

63. Pony Gulch mouth

64. Red Canyon Fault head

65. Red Canyon Fault mouth

66. Rocky Draw head

67. Rocky Draw mouth

68. Secret Valley head

69. Secret Valley mouth

70. Sunrise Gulch head

71. Sunrise Gulch mouth

72. Wall Canyon head

73. Wall Canyon mouth

74. Well Gulch head

75. Well Gulch mouth

- well

1. ? Mud Pot

11 SEPT 1999, last updated on 15-Dec-99 D.L.Gustafson, dlg@rapid.msu.montana.edu Back to Madison 


\section{Graphical Locater Level 2 Maps}

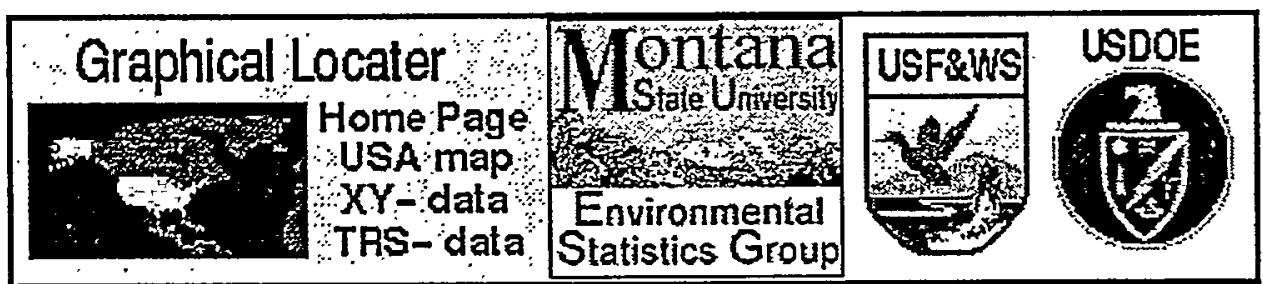

\section{Large area Graphical Locater Level 2 Maps}

- Northwest Quarter of the Earth

- Conterminous USA

- USA States

- Conterminous USA HUC Regions

\section{Graphical Locater Custom Level 2 Maps}

Gulf of Mexico and Caribbean Sea

West Indies

Columbia River Drainage

Greater Montana Area HUC Coverage

Henry's Fork River Drainage, also available without roads

Greater Yellowstone Area, also available without roads

National Wildlife Refuges in the Intermountain West

- Benton Lake National Wildlife Refuge

- Grays Lake National Wildlife Refuge

- Red Rock Lakes National Wildlife Refuge

U.S. Virgin Islands

- Saint Thomas

- Saint John

- Saint Croix

\section{Canyon Ferry Lake}

You may now make your own custom level 2 maps with Graphical Locater Custom Mapper

29 NOV 1997, last updated on 2 DEC 1999, D.L. Gustafson, dlg@rapid.msu.montana.edu 


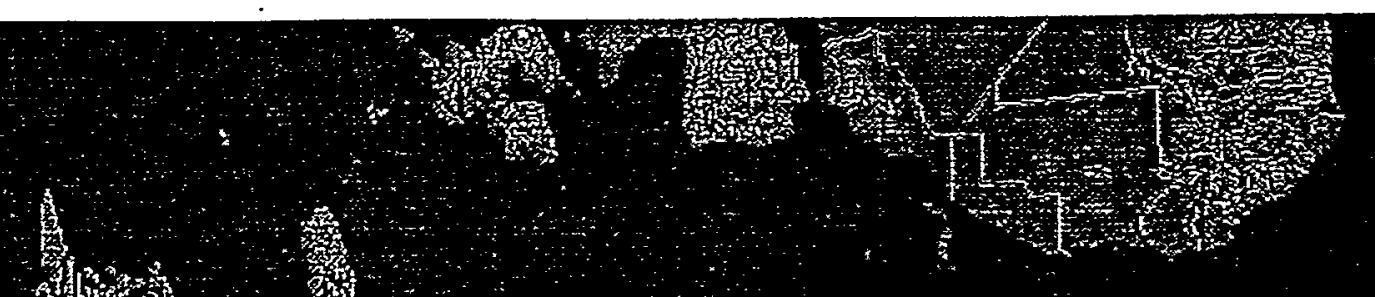

36)



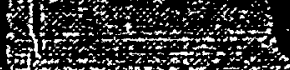

5

16

7.

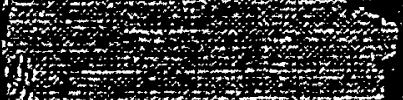

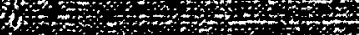

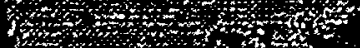

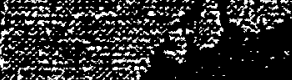




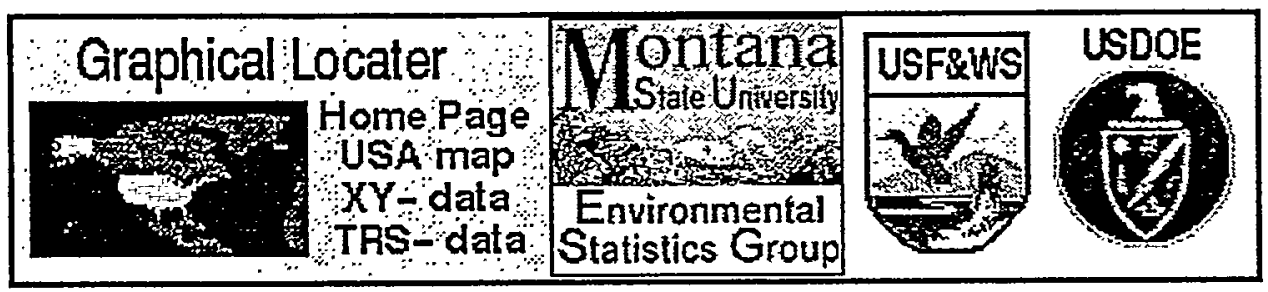

\section{Conterminous USA Maps}

1. Roads 1

2. Roads 2

3. Roads 3

4. Water only

5. HUC regions

6. HUC regions and subregions

7. HUC regions, subregions and cataloging units

8. HUC cells

9. State lines

24 FEB 1999, last updated on 06 JAN 2000

D.L.Gustafson, dlg@rapid.msu.montana.edu 


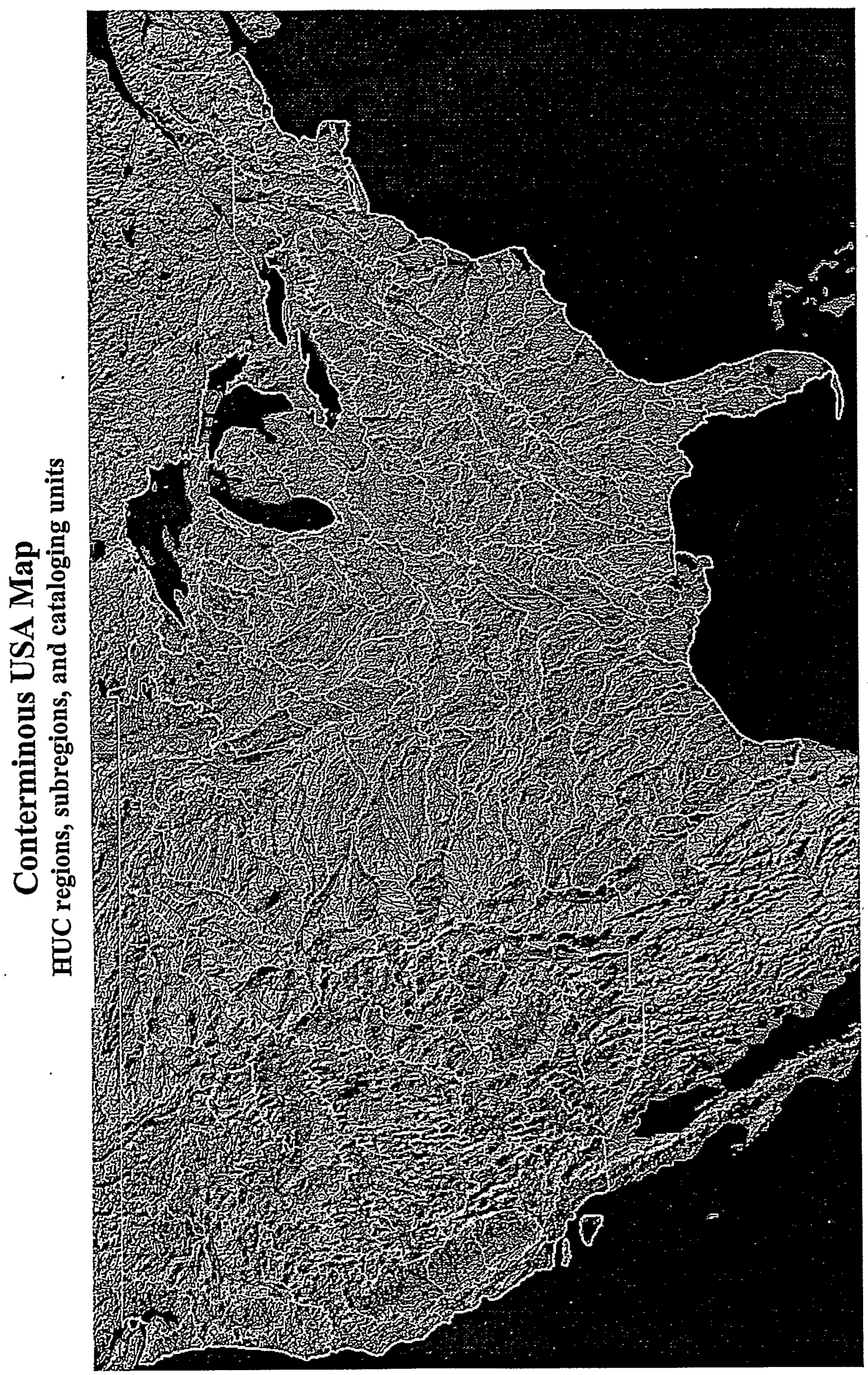




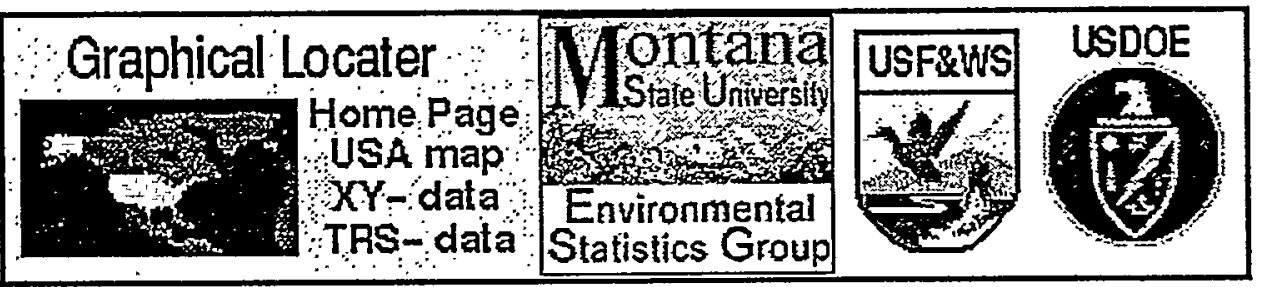

\section{USA State Maps}

\begin{tabular}{|c|c|c|c|c|}
\hline Alabama & major roads & water only & HUC lines & $\underline{\text { HUC cells }}$ \\
\hline Alaska & major roads & water only & -- & - \\
\hline Arizona & major roads & water only & HUC lines & HUC cells \\
\hline Arkansas & major roads & water only & HUC lines & HUC cells \\
\hline California & major roads & water only & HUC lines & $\underline{\text { HUC cells }}$ \\
\hline Colorado & major roads & water only & FUC lines & $\underline{\text { HUC cells }}$ \\
\hline Connecticut & major roads & water only & HUC lines & HUC cells \\
\hline Delaware & major roads & water only & HUC lines & HUC cells \\
\hline Florida & major roads & water only & HUC lines & HUC cells \\
\hline Georgia & major roads & water only & $\underline{\text { HUC lines }}$ & HUC cells \\
\hline Hawaii & major roads & water only & -- & $-\cdots$ \\
\hline Idaho & major roads & water only & HUC lines & HUC cells \\
\hline Illinois & major roads & water only & HUC lines & HUC cells \\
\hline Indiana & major roads & water only & HUC lines & HUC cells \\
\hline Iowa & major roads & water only & HUC lines & HUC cells \\
\hline Kansas & major roads & water only & HUC lines & HUC cells \\
\hline Kentucky & major roads & water only & HUC lines & HUC cells \\
\hline Louisiana & major roads & water only & HUC lines & HUC cells \\
\hline Maine & major roads & water only & HUC lines & HUC cells \\
\hline Maryland & major roads & water only & $\underline{\text { HUC lines }}$ & $\underline{\mathrm{HUC} \text { cells }}$ \\
\hline Massachusetts & major roads & water only & HuC lines & HUC cells \\
\hline Michigan & major roads & water only & $\underline{\mathrm{HU}}$ & $\underline{\mathrm{HU}}$ \\
\hline Minnesota & major roads & water only & HUC lines. & $\underline{\mathrm{HU}}$ \\
\hline
\end{tabular}




\begin{tabular}{|c|c|c|c|c|}
\hline Mississippi & major roads & water only & HUC lines & HUC cells \\
\hline Missouri & major roads & water only & HUC lines & $\underline{\text { HUC cells }}$ \\
\hline Montana & major roads & water only & HUC lines & $\underline{\text { HUC cells }}$ \\
\hline Nebraska & major roads & water only & HUC lines & HUC cells \\
\hline Nevada & major roads & water only & HUC lines & HUC cells \\
\hline New Hampshire & major roads & water only & HUC lines & HUC cells \\
\hline New Jersey & major roads & water only & HUC lines & HUC cells \\
\hline New Mexico & major roads & water only & HUC lines & HUC cells \\
\hline New York & major roads & water only & HUC lines & HUC cells \\
\hline North Carolina & major roads & water only & $\underline{\text { HUC lines }}$ & $\underline{\mathrm{HUC}}$ cells \\
\hline North Dakota & major roads & water only & HUC lines & HUC cells \\
\hline Ohio & major roads & water only & lines & HUC cells \\
\hline Oklahoma & major roads & water only & lines & HUC cells \\
\hline Oregon & major roads & water only & lines & HUC cells \\
\hline Pennsylvania & major roads & water only & HUC lines & HUC cells \\
\hline Rhode Island & major roads & water only & HUC lines & HUC cells \\
\hline South Carolina & major roads & water only & HUC lines & $\underline{\text { HUC cells }}$ \\
\hline South Dakota & major roads & water only & HUC lines & HUC cells \\
\hline Tennessee & major roads & water only & HUC lines & $\underline{\mathrm{HUC} \text { cells }}$ \\
\hline Texas & major roads & water only & HUC lines & $\underline{\text { HUC cells }}$ \\
\hline Utah & major roads & water only & HUC lines & HUC cells \\
\hline Vermont & major roads & water only & HUC lines & HUC cells \\
\hline Virginia & major roads & water only & HUUC lines & $\underline{\text { HUC cells }}$ \\
\hline \begin{tabular}{|l} 
Washington \\
\end{tabular} & major roads & water only & lines & $\underline{\text { HUC cells }}$ \\
\hline West Virginia & major roads & water only & HUC lines & HUC cells \\
\hline Wisconsin & major roads & water only & HUC lines & $\underline{\text { HUC cells }}$ \\
\hline Wyoming & major roads & water only & HUC lines & HUC cells \\
\hline
\end{tabular}

1 APR 1999, last updated on 17 DEC 1999 D.L. Gustafson 


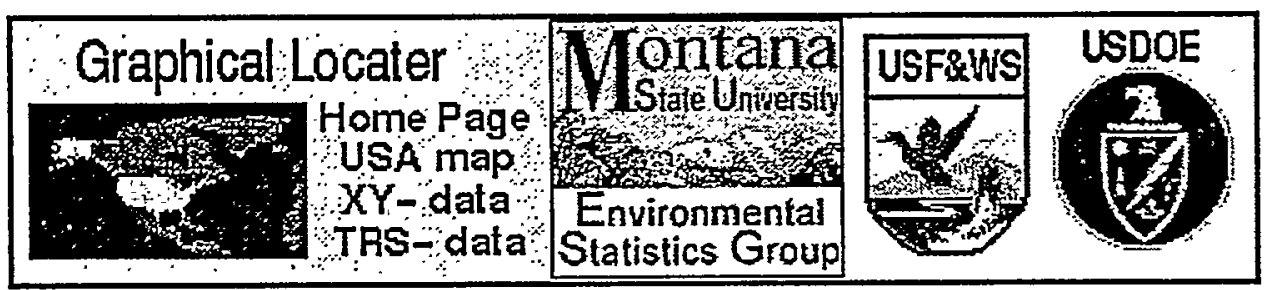

\section{HUC Region Maps}

\begin{tabular}{|c|c|c|c|c|c|}
\hline 01 & New England Region & major roads & water only & HUC lines & HUC cells \\
\hline 02 & Mid-Atlantic Region & major roads & water only & HUC lines & HUC cells \\
\hline 03 & South Atlantic-Gulf Region & major roads & water only & HUC lines & HUC cells \\
\hline 04 & Great Lakes Region & major roads & water only & HUC lines & HUC cells \\
\hline 05 & Ohio Region & major roads & water only & HUC lines & HUC cells \\
\hline 06 & Tennessee Region & major roads & water only & HUC lines & HUC cells \\
\hline 07 & Upper Mississippi Region & major roads & water only & HUC lines & $\underline{\text { HUC cells }}$ \\
\hline 08 & Lower Mississippi Region & major roads & water only & HUC lines & HUC cells \\
\hline 09 & Souris-Red-Rainy Region & major roads & water only & HUC lines & HUC cells \\
\hline 10 & Missouri Region & major roads & water only & HUC lines & HUC cells \\
\hline 11 & Arkansas-White-Red Region & major roads & water only & lines & ells \\
\hline 12 & Texas-Gulf Region & major roads & water only & ines & ells \\
\hline 13 & Rio Grande Region & major roads & wat & $\underline{\text { HUC }}$ & HUC cells \\
\hline 14 & Upper Colorado Region & major roads & water only & HUC lines & HUC cells \\
\hline 15 & Lower Colorado Region & major roads & water only & HUC lines & HUC cells \\
\hline 16 & Great Basin Region & major roads & water only & lines & C cells \\
\hline 17 & Pacific Northwest Region & major roads & water only & HUC lines & cells \\
\hline 18 & California Region & major roads & water only & HUC lines & HUC cells \\
\hline
\end{tabular}

HUC progress report

12 JAN 1998, last updated on 17 DEC 1999 D.L. Gustafson

dlg@rapid.msu.montana.edu 
